Supporting Information for

\title{
Asymmetric Synthesis of
}

\section{Monofluorinated 1-Amino-1,2- dihydronaphthalene and 1,3-}

\section{Aminoalcohol Derivatives.}

\author{
Rubén Lázaro, ${ }^{\mathrm{a}, \mathrm{b}}$ Raquel Román, ${ }^{\mathrm{a}}$ Daniel M. Sedgwick, ${ }^{\mathrm{a}}$ Günter Haufe, ${ }^{\mathrm{c}}$ Pablo \\ Barrio*,a $^{*}$ and Santos Fustero*,a,b \\ a Departamento de Química Orgánica. Universidad de Valencia, E-46100 Burjassot, \\ Spain. \\ b Laboratorio de Moléculas Orgánicas, Centro de Investigación Príncipe Felipe, E- \\ 46012 Valencia, Spain. \\ ${ }^{\mathrm{c}}$ Organisch-Chemisches Institut, Westfälische Wilhelms-Universität, Corrensstrasse 40, \\ D-48149 Münster, Germany \\ *santos.fustero@uv.es \\ pablo.barrio@uv.es
}

\section{Content:}

I. General methods

II. Experimental procedures and characterization of new compounds............S2-31

III. $\quad{ }^{1} \mathrm{H},{ }^{19} \mathrm{~F}$ and ${ }^{13} \mathrm{C}$ NMR spectra of new compounds.........................S32-131

IV. Chiral HPLC chromatograms of compounds 3a,e, 11a and 20a,e........S132-138 
General methods: All reactions were carried out under a nitrogen atmosphere unless otherwise indicated. Solvents were purified prior to use: THF and $\mathrm{PhMe}$ were distilled from sodium and $\mathrm{CH}_{2} \mathrm{Cl}_{2}$ from calcium hydride. The reactions were monitored with the aid of TLC on $0.25 \mathrm{~mm}$ pre-coated silica-gel plates. Visualization was carried out with UV light and aqueous ceric ammonium molybdate solution or potassium permanganate stain. Flash column chromatography was performed with the indicated solvents on silica gel 60 (particle size: $0.040-0.063 \mathrm{~mm}$ ). ${ }^{1} \mathrm{H},{ }^{13} \mathrm{C}$ and ${ }^{19} \mathrm{~F}$ NMR spectra were recorded on a 300 or $500 \mathrm{MHz}$ spectrometer. Chemical shifts are given in ppm $(\delta)$, referenced to the residual proton resonances of the solvents. Coupling constants $(J)$ are given in Hertz $(\mathrm{Hz})$. The letters $\mathrm{m}, \mathrm{s}, \mathrm{d}, \mathrm{t}$, and $\mathrm{q}$ stand for multiplet, singlet, doublet, triplet, and quartet respectively. The letters bs indicate a broad signal. Enantiomeric excess was determined by means of HPLC (Jasco PU-2089 Plus, detector Jasco MD-2010) using a chiral column OD-H and mixtures of hexane and isopropanol as mobile phase. The words DCM, THF indicate dichloromethane and tetrahydrofuran.

General procedure for the synthesis of compounds 14.<smiles>[R]c1ccc(C=C)c(C=O)c1</smiles>

13a-g
1. (R)- $\mathrm{NH}_{2} \mathrm{SOtBu}, \mathrm{Ti}(\mathrm{OEt})_{4}, \mathrm{DCM}, \mathrm{rt}$

2. $\mathrm{ZnBr}, \mathrm{THF},-40^{\circ} \mathrm{C}$

3. Grubbs II, DCM, rt<smiles>[R]c1ccc2c(c1)[C@H](NS(=O)C(C)(C)C)CC=C2</smiles>

$14 a-g$

A solution of the corresponding aldehyde ( $0.5 \mathrm{mmol}, 1.0$ equiv) and $\mathrm{Ti}(\mathrm{OEt}) 4(2.5$ mmol, 4.0 equiv) in dichloromethane $(5 \mathrm{ml}, 0.1 \mathrm{M})$ was stirred for 5 minutes at room temperature. To the resulting solution $(R)$ - $N$-(tert-butanesulfinyl)amine (0.6 mmol, 1.2 equiv) was added and the mixture was left to stir at room temperature for 12 hours. After this time, saturated aqueous $\mathrm{NaHCO}_{3}$ was added until the white titanium salts precipitated. The suspension was filtered through a short pad of Celite ${ }^{\circledR}$ and washed with small portions of dichloromethane. The filtrate was extracted with ethyl acetate and the combined organic layers were washed with brine, dried over anhydrous $\mathrm{Na}_{2} \mathrm{SO}_{4}$ and concentrated under vacuum. The crude product was used in the next step without any purification. 
Subsequently, a 1M solution of allylzinc bromide in THF was prepared by stirring allyl bromide ( $8.3 \mathrm{mmol}, 1.0$ equiv) and activated $\mathrm{Zn}(30.5 \mathrm{mmol}, 3$ equiv) in anhydrous THF $(8.3 \mathrm{ml}, 1 \mathrm{M})$ at $55{ }^{\circ} \mathrm{C} .1 .1$ equiv $(0.33 \mathrm{mmol}, 0.33 \mathrm{~mL})$ of this freshly prepared solution were added to the residue obtained from the previous step dissolved in THF (3 $\mathrm{ml}, 0.1 \mathrm{M}$ ) at $-40^{\circ} \mathrm{C}$. After stirring 30 minutes (until TLC revealed the disappearance of the starting material) at $-40{ }^{\circ} \mathrm{C}$ the reaction mixture was quenched with saturated $\mathrm{NH}_{4} \mathrm{Cl}$ aq. and extracted three times with AcOEt, dried over anhydrous $\mathrm{Na}_{2} \mathrm{SO}_{4}$ and concentrated under vacuum.

Finally, to a solution of the corresponding allylated compound in $\operatorname{DCM}(2 \mathrm{ml}, 0.1 \mathrm{M})$ $2^{\text {nd }}$ generation Grubbs catalyst $(0.01 \mathrm{mmol}, 5 \mathrm{~mol} \%)$ was added and the mixture was stirred at room temperature for 12 hours. $2^{\text {nd }}$ generation Grubbs catalyst $(0.01 \mathrm{mmol}, 5$ mol\%) was added again and stirred at room temperature for a further 10 hours. Once again, a third portion of $2^{\text {nd }}$ generation Grubbs catalyst $(0.01 \mathrm{mmol}, 5 \mathrm{~mol} \%)$ was added followed by another 6 hours stirring. After that time, the reaction mixture was concentrated under vacuum and purified by means of flash chromatography on silica gel employing mixtures of $n$-hexane: ethyl acetate as eluents.

Compounds 14a-d are described in Org. Lett. 2013, 15, 3770.

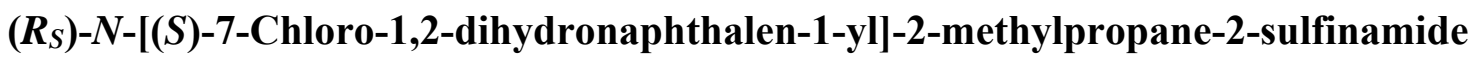
(14e)<smiles>CC(C)(C)[S@@](=O)N[C@H]1CC=Cc2ccc(Cl)cc21</smiles>

Flash chromatography of the crude reaction mixture [ $n$-hexane:EtOAc (1:1)] afforded 14e as a brown oil $(210 \mathrm{mg}, 71 \%, \mathrm{dr}>20: 1)$. $[\alpha]_{\mathrm{D}}{ }^{25}=+63.0\left(\mathrm{c} 1.0 ; \mathrm{CHCl}_{3}\right) .{ }^{1} \mathrm{H}$ NMR $(300$ $\left.\mathrm{MHz}, \mathrm{CDCl}_{3}\right) \delta 1.19(\mathrm{~s}, 9 \mathrm{H}), 2.49-2.84(\mathrm{~m}, 2 \mathrm{H}), 3.42(\mathrm{~d}, J=9.4 \mathrm{~Hz}, 1 \mathrm{H}), 4.44(\mathrm{dd}, J=$ $15.2,8.5 \mathrm{~Hz}, 1 \mathrm{H}), 5.95-6.04(\mathrm{~m}, 1 \mathrm{H}), 6.47$ (d, $J=9.6 \mathrm{~Hz}, 1 \mathrm{H}), 7.00(\mathrm{~d}, J=8.1 \mathrm{~Hz}, 1 \mathrm{H})$, $7.19(\mathrm{dd}, J=8.1,2.2 \mathrm{~Hz}, 1 \mathrm{H}), 7.31(\mathrm{~d}, J=2.1 \mathrm{~Hz}, 1 \mathrm{H}) .{ }^{13} \mathrm{C} \mathrm{NMR}\left(75.5 \mathrm{MHz}, \mathrm{CDCl}_{3}\right)$ $\delta 22.7\left(3 \mathrm{xCH}_{3}\right), 32.5\left(\mathrm{CH}_{2}\right), 54.5(\mathrm{CH}), 56.3(\mathrm{C}), 126.2(\mathrm{CH}), 126.8(\mathrm{CH}), 126.9(\mathrm{CH})$, $127.7(\mathrm{CH}), 128.0(\mathrm{CH}), 131.8$ (C), 132.7 (C), 137.3 (C).HRMS (EI) calcd for $\mathrm{C}_{14} \mathrm{H}_{18} \mathrm{NOSCl}[\mathrm{M}+\mathrm{H}]^{+}: 284.0873$, found: 284.0870 . 
<smiles>Cc1ccc2c(c1)[C@@H](NS(=O)C(C)(C)C)CC=C2</smiles>

Flash chromatography of the crude reaction mixture [ $n$-hexane:EtOAc $(1: 1)]$ afforded $\mathbf{1 4 f}$ as a brown oil $(150 \mathrm{mg}, 52 \%, \mathrm{dr}>20: 1)$. $[\alpha] \mathrm{D}^{25}=+12.6\left(\mathrm{c} 1.0 ; \mathrm{CHCl}_{3}\right) .{ }^{1} \mathrm{H}$ NMR $(300$ $\left.\mathrm{MHz}_{\mathrm{CDCl}}\right) \delta 1.18(\mathrm{~s}, 9 \mathrm{H}), 2.34(\mathrm{~s}, 3 \mathrm{H}), 2.57-2.80(\mathrm{~m}, 2 \mathrm{H}), 3.42(\mathrm{~d}, J=9.2 \mathrm{~Hz}, 1 \mathrm{H})$, $4.44(\mathrm{dt}, J=9.2,6.4 \mathrm{~Hz}, 1 \mathrm{H}), 5.88-5.98(\mathrm{~m}, 1 \mathrm{H}), 6.50(\mathrm{~d}, J=9.6 \mathrm{~Hz}, 1 \mathrm{H}), 6.99$ (d, $J=$ $7.6 \mathrm{~Hz}, 1 \mathrm{H}), 7.04-7.11(\mathrm{~m}, 1 \mathrm{H}), 7.14(\mathrm{~d}, J=0.6 \mathrm{~Hz}, 1 \mathrm{H}) .{ }^{13} \mathrm{C} \mathrm{NMR}\left(75.5 \mathrm{MHz}, \mathrm{CDCl}_{3}\right)$ $\delta 21.6\left(\mathrm{CH}_{3}\right), 22.8\left(3 \mathrm{xCH}_{3}\right), 32.8\left(\mathrm{CH}_{2}\right), 54.7(\mathrm{CH}), 56.3(\mathrm{C}), 124.6(\mathrm{CH}), 126.6(\mathrm{CH})$, $127.8(\mathrm{CH}), 127.9(\mathrm{CH}), 128.8(\mathrm{CH}), 130.8(\mathrm{C}), 135.3(\mathrm{C}), 137.4(\mathrm{C})$. HRMS (EI) calcd for $\mathrm{C}_{15} \mathrm{H}_{21} \mathrm{NOS}[\mathrm{M}+\mathrm{H}]^{+}: 264.1415$, found: 264.1417 .

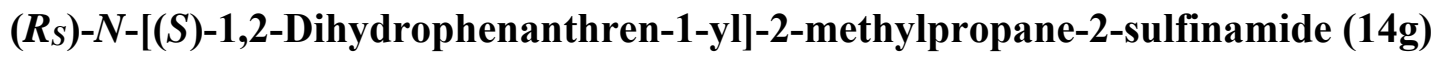<smiles>CC(C)(C)[S@@](=O)N[C@H]1CC=Cc2c1ccc1ccccc21</smiles>

Flash chromatography of the crude reaction mixture [ $n$-hexane:EtOAc (1:1)] afforded 14g as a brown oil $(110 \mathrm{mg}, 72 \%, \mathrm{dr} 16: 1) .[\alpha]_{\mathrm{D}}{ }^{25}=-13.8\left(\mathrm{c} 1.0 ; \mathrm{CHCl}_{3}\right) .{ }^{1} \mathrm{H} \mathrm{NMR}(300 \mathrm{MHz}$, $\left.\mathrm{CDCl}_{3}\right) \delta 1.15(\mathrm{~s}, 9 \mathrm{H}), 3.56(\mathrm{~d}, J=9.7 \mathrm{~Hz}, 1 \mathrm{H}), 4.48-4.65(\mathrm{~m}, 1 \mathrm{H}), 6.19-6.31(\mathrm{~m}, 1 \mathrm{H})$, $7.37(\mathrm{dd}, J=9.9,2.1 \mathrm{~Hz}, 1 \mathrm{H}), 7.42-7.57(\mathrm{~m}, 3 \mathrm{H}), 7.74(\mathrm{~d}, J=8.3 \mathrm{~Hz}, 1 \mathrm{H}), 7.80-7.87$ $(\mathrm{m}, 1 \mathrm{H}), 8.16(\mathrm{~d}, J=8.3 \mathrm{~Hz}, 1 \mathrm{H}) .{ }^{13} \mathrm{C} \mathrm{NMR}\left(75.5 \mathrm{MHz}, \mathrm{CDCl}_{3}\right) \delta 22.6\left(3 \mathrm{xCH}_{3}\right), 32.1$ $\left(\mathrm{CH}_{2}\right), 55.1(\mathrm{CH}), 56.2(\mathrm{C}), 122.9(\mathrm{CH}), 123.1(\mathrm{CH}), 125.8(\mathrm{CH}), 126.0(\mathrm{CH}), 126.2(\mathrm{CH})$, $126.3(\mathrm{CH}), 127.7(\mathrm{CH}), 128.4(\mathrm{C}), 128.5(\mathrm{CH}), 129.4(\mathrm{C}), 132.2(\mathrm{C}), 133.5(\mathrm{C})$. HRMS (EI) calcd for $\mathrm{C}_{18} \mathrm{H}_{21} \mathrm{NOS}[\mathrm{M}+\mathrm{H}]^{+}: 300.1417$, found: 300.1407 . 


\section{Preliminary experiments}

\section{Procedure for the protecting group exchange}<smiles>CC(C)(C)[S@@](=O)N[C@H]1CC=Cc2ccccc21</smiles>

$14 a$
$\mathrm{HCl}, \mathrm{MeOH}, 0^{\circ} \mathrm{C}$ quantitative ran $4 a$

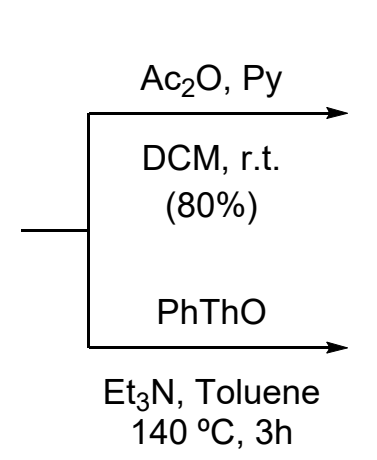

$(44 \%)$<smiles>CC(C)NC1CC=Cc2ccccc21</smiles>

$1 a$<smiles>[NH2+][C@@H]1CC=Cc2ccccc21</smiles>

$7 a$<smiles>O=c1c(=O)c2ccccc12</smiles>

Hydrogen chloride (10.0 equiv, $4 \mathrm{M}$ solution in 1,4-dioxane) was added at $0^{\circ} \mathrm{C}$ to a solution of the corresponding sulfinyl amine ( $2.6 \mathrm{mmol}, 1.0$ equiv) in $\mathrm{MeOH}(2.6 \mathrm{ml}, 1$ M). After 30 minutes the deprotection was complete and the solution was concentrated to dryness. The residue was re-dissolved in $\mathrm{CH}_{2} \mathrm{Cl}_{2}(10 \mathrm{ml})$ and $2 \mathrm{M} \mathrm{NaOH}$ was added and extracted three times with dichloromethane and the combined organic layers were dried over anhydrous $\mathrm{Na}_{2} \mathrm{SO}_{4}$. Solvents were removed under reduced pressure affording 4a, which required no further purification.

Compound 4a is described in Org. Lett. 2013, 15, 3770, as the corresponding hydrochloride.

\section{$N$-Acetyl-protection}

Crude $4 \mathbf{a}$ was dissolved in a mixture of anhydrous DCM/pyridine/Ac $\mathrm{C}_{2} \mathrm{O}(59 \mathrm{ml}, 0.044 \mathrm{M}$ 10/1.5/1 respectively) at room temperature. After 3 hours the reaction mixture was diluted with $\mathrm{CH}_{2} \mathrm{Cl}_{2}$ and the residue was washed consecutively with $5 \mathrm{ml}$ of $\mathrm{HCl}(5 \%), 5 \mathrm{ml}$ of $\mathrm{NaOH}$ (aq.) and $5 \mathrm{ml}$ of water. The combined organic layers were dried over anhydrous $\mathrm{Na}_{2} \mathrm{SO}_{4}$. Solvents were removed under reduced pressure and the residue was purified by means of flash chromatography on silica gel (1:1 n-hexane:ethyl acetate) affording 1a as a colorless oil (390 mg, 80\%).

Compound 1a is described in Org. Lett. 2013, 15, 3770. 


\section{N-Phthalimide-protection}

To a solution of crude $4 \mathbf{a}$ in anhydrous toluene $(0.06 \mathrm{M})$, phthalic anhydride (1 equiv) dissolved in anhydrous toluene $(0.06 \mathrm{M})$ was added. The reaction mixture was heated at $140{ }^{\circ} \mathrm{C}$ and stirred at this temperature overnight. Then, the solvent was removed under reduced pressure and the residue purified by flash chromatography on silica gel (1:1 hexane:ethyl acetate) affording $7 \mathbf{a}$ as a white solid (314mg, 44\%).

\section{(S)-2-(1,2-dihydronaphthalen-1-yl)isoindoline-1,3-dione (7a)}

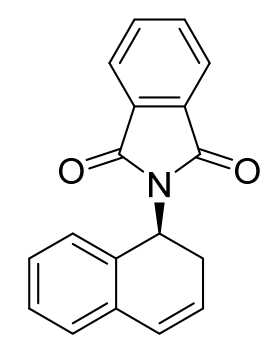

${ }^{1} \mathrm{H}$ NMR $\left(300 \mathrm{MHz}, \mathrm{CDCl}_{3}\right) \delta 2.47$ (dddd, $\left.J=16.8,7.7,5.9,0.9 \mathrm{~Hz}, 1 \mathrm{H}\right), 3.22(\mathrm{ddt}, J=$ 16.7, 13.7, 2.9 Hz, 1H), 5.77 (dd, $J=13.7,7.8 \mathrm{~Hz}, 1 \mathrm{H}), 5.97-6.12(\mathrm{~m}, 1 \mathrm{H}), 6.53$ (dd, $J$ $=9.8,2.8 \mathrm{~Hz}, 1 \mathrm{H}), 6.90(\mathrm{dd}, J=8.1,0.6 \mathrm{~Hz}, 1 \mathrm{H}), 7.07-7.14(\mathrm{~m}, 2 \mathrm{H}), 7.20(\mathrm{ddd}, J=8.1$, 2.1, $1.1 \mathrm{~Hz}, 1 \mathrm{H}), 7.70-7.80(\mathrm{~m}, 2 \mathrm{H}), 7.84-7.93(\mathrm{~m}, 2 \mathrm{H})$.

\section{Procedure for bromofluorination of 1a, $4 a$ and $7 a$}

To a solution of the corresponding 1-amino-1,2-dihydronaphthalene derivative in $\mathrm{CH}_{2} \mathrm{Cl}_{2}$ $(0.5 \mathrm{M}), \mathrm{HF} \cdot \mathrm{Py}$ was added at $0^{\circ} \mathrm{C}$ ( 3 equiv), followed by $N$-bromosuccinimide (1.1 equiv). After stirring at $0^{\circ} \mathrm{C}$ for $15 \mathrm{~min}$, the reaction mixture was allowed to reach room temperature and was left to stir overnight. The resulting solution was poured onto slightly basic ice/water adjusted with $\mathrm{NH}_{4} \mathrm{OH}$, extracted with $\mathrm{CH}_{2} \mathrm{Cl}_{2}$ eight times, washed with $\mathrm{NaHCO}_{3}$, dried over $\mathrm{Na}_{2} \mathrm{SO}_{4}$ and concentrated under reduced pressure.

$N$-((1S,3S,4S)-3-bromo-4-fluoro-1,2,3,4-tetrahydronaphthalen-1-yl)acetamide (2a)<smiles>CC(=O)N[C@H]1C[C@H](Br)[C@H](F)c2ccccc21</smiles> 
Compound 2a was obtained from $74 \mathrm{mg}$ of $1 \mathbf{a}(0.4 \mathrm{mmol})$ as a brown oil (52 $\mathrm{mg}, 45 \%$, $\mathrm{dr}$ $>20: 1) .{ }^{1} \mathrm{H}$ NMR (300 MHz, $\left.\mathrm{CDCl}_{3}\right) \delta 1.94(\mathrm{~s}, 3 \mathrm{H}), 2.47$ - $2.36(\mathrm{~m}, 2 \mathrm{H}), 4.60-4.47$ (m, $1 \mathrm{H}), 5.39-5.27(\mathrm{~m}, 1 \mathrm{H}), 5.54(\mathrm{dd}, J=50.6,5.6 \mathrm{~Hz}, 1 \mathrm{H}), 6.39(\mathrm{~d}, J=8.3 \mathrm{~Hz}, 1 \mathrm{H}), 7.45$ $-7.28(\mathrm{~m}, 4 \mathrm{H}) .{ }^{19} \mathrm{~F}$ NMR $\left(282 \mathrm{MHz}, \mathrm{CDCl}_{3}\right) \delta-150.94(\mathrm{dd}, J=50.6,9.5 \mathrm{~Hz})$.

(1S,3S,4S)-3-Bromo-4-fluoro-1,2,3,4-tetrahydronaphthalen-1-amine (5a)<smiles>NC1C[C@H](Br)C(F)c2ccccc21</smiles>

Compound 5a was obtained from $58 \mathrm{mg}$ of $\mathbf{4 a}(0.4 \mathrm{mmol})$ as an unpurified crude brown

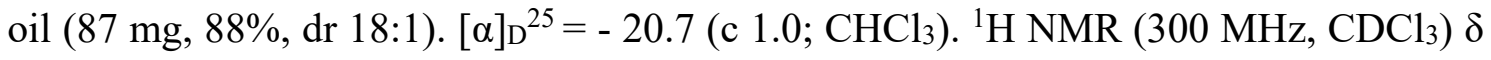
$2.19-2.55$ (m, 4H), 4.22 (dt, $J=7.1,4.8 \mathrm{~Hz}, 1 \mathrm{H}), 4.69$ (tdd, $J=10.7,5.4,3.2 \mathrm{~Hz}, 1 \mathrm{H})$, $5.56(\mathrm{dd}, J=50.6,5.3 \mathrm{~Hz}, 1 \mathrm{H}), 7.29-7.37$ (m, 1H), $7.37-7.47$ (m, 2H), 7.54 (d, $J=7.5$ $\mathrm{Hz}, 1 \mathrm{H}) .{ }^{19} \mathrm{~F}$ NMR $\left(282 \mathrm{MHz}, \mathrm{CDCl}_{3}\right) \delta-149.54(\mathrm{dd}, J=50.6,9.8 \mathrm{~Hz}) .{ }^{13} \mathrm{C}$ NMR $(75.5$ $\left.\mathrm{MHz}, \mathrm{CDCl}_{3}\right) \delta 38.22\left(\mathrm{CH}_{2}\right), 46.48\left(\mathrm{~d},{ }^{2} J_{C F}=24.5 \mathrm{~Hz}, \mathrm{CH}\right), 48.06(\mathrm{CH}), 91.58\left(\mathrm{~d},{ }^{1} J_{C F}=\right.$ $176.5 \mathrm{~Hz}, \mathrm{CH}), 127.64(\mathrm{CH}), 127.88\left(\mathrm{~d},{ }^{4} J_{C F}=1.9 \mathrm{~Hz}, \mathrm{CH}\right), 129.65\left(\mathrm{~d},{ }^{3} J_{C F}=4.7 \mathrm{~Hz}\right.$, $\mathrm{CH}), 130.07\left(\mathrm{~d},{ }^{4} J_{C F}=3.4 \mathrm{~Hz}, \mathrm{CH}\right), 130.99\left(\mathrm{~d},{ }^{2} J_{C F}=17.5 \mathrm{~Hz}, \mathrm{C}\right), 140.21\left(\mathrm{~d},{ }^{3} J_{C F}=3.4\right.$ $\mathrm{Hz}, \mathrm{C})$. HRMS (EI) calcd for $\mathrm{C}_{15} \mathrm{H}_{21} \mathrm{NO}_{2} \mathrm{~S}[\mathrm{M}+\mathrm{H}]^{+}:$280.1364, found: 280.1366.

\section{2-(3-bromo-4-fluoro-1,2,3,4-tetrahydronaphthalen-1-yl)isoindoline-1,3-dione (8a)}<smiles>O=C1c2ccccc2C(=O)N1[C@@H]1C[C@H](Br)C(F)c2ccccc21</smiles>

Compound 8a was obtained from 55mg of 7a (0.2 mmol) as a brown oil (33 mg, 44\%, dr $>20: 1) .{ }^{1} \mathrm{H}$ NMR (300 MHz, $\left.\mathrm{CDCl}_{3}\right) \delta 2.33-2.45$ (m, 1H), 3.26 (dddd, $J=14.2,9.7,2.9$, $1.6 \mathrm{~Hz}, 1 \mathrm{H}), 4.80-4.88(\mathrm{~m}, 1 \mathrm{H}), 5.63(\mathrm{dd}, J=50.1,3.9 \mathrm{~Hz}, 1 \mathrm{H}), 7.03-7.08(\mathrm{~m}, 1 \mathrm{H})$, $5.78-5.88(\mathrm{~m}, 1 \mathrm{H}), 7.29-7.38(\mathrm{~m}, 2 \mathrm{H}), 7.48-7.54(\mathrm{~m}, 1 \mathrm{H}), 7.72-7.78(\mathrm{~m}, 2 \mathrm{H}), 7.83$ $-7.89(\mathrm{~m}, 2 \mathrm{H}) .{ }^{19} \mathrm{~F} \mathrm{NMR}\left(282 \mathrm{MHz}, \mathrm{CDCl}_{3}\right) \delta-142.09--142.39(\mathrm{~m})$. 
Optimization for the base-mediated elimination on acetylated substrates.

\begin{tabular}{|c|c|c|c|c|}
\hline Entry & Base & Solvent & Temp (ㅇ) & Yield (\%) \\
\hline 1 & $\mathrm{KO}^{\mathrm{t}} \mathrm{Bu}$ & THF & $\mathrm{rt}$ & $50 \%$ \\
\hline 2 & $\mathrm{KO}^{\mathrm{t}} \mathrm{Bu}$ & THF & Reflux & $15 \%$ + decomp. \\
\hline 3 & DBU & THF & $\mathrm{rt}$ & Starting material \\
\hline 4 & DBU & THF & Reflux & $43 \%$ \\
\hline 5 & $\mathrm{Et}_{3} \mathrm{~N}$ & THF & rt & Starting material \\
\hline 6 & $\mathrm{Et}_{3} \mathrm{~N}$ & THF & $100 \stackrel{\circ}{ } \mathrm{C}(\mathrm{MW})$ & Starting material \\
\hline 7 & $\mathrm{NaCO}_{3}$ & THF & rt & Starting material \\
\hline 8 & $\mathrm{NaH}$ & THF & $\mathrm{rt}$ & Starting material \\
\hline 9 & $\mathrm{NaH}$ & DMF & $\mathrm{rt}$ & Decomp. \\
\hline
\end{tabular}

\section{General procedure for the preliminary $\mathrm{t}-\mathrm{BuOK}$ mediated elimination.}

To a solution of the corresponding bromofluoro compound in anhydrous THF $(0.1 \mathrm{M}), \mathrm{t}-$ BuOK ( 2.4 equiv) was added at $0{ }^{\circ} \mathrm{C}$. The reaction was stirred and monitored by TLC analysis. Upon completion, the reaction mixture was diluted with AcOEt and quenched with a saturated solution of $\mathrm{NH}_{4} \mathrm{Cl}$. The organic layer was decanted and the aqueous layer extracted with AcOEt 3 times. The combined organic layers were dried over anhydrous $\mathrm{Na}_{2} \mathrm{SO}_{4}$, filtered and concentrated under reduced pressure. The residue was purified by flash chromatography on silica gel (1:1, hexane:ethyl acetate).

(S)-N-(4-Fluoro-1,2-dihydronaphthalen-1-yl)acetamide (3a)<smiles>CC(=O)NC1CC=C(F)c2ccccc21</smiles>

Starting from $46 \mathrm{mg}$ of $\mathbf{2 a}(0.16 \mathrm{mmol}), \mathbf{3 a}$ was obtained as a colorless oil (30 $\mathrm{mg}, 50 \%)$. $[\alpha]_{D^{25}}^{25}-99.5\left(\mathrm{c} 1.0 ; \mathrm{CHCl}_{3}\right) .{ }^{1} \mathrm{H}$ NMR $\left(300 \mathrm{MHz}, \mathrm{CDCl}_{3}\right) \delta 1.95(\mathrm{~s}, 3 \mathrm{H}), 2.52(\mathrm{ddd}, J=$ 
17.2, 9.9, $5.4 \mathrm{~Hz}, 1 \mathrm{H}), 2.69(\mathrm{dddd}, J=17.1,5.9,5.9,3.7 \mathrm{~Hz}, 1 \mathrm{H}), 5.21$ (dt, $J=8.6,5.8$ Hz, 1H), 5.43 (ddd, $J=13.2,5.6,3.7 \mathrm{~Hz}, 1 \mathrm{H}), 5.90$ (d, $J=6.3 \mathrm{~Hz}, 1 \mathrm{H}), 7.29-7.38$ (m, $3 \mathrm{H}), 7.41-7.47(\mathrm{~m}, 1 \mathrm{H}) .{ }^{19} \mathrm{~F}$ NMR $\left(282 \mathrm{MHz}, \mathrm{CDCl}_{3}\right) \delta-121.61--129.23(\mathrm{CF}) .{ }^{13} \mathrm{C}$ $\operatorname{NMR}\left(75.5 \mathrm{MHz}, \mathrm{CDCl}_{3}\right) \delta 23.4\left(\mathrm{CH}_{3}\right), 28.2\left(\mathrm{~d},{ }^{3} J_{\mathrm{CF}}=7.1 \mathrm{~Hz}, \mathrm{CH}_{2}\right), 45.9(\mathrm{CH}), 100.1$ $\left(\mathrm{d},{ }^{2} J_{C F}=16.4 \mathrm{~Hz}, \mathrm{CH}\right), 121.2\left(\mathrm{~d},{ }^{3} J_{C F}=4.4 \mathrm{~Hz}, \mathrm{CH}\right), 127.4\left(\mathrm{~d},{ }^{4} J_{C F}=3.9 \mathrm{~Hz}, \mathrm{CH}\right), 127.9$ (C), $128.3(\mathrm{CH}), 129.2(\mathrm{CH}), 135.6\left(\mathrm{~d},{ }^{2} J_{C F}=6.4 \mathrm{~Hz}, \mathrm{C}\right), 156.1\left(\mathrm{~d},{ }^{1} J_{C F}=251.6 \mathrm{~Hz}, \mathrm{CF}\right)$, 169.1 (C). HRMS (EI) calcd for $\mathrm{C}_{12} \mathrm{H}_{12} \mathrm{NOF}[\mathrm{M}+\mathrm{H}]^{+}: 206.0973$, found: 206.0976

\section{General procedure for the protection with trifluoroacetyl group.}<smiles>CCOC(C)(F)F</smiles>

To a solution of $\mathbf{5 a}$ and triethylamine in anhydrous $\mathrm{CH}_{2} \mathrm{Cl}_{2}(0.1 \mathrm{M})$, trifluoroacetic anhydride was added at $0^{\circ} \mathrm{C}$. After stirring at $0^{\circ} \mathrm{C}$ for $15 \mathrm{~min}$, the mixture was stirred at room temperature and followed by TLC until the starting material was consumed. The reaction mixture was then quenched with $\mathrm{H}_{2} \mathrm{O}$, extracted three times with $\mathrm{CH}_{2} \mathrm{Cl}_{2}$ and the combined organic layers were washed with a saturated aqueous solution of $\mathrm{NaHCO}_{3}$. Flash chromatography afforded the desired compound $\mathbf{1 0 a}$ as a colorless oil.

General procedure for the improved synthesis of compounds $10 a-g$ through consecutive reactions with a single purification.<smiles>[X]c1ccccc1[C@H]1CC=Cc2ccccc21</smiles>

$14 a-g$
1. $\mathrm{HCl}, \mathrm{MeOH}, 0^{\circ} \mathrm{C}$ 2. HF/py, NBS, DCM, $0^{\circ} \mathrm{C}$ a RT, overnight.

3. $\left(\mathrm{CF}_{3} \mathrm{CO}\right)_{2} \mathrm{O}, \mathrm{Et}_{3} \mathrm{~N}, \mathrm{DCM}$<smiles>[X]c1ccc2c(c1)[C@@H](NC(=O)C(F)(F)F)C[C@@H](Br)[C@H]2F</smiles>

Hydrogen chloride (10.0 equiv, $4 \mathrm{M}$ solution in 1,4-dioxane) was added at $0^{\circ} \mathrm{C}$ to a solution of the corresponding sulfinyl amine (1.0 equiv) in $\mathrm{MeOH}(1 \mathrm{M})$. After 30 minutes the deprotection was completed and the solution was concentrated to dryness. 
The residue was re-dissolved in $\mathrm{CH}_{2} \mathrm{Cl}_{2}(0.1 \mathrm{M})$ and $2 \mathrm{M} \mathrm{NaOH}$ was added and extracted three times with dichloromethane. The combined organic layers were dried over anhydrous $\mathrm{Na}_{2} \mathrm{SO}_{4}$ and solvents were removed under reduced pressure.

Subsequently, HF·Py (3 equiv) was added to a solution of the crude 1-amino-1,2dihydronaphthalene derivative in $\mathrm{CH}_{2} \mathrm{Cl}_{2}(0.5 \mathrm{M})$ at $0^{\circ} \mathrm{C}$, followed by the addition of $N$ bromosuccinimide (1.1 equiv). After stirring at $0^{\circ} \mathrm{C}$ for $15 \mathrm{~min}$, the reaction mixture was allowed to reach room temperature and was left to stir overnight. The resulting solution was poured onto slightly basic ice water adjusted with $\mathrm{NH}_{4} \mathrm{OH}$, extracted with $\mathrm{CH}_{2} \mathrm{Cl}_{2}$ eight times, washed with $\mathrm{NaHCO}_{3}$, dried over $\mathrm{Na}_{2} \mathrm{SO}_{4}$ and concentrated.

Finally, to a solution of bromofluoro intermediate 5 and triethylamine (2.5equiv) in $\mathrm{CH}_{2} \mathrm{Cl}_{2}(0.1 \mathrm{M})$ trifluoroacetic anhydride (5 equiv) was added at $0^{\circ} \mathrm{C}$. The reaction mixture was stirred at $0^{\circ} \mathrm{C}$ for $15 \mathrm{~min}$ and then at room temperature until TLC analysis revealed the disappearance of the starting material. Next, the reaction mixture was quenched with $\mathrm{H}_{2} \mathrm{O}$, extracted three times with $\mathrm{CH}_{2} \mathrm{Cl}_{2}$ and the combined organic layers were washed with a saturated aqueous solution of $\mathrm{NaHCO}_{3}$. Flash chromatography afforded the desired compound.

(Note: a splitting or broadening of some peaks was observed due to the presence of rotamers. In the 13C NMR spectra some peaks are missing for the same reason).

\section{$N$-[(1S,3S,4S)-3-Bromo-4-fluoro-1,2,3,4-tetrahydronaphthalen-1-yl]-2,2,2-} trifluoroacetamide (10a)<smiles>O=C(NC1C[C@H](Br)[C@H](F)c2ccccc21)C(F)(F)F</smiles>

Flash chromatography of the crude reaction product [ $n$-hexane:EtOAc (5:1)] afforded 10a as a colorless oil $(115 \mathrm{mg}, 77 \%, \mathrm{dr} 18: 1) .[\alpha]_{\mathrm{D}}{ }^{25}=-67.5\left(\mathrm{c} 1.0 ; \mathrm{CHCl}_{3}\right) .{ }^{1} \mathrm{H}$ NMR (300 $\left.\mathrm{MHz}, \mathrm{CDCl}_{3}\right) \delta 2.46-2.64(\mathrm{~m}, 2 \mathrm{H}), 4.57(\mathrm{ddd}, J=9.8,7.0,4.4 \mathrm{~Hz}, 1 \mathrm{H}), 5.42-5.69(\mathrm{~m}$, 2H), $6.69(\mathrm{~d}, J=7.4 \mathrm{~Hz}, 1 \mathrm{H}), 7.27-7.52(\mathrm{~m}, 4 \mathrm{H}) .{ }^{19} \mathrm{~F} \mathrm{NMR}\left(282 \mathrm{MHz}, \mathrm{CDCl}_{3}\right) \delta-75.69$ $\left(\mathrm{s}, \mathrm{CF}_{3}\right),-145.31(\mathrm{dd}, J=50.2,5.0 \mathrm{~Hz}, \mathrm{CHF}) .{ }^{13} \mathrm{C} \mathrm{NMR}\left(75.5 \mathrm{MHz}, \mathrm{CDCl}_{3}\right) \delta 33.0\left(\mathrm{CH}_{2}\right)$, $44.3\left(\mathrm{~d},{ }^{2} J_{C F}=25.8 \mathrm{~Hz}, \mathrm{CH}\right), 46.7(\mathrm{CH}), 89.9\left(\mathrm{~d},{ }^{1} J_{C F}=176.4 \mathrm{~Hz}, \mathrm{CH}\right), 115.8\left(\mathrm{q},{ }^{1} J_{C F}=\right.$ $287.8 \mathrm{~Hz}, \mathrm{C}), 127.4(\mathrm{CH}), 129.1\left(\mathrm{~d},{ }^{4} J_{C F}=2.3 \mathrm{~Hz}, \mathrm{CH}\right), 130.6(\mathrm{CH}), 130.7\left(\mathrm{~d},{ }^{3} J_{C F}=4.1\right.$ 
$\mathrm{Hz}, \mathrm{CH}), 131.2\left(\mathrm{~d},{ }^{2} J_{C F}=17.5 \mathrm{~Hz}, \mathrm{C}\right), 134.1\left(\mathrm{~d},{ }^{3} J_{C F}=3.5 \mathrm{~Hz}, \mathrm{C}\right), 157.1\left(\mathrm{q},{ }^{2} J_{C F}=37.5\right.$ $\mathrm{Hz}, \mathrm{C})$. HRMS (EI) calcd for $\mathrm{C}_{12} \mathrm{H}_{10} \mathrm{BrF}_{4} \mathrm{NO}[\mathrm{M}+\mathrm{H}]^{+}: 339.9882$, found: 339.9880 .

\section{$N$-[(1S,3S,4S)-3-Bromo-4,8-difluoro-1,2,3,4-tetrahydronaphthalen-1-yl]-2,2,2-}

\section{trifluoroacetamide $(10 \mathrm{c})$}<smiles>O=C(NC1C[C@H](Br)[C@H](F)c2cccc(F)c21)C(F)(F)F</smiles>

Flash chromatography of the crude reaction product [ $n$-hexane:EtOAc (5:1)] afforded 10c as a brown oil $\left(280 \mathrm{mg}, 52 \%\right.$, dr 9:1). $[\alpha]_{\mathrm{D}}{ }^{25}=-68.4\left(\mathrm{c} 1.0 ; \mathrm{CHCl}_{3}\right) .{ }^{1} \mathrm{H} \mathrm{NMR}(300 \mathrm{MHz}$, $\left.\mathrm{CDCl}_{3}\right) \delta 2.45-2.70(\mathrm{~m}, 2 \mathrm{H}), 4.41-4.54(\mathrm{~m}, 1 \mathrm{H}), 5.42(\mathrm{dd}, J=11.9,5.8 \mathrm{~Hz}, 1 \mathrm{H}), 5.56$ $(\mathrm{dd}, J=50.2,7.0 \mathrm{~Hz}, 1 \mathrm{H}), 6.97(\mathrm{~d}, J=7.4 \mathrm{~Hz}, 1 \mathrm{H}), 7.12(\mathrm{dd}, J=9.7,8.3 \mathrm{~Hz}, 1 \mathrm{H}), 7.32$ $(\mathrm{d}, J=7.6 \mathrm{~Hz}, 1 \mathrm{H}), 7.38-7.47(\mathrm{~m}, 1 \mathrm{H}) .{ }^{19} \mathrm{~F} \mathrm{NMR}\left(282 \mathrm{MHz}, \mathrm{CDCl}_{3}\right) \delta-75.81\left(\mathrm{~s}, \mathrm{CF}_{3}\right)$, $-114.43(\mathrm{dd}, J=9.6,5.3 \mathrm{~Hz}, \mathrm{CF}),-161.21(\mathrm{dd}, J=50.1,9.6 \mathrm{~Hz}, \mathrm{CHF}) .{ }^{13} \mathrm{C}$ NMR $(75.5$ $\left.\mathrm{MHz}, \mathrm{CDCl}_{3}\right) \delta 34.73\left(\mathrm{~d},{ }^{3} J_{C F}=3.3 \mathrm{~Hz}, \mathrm{CH}_{2}\right), 43.24(\mathrm{CH}), 43.82\left(\mathrm{~d},{ }^{2} J_{C F}=22.2 \mathrm{~Hz}, \mathrm{CH}\right)$, $90.88\left(\mathrm{~d},{ }^{1} J_{C F}=181.0 \mathrm{~Hz}, \mathrm{CHF}\right), 115.60\left(\mathrm{q},{ }^{1} J_{C F}=287.9 \mathrm{~Hz}, \mathrm{C}\right), 116.91\left(\mathrm{dd},{ }^{2} J_{C F}=21.1\right.$, $\left.{ }^{5} J_{C F}=1.9 \mathrm{~Hz}, \mathrm{CH}\right), 120.75\left(\mathrm{dd},{ }^{2} J_{C F}=15.0,{ }^{3} J_{C F}=3.2 \mathrm{~Hz}, \mathrm{C}\right), 124.49(\mathrm{CH}), 130.99(\mathrm{~d}$, $\left.{ }^{3} J_{C F}=8.6 \mathrm{~Hz}, \mathrm{CH}\right), 135.18\left(\mathrm{dd},{ }^{2} J_{C F}=18.8,{ }^{3} J_{C F}=2.4 \mathrm{~Hz}, \mathrm{C}\right), 156.58\left(\mathrm{q},{ }^{2} J_{C F}=37.7 \mathrm{~Hz}\right.$, C), $160.57\left(\mathrm{~d},{ }^{1} J_{C F}=251.3 \mathrm{~Hz}, \mathrm{C}\right)$. HRMS (EI) calcd for $\mathrm{C}_{12} \mathrm{H}_{9} \mathrm{BrF}_{5} \mathrm{NO} \quad[\mathrm{M}+\mathrm{H}]^{+}$: 375.0126, found: 375.0126 .

$N$-[(1S,3S,4S)-3-Bromo-4-fluoro-7-(trifluoromethyl)-1,2,3,4-tetrahydronaphthalen1-yl]-2,2,2-trifluoroacetamide (10d)<smiles>O=C(NC1C[C@H](Br)[C@H](F)c2ccc(C(F)(F)F)cc21)C(F)(F)F</smiles>

Flash chromatography of the crude reaction product [n-hexane:EtOAc (5:1)] afforded 10d as a brown oil $(300 \mathrm{mg}, 60 \%, \mathrm{dr} 13: 1) .[\alpha]_{\mathrm{D}}{ }^{25}=-56.1\left(\mathrm{c} 1.0 ; \mathrm{CHCl}_{3}\right) .{ }^{1} \mathrm{H} \mathrm{NMR}(300 \mathrm{MHz}$, $\left.\mathrm{CDCl}_{3}\right) \delta 2.53-2.60(\mathrm{~m}, 2 \mathrm{H}), 4.59(\mathrm{td}, J=9.5,4.6 \mathrm{~Hz}, 1 \mathrm{H}), 5.44-5.71(\mathrm{~m}, 2 \mathrm{H}), 6.90$ $(\mathrm{d}, J=8.5 \mathrm{~Hz}, 1 \mathrm{H}), 7.53(\mathrm{~s}, 1 \mathrm{H}), 7.62$ (d, $J=8.3 \mathrm{~Hz}, 1 \mathrm{H}), 7.68(\mathrm{~d}, J=8.2 \mathrm{~Hz}, 1 \mathrm{H}) .{ }^{19} \mathrm{~F}$ 
$\operatorname{NMR}\left(282 \mathrm{MHz}, \mathrm{CDCl}_{3}\right) \delta-63.12\left(\mathrm{~s}, \mathrm{CF}_{3}\right),-75.74\left(\mathrm{~s}, \mathrm{CF}_{3}\right),-146.21(\mathrm{~d}, J=49.3 \mathrm{~Hz}, \mathrm{CHF})$. ${ }^{13} \mathrm{C} \mathrm{NMR}\left(75.5 \mathrm{MHz}, \mathrm{CDCl}_{3}\right) \delta 32.66\left(\mathrm{CH}_{2}\right), 43.55\left(\mathrm{~d},{ }^{2} J_{C F}=25.5 \mathrm{~Hz}, \mathrm{CH}\right), 46.39(\mathrm{CH})$, $89.05\left(\mathrm{~d},{ }^{1} J_{C H}=177.9 \mathrm{~Hz}, \mathrm{CH}\right), 115.62\left(\mathrm{q},{ }^{1} J_{C F}=287.8 \mathrm{~Hz}, \mathrm{C}\right), 123.30\left(\mathrm{q},{ }^{1} J_{C F}=272.9\right.$ $\mathrm{Hz}, \mathrm{C}), 124.37(\mathrm{CH}), 125.80(\mathrm{CH}), 131.42\left(\mathrm{~d},{ }^{3} J_{C F}=4.0 \mathrm{~Hz}, \mathrm{CH}\right), 132.86\left(\mathrm{q},{ }^{2} J_{C F}=33.1\right.$ $\mathrm{Hz}, \mathrm{C}), 134.71\left(\mathrm{~d},{ }^{2} J_{C F}=17.3 \mathrm{~Hz}, \mathrm{C}\right), 135.30\left(\mathrm{~d},{ }^{3} J_{C F}=3.2 \mathrm{~Hz}, \mathrm{C}\right), 157.38\left(\mathrm{q},{ }^{2} J_{C F}=37.9\right.$ $\mathrm{Hz}, \mathrm{C})$. HRMS (EI) calcd for $\mathrm{C}_{13} \mathrm{H}_{9} \mathrm{NOF}_{7} \mathrm{Br}\left[\mathrm{M}+\mathrm{NH}_{4}\right]^{+}: 425.0095$, found: 425.0094 .

$N$-[(1S,3S,4S)-3-Bromo-7-chloro-4-fluoro-1,2,3,4-tetrahydronaphthalen-1-yl]-2,2,2trifluoroacetamide (10e)<smiles>O=C(N[C@H]1C[C@H](Br)[C@H](F)c2ccc(Cl)cc21)C(F)(F)F</smiles>

Flash chromatography of the crude reaction product [ $n$-hexane:EtOAc (5:1)] afforded 10e as a colorless oil $(42 \mathrm{mg}, 65 \% \text {, dr 15:1). [ } \alpha]_{\mathrm{D}}{ }^{25}=-161.0\left(\mathrm{c} 1.0 ; \mathrm{CHCl}_{3}\right) .{ }^{1} \mathrm{H}$ NMR (300 $\left.\mathrm{MHz}, \mathrm{CDCl}_{3}\right) \delta 2.43-2.61(\mathrm{~m}, 2 \mathrm{H}), 4.51-4.62(\mathrm{~m}, 1 \mathrm{H}), 5.38-5.64(\mathrm{~m}, 2 \mathrm{H}), 6.83(\mathrm{~d}, J$ $=8.4 \mathrm{~Hz}, 1 \mathrm{H}), 7.21-7.32(\mathrm{~m}, 1 \mathrm{H}), 7.35-7.45(\mathrm{~m}, 2 \mathrm{H}) .{ }^{19} \mathrm{~F} \mathrm{NMR}\left(282 \mathrm{MHz}, \mathrm{CDCl}_{3}\right) \delta$ $-75.61\left(\mathrm{~s}, \mathrm{CF}_{3}\right),-143.38$ (ddd, $\left.J=50.2,9.0,5.0 \mathrm{~Hz}, \mathrm{CHF}\right) .{ }^{13} \mathrm{C} \mathrm{NMR}\left(75.5 \mathrm{MHz}, \mathrm{CDCl}_{3}\right)$ $\delta 32.5\left(\mathrm{CH}_{2}\right), 43.9\left(\mathrm{~d},{ }^{2} J_{C F}=26.2 \mathrm{~Hz}, \mathrm{CH}\right), 46.2(\mathrm{CH}), 89.1\left(\mathrm{~d},{ }^{1} J_{C F}=176.8 \mathrm{~Hz}, \mathrm{CH}\right)$, $115.7\left(\mathrm{q},{ }^{1} J_{C F}=288.0 \mathrm{~Hz}, \mathrm{C}\right), 127.3\left(\mathrm{~d},{ }^{4} J_{C F}=1.4 \mathrm{~Hz}\right), 129.4\left(\mathrm{~d},{ }^{4} J_{C F}=2.5 \mathrm{~Hz}, \mathrm{CH}\right)$, $129.4\left(\mathrm{~d},{ }^{2} J_{C F}=17.5 \mathrm{~Hz}, \mathrm{C}\right), 132.3\left(\mathrm{~d},{ }^{3} J_{C F}=3.8 \mathrm{~Hz}, \mathrm{CH}\right), 136.2\left(\mathrm{~d},{ }^{5} J_{C F}=3.4 \mathrm{~Hz}, \mathrm{C}\right)$, $136.8\left(\mathrm{~d},{ }^{3} J_{C F}=4.4 \mathrm{~Hz}, \mathrm{C}\right), 157.3\left(\mathrm{q},{ }^{2} J_{C F}=37.7 \mathrm{~Hz}, \mathrm{C}\right)$. HRMS (EI) calcd for $\mathrm{C}_{12} \mathrm{H}_{9} \mathrm{NOF}_{4} \mathrm{ClBr}[\mathrm{M}+\mathrm{H}]^{+}: 390.9821$, found: 390.9821 .

\section{$N$-[(1S,3S,4S)-3-Bromo-4-fluoro-7-methyl-1,2,3,4-tetrahydronaphthalen-1-yl]-}

\section{2,2,2-trifluoroacetamide (10f)}<smiles>Cc1ccc2c(c1)[C@H](NC(=O)C(F)(F)F)C[C@@H](Br)[C@H]2F</smiles>

Flash chromatography of the crude reaction product [ $n$-hexane:EtOAc (5:1)] afforded 10f as a colorless oil $\left(80 \mathrm{mg}, 69 \%\right.$, dr 2:1). $[\alpha] \mathrm{D}^{25}=-67.6\left(\mathrm{c} 1.0 ; \mathrm{CHCl}_{3}\right) .{ }^{1} \mathrm{H} \mathrm{NMR}(300 \mathrm{MHz}$, 
$\left.\mathrm{CDCl}_{3}\right) \delta 2.38(\mathrm{~d}, J=2.6 \mathrm{~Hz}, 3 \mathrm{H}) .2 .45-2.64(\mathrm{~m}, 2 \mathrm{H}), 4.50-4.61(\mathrm{~m}, 1 \mathrm{H}), 5.38-5.66$ (m, 2H), 6.59 (d, J=7.6 Hz, 1H), 7.09 (s, 1H), 7.23 (d, J=7.9 Hz, 1H), 7.37 (dd, J = 7.9, $1.4 \mathrm{~Hz}, 1 \mathrm{H}) .{ }^{19} \mathrm{~F}$ NMR $\left(282 \mathrm{MHz}, \mathrm{CDCl}_{3}\right) \delta-75.67\left(\mathrm{~s}, \mathrm{CF}_{3}\right),-144.52(\mathrm{~d}, J=50.1 \mathrm{~Hz}$, CHF). ${ }^{13} \mathrm{C}$ NMR $\left(75.5 \mathrm{MHz}, \mathrm{CDCl}_{3}\right) \delta 30.8\left(\mathrm{CH}_{3}\right), 33.1\left(\mathrm{CH}_{2}\right), 44.5\left(\mathrm{~d},{ }^{2} J_{C F}=26.0 \mathrm{~Hz}\right.$, $\mathrm{CH}), 46.6(\mathrm{CH}), 89.9\left(\mathrm{~d},{ }^{1} J_{C F}=175.8 \mathrm{~Hz}, \mathrm{CH}\right), 115.8\left(\mathrm{q},{ }^{1} J_{C F}=287.8 \mathrm{~Hz}, \mathrm{C}\right), 127.7(\mathrm{~d}$, $\left.{ }^{4} J_{C F}=1.0 \mathrm{~Hz}, \mathrm{CH}\right), 128.3\left(\mathrm{~d},{ }^{2} J_{C F}=17.8 \mathrm{~Hz}, \mathrm{C}\right), 129.9\left(\mathrm{~d},{ }^{4} J_{C F}=2.6 \mathrm{~Hz}, \mathrm{CH}\right), 130.6(\mathrm{~d}$, $\left.{ }^{3} J_{C F}=4.0 \mathrm{~Hz}, \mathrm{CH}\right), 134.0\left(\mathrm{~d},{ }^{5} J_{C F}=3.5 \mathrm{~Hz}, \mathrm{C}\right), 140.9\left(\mathrm{~d},{ }^{4} J_{C F}=4.0 \mathrm{~Hz}, \mathrm{C}\right), 157.1$ (q, $J=$ $37.5 \mathrm{~Hz}$ ). HRMS (EI) calcd for $\mathrm{C}_{13} \mathrm{H}_{12} \mathrm{NOF}_{4} \mathrm{Br}[\mathrm{M}+\mathrm{H}]^{+}: 274.0847$, found: 274.0850.

\section{General procedure for the O-heterocyclization.}

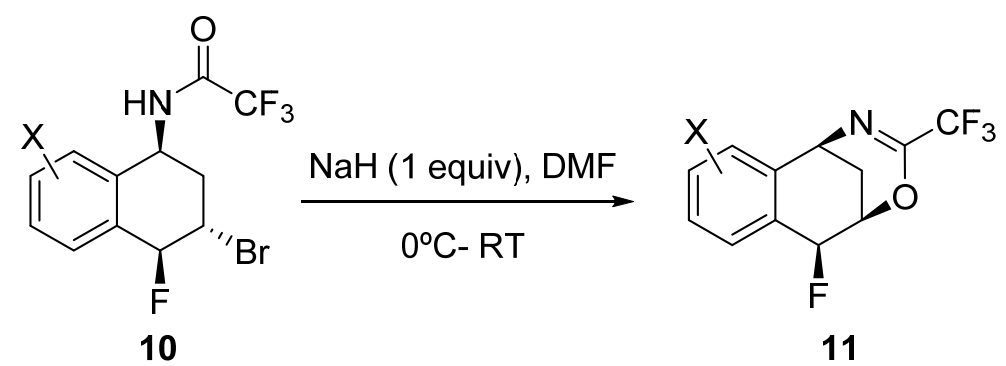

A solution of compound 10 (1.0 equiv) in dry DMF (0.06M) was treated with $\mathrm{NaH}(1.0$ equiv) at $0^{\circ} \mathrm{C}$. The reaction mixture was stirred at $0^{\circ} \mathrm{C}$ for $15 \mathrm{~min}$ and then at room temperature until TLC revealed the disappearance of the starting material. Next, the reaction mixture was quenched with $\mathrm{H}_{2} \mathrm{O}$ and extracted three times with $\mathrm{Et}_{2} \mathrm{O}$. The combined organic layers were washed with $\mathrm{H}_{2} \mathrm{O}$ three times and dried over $\mathrm{Na}_{2} \mathrm{SO}_{4}$. Flash chromatography on silica gel (hexane:ethyl acetate, 3:1) afforded the desired compound.

\section{(1S,5R,6S)-6-Fluoro-3-(trifluoromethyl)-5,6-dihydro-1H-1,5-}

\section{methanobenzo[e][1,3]oxazocine (11a)}<smiles>FC1c2ccccc2C2CC1OC(C(F)(F)F)=N2</smiles>

Following the general procedure, 11a was obtained as a brown oil (25 mg, 88\%, dr $>20: 1)$. $[\alpha]_{\mathrm{D}^{25}}=+84.4\left(\mathrm{c} 1.0 ; \mathrm{CHCl}_{3}\right) .{ }^{1} \mathrm{H}$ NMR $\left(300 \mathrm{MHz}, \mathrm{CDCl}_{3}\right) \delta 2.19-2.27(\mathrm{~m}, 2 \mathrm{H}), 4.79$ (dd, $J=4.6,2.3 \mathrm{~Hz}, 1 \mathrm{H}), 5.17-5.26(\mathrm{~m}, 1 \mathrm{H}), 5.78(\mathrm{dd}, J=47.6,3.8 \mathrm{~Hz}, 1 \mathrm{H}), 7.33-7.45$ (m, 3H), $7.56-7.64(\mathrm{~m}, 1 \mathrm{H}) .{ }^{19} \mathrm{~F}$ NMR $\left(282 \mathrm{MHz}, \mathrm{CDCl}_{3}\right) \delta-73.81\left(\mathrm{~s}, \mathrm{CF}_{3}\right),-187.17(\mathrm{~d}$, $J=47.7 \mathrm{~Hz}, \mathrm{CHF}) .{ }^{13} \mathrm{C} \mathrm{NMR}\left(75.5 \mathrm{MHz}, \mathrm{CDCl}_{3}\right) \delta 24.9\left(\mathrm{~d},{ }^{3} J_{C F}=4.0 \mathrm{~Hz}, \mathrm{CH}_{2}\right), 49.3$ 
$(\mathrm{CH}), 71.9\left(\mathrm{~d},{ }^{2} J_{C F}=15.3 \mathrm{~Hz}, \mathrm{CH}\right), 90.8\left(\mathrm{~d},{ }^{1} J_{C F}=185.2 \mathrm{~Hz}, \mathrm{CH}\right), 116.6\left(\mathrm{q},{ }^{1} J_{C F}=276.3\right.$ $\left.\mathrm{Hz}, \mathrm{CF}_{3}\right), 128.3\left(\mathrm{~d},{ }^{3} J_{C F}=5.1 \mathrm{~Hz}, \mathrm{CH}\right), 128.8(2 \mathrm{xCH}), 129.4(\mathrm{CH}), 130.9\left(\mathrm{~d},{ }^{2} J_{C F}=19.7\right.$ $\mathrm{Hz}, \mathrm{C}), 136.1\left(\mathrm{~d},{ }^{3} J_{C F}=3.1 \mathrm{~Hz}, \mathrm{C}\right), 148.6$ (q, $\left.{ }^{2} J_{C F}=38.8 \mathrm{~Hz}, \mathrm{C}\right)$. HRMS (EI) calcd for $\mathrm{C}_{12} \mathrm{H}_{9} \mathrm{~F}_{4} \mathrm{NO} \quad[\mathrm{M}+\mathrm{H}]^{+}:$260.0694, found: 260.0693. The enantiomeric excess was determined with the aid of HPLC analysis: Chiralcel OD-H (25 cm x $0.46 \mathrm{~cm}$ column), hexane:isopropanol 99:1 as eluent and flow $=1 \mathrm{~mL} / \mathrm{min}: t_{\mathrm{R} \text { major }}=7.00 \mathrm{~min}, t_{\mathrm{R} \text { minor }}=8.65$ $\min$.

(1S,5R,6S)-6,10-Difluoro-3-(trifluoromethyl)-5,6-dihydro-1H-1,5methanobenzo[e][1,3] oxazocine (11c)<smiles>Fc1cccc2c1C1CC(OC(C(F)(F)F)=N1)C2F</smiles>

Following the general procedure, 11c was obtained as a white solid (52 mg, 77\%, dr $>20: 1) \cdot[\alpha]_{\mathrm{D}}{ }^{25}=+119.8\left(\mathrm{c} 1.0 ; \mathrm{CHCl}_{3}\right) \cdot \mathrm{Mp} 140-142^{\circ} \mathrm{C} .{ }^{1} \mathrm{H}$ NMR $\left(300 \mathrm{MHz}, \mathrm{CDCl}_{3}\right) \delta$ $2.12-2.29(\mathrm{~m}, 2 \mathrm{H}), 5.14-5.18(\mathrm{~m}, 1 \mathrm{H}), 5.18-5.25(\mathrm{~m}, 1 \mathrm{H}), 5.75(\mathrm{dd}, J=47.2,3.7 \mathrm{~Hz}$, 1H), $7.05-7.14(\mathrm{~m}, 1 \mathrm{H}), 7.30-7.43(\mathrm{~m}, 2 \mathrm{H}) .{ }^{19} \mathrm{~F}$ NMR $\left(282 \mathrm{MHz}, \mathrm{CDCl}_{3}\right) \delta-73.72$ $\left(\mathrm{CF}_{3}\right),-116.54--120.18(\mathrm{~m}, \mathrm{CF}),-187.00$ (dd, $\left.J=47.3,3.6 \mathrm{~Hz}, \mathrm{CHF}\right) .{ }^{13} \mathrm{C}$ NMR $(75.5$ $\left.\mathrm{MHz} \mathrm{CDCl}_{3}\right) \delta 24.42\left(\mathrm{~d},{ }^{3} J_{C F}=4.3 \mathrm{~Hz}, \mathrm{CH}_{2}\right), 42.31\left(\mathrm{~d},{ }^{4} J_{\mathrm{CF}}=2.5 \mathrm{~Hz}, \mathrm{CH}\right), 71.61(\mathrm{~d}$, $\left.{ }^{2} J_{C F}=15.4 \mathrm{~Hz}, \mathrm{CH}\right), 90.34\left(\mathrm{dd},{ }^{1} J_{C F}=186.1,{ }^{4} J_{C F}=2.6 \mathrm{~Hz}, \mathrm{CH}\right), 116.05\left(\mathrm{~d},{ }^{2} J=20.4\right.$ $\mathrm{Hz}, \mathrm{CH}), 116.58\left(\mathrm{q},{ }^{1} J_{\mathrm{CF}}=276.7 \mathrm{~Hz}, \mathrm{C}\right), 123.60-123.81(\mathrm{~m}, \mathrm{CH}), 124.09\left(\mathrm{dd},{ }^{2} J_{C F}=\right.$ $\left.18.3,{ }^{3} J_{C F}=3.2 \mathrm{~Hz}, \mathrm{C}\right), 129.88\left(\mathrm{~d},{ }^{3} J_{C F}=8.3 \mathrm{~Hz}\right), 133.13\left(\mathrm{dd},{ }^{2} J_{C F}=20.3,{ }^{3} J_{C F}=3.4 \mathrm{~Hz}\right)$, $149.63\left(\mathrm{q},{ }^{2} J_{C F}=39.2 \mathrm{~Hz}, \mathrm{C}\right), 159.35\left(\mathrm{~d},{ }^{1} J_{C F}=249.4 \mathrm{~Hz}, \mathrm{C}\right)$. HRMS (EI) calcd for $\mathrm{C}_{12} \mathrm{H}_{8} \mathrm{~F}_{5} \mathrm{NO}[\mathrm{M}+\mathrm{H}]^{+}: 278.0606$, found: 278.0599 .

\section{(1S,5R,6S)-6-Fluoro-3,9-bis(trifluoromethyl)-5,6-dihydro-1H-1,5-} methanobenzo[e][1,3] oxazocine (11d)<smiles>FC1c2ccc(C(F)(F)F)cc2C2CC1OC(C(F)(F)F)=N2</smiles>

Following the general procedure, 11d was obtained as a colorless oil $(35 \mathrm{mg}, 87 \%$, dr $>20: 1) .[\alpha] \mathrm{D}^{25}=-8.1\left(\mathrm{c} 1.0 ; \mathrm{CHCl}_{3}\right) .{ }^{1} \mathrm{H} \mathrm{NMR}\left(300 \mathrm{MHz}, \mathrm{CDCl}_{3}\right) \delta 2.25-2.29(\mathrm{~m}, 2 \mathrm{H})$, $4.86(\mathrm{dd}, J=4.6,2.3 \mathrm{~Hz}, 1 \mathrm{H}), 5.20-5.29(\mathrm{~m}, 1 \mathrm{H}), 5.78(\mathrm{dd}, J=47.0,3.7 \mathrm{~Hz}, 1 \mathrm{H}), 7.78$ 
- $7.61(\mathrm{~m}, 3 \mathrm{H}) .{ }^{19} \mathrm{~F}$ NMR $\left(282 \mathrm{MHz}, \mathrm{CDCl}_{3}\right) \delta-62.92\left(\mathrm{~s}, \mathrm{CF}_{3}\right),-73.82\left(\mathrm{~s}, \mathrm{CF}_{3}\right),-188.43$ $(\mathrm{d}, J=46.9 \mathrm{~Hz}, \mathrm{CHF}) .{ }^{13} \mathrm{C} \mathrm{NMR}\left(75.5 \mathrm{MHz}, \mathrm{CDCl}_{3}\right) \delta 24.60\left(\mathrm{~d},{ }^{3} J_{C F}=4.0 \mathrm{~Hz}, \mathrm{CH}_{2}\right)$, $48.99(\mathrm{CH}), 71.60\left(\mathrm{~d},{ }^{2} J_{C F}=15.4 \mathrm{~Hz}, \mathrm{CH}\right), 90.33\left(\mathrm{~d},{ }^{1} J_{C F}=186.9 \mathrm{~Hz}, \mathrm{CH}\right), 116.51$ (q, $\left.{ }^{1} J_{C F}=276.6 \mathrm{~Hz}, \mathrm{C}\right), 123.46\left(\mathrm{q},{ }^{1} J_{C F}=272.5 \mathrm{~Hz}, \mathrm{C}\right), 125.60\left(\mathrm{~d},{ }^{4} J_{C F}=2.9 \mathrm{~Hz}, \mathrm{CH}\right), 125.79$ $\left(\mathrm{d},{ }^{4} J_{C F}=3.6 \mathrm{~Hz}, \mathrm{CH}\right), 129.07\left(\mathrm{~d},{ }^{3} J_{C F}=5.2 \mathrm{~Hz}, \mathrm{CH}\right), 131.75\left(\mathrm{q},{ }^{2} J_{C F}=33.3 \mathrm{~Hz}, \mathrm{C}\right)$, $134.80\left(\mathrm{~d},{ }^{2} J_{C F}=19.5 \mathrm{~Hz}, \mathrm{C}\right), 136.83\left(\mathrm{~d},{ }^{3} J_{C F}=2.8 \mathrm{~Hz}, \mathrm{C}\right), 149.04\left(\mathrm{q},{ }^{2} J_{C F}=39.4 \mathrm{~Hz}, \mathrm{C}\right)$. HRMS (EI) calcd for $\mathrm{C}_{11} \mathrm{H}_{11} \mathrm{~F}_{4} \mathrm{NO}$ [M+H-CCF$]^{+}: 250.0853$, found: 250.0853 .

\section{General procedure of synthesis of fluroalkenes 12a,c-f.}<smiles>[X]c1cccc2c1C(F)=CC[C@H]2NC(=O)C(F)(F)F</smiles>

A solution of compound 11 (1.0 equiv) in dry DMF (0.06M) was treated with $\mathrm{NaH}$ (4 equiv) at $0^{\circ} \mathrm{C}$. The reaction mixture was stirred at $0^{\circ} \mathrm{C}$ for $15 \mathrm{~min}$ and then at room temperature until TLC revealed the disappearance of the starting material. Next, the reaction mixture was quenched with $\mathrm{H}_{2} \mathrm{O}$ and extracted three times with $\mathrm{Et}_{2} \mathrm{O}$. The combined organic layers were washed with $\mathrm{H}_{2} \mathrm{O}$ three times and dried over $\mathrm{Na}_{2} \mathrm{SO}_{4}$. Flash chromatography afforded the desired compound.

(Note: a splitting or broadening of some peaks was observed due to rotamers).

(S)-2,2,2-Trifluoro- $N$-(4-fluoro-1,2-dihydronaphthalen-1-yl)acetamide (12a)<smiles>O=C(NC1CC=C(F)c2ccccc21)C(F)(F)F</smiles>

Following the general procedure, 12a was obtained as a white solid $(78 \mathrm{mg},>99 \%)$. $[\alpha]_{\mathrm{D}}{ }^{25}$ $=-151.5\left(\mathrm{c} 1.0 ; \mathrm{CHCl}_{3}\right) . \mathrm{Mp} 100-102{ }^{\circ} \mathrm{C} .{ }^{1} \mathrm{H} \mathrm{NMR}\left(300 \mathrm{MHz}, \mathrm{CDCl}_{3}\right) \delta 2.60(\mathrm{dddd}, J=$ 17.4, 5.6, 4.5, $4.5 \mathrm{~Hz}, 1 \mathrm{H}), 2.78$ (dddd, $J=17.4,5.9,5.9,3.5 \mathrm{~Hz}, 1 \mathrm{H}), 5.22$ (dt, $J=8.6$, $5.5 \mathrm{~Hz}, 1 \mathrm{H}), 5.46$ (ddd, $J=12.9,5.7,3.5 \mathrm{~Hz}, 1 \mathrm{H}), 6.69$ (d, $J=1.6 \mathrm{~Hz}, 1 \mathrm{H}), 7.32-7.37$ 
$(\mathrm{m}, 2 \mathrm{H}), 7.38-7.45(\mathrm{~m}, 1 \mathrm{H}), 7.49(\mathrm{~d}, J=7.0 \mathrm{~Hz}, 1 \mathrm{H}) .{ }^{19} \mathrm{~F} \mathrm{NMR}\left(282 \mathrm{MHz}, \mathrm{CDCl}_{3}\right) \delta-$ $75.82\left(\mathrm{~s}, \mathrm{CF}_{3}\right),-123.41--125.51(\mathrm{~m}, \mathrm{CF}) .{ }^{13} \mathrm{C} \mathrm{NMR}\left(75.5 \mathrm{MHz}, \mathrm{CDCl}_{3}\right) \delta 27.62\left(\mathrm{~d},{ }^{3} J_{C F}\right.$ $\left.=7.2 \mathrm{~Hz}, \mathrm{CH}_{2}\right), 46.82(\mathrm{CH}), 99.44\left(\mathrm{~d},{ }^{3} J_{C F}=17.1 \mathrm{~Hz}, \mathrm{CH}\right), 115.66\left(\mathrm{q}, J=288.1 \mathrm{~Hz}, \mathrm{CF}_{3}\right)$, $121.57\left(\mathrm{~d},{ }^{4} J_{C F}=4.4 \mathrm{~Hz}, \mathrm{CH}\right), 127.58\left(\mathrm{~d},{ }^{4} J_{C F}=3.8 \mathrm{~Hz}, \mathrm{CH}\right), 128.04\left(\mathrm{~d},{ }^{2} J_{C F}=26.2 \mathrm{~Hz}\right.$, C), $129.13(\mathrm{CH}), 129.59(\mathrm{CH}), 133.32\left(\mathrm{~d},{ }^{3} J_{C F}=6.3 \mathrm{~Hz}, \mathrm{C}\right), 156.14\left(\mathrm{~d},{ }^{1} J_{C F}=252.8 \mathrm{~Hz}\right.$, C), 156.35 (q, $\left.{ }^{2} J_{C F}=37.3 \mathrm{~Hz}, \mathrm{C}\right)$. HRMS (EI) calcd for $\mathrm{C}_{12} \mathrm{H}_{9} \mathrm{~F}_{4} \mathrm{NO}[\mathrm{M}+\mathrm{H}]^{+}: 260.0620$, found: 260.0622. The enantiomeric excess was determined with the aid of HPLC analysis: Chiralpak IC (25 cm x 0.46 cm column), hexane:isopropanol 99.8:0.2 as eluent and flow $=1.5 \mathrm{~mL} / \mathrm{min}: t_{\mathrm{R} \text { major }}=8.8 \mathrm{~min}, t_{\mathrm{R} \text { minor }}=9.7 \mathrm{~min}$.

(S)-N-(4,8-Difluoro-1,2-dihydronaphthalen-1-yl)-2,2,2-trifluoroacetamide (12c).<smiles>O=C(N[C@H]1CC=C(F)c2cccc(F)c21)C(F)(F)F</smiles>

Following the general procedure, 12c was obtained as a white solid (38 $\mathrm{mg}, 92 \%)$. $[\alpha]_{\mathrm{D}}{ }^{25}$ $=-112.8\left(\mathrm{c} 1.0 ; \mathrm{CHCl}_{3}\right) . \mathrm{Mp} 105-107{ }^{\circ} \mathrm{C} .{ }^{1} \mathrm{H}$ NMR $\left(300 \mathrm{MHz}, \mathrm{CDCl}_{3}\right) \delta 2.71-2.77(\mathrm{~m}$, 2H), $5.46-5.57(\mathrm{~m}, 2 \mathrm{H}), 6.53(\mathrm{~s}, 1 \mathrm{H}), 7.05-7.16(\mathrm{~m}, 1 \mathrm{H}), 7.31(\mathrm{~d}, J=7.5 \mathrm{~Hz}, 1 \mathrm{H}), 7.37$ $-7.48(\mathrm{~m}, 1 \mathrm{H}) .{ }^{19} \mathrm{~F} \mathrm{NMR}\left(282 \mathrm{MHz}, \mathrm{CDCl}_{3}\right) \delta-75.79\left(\mathrm{~s}, \mathrm{CF}_{3}\right),-116.76(\mathrm{td}, J=9.2,5.5$ $\mathrm{Hz}, \mathrm{CF}),-124.03(\mathrm{ddd}, J=14.0,9.7,5.0 \mathrm{~Hz}, \mathrm{CF}) .{ }^{13} \mathrm{C} \mathrm{NMR}\left(75.5 \mathrm{MHz}, \mathrm{CDCl}_{3}\right) \delta 27.5$ $\left(\mathrm{d},{ }^{3} J_{C F}=6.9 \mathrm{~Hz}, \mathrm{CH}_{2}\right), 39.5\left(\mathrm{~d},{ }^{4} J_{C F}=2.6 \mathrm{~Hz}, \mathrm{CH}\right), 100.9\left(\mathrm{~d},{ }^{2} J_{C F}=16.5 \mathrm{~Hz}, \mathrm{CH}\right), 115.5$ $\left(\mathrm{q},{ }^{1} J_{C F}=285.6 \mathrm{~Hz}, \mathrm{C}\right), 116.7\left(\mathrm{~d},{ }^{2} J_{C F}=21.7 \mathrm{~Hz}, \mathrm{CH}\right), 117.4\left(\mathrm{dd},{ }^{3} J_{C F}=4.6,{ }^{4} J_{C F}=3.5\right.$ $\mathrm{Hz}, \mathrm{CH}), 119.5\left(\mathrm{dd},{ }^{2} J_{C F}=17.6,{ }^{3} J_{C F}=6.2 \mathrm{~Hz}, \mathrm{C}\right), 130.2\left(\mathrm{dd},{ }^{2} J_{C F}=27.3,{ }^{3} J_{C F}=4.0 \mathrm{~Hz}\right.$, C), $130.9\left(\mathrm{~d},{ }^{3} J_{C F}=8.6 \mathrm{~Hz}, \mathrm{CH}\right), 155.2\left(\mathrm{dd},{ }^{1} J_{C F}=251.9,{ }^{4} J_{C F}=4.8 \mathrm{~Hz}, \mathrm{C}\right), 156.1\left(\mathrm{q},{ }^{2} J_{\mathrm{CF}}\right.$ $=37.5 \mathrm{~Hz}, \mathrm{C}), 159.6\left(\mathrm{dd},{ }^{1} J_{C F}=250.0,{ }^{4} J_{C F}=5.2 \mathrm{~Hz}, \mathrm{C}\right)$. HRMS (EI) calcd for $\mathrm{C}_{12} \mathrm{H}_{8} \mathrm{~F}_{5} \mathrm{NO}$ $\left[\mathrm{M}+\mathrm{NH}_{4}\right]^{+}: 295.0864$, found: 295.0864 .

(S)-2,2,2-Trifluoro- $N$-[4-fluoro-7-(trifluoromethyl)-1,2-dihydronaphthalen-1yl]acetamide (12d) 
<smiles>O=C(NC1CC=C(F)c2ccc(C(F)(F)F)cc21)C(F)(F)F</smiles>

Following the general procedure, 12d was obtained as a white solid (38 $\mathrm{mg}, 98 \%)$. $[\alpha]_{\mathrm{D}}{ }^{25}$ $=-107.8\left(\mathrm{c} 1.0 ; \mathrm{CHCl}_{3}\right) . \mathrm{Mp} 140-142{ }^{\circ} \mathrm{C} .{ }^{1} \mathrm{H} \mathrm{NMR}\left(300 \mathrm{MHz}, \mathrm{CDCl}_{3}\right) \delta 2.65(\mathrm{ddd}, J=$ $17.7,9.9,5.2 \mathrm{~Hz}, 1 \mathrm{H}), 2.82(\mathrm{dtd}, J=17.5,6.0,3.7 \mathrm{~Hz}, 1 \mathrm{H}), 5.28(\mathrm{dt}, J=8.5,5.6 \mathrm{~Hz}, 1 \mathrm{H})$, $5.62(\mathrm{ddd}, J=12.5,5.6,3.7 \mathrm{~Hz}, 1 \mathrm{H}), 6.76(\mathrm{~d}, J=6.3 \mathrm{~Hz}, 1 \mathrm{H}), 7.57-7.63(\mathrm{~m}, 2 \mathrm{H}), 7.67$ $(\mathrm{dd}, J=8.1,0.9 \mathrm{~Hz}, 1 \mathrm{H}) .{ }^{19} \mathrm{~F} \mathrm{NMR}\left(282 \mathrm{MHz}, \mathrm{CDCl}_{3}\right) \delta-62.82\left(\mathrm{~s}, \mathrm{CF}_{3}\right),-75.75\left(\mathrm{~s}, \mathrm{CF}_{3}\right)$, $-124.26--124.40(\mathrm{~m}, \mathrm{CF}) .{ }^{13} \mathrm{C} \mathrm{NMR}\left(75.5 \mathrm{MHz}, \mathrm{CDCl}_{3}\right) \delta 27.51\left(\mathrm{~d},{ }^{3} J_{C F}=6.8 \mathrm{~Hz}, \mathrm{CH}_{2}\right)$. $46.39(\mathrm{CH}), 102.17\left(\mathrm{~d},{ }^{2} J_{C F}=16.8 \mathrm{~Hz}, \mathrm{CH}\right), 115.56\left(\mathrm{q},{ }^{1} J_{C F}=287.8 \mathrm{~Hz}, \mathrm{C}\right), 122.06(\mathrm{~d}$, $\left.{ }^{3} J_{C F}=4.3 \mathrm{~Hz}, \mathrm{CH}\right), 123.48\left(\mathrm{q},{ }^{1} J_{C F}=272.3 \mathrm{~Hz}\right), 124.44\left(\mathrm{dd},{ }^{3} J_{C F}=3.5 \mathrm{~Hz},{ }^{4} J_{C F}=3.5 \mathrm{~Hz}\right.$, $\mathrm{CH}), 126.19\left(\mathrm{~d},{ }^{3} J_{C F}=3.5 \mathrm{~Hz}, \mathrm{CH}\right), 131.25\left(\mathrm{~d},{ }^{2} J_{C F}=26.5 \mathrm{~Hz}, \mathrm{C}\right), 131.44\left(\mathrm{q},{ }^{2} J_{C F}=33.0\right.$ $\mathrm{Hz}, \mathrm{C}), 133.95\left(\mathrm{~d},{ }^{3} J_{C F}=6.0 \mathrm{~Hz}, \mathrm{C}\right), 155.19\left(\mathrm{~d},{ }^{1} J_{C F}=253.4 \mathrm{~Hz}, \mathrm{C}\right), 156.55\left(\mathrm{q},{ }^{2} J_{C F}=\right.$ $37.5 \mathrm{~Hz}, \mathrm{C})$. HRMS (EI) calcd for $\mathrm{C}_{13} \mathrm{H}_{8} \mathrm{~F}_{7} \mathrm{NO}\left[\mathrm{M}+\mathrm{NH}_{4}\right]^{+}: 345.0838$, found: 345.0838 .

(S)-N-(7-Chloro-4-fluoro-1,2-dihydronaphthalen-1-yl)-2,2,2-trifluoroacetamide (12e)<smiles>O=C(NC1CC=C(F)c2ccc(Cl)cc21)C(F)(F)F</smiles>

Following the general procedure, $\mathbf{1 2 f}$ was obtained as a white solid $(27 \mathrm{mg}, 96 \%)$. $[\alpha]_{\mathrm{D}}{ }^{25}$ $=-52.9\left(\mathrm{c} 1.0 ; \mathrm{CHCl}_{3}\right) . \mathrm{Mp} 95-97{ }^{\circ} \mathrm{C} .{ }^{1} \mathrm{H}$ NMR $\left(300 \mathrm{MHz}, \mathrm{CDCl}_{3}\right) \delta 2.59$ (ddd, $J=17.5$, 9.9, $5.1 \mathrm{~Hz}, 1 \mathrm{H}), 2.78(\mathrm{dtd}, J=17.4,5.9,3.6 \mathrm{~Hz}, 1 \mathrm{H}), 5.20(\mathrm{dt}, J=8.5,5.6 \mathrm{~Hz}, 1 \mathrm{H}), 5.48$ (ddd, $J=12.7,5.6,3.6 \mathrm{~Hz}, 1 \mathrm{H}), 6.69$ (d, $J=4.0 \mathrm{~Hz}, 1 \mathrm{H}), 7.31-7.40$ (m, 2H), 7.42 (d, $J$ $=8.2 \mathrm{~Hz}, 1 \mathrm{H}) .{ }^{19} \mathrm{~F}$ NMR $\left(282 \mathrm{MHz}, \mathrm{CDCl}_{3}\right) \delta-75.72\left(\mathrm{~s}, \mathrm{CF}_{3}\right),-124.24--124.41(\mathrm{~m}$, $\mathrm{CF}) .{ }^{13} \mathrm{C} \mathrm{NMR}\left(75.5 \mathrm{MHz}, \mathrm{CDCl}_{3}\right) \delta 27.6\left(\mathrm{~d},{ }^{3} J_{\mathrm{CF}}=7.0 \mathrm{~Hz}, \mathrm{CH}_{2}\right), 46.5(\mathrm{CH}), 99.8\left(\mathrm{~d},{ }^{2} J_{C F}\right.$ $=17.0 \mathrm{~Hz}), 115.6\left(\mathrm{q},{ }^{1} J_{C F}=288.0 \mathrm{~Hz}, \mathrm{C}\right), 123.0\left(\mathrm{~d},{ }^{3} J_{C F}=4.1 \mathrm{~Hz}, \mathrm{CH}\right), 126.5\left(\mathrm{~d},{ }^{2} J_{C F}=\right.$ $26.7 \mathrm{~Hz}, \mathrm{C}), 127.8\left(\mathrm{~d},{ }^{4} J_{C F}=3.5 \mathrm{~Hz}, \mathrm{CH}\right), 129.3(\mathrm{CH}), 135.1\left(\mathrm{~d},{ }^{3} J_{C F}=6.2 \mathrm{~Hz}, \mathrm{C}\right), 135.2$ (C), $155.6\left(\mathrm{~d},{ }^{1} J_{C F}=253.0 \mathrm{~Hz}, \mathrm{C}\right), 156.4\left(\mathrm{~d},{ }^{2} J_{C F}=37.6 \mathrm{~Hz}, \mathrm{C}\right)$. HRMS (EI) calcd for $\mathrm{C}_{12} \mathrm{H}_{8} \mathrm{~F}_{4} \mathrm{NOCl}[\mathrm{M}+\mathrm{H}]^{+}:$294.0230, found: 294.0231. The enantiomeric excess was 
determined with the aid of HPLC analysis: Chiralpak IC ( $25 \mathrm{~cm} \times 0.46 \mathrm{~cm}$ column), hexane:isopropanol 99.8:0.2 as eluent and flow $=1.5 \mathrm{~mL} / \mathrm{min}: t_{\mathrm{R} \text { major }}=10.0 \mathrm{~min}, t_{\mathrm{R}}$ minor $=11.4 \mathrm{~min}$.

\section{(S)-2,2,2-Trifluoro- $N$-(4-fluoro-7-methyl-1,2-dihydronaphthalen-1-yl)acetamide} (12f)<smiles>Cc1ccc2c(c1)C(NC(=O)C(F)(F)F)CC=C2F</smiles>

Following the general procedure, 12e was obtained as a white solid $(25 \mathrm{mg}, 69 \%)$. $[\alpha]_{\mathrm{D}}{ }^{25}$ $=-136.4\left(\mathrm{c} 1.0 ; \mathrm{CHCl}_{3}\right) . \mathrm{Mp} 110-112{ }^{\circ} \mathrm{C} .{ }^{1} \mathrm{H} \mathrm{NMR}\left(300 \mathrm{MHz}, \mathrm{CDCl}_{3}\right) \delta 2.37(\mathrm{~s}, 3 \mathrm{H})$, $2.58(\mathrm{ddt}, J=17.4,5.8,4.3 \mathrm{~Hz}, 1 \mathrm{H}), 2.76$ (dtd, $J=17.3,5.9,3.4 \mathrm{~Hz}, 1 \mathrm{H}), 5.12-5.21$ (m, 1H), 5.39 (ddd, $J=12.9,5.8,3.3 \mathrm{~Hz}, 1 \mathrm{H}), 6.65(\mathrm{~d}, J=2.9 \mathrm{~Hz}, 1 \mathrm{H}), 7.15(\mathrm{~s}, 1 \mathrm{H}), 7.21$ (dd, $J=7.9,0.8 \mathrm{~Hz}, 1 \mathrm{H}), 7.38(\mathrm{~d}, J=7.8 \mathrm{~Hz}, 1 \mathrm{H}) .{ }^{19} \mathrm{~F} \mathrm{NMR}\left(282 \mathrm{MHz}, \mathrm{CDCl}_{3}\right) \delta-75.79(\mathrm{~s}$, $\left.\mathrm{CF}_{3}\right),-124.44--124.80(\mathrm{~m}, \mathrm{CF}) .{ }^{13} \mathrm{C} \mathrm{NMR}\left(75.5 \mathrm{MHz}, \mathrm{CDCl}_{3}\right) \delta 21.4\left(\mathrm{CH}_{3}\right), 27.7(\mathrm{~d}$, $\left.{ }^{3} J_{C F}=7.2 \mathrm{~Hz}, \mathrm{CH}_{2}\right), 46.9(\mathrm{CH}), 98.2\left(\mathrm{~d},{ }^{2} J_{C F}=17.0 \mathrm{~Hz}, \mathrm{CH}\right), 115.7\left(\mathrm{q},{ }^{1} J_{C F}=288.1 \mathrm{~Hz}\right.$, C), $121.6\left(\mathrm{~d},{ }^{3} J_{C F}=4.0 \mathrm{~Hz}, \mathrm{CH}\right), 125.3\left(\mathrm{~d},{ }^{2} J_{C F}=26.3 \mathrm{~Hz}, \mathrm{C}\right), 128.4\left(\mathrm{~d},{ }^{4} J_{C F}=3.7 \mathrm{~Hz}\right.$, $\mathrm{CH}), 129.7(\mathrm{CH}), 133.3\left(\mathrm{~d},{ }^{3} J_{C F}=6.4 \mathrm{~Hz}, \mathrm{C}\right), 139.9$ (C), 156.3 (q, $\left.{ }^{2} J_{C F}=37.3 \mathrm{~Hz}, \mathrm{C}\right)$, $156.4\left(\mathrm{~d},{ }^{1} J_{C F}=252.6 \mathrm{~Hz}, \mathrm{C}\right)$. HRMS (EI) calcd for $\mathrm{C}_{13} \mathrm{H}_{11} \mathrm{~F}_{4} \mathrm{NO}[\mathrm{M}+\mathrm{H}]^{+}: 274.0847$, found: 274.0850 . 
General procedure of synthesis of fluoroalkenes $12 \mathrm{~b}$ and $12 \mathrm{~g}$.<smiles>CC(C)(C)[S@](=O)N[C@H]1CC=Cc2ccc(F)cc21</smiles>

$14 b$<smiles>CC(C)(C)[S@](=O)N[C@H]1CC=Cc2c1ccc1ccccc21</smiles>

$14 \mathrm{~g}$
1. $\mathrm{HCl}, \mathrm{MeOH}, 0^{\circ} \mathrm{C}$

2. HF/py, NBS, DCM, $0^{\circ} \mathrm{C}$ a rt, $12 \mathrm{~h}$.

3. $\left(\mathrm{CF}_{3} \mathrm{CO}\right)_{2} \mathrm{O}, \mathrm{Et}_{3} \mathrm{~N}, \mathrm{DCM}$

4. $\mathrm{NaH}, \mathrm{DMF}, \mathrm{rt}$.

4 consecutive reactions and one purification.<smiles>O=C(N[C@@H]1CC=C(F)c2ccc(F)cc21)C(F)(F)F</smiles><smiles>O=C(N[C@H]1CC=C(F)c2c1ccc1ccccc21)C(F)(F)F</smiles>

$12 \mathrm{~g}$

Hydrogen chloride (10.0 equiv, $4 \mathrm{M}$ solution in 1,4-dioxane) was added at $0^{\circ} \mathrm{C}$ to a solution of the corresponding sulfinyl amine (2.6 mmol, 1.0 equiv) in $\mathrm{MeOH}(2.6 \mathrm{ml}, 1$ M). After 30 minutes the deprotection was complete and the solution was concentrated to dryness. The residue was re-dissolved in $\mathrm{CH}_{2} \mathrm{Cl}_{2}(2.6 \mathrm{ml}, 0.1 \mathrm{M}), 2 \mathrm{M} \mathrm{NaOH}$ was added and extracted three times with dichloromethane. The combined organic layers were dried over anhydrous $\mathrm{Na}_{2} \mathrm{SO}_{4}$ and the solvents removed under reduced pressure.

To a solution of the crude 1-amino-1,2-dihydronaphthalene derivatives (25.0 mmol, 1.0 equiv) in $\mathrm{CH}_{2} \mathrm{Cl}_{2}(0.5 \mathrm{M}), \mathrm{HF} \cdot \mathrm{Py}$ was added at $0^{\circ} \mathrm{C}$ ( 3 equiv), followed by $N$ bromosuccinimide (1.1 equiv). Upon stirring at $0^{\circ} \mathrm{C}$ for $15 \mathrm{~min}$ and then at room temperature overnight, the reaction mixture was poured onto slightly basic ice water adjusted with $\mathrm{NH}_{4} \mathrm{OH}$, extracted with $\mathrm{CH}_{2} \mathrm{Cl}_{2}$ eight times, washed with $\mathrm{NaHCO}_{3}$, dried over $\mathrm{Na}_{2} \mathrm{SO}_{4}$ and concentrated.

Next, the residue was dissolved in anhydrous DCM (0.1M). To the resulting solution, triethylamine ( 2.5 equiv) and trifluoroacetic anhydride (5 equiv) were added at $0^{\circ} \mathrm{C}$. The reaction mixture was stirred at $0^{\circ} \mathrm{C}$ for $15 \mathrm{~min}$ and then at room temperature until TLC revealed the disappearance of the starting material. The reaction mixture was quenched with $\mathrm{H}_{2} \mathrm{O}$ and extracted three times with $\mathrm{CH}_{2} \mathrm{Cl}_{2}$. The combined organic layers were washed with a saturated aqueous solution of $\mathrm{NaHCO}_{3}$, dried over $\mathrm{Na}_{2} \mathrm{SO}_{4}$ and concentrated.

Finally, a solution of the above compound (1.0 equiv) in dry DMF (0.06M) was treated with $\mathrm{NaH}$ (4 equiv) at $0^{\circ} \mathrm{C}$. The reaction mixture was stirred at $0^{\circ} \mathrm{C}$ for $15 \mathrm{~min}$ then at room temperature until TLC revealed the disappearance of the starting material. 
The reaction mixture was quenched with $\mathrm{H}_{2} \mathrm{O}$, extracted three times with $\mathrm{Et}_{2} \mathrm{O}$, and the combined organic layers were washed with $\mathrm{H}_{2} \mathrm{O}$ three times and dried over $\mathrm{Na}_{2} \mathrm{SO}_{4}$. Flash chromatography afforded the desired compound.

(Note: a splitting or broadening of some peaks was observed due to rotamers).

(S)-N-(4,7-Difluoro-1,2-dihydronaphthalen-1-yl)-2,2,2-trifluoroacetamide (12b)<smiles>O=C(NC1CC=C(F)c2ccc(F)cc21)C(F)(F)F</smiles>

Following the general procedure, $\mathbf{1 2 b}$ was obtained as a colorless oil $(5 \mathrm{mg}, 66 \%)$. $[\alpha]_{\mathrm{D}}{ }^{25}$ $=-91.2\left(\mathrm{c} 1.0 ; \mathrm{CHCl}_{3}\right) .{ }^{1} \mathrm{H} \mathrm{NMR}\left(300 \mathrm{MHz}, \mathrm{CDCl}_{3}\right) \delta 2.54-2.67(\mathrm{~m}, 2 \mathrm{H}), 2.75-2.87$ (m, 1H), $5.23(\mathrm{dt}, J=8.6,5.6 \mathrm{~Hz}, 1 \mathrm{H}), 5.46(\mathrm{ddd}, J=12.8,5.6,3.5 \mathrm{~Hz}, 1 \mathrm{H}), 6.64(\mathrm{~d}, J=$ $8.6 \mathrm{~Hz}, 1 \mathrm{H}), 7.07-7.16(\mathrm{~m}, 2 \mathrm{H}), 7.46-7.55(\mathrm{~m}, 1 \mathrm{H}) .{ }^{19} \mathrm{~F} \mathrm{NMR}\left(282 \mathrm{MHz}, \mathrm{CDCl}_{3}\right) \delta$ $75.76\left(\mathrm{~s}, \mathrm{CF}_{3}\right),-109.58$ (d, $\left.J=7.8 \mathrm{~Hz}, \mathrm{CF}\right),-123.96(\mathrm{~d}, J=12.1 \mathrm{~Hz}, \mathrm{CF}) .{ }^{13} \mathrm{C}$ NMR $(75.5$

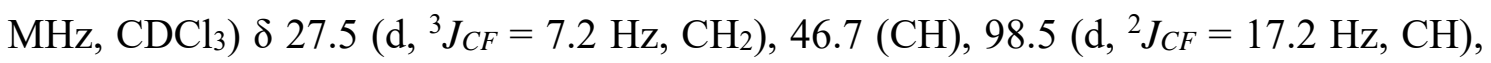
$115.0\left(\mathrm{dd},{ }^{2} J_{C F}=23.2,{ }^{4} J_{C F}=3.3 \mathrm{~Hz}, \mathrm{CH}\right), 115.6\left(\mathrm{q},{ }^{1} J_{C F}=288.1 \mathrm{~Hz}, \mathrm{C}\right), 115.9\left(\mathrm{~d},{ }^{2} J_{C F}\right.$ $=21.9 \mathrm{~Hz}, \mathrm{CH}), 123.7\left(\mathrm{dd},{ }^{3} J_{C F}=8.3,{ }^{3} J_{C F}=4.3 \mathrm{~Hz}, \mathrm{CH}\right), 124.2\left(\mathrm{dd},{ }^{2} J_{C F}=26.7,{ }^{4} J_{C F}=\right.$ $3.0 \mathrm{~Hz}, \mathrm{C}), 135.9\left(\mathrm{dd},{ }^{3} J_{C F}=7.0 \mathrm{~Hz},{ }^{3} J_{C F}=7.0 \mathrm{~Hz}, \mathrm{C}\right), 155.7\left(\mathrm{~d},{ }^{1} J_{C F}=252.8 \mathrm{~Hz}, \mathrm{C}\right)$, $156.4\left(\mathrm{q},{ }^{2} J_{C F}=37.3 \mathrm{~Hz}, \mathrm{C}\right), 163.0\left(\mathrm{~d},{ }^{1} J_{C F}=251.0 \mathrm{~Hz}, \mathrm{C}\right)$. HRMS (EI) calcd for $\mathrm{C}_{12} \mathrm{H}_{8} \mathrm{NOF}_{5}[\mathrm{M}+\mathrm{H}]^{+}: 278.0536$, found: 278.0536 .

(S)-2,2,2-Trifluoro- $N$-(4-fluoro-1,2-dihydrophenanthren-1-yl)acetamide (12g)<smiles>O=C(NC1CC=C(F)c2c1ccc1ccccc21)C(F)(F)F</smiles>

Following the general procedure, $\mathbf{1 2 g}$ was obtained as a white solid (30 $\mathrm{mg}, 40 \%)$. $[\alpha]_{\mathrm{D}}{ }^{25}$ $=-146.1$ (c 1.0; $\left.\mathrm{CHCl}_{3}\right)$. Mp 126-128 ${ }^{\circ} \mathrm{C} .{ }^{1} \mathrm{H} \mathrm{NMR}\left(300 \mathrm{MHz}, \mathrm{CDCl}_{3}\right) \delta 2.69$ (ddt, $J=$ 17.5, 6.9, $3.5 \mathrm{~Hz}, 1 \mathrm{H}), 2.85(\mathrm{dtd}, J=17.3,5.8,3.3 \mathrm{~Hz}, 1 \mathrm{H}), 5.27-5.34(\mathrm{~m}, 1 \mathrm{H}), 5.71$ (ddd, $J=15.5,6.5,3.2 \mathrm{~Hz}, 1 \mathrm{H}), 6.81(\mathrm{~d}, J=7.7 \mathrm{~Hz}, 1 \mathrm{H}), 7.48$ (dd, $J=8.4,2.0 \mathrm{~Hz}, 1 \mathrm{H})$, $7.54-7.63(\mathrm{~m}, 2 \mathrm{H}), 7.83-7.92(\mathrm{~m}, 2 \mathrm{H}), 8.57-8.64(\mathrm{~m}, 1 \mathrm{H}) .{ }^{19} \mathrm{~F}$ NMR $(282 \mathrm{MHz}$, $\left.\mathrm{CDCl}_{3}\right) \delta-75.76\left(\mathrm{~s}, \mathrm{CF}_{3}\right),-111.85(\mathrm{~d}, J=15.5 \mathrm{~Hz}, \mathrm{CF}) .{ }^{13} \mathrm{C} \mathrm{NMR}\left(75.5 \mathrm{MHz}, \mathrm{CDCl}_{3}\right) \delta$ 
$27.2\left(\mathrm{~d},{ }^{3} J_{C F}=7.4 \mathrm{~Hz}, \mathrm{CH}_{2}\right), 47.9(\mathrm{CH}), 101.6\left(\mathrm{~d},{ }^{2} J_{C F}=20.4 \mathrm{~Hz}\right), 115.6\left(\mathrm{q},{ }^{1} J_{C F}=288.4\right.$ $\left.\mathrm{Hz}, \mathrm{CF}_{3}\right), 125.4\left(\mathrm{~d},{ }^{4} J_{C F}=3.4 \mathrm{~Hz}, \mathrm{CH}\right), 126.6(\mathrm{CH}), 127.4(\mathrm{CH}), 128.0\left(\mathrm{~d},{ }^{2} J_{C F}=19.3\right.$ $\mathrm{Hz}, \mathrm{C}), 128.7(\mathrm{CH}), 128.8(\mathrm{CH}), 130.8(\mathrm{CH}), 131.8$ (d, $\left.{ }^{3} J_{C F}=4.5 \mathrm{~Hz}, \mathrm{C}\right), 132.9\left(\mathrm{~d},{ }^{3} J_{C F}\right.$ $=5.6 \mathrm{~Hz}, \mathrm{C}), 134.3(\mathrm{C}), 156.4\left(\mathrm{q},{ }^{2} J_{C F}=37.3 \mathrm{~Hz}, \mathrm{C}\right), 159.2\left(\mathrm{~d},{ }^{1} J_{C F}=255.3 \mathrm{~Hz}, \mathrm{CF}\right)$. HRMS (EI) calcd for $\mathrm{C}_{16} \mathrm{H}_{11} \mathrm{NOF}_{4}[\mathrm{M}+\mathrm{H}]^{+}: 310.0850$, found: 310.0833 .

\section{General procedure of synthesis of aminoalcohols 15a,c,d.}

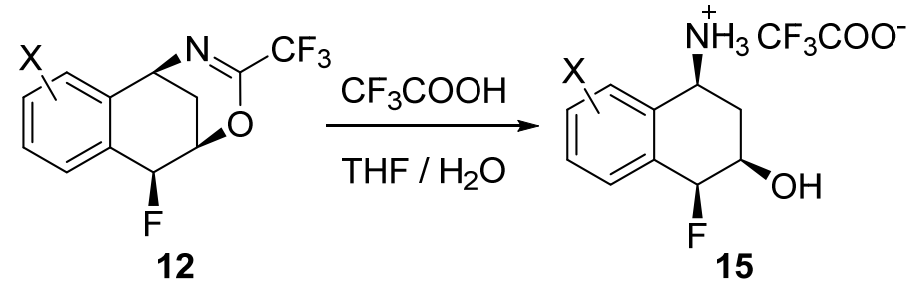

To a solution of compound 12 in $\mathrm{THF} / \mathrm{H}_{2} \mathrm{O}(3: 1,0.01 \mathrm{M})$, trifluoroacetic acid (one drop) was added at room temperature. The reaction mixture was stirred at room temperature until TLC revealed the disappearance of the starting material. The solvents were then removed under pressure, affording the pure amino alcohol derivatives $\mathbf{1 5}$ without the need for further purification.

(The signals corresponding to the TFA counteranion are only observed in some ${ }^{13} \mathrm{C} N \mathrm{NR}$ spectra).

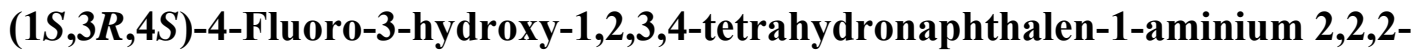
trifluoroacetate (15a)<smiles>[NH3+][C@H]1C[C@H](O)[C@H](F)c2ccccc21</smiles>

Following the general procedure, 15a was obtained as a brown oil $(28 \mathrm{mg},>99 \%$, dr $<20: 1) .[\alpha]_{\mathrm{D}}{ }^{25}=-26.2\left(\mathrm{c} 1.0 ; \mathrm{CHCl}_{3}\right) .{ }^{1} \mathrm{H}$ NMR $(300 \mathrm{MHz}, \mathrm{MeOD}) \delta 2.32-2.39(\mathrm{~m}, 2 \mathrm{H})$, $4.34(\mathrm{dtd}, J=16.9,5.2,3.2 \mathrm{~Hz}, 1 \mathrm{H}), 4.61(\mathrm{td}, J=5.7,2.6 \mathrm{~Hz}, 1 \mathrm{H}), 5.55(\mathrm{dd}, J=50.9,3.1$ $\mathrm{Hz}, 1 \mathrm{H}), 7.45-7.62(\mathrm{~m}, 4 \mathrm{H}) .{ }^{19} \mathrm{~F}$ NMR (282 MHz, MeOD) $\delta-77.03$ (s, CF $\left.3 \mathrm{COO}\right)$, $185.25(\mathrm{dd}, J=50.8,13.5 \mathrm{~Hz}, \mathrm{CHF}) .{ }^{13} \mathrm{C} \mathrm{NMR}(75.5 \mathrm{MHz}, \mathrm{MeOD}) \delta 29.7\left(\mathrm{~d},{ }^{3} J_{C F}=5.3\right.$ $\left.\mathrm{Hz}, \mathrm{CH}_{2}\right), 47.9(\mathrm{CH}), 65.6\left(\mathrm{~d},{ }^{2} J_{C F}=18.3 \mathrm{~Hz}, \mathrm{CH}\right), 89.5\left(\mathrm{~d},{ }^{1} J_{C F}=176.4 \mathrm{~Hz}, \mathrm{CH}\right), 127.6$ $(\mathrm{CH}), 129.1(\mathrm{CH}), 129.2(\mathrm{CH}), 129.4(\mathrm{CH}), 131.5\left(\mathrm{~d},{ }^{3} J_{C F}=3.6 \mathrm{~Hz}, \mathrm{C}\right), 133.5\left(\mathrm{~d},{ }^{2} J_{C F}=\right.$ 
18.7 Hz, C). HRMS (EI) calcd for $\mathrm{C}_{10} \mathrm{H}_{12} \mathrm{NOF} \quad\left[\mathrm{M}+\mathrm{H}-\mathrm{CF}_{3} \mathrm{COO}\right]^{+}: 182.0970$, found: 182.0976 .

$(1 S, 3 R, 4 S)-4,8$-Difluoro-3-hydroxy-1,2,3,4-tetrahydronaphthalen-1-aminium 2,2,2trifluoroacetate (15c)<smiles>[NH3+][C@H]1C[C@H](O)[C@H](F)c2cccc(F)c21</smiles>

Following the general procedure, 15c was obtained as a brown oil $(30 \mathrm{mg},>99 \%$, $\mathrm{dr}$ $<20: 1) .[\alpha]_{\mathrm{D}}{ }^{25}=-90.3$ (c 1.0; Acetona). ${ }^{1} \mathrm{H}$ NMR (300 MHz, MeOD) $\delta 2.35$ (ddd, $J=15.1$, 5.7, $2.1 \mathrm{~Hz}, 1 \mathrm{H}), 2.47$ (dddd, $J=14.3,6.3,4.5,3.5 \mathrm{~Hz}, 1 \mathrm{H}), 4.43-4.53$ (m, 1H), $4.76-$ $4.82(\mathrm{~m}, 1 \mathrm{H}), 5.59(\mathrm{dd}, J=49.3,3.2 \mathrm{~Hz}, 1 \mathrm{H}), 7.26(\mathrm{dd}, J=9.6,8.8 \mathrm{~Hz}, 1 \mathrm{H}), 7.47$ (d, $J=$ $7.8 \mathrm{~Hz}, 1 \mathrm{H}), 7.58(\mathrm{td}, J=8.0,5.7 \mathrm{~Hz}, 1 \mathrm{H}) .{ }^{19} \mathrm{~F}$ NMR (282 MHz, MeOD) $\delta-77.28(\mathrm{~s}$, $\left.\mathrm{CF}_{3} \mathrm{COO}\right),-117.56--117.67$ (m, CF), -189.67 - -190.11 (m, CHF). ${ }^{13} \mathrm{C}$ NMR (75.5 MHz, MeOD) $\delta 29.2\left(\mathrm{~d},{ }^{3} J_{C F}=6.6 \mathrm{~Hz}, \mathrm{CH}_{2}\right), 42.6(\mathrm{CH}), 64.9\left(\mathrm{~d},{ }^{2} J_{C F}=17.8 \mathrm{~Hz}, \mathrm{CH}\right), 89.0(\mathrm{dd}$, $\left.{ }^{1} J_{C F}=178.7,{ }^{4} J_{C F}=2.8 \mathrm{~Hz}, \mathrm{CH}\right), 114.6\left(\mathrm{q},{ }^{1} J_{C F}=284.5 \mathrm{~Hz}, \mathrm{CF}_{3}\right.$ del trifluoroacetato $)$, $115.1\left(\mathrm{~d},{ }^{2} J_{C F}=21.2 \mathrm{~Hz}, \mathrm{CH}\right), 118.8\left(\mathrm{dd},{ }^{2} J_{C F}=16.2,{ }^{3} J_{C F}=3.8 \mathrm{~Hz}, \mathrm{C}\right), 123.9(\mathrm{dd}, J=$ 6.6, 3.1 Hz, CH), $131.4(\mathrm{~d}, J=9.0 \mathrm{~Hz}, \mathrm{CH}), 136.3\left(\mathrm{dd},{ }^{2} J_{C F}=19.9,{ }^{3} J_{C F}=2.8 \mathrm{~Hz}, \mathrm{C}\right)$, $157.6\left(\mathrm{~d},{ }^{2} J_{C F}=41.6 \mathrm{~Hz}, \mathrm{COCF}_{3}\right.$ del trifluoroacetato), $160.4\left(\mathrm{~d},{ }^{1} J_{C F}=246.5 \mathrm{~Hz}, \mathrm{C}\right)$. HRMS (EI) calcd for $\mathrm{C}_{10} \mathrm{H}_{11} \mathrm{NOF}_{2}$ [M+H-CF $\mathrm{COO}^{+}: 200.0881$, found: 200.0872 .

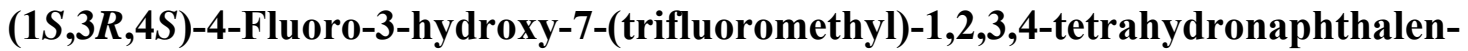
1-aminium 2,2,2-trifluoroacetate (15d)<smiles>N[C@H]1CC(O)[C@H](F)c2ccc(C(F)(F)F)cc21</smiles>

Following the general procedure, 15d was obtained as a brown oil $(10 \mathrm{mg},>99 \%$, $\mathrm{dr}$ $<20: 1) .[\alpha]_{\mathrm{D}}{ }^{25}=-4.1$ (c 1.0; Acetona). ${ }^{1} \mathrm{H}$ NMR (300 MHz, MeOD) $\delta 2.39-2.46(\mathrm{~m}, 1 \mathrm{H})$, $4.41-4.55$ (m, 1H), 4.70 (t, $J=4.5 \mathrm{~Hz}, 1 \mathrm{H}), 5.64$ (dd, $J=49.3,3.0 \mathrm{~Hz}, 1 \mathrm{H}), 7.80-7.87$ (m, 2H), 7.93 (s, 1H). ${ }^{19} \mathrm{~F} \mathrm{NMR}(282 \mathrm{MHz}, \mathrm{MeOD}) \delta-64.29\left(\mathrm{~s}, \mathrm{CF}_{3}\right),-77.25$ (s, $\left.\mathrm{CF}_{3} \mathrm{COO}\right)$, $-189.46(\mathrm{dd}, J=49.2,10.2 \mathrm{~Hz}, \mathrm{CHF}) .{ }^{13} \mathrm{C}$ NMR $(75.5 \mathrm{MHz}, \mathrm{MeOD}) \delta 29.4\left(\mathrm{~d},{ }^{3} J_{C F}=6.3\right.$ $\left.\mathrm{Hz}, \mathrm{CH}_{2}\right), 47.2(\mathrm{CH}), 65.0\left(\mathrm{~d},{ }^{2} J_{\mathrm{CF}}=17.9 \mathrm{~Hz}, \mathrm{CH}\right), 89.1\left(\mathrm{~d},{ }^{1} J_{C F}=178.5 \mathrm{~Hz}, \mathrm{CH}\right), 123.7$ 
$\left(\mathrm{d},{ }^{1} J_{C F}=271.5 \mathrm{~Hz}, \mathrm{C}\right), 125.2\left(\mathrm{~d},{ }^{3} J_{C F}=3.6 \mathrm{~Hz}, \mathrm{CH}\right), 126.1\left(\mathrm{~d},{ }^{3} J_{C F}=3.2 \mathrm{~Hz}, \mathrm{CH}\right), 129.4$

$\left(\mathrm{d},{ }^{3} J_{C F}=6.8 \mathrm{~Hz}, \mathrm{CH}\right), 131.0\left(\mathrm{q},{ }^{2} J_{C F}=32.8 \mathrm{~Hz}, \mathrm{C}\right), 132.4\left(\mathrm{~d},{ }^{3} J_{C F}=3.8 \mathrm{~Hz}, \mathrm{C}\right), 138.2(\mathrm{~d}$, $\left.{ }^{2} J_{C F}=19.2 \mathrm{~Hz}, \mathrm{C}\right)$. HRMS (EI) calcd for $\mathrm{C}_{11} \mathrm{H}_{11} \mathrm{NOF}_{4} \quad\left[\mathrm{M}+\mathrm{H}-\mathrm{CF}_{3} \mathrm{COO}\right]^{+}:$250.0850, found: 250.0844 .

General procedure for the bromofluorination of 2-vinylbenzaldehyde derivatives 16a,e<smiles>[R]c1ccc(C=C)c(C=O)c1</smiles>

$13 a, e$<smiles>[R]c1ccc(C(F)CBr)c(C=O)c1</smiles>

$16 a, e$

To a solution of the corresponding 2-vinylbenzaldehyde derivative in an eppendorf vial in anhydrous DCM $(0.5 \mathrm{M})$ under a nitrogen atmosphere, $N$ bromosuccinimide (NBS) (1.1 equiv) was added at room temperature. After stirring for 5 minutes, the mixture was cooled to $0{ }^{\circ} \mathrm{C}$ and $\mathrm{HF} \cdot \mathrm{Py}$ ( 3 equiv) was added. The reaction mixture was stirred at this temperature until the starting material was consumed ( 1 minute approx.). Then, the reaction was quenched at $0{ }^{\circ} \mathrm{C}$ with aqueous $\mathrm{NH}_{3}(25 \%)$ until gas evolution ceased. The resulting solution was extracted three times with DCM, the combined organic layers washed three times with a saturated aqueous solution of $\mathrm{NaHCO}_{3}$ and dried over anhydrous $\mathrm{Na}_{2} \mathrm{SO}_{4}$. The residue obtained after elimination of the solvent under reduced pressure was purified by flash chromatography using mixtures of hexane:ethyl acetate as eluent.

\section{2-(2-Bromo-1-fluoroetil)benzaldehído (16a).}<smiles>O=Cc1ccccc1C(F)CBr</smiles>

Following the general procedure, 16a was obtained as a colorless oil $(135 \mathrm{mg}, 52 \%) .{ }^{1} \mathrm{H}$ NMR (300 MHz, $\left.\mathrm{CDCl}_{3}\right) \delta 3.61$ (ddd, $\left.J=22.4,11.5,6.5 \mathrm{~Hz}, 1 \mathrm{H}\right), 3.82$ (ddd, $J=25.5$, 11.5, $2.7 \mathrm{~Hz}, 1 \mathrm{H}), 6.51$ (ddd, $J=47.6,6.5,2.6 \mathrm{~Hz}, 1 \mathrm{H}), 7.61$ (td, $J=7.4,1.4 \mathrm{~Hz}, 1 \mathrm{H})$, $7.71(\mathrm{td}, J=7.5,1.4 \mathrm{~Hz}, 1 \mathrm{H}), 7.77(\mathrm{~d}, J=7.7 \mathrm{~Hz}, 1 \mathrm{H}), 7.86(\mathrm{~d}, J=7.5 \mathrm{~Hz}, 1 \mathrm{H}), 10.05$ (s, 1H). ${ }^{19} \mathrm{~F}$ NMR $\left(282 \mathrm{MHz}, \mathrm{CDCl}_{3}\right) \delta-180.73--185.74(\mathrm{~m}, \mathrm{CHF}) .{ }^{13} \mathrm{C}$ NMR $(75.5 \mathrm{MHz}$, 
$\left.\mathrm{CDCl}_{3}\right) \delta 34.6\left(\mathrm{~d},{ }^{2} J_{C F}=25.1 \mathrm{~Hz}, \mathrm{CH}_{2}\right), 89.2\left(\mathrm{~d},{ }^{1} J_{C F}=177.3 \mathrm{~Hz}, \mathrm{CHF}\right), 126.8\left(\mathrm{~d},{ }^{3} J_{C F}=\right.$ $13.9 \mathrm{~Hz}, \mathrm{CH}), 129.1(\mathrm{CH}), 132.0\left(\mathrm{~d},{ }^{3} J_{C F}=3.9 \mathrm{~Hz}, \mathrm{C}\right), 134.2(\mathrm{CH}), 135.4(\mathrm{CH}), 139.0(\mathrm{~d}$, $\left.{ }^{2} J_{C F}=20.3 \mathrm{~Hz}, \mathrm{C}\right), 193.33(\mathrm{CHO})$. HRMS (EI) calcd for $\mathrm{C}_{9} \mathrm{H}_{8} \mathrm{BrFO}[\mathrm{M}+\mathrm{H}]^{+}: 230.9740$, found: 230.9743 .

\section{2-(2-Bromo-1-fluoroethyl)-5-chlorobenzaldehído (16e).}<smiles>O=Cc1cc(Cl)ccc1C(F)CBr</smiles>

Following the general procedure, 16e was obtained as a yellow oil $(160 \mathrm{mg}, 62 \%) .{ }^{1} \mathrm{H}$ NMR (300 MHz, $\left.\mathrm{CDCl}_{3}\right) \delta 3.61$ (ddd, $\left.J=23.8,11.6,6.0 \mathrm{~Hz}, 1 \mathrm{H}\right), 3.82$ (ddd, $J=23.3$, 11.6, $2.9 \mathrm{~Hz}, 1 \mathrm{H}), 6.46$ (ddd, $J=47.2,6.0,2.9 \mathrm{~Hz}, 1 \mathrm{H}), 7.67$ (dd, $J=8.4,2.1 \mathrm{~Hz}, 1 \mathrm{H})$, $7.72(\mathrm{~d}, J=8.4 \mathrm{~Hz}, 1 \mathrm{H}), 7.81-7.84(\mathrm{~m}, 1 \mathrm{H}), 10.01(\mathrm{~s}, 1 \mathrm{H}) .{ }^{19} \mathrm{~F} \mathrm{NMR}\left(282 \mathrm{MHz}, \mathrm{CDCl}_{3}\right)$ $\delta-182.14(\mathrm{dt}, J=47.1,23.5 \mathrm{~Hz}, \mathrm{CHF}) .{ }^{13} \mathrm{C} \mathrm{NMR}\left(75.5 \mathrm{MHz}, \mathrm{CDCl}_{3}\right) \delta 34.3\left(\mathrm{~d},{ }^{2} J_{C F}=\right.$ $\left.25.2 \mathrm{~Hz}, \mathrm{CH}_{2}\right), 88.6\left(\mathrm{~d},{ }^{1} J_{C F}=178.1 \mathrm{~Hz}, \mathrm{CHF}\right), 128.7\left(\mathrm{~d},{ }^{3} J_{C F}=14.0 \mathrm{~Hz}, \mathrm{CH}\right), 133.3(\mathrm{~d}$, $\left.{ }^{3} J_{C F}=3.6 \mathrm{~Hz}, \mathrm{C}\right), 134.1(\mathrm{CH}), 134.7(\mathrm{CH}), 135.4(\mathrm{C}), 137.3\left(\mathrm{~d},{ }^{2} J_{C F}=20.8 \mathrm{~Hz}, \mathrm{C}\right), 191.9$ (COH). HRMS (EI) calcd for $\mathrm{C}_{9} \mathrm{H}_{7} \mathrm{BrClFO}[\mathrm{M}+\mathrm{H}]^{+}: 264.9350$, found: 264.9353 .

\section{Synthesis of 2-fluorovinylbenzaldehyde 17a}

To a solution of 16a in anhydrous THF (0.1M), t-BuOK (2.4 equiv) was added at $0{ }^{\circ} \mathrm{C}$. The reaction was stirred and monitored by TLC analysis. Upon completion, the reaction mixture was quenched with a saturated solution of $\mathrm{NH}_{4} \mathrm{Cl}$ and extracted three times with $\mathrm{Et}_{2} \mathrm{O}$. The combined organic layers were dried over anhydrous $\mathrm{Na}_{2} \mathrm{SO}_{4}$, filtered and concentrated under reduced pressure. The residue was purified by flash chromatography on silica gel (1:1, hexane:ethyl acetate).

\section{2-(1-Fluorovinyl)benzaldehyde (17a)}<smiles>C=C(F)c1ccccc1C=O</smiles> 
${ }^{1} \mathrm{H}$ NMR $\left(300 \mathrm{MHz}, \mathrm{CDCl}_{3}\right) \delta 4.83(\mathrm{dd}, J=47.4,3.5 \mathrm{~Hz}, 1 \mathrm{H}), 5.19$ (dd, $J=16.0,3.4 \mathrm{~Hz}$, 1H), $7.45-7.67(\mathrm{~m}, 3 \mathrm{H}), 7.91-8.04(\mathrm{~m}, 1 \mathrm{H}), 10.32(\mathrm{dd}, J=3.5,0.6 \mathrm{~Hz}, 1 \mathrm{H}) .{ }^{19} \mathrm{~F}$ NMR $\left(282 \mathrm{MHz}, \mathrm{CDCl}_{3}\right) \delta-85.63(\mathrm{ddd}, J=47.4,16.0,2.5 \mathrm{~Hz})$.

\section{Experimental procedure for the synthesis of compounds 18a,e.}

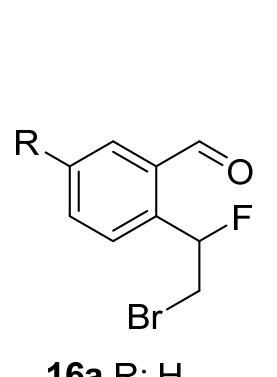

16a R: H

16e $\mathrm{R}: \mathrm{Cl}$
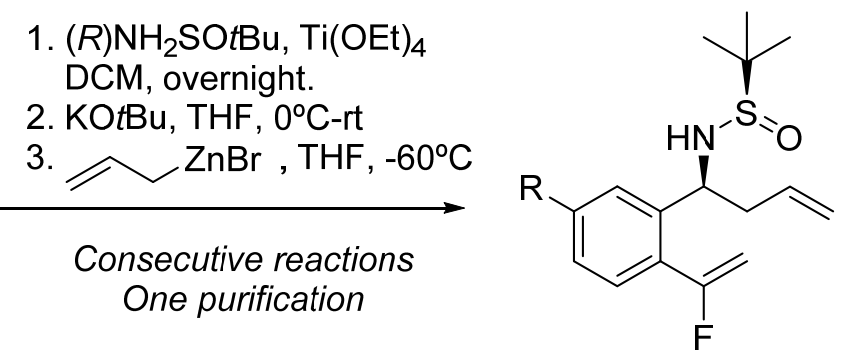

18a R: H, rd >20:1, $50 \%$

18e R: $\mathrm{Cl}$, rd $17: 1,71 \%$

The corresponding aldehyde 16 was dissolved in anhydrous DCM (0.1M) and $\mathrm{Ti}(\mathrm{OEt})_{4}$ (4 equiv) was added. After 5 minutes stirring, the mixture was cooled to $0{ }^{\circ} \mathrm{C}$, (R)-N-terc-butanosulfinamide was added and the mixture was stirred overnight at room temperature. The reaction mixture was then quenched with a saturated aqueous solution of $\mathrm{NaHCO}_{3}$ at $0{ }^{\circ} \mathrm{C}$, forming a white precipitate of titanium oxide. This suspension was filtered through a short pad of Celite ${ }^{\circledR}$ and the filter cake washed with DCM. The clear solution was extracted three times with DCM. The combined organic layers were washed with brine and dried over anhydrous $\mathrm{Na}_{2} \mathrm{SO}_{4}$. The solvent was removed under pressure and the resulting residue was dissolved in THF $(0.1 \mathrm{M})$ and cooled to $0{ }^{\circ} \mathrm{C}$. After 5 minutes, $t$-BuOK ( 1.1 equiv) was added and the mixture was stirred at room temperature until completion, as determined by TLC analysis ( 4 hours approx.). The reaction was then quenched with water and extracted with AcOEt (3 times). The combined organic layers were washed with brine, dried over anhydrous $\mathrm{Na}_{2} \mathrm{SO}_{4}$, and the solvents removed under pressure.

Finally, the residue was dissolved in THF $(0.1 \mathrm{M})$ and cooled to $-40{ }^{\circ} \mathrm{C}$. After stirring for 5 minutes at this temperature, allylzinc bromide (1M in THF, 1.1 equiv) was added and stirring continued for 3 hours. Then, the reaction mixture was quenched at -60 ${ }^{\circ} \mathrm{C}$ with a saturated aqueous solution, extracted 3 times with AcOEt, washed with brine and the combined organic layers dried over anhydrous $\mathrm{Na}_{2} \mathrm{SO}_{4}$. The residue was purified by flash chromatography on silica gel using mixture of hexane:ethyl acetate as eluents. 


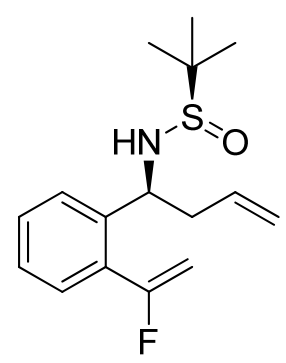

Following the general procedure, 18a was obtained as a yellow oil $(80 \mathrm{mg}, 66 \%$, $\mathrm{dr}$ $>20: 1) .[\alpha]_{\mathrm{D}}{ }^{25}=-135.5\left(\mathrm{c} 1.0 ; \mathrm{CHCl}_{3}\right) .{ }^{1} \mathrm{H} \mathrm{NMR}\left(300 \mathrm{MHz}, \mathrm{CDCl}_{3}\right) \delta 1.17$ (s, 9H), 2.44 (dt, $J=14.1,8.6 \mathrm{~Hz}, 1 \mathrm{H}), 2.65(\mathrm{dt}, J=11.3,5.3 \mathrm{~Hz}, 1 \mathrm{H}), 3.72(\mathrm{~d}, J=2.1 \mathrm{~Hz}, 1 \mathrm{H}), 4.66$ $-4.89(\mathrm{~m}, 2 \mathrm{H}), 5.02(\mathrm{dd}, J=15.9,3.1 \mathrm{~Hz}, 1 \mathrm{H}), 5.12-5.22(\mathrm{~m}, 2 \mathrm{H}), 5.66-5.86(\mathrm{~m}, 1 \mathrm{H})$, $7.23-7.31(\mathrm{~m}, 1 \mathrm{H}), 7.33-7.43(\mathrm{~m}, 2 \mathrm{H}), 7.46(\mathrm{~d}, J=8.0 \mathrm{~Hz}, 1 \mathrm{H}) .{ }^{19} \mathrm{~F}$ NMR $(282 \mathrm{MHz}$, $\left.\mathrm{CDCl}_{3}\right) \delta-84.04(\mathrm{dd}, J=48.2,15.9 \mathrm{~Hz}, \mathrm{CF}) .{ }^{13} \mathrm{C} \mathrm{NMR}\left(75.5 \mathrm{MHz}, \mathrm{CDCl}_{3}\right) \delta 22.5$ $\left(3 \mathrm{xCH}_{3}\right), 43.2\left(\mathrm{CH}_{2}\right), 53.5(\mathrm{CH}), 55.8(\mathrm{C}), 95.2\left(\mathrm{~d},{ }^{2} J_{C F}=22.4 \mathrm{~Hz}, \mathrm{CH}_{2}\right), 119.2\left(\mathrm{CH}_{2}\right)$, $127.3(\mathrm{CH}), 127.5(\mathrm{CH}), 129.8(2 \times \mathrm{xH}), 132.3\left(\mathrm{~d},{ }^{2} J_{\mathrm{CF}}=26.5 \mathrm{~Hz}, \mathrm{C}\right), 134.2(\mathrm{CH}), 140.7$ (C), $163.4\left(\mathrm{~d},{ }^{1} J_{C F}=255.2 \mathrm{~Hz}\right)$. HRMS (EI) calcd for $\mathrm{C}_{16} \mathrm{H}_{22} \mathrm{FNOS}[\mathrm{M}+\mathrm{H}]^{+}:$296.1493, found: 296.1479 .

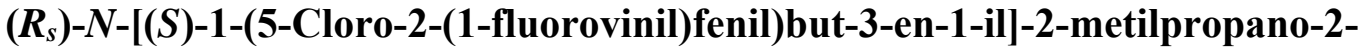 sulfinamida (18e)}<smiles>C=CC[C@H](NS(=O)C(C)(C)C)c1cc(Cl)ccc1C(=C)F</smiles>

Following the general procedure, 18e was obtained as a yellow oil (40 mg, 71\%, dr 17:1). $[\alpha]_{\mathrm{D}}^{25}=-135.5\left(\mathrm{c} 1.0 ; \mathrm{CHCl}_{3}\right) .{ }^{1} \mathrm{H}$ NMR $\left(300 \mathrm{MHz}, \mathrm{CDCl}_{3}\right) \delta 2.39(\mathrm{dd}, J=14.1,8.7 \mathrm{~Hz}$, $1 \mathrm{H}), 1.20(\mathrm{~s}, 9 \mathrm{H}), 3.72(\mathrm{~s}, 1 \mathrm{H}), 2.58-2.71(\mathrm{~m}, 1 \mathrm{H}), 4.67-4.88(\mathrm{~m}, 2 \mathrm{H}), 5.05(\mathrm{dd}, J=$ 15.8, 3.3 Hz, 1H), 5.15 - 5.25 (m, 2H), $5.67-5.84(\mathrm{~m}, 1 \mathrm{H}), 7.23-7.28$ (m, 1H), 7.33 $(\mathrm{dd}, J=8.2,1.2 \mathrm{~Hz}, 1 \mathrm{H}), 7.46(\mathrm{~d}, J=2.1 \mathrm{~Hz}, 1 \mathrm{H}) .{ }^{19} \mathrm{~F} \mathrm{NMR}\left(282 \mathrm{MHz}, \mathrm{CDCl}_{3}\right) \delta-84.97$ $(\mathrm{dd}, J=47.8,15.7 \mathrm{~Hz}, \mathrm{CF}) .{ }^{13} \mathrm{C} \mathrm{NMR}\left(75.5 \mathrm{MHz}, \mathrm{CDCl}_{3}\right) \delta 22.5(3 \mathrm{xCH}), 43.0\left(\mathrm{CH}_{2}\right)$, $53.1(\mathrm{CH}), 55.9(\mathrm{C}), 95.8\left(\mathrm{~d},{ }^{2} J_{C F}=22.2 \mathrm{~Hz}, \mathrm{CH}_{2}\right), 119.7\left(\mathrm{CH}_{2}\right), 127.6(\mathrm{CH}), 127.9(\mathrm{CH})$, $130.7\left(\mathrm{~d},{ }^{2} J_{C F}=27.2 \mathrm{~Hz}, \mathrm{C}\right), 131.1\left(\mathrm{~d},{ }^{3} J_{C F}=3.6 \mathrm{~Hz}, \mathrm{CH}\right), 133.8(\mathrm{CH}), 135.9(\mathrm{C}), 142.9$ 
(C), $162.3\left(\mathrm{~d},{ }^{1} J_{C F}=255.0 \mathrm{~Hz}, \mathrm{CF}\right)$. HRMS (EI) calcd for $\mathrm{C}_{16} \mathrm{H}_{21} \mathrm{NOFSCl}[\mathrm{M}+\mathrm{H}]^{+}$: 330.1089, found: 330.1094 .

\section{Experimental procedure for the protecting group exchange on compounds 18a,e}<smiles>[R]c1ccc(C(=C)F)c([C@H](CC=C)NS(=O)C(C)(C)C)c1</smiles>

18a: R: $\mathrm{H}$ 18e: $\mathrm{R}: \mathrm{Cl}$<smiles>[R]c1ccc(C(=C)F)c(C(CC=C)NC(C)=O)c1</smiles>

19a: R: $\mathrm{H},>99 \%$ 19e: $\mathrm{R}: \mathrm{Cl}, 78 \%$

To a solution of the corresponding starting material in $\mathrm{MeOH}(0.1 \mathrm{M}), \mathrm{HCl}(4 \mathrm{M}$ in dioxane, 10 equiv) was added at $0{ }^{\circ} \mathrm{C}$ and the reaction mixture was stirred for 1 hour. The solvents were removed under pressure and to the resulting residue anhydrous DCM $(0.055 \mathrm{M})$, pyridine $(0.37 \mathrm{M})$ and acetic anhydride $(0.55 \mathrm{M})$ were added. The reaction mixture was stirred at room temperature until completion as determined by TLC analysis (12 hours approx.). The solvents were then reduced under pressure and the residue purified by flash column chromatography, using a mixture of hexane:ethyl acetate as the eluent.

(Rotamers are observed in some of the ${ }^{1} \mathrm{H},{ }^{19} \mathrm{~F}$ as well as ${ }^{13} \mathrm{C}$ NMR spectra).

(S)- $N$-[1-(2-(1-Fluorovinil)fenil)but-3-en-1-il]acetamida (19a)<smiles>C=CC[C@H](NC(C)=O)c1ccccc1C(=C)F</smiles>

Following the experimental procedure, 19a was obtained as a colorless oil (25 $\mathrm{mg}$, $>99 \%$ ). $[\alpha]_{\mathrm{D}}^{25}=-42.8\left(\mathrm{c} 1.0 ; \mathrm{CHCl}_{3}\right) .{ }^{1} \mathrm{H}$ NMR $\left(300 \mathrm{MHz}, \mathrm{CDCl}_{3}\right) \delta 1.96(\mathrm{~s}, 3 \mathrm{H}), 2.47-$ $2.62(\mathrm{~m}, 2 \mathrm{H}), 4.80(\mathrm{dd}, J=48.8,3.1 \mathrm{~Hz}, 1 \mathrm{H}), 4.98-5.13(\mathrm{~m}, 3 \mathrm{H}), 5.28-5.39(\mathrm{~m}, 1 \mathrm{H})$, 5.70 (ddt, $J=17.1,10.1,7.0 \mathrm{~Hz}, 1 \mathrm{H}), 5.9(\mathrm{~s}, 1 \mathrm{H}), 7.22-7.41(\mathrm{~m}, 4 \mathrm{H}) .{ }^{19} \mathrm{~F}$ NMR $(282$ $\left.\mathrm{MHz}_{2} \mathrm{CDCl}_{3}\right) \delta-85.50(\mathrm{dd}, J=48.8,16.2 \mathrm{~Hz}, \mathrm{CF}) .{ }^{13} \mathrm{C} \mathrm{NMR}\left(75.5 \mathrm{MHz}, \mathrm{CDCl}_{3}\right) \delta 23.2$ $\left(\mathrm{CH}_{3}\right), 40.5\left(\mathrm{CH}_{2}\right), 50.8(\mathrm{CH}), 94.8\left(\mathrm{~d},{ }^{2} J_{C F}=22.5 \mathrm{~Hz}, \mathrm{CH}_{2}\right), 118.0\left(\mathrm{CH}_{2}\right), 126.8(\mathrm{CH})$, $127.2(\mathrm{CH}), 129.9(\mathrm{CH}), 130.2\left(\mathrm{~d},{ }^{3} J_{C F}=3.9 \mathrm{~Hz}, \mathrm{CH}\right), 131.4\left(\mathrm{~d},{ }^{2} J_{C F}=27.0 \mathrm{~Hz}, \mathrm{C}\right), 134.2$ 
(CH), $140.6(\mathrm{C}), 163.8\left(\mathrm{~d},{ }^{1} J_{C F}=254.3 \mathrm{~Hz}, \mathrm{CF}\right), 169.3(\mathrm{C})$. HRMS (EI) calcd for $\mathrm{C}_{14} \mathrm{H}_{16} \mathrm{NOF}[\mathrm{M}+\mathrm{H}]^{+}: 234.1289$, found: 234.1270 .

(S)- $N$-[1-(5-cloro-2-(1-Fluorovinil)fenil)but-3-en-1-il] acetamida (19e).<smiles>C=CCC(NC(C)=O)c1cc(Cl)ccc1C(=C)F</smiles>

Following the experimental procedure, 19e was obtained as a colorless oil (32 $\mathrm{mg}, 78 \%$ ). $[\alpha]_{\mathrm{D}}{ }^{25}=-40.3\left(\mathrm{c} 1.0 ; \mathrm{CHCl}_{3}\right) .{ }^{1} \mathrm{H}$ NMR $\left(300 \mathrm{MHz}, \mathrm{CDCl}_{3}\right) \delta 1.97$ (s, 3H), $2.36-2.62$ $(\mathrm{m}, 2 \mathrm{H}), 4.82(\mathrm{dd}, J=48.5,3.2 \mathrm{~Hz}, 1 \mathrm{H}), 5.03(\mathrm{dd}, J=16.2,3.2 \mathrm{~Hz}, 1 \mathrm{H}), 5.08-5.16(\mathrm{~m}$, 2H), $5.22-5.33(\mathrm{~m}, 1 \mathrm{H}), 5.69$ (ddt, $J=17.0,9.6,7.0 \mathrm{~Hz}, 1 \mathrm{H}), 6.03(\mathrm{~d}, J=6.4 \mathrm{~Hz}, 1 \mathrm{H})$, $7.19-7.24(\mathrm{~m}, 1 \mathrm{H}), 7.27-7.33(\mathrm{~m}, 2 \mathrm{H}) .{ }^{19} \mathrm{~F} \mathrm{NMR}\left(282 \mathrm{MHz}, \mathrm{CDCl}_{3}\right) \delta-86.34(\mathrm{dd}, J=$ 48.5, 16.1 Hz, CF). ${ }^{13} \mathrm{C}$ NMR $\left(75.5 \mathrm{MHz}, \mathrm{CDCl}_{3}\right) \delta 23.1\left(\mathrm{CH}_{3}\right), 40.4\left(\mathrm{CH}_{2}\right), 50.5(\mathrm{CH})$, $95.4\left(\mathrm{~d}, J=22.2 \mathrm{~Hz}, \mathrm{CH}_{2}\right), 118.6\left(\mathrm{CH}_{2}\right), 126.8(\mathrm{CH}), 127.3(\mathrm{CH}), 129.8\left(\mathrm{~d},{ }^{2} J_{C F}=27.7\right.$ $\mathrm{Hz}, \mathrm{C}), 131.4\left(\mathrm{~d},{ }^{3} J_{C F}=3.9 \mathrm{~Hz}, \mathrm{CH}\right), 133.7(\mathrm{CH}), 135.9(\mathrm{C}), 142.92(\mathrm{C}), 162.7$ (d, ${ }^{1} J_{C F}=$ $254.2 \mathrm{~Hz}, \mathrm{CF}), 169.6(\mathrm{C})$. HRMS (EI) calcd for $\mathrm{C}_{14} \mathrm{H}_{15} \mathrm{NOFCl}[\mathrm{M}+\mathrm{H}]^{+}: 268.0899$, found: 268.0899 .

\section{Experimental procedure for the synthesis of compounds 3a,e by RCM}<smiles>[R]c1ccc(C(=C)F)c([C@H](CC=C)NC(C)=O)c1</smiles>

19a: R: H

19e: $\mathrm{R}: \mathrm{Cl}$<smiles>[R]c1ccc2c(c1)[C@H](NC(C)=O)CC=C2F</smiles>

3a: R: H, $90 \%$

3e: $\mathrm{R}: \mathrm{Cl}, 86 \%$

Second generation Hoveyda-Grubbs catalyst (30 mol\%) was added to a solution of the corresponding starting material in anhydrous toluene $(0.1 \mathrm{M})$ and the reaction mixture was stirred at $100{ }^{\circ} \mathrm{C}$ for 4 hours. The residue obtained after the solvent was removed under reduced pressure was purified by flash chromatography on silica gel using mixtures of hexane and ethyl acetate as eluents. The enantiomeric excess of the product was determined by HPLC: Chiralcel OD-H $(25 \mathrm{~cm} \quad x \quad 0.46 \mathrm{~cm}$ column $)$, hexane:isopropanol 95:5 as eluent and flow $=1 \mathrm{ml} / \mathrm{min}$. 
<smiles>CC(=O)NC1CC=C(F)c2ccccc21</smiles>

Following the experimental procedure, 3a was obtained as a colorless oil (15 mg, 90\%). The spectroscopic data for 3a may be found in pages 8-9. The enantiomeric excess was determined as described in the procedure: $t_{\mathrm{R} \text { minor }}=20.6 \mathrm{~min}, t_{\mathrm{R} \text { major }}=23.4 \mathrm{~min}$.

(S)-N-(7-Cloro-4-fluoro-1,2-dihidronaftalen-1-il)acetamida (3e)<smiles>CC(=O)NC1CC=C(F)c2ccc(Cl)cc21</smiles>

Following the experimental procedure, $3 \mathbf{e}$ was obtained as a colorless oil (18 $\mathrm{mg}, 86 \%)$. $[\alpha]_{\mathrm{D}}^{25}=-138.6\left(\mathrm{c} 1.0 ; \mathrm{CHCl}_{3}\right) .{ }^{1} \mathrm{H}$ NMR $\left(300 \mathrm{MHz}, \mathrm{CDCl}_{3}\right) \delta 1.98(\mathrm{~s}, 3 \mathrm{H}), 2.41-2.54$ (m, 1H), $2.60-2.73(\mathrm{~m}, 1 \mathrm{H}), 5.20(\mathrm{ddd}, J=8.5,6.4 \mathrm{~Hz}, 6.4 \mathrm{~Hz}, 1 \mathrm{H}), 5.44(\mathrm{ddd}, J=13.1$, 5.1, 4.2 Hz, 1H), 5.98 (d, J=7.5 Hz, 1H), $7.27-7.37$ (m, 3H). ${ }^{19} \mathrm{~F}$ NMR (282 MHz, $\left.\mathrm{CDCl}_{3}\right) \delta-125.33--125.49(\mathrm{CF}) .{ }^{13} \mathrm{C} \mathrm{NMR}\left(75.5 \mathrm{MHz}, \mathrm{CDCl}_{3}\right) \delta 23.38\left(\mathrm{CH}_{3}\right), 28.2(\mathrm{~d}$, $\left.{ }^{3} J_{C F}=7.0 \mathrm{~Hz}, \mathrm{CH}_{2}\right), 45.7(\mathrm{CH}), 100.6\left(\mathrm{~d},{ }^{2} J_{C F}=16.4 \mathrm{~Hz}, \mathrm{CH}\right), 122.6\left(\mathrm{~d},{ }^{3} J_{C F}=4.0 \mathrm{~Hz}\right.$, $\mathrm{CH}), 126.6\left(\mathrm{~d},{ }^{2} J_{C F}=26.7 \mathrm{~Hz}, \mathrm{C}\right), 127.4\left(\mathrm{~d},{ }^{4} J_{C F}=3.5 \mathrm{~Hz}, \mathrm{CH}\right), 128.3(\mathrm{CH}), 134.8(\mathrm{C})$, $137.5\left(\mathrm{~d},{ }^{3} J_{C F}=6.4 \mathrm{~Hz}, \mathrm{C}\right), 155.5\left(\mathrm{~d},{ }^{1} J_{C F}=251.7 \mathrm{~Hz}, \mathrm{CF}\right), 169.3(\mathrm{C})$. HRMS (EI) calcd for $\mathrm{C}_{12} \mathrm{H}_{11} \mathrm{NOFCl} \quad[\mathrm{M}+\mathrm{H}]^{+}: 240.0586$, found: 240.0581. The enantiomeric excess was determined as described in the procedure: $t_{\mathrm{R} \text { minor }}=16.0 \mathrm{~min}, t_{\mathrm{R} \text { major }}=18.8 \mathrm{~min}$.

\section{Experimental procedure for the deprotection of compounds 12a,e}<smiles>[X]c1ccc2c(c1)C(NC(=O)C(F)(F)F)CC=C2F</smiles><smiles>[X]c1ccc2c(c1)[C@@H](N)CC=C2F</smiles>

12a, $\mathrm{X}=\mathrm{H}(99 \%$ ee $)$

20a, $\mathrm{X}=\mathrm{H}(70 \%, 98 \%$ ee $)$

$12 \mathrm{e}, \mathrm{X}=\mathrm{Cl}(>99 \%$ ee $)$

20e, $X=\mathrm{Cl}(80 \%,>99 \%$ ee $)$ 
An aqueous solution of $\mathrm{K}_{2} \mathrm{CO}_{3}$ in water was added to a solution of the corresponding trifluoroacetamide $12 \mathrm{in} \mathrm{MeOH}$ at room temperature. The reaction mixture was stirred at $60{ }^{\circ} \mathrm{C}$ until disappearance of the starting material, as judged by TLC analysis ( $3 \mathrm{~h}$ approx.). Then, the reaction was quenched with water, extracted with AcOEt (3 times), the combined organic layers were dried over anhydrous $\mathrm{Na}_{2} \mathrm{SO}_{4}$, flitered and concentrated under vacuum. The residue was purified by flash chromatography on silica gel using DCM:MeOH (5:1) as eluent. The enantiomeric excess of the product was determined by HPLC.

(S)-4-Fluoro-1,2-dihydronaphthalen-1-amine (20a)<smiles>NC1CC=C(F)c2ccccc21</smiles>

Following the experimental procedure, 20a was obtained as a colorless oil (19 $\mathrm{mg}, 70 \%$ ). $[\alpha]_{D^{25}}^{25}-70.8\left(\mathrm{c} 1.0 ; \mathrm{CHCl}_{3}\right) .{ }^{1} \mathrm{H}$ NMR $\left(300 \mathrm{MHz}, \mathrm{CDCl}_{3}\right) \delta 2.39-2.50(\mathrm{~m}, 1 \mathrm{H}), 2.61-$ $2.73(\mathrm{~m}, 1 \mathrm{H}), 4.03(\mathrm{t}, J=6.3 \mathrm{~Hz}, 1 \mathrm{H}), 5.42(\mathrm{ddd}, J=13.5,5.1,4.2 \mathrm{~Hz}, 1 \mathrm{H}), 7.29-7.33$ (m, 2H), $7.36-7.47(\mathrm{~m}, 2 \mathrm{H}) .{ }^{19} \mathrm{~F}$ NMR $\left(282 \mathrm{MHz}, \mathrm{CDCl}_{3}\right) \delta-127.05$ (dtd, J = 11.3, 5.6, $2.8 \mathrm{~Hz}) .{ }^{13} \mathrm{C}$ NMR $\left(75.5 \mathrm{MHz}, \mathrm{CDCl}_{3}\right) \delta 30.8\left(\mathrm{~d},{ }^{3} J_{\mathrm{CF}}=5.6 \mathrm{~Hz}, \mathrm{CH}_{2}\right), 48.8(\mathrm{CH}), 100.1$ $\left(\mathrm{d},{ }^{2} J_{C F}=16.1 \mathrm{~Hz}, \mathrm{CH}\right), 121.1\left(\mathrm{~d},{ }^{4} J_{C F}=4.3, \mathrm{CH}\right), 126.1\left(\mathrm{~d},{ }^{3} J_{C F}=5.0 \mathrm{~Hz}, \mathrm{C}\right), 127.5$ $(\mathrm{CH}), 129.0(\mathrm{CH}), 155.8\left(\mathrm{~d},{ }^{1} J_{C F}=249.5 \mathrm{~Hz}, \mathrm{CF}\right)$. HRMS (EI) calcd for $\mathrm{C}_{12} \mathrm{H}_{11} \mathrm{NOFCl}$ $[\mathrm{M}+\mathrm{H}]^{+}:$164.0870, found: 164.0863 . The enantiomeric excess was determined by HPLC: Chiralpak IC (25 cm x $0.46 \mathrm{~cm}$ column), hexane:isopropanol 98:2 as eluent and flow $=1$ $\mathrm{ml} / \mathrm{min} .: t_{\mathrm{R}}$ major $=25.4 \mathrm{~min}, t_{\mathrm{R}} \operatorname{minor}=28.3 \mathrm{~min}$.

\section{(S)-7-Chloro-4-fluoro-1,2-dihydronaphthalen-1-amine (20e)}<smiles>N[C@H]1CC=C(F)c2ccc(Cl)cc21</smiles>

Following the experimental procedure, 20a was obtained as a colorless oil ( $9 \mathrm{mg}, 80 \%)$. $[\alpha]_{\mathrm{D}}{ }^{25}=-28.6\left(\mathrm{c} 1.0 ; \mathrm{CHCl}_{3}\right) .{ }^{1} \mathrm{H} \mathrm{NMR}\left(300 \mathrm{MHz}, \mathrm{CDCl}_{3}\right) \delta 2.35-2.46(\mathrm{~m}, 1 \mathrm{H}), 2.60-$ $2.70(\mathrm{~m}, 1 \mathrm{H}), 3.98$ (t, $J=7.0 \mathrm{~Hz}, 1 \mathrm{H}), 5.44$ (dt, $J=13.5,4.8 \mathrm{~Hz}, 1 \mathrm{H}), 7.24-7.28$ (m, 1H), $7.33-7.39(\mathrm{~m}, 2 \mathrm{H}) .{ }^{19} \mathrm{~F}$ NMR $\left(282 \mathrm{MHz}, \mathrm{CDCl}_{3}\right) \delta-126.8(\mathrm{dtd}, \mathrm{J}=14.1,5.6,2.8$ $\mathrm{Hz}) .{ }^{13} \mathrm{C}$ NMR $\left(75.5 \mathrm{MHz}, \mathrm{CDCl}_{3}\right) \delta 31.3\left(\mathrm{~d},{ }^{3} \mathrm{~J}_{\mathrm{CF}}=6.8 \mathrm{~Hz}, \mathrm{CH}_{2}\right), 48.8(\mathrm{CH}), 100.9(\mathrm{~d}$, 
$\left.{ }^{2} J_{C F}=15.8 \mathrm{~Hz}, \mathrm{CH}\right), 122.5(\mathrm{CH}), 126.2\left(\mathrm{~d},{ }^{3} J_{C F}=4.2 \mathrm{~Hz}, \mathrm{C}\right), 127.4(\mathrm{CH}), 134.8(\mathrm{C})$, $142.3\left(\mathrm{~d},{ }^{3} J_{C F}=6.4 \mathrm{~Hz}, \mathrm{C}\right), 155.3\left(\mathrm{~d},{ }^{1} J_{C F}=249.4 \mathrm{~Hz}, \mathrm{CF}\right)$. HRMS (EI) calcd for $\mathrm{C}_{12} \mathrm{H}_{11} \mathrm{NOFCl}[\mathrm{M}+\mathrm{H}]^{+}:$198.0480, found: 198.0478. The enantiomeric excess was determined by HPLC: Chiralpak OD-H (25 cm x $0.46 \mathrm{~cm}$ column), hexane:isopropanol 99.8:0.2 as eluent and flow $=1.5 \mathrm{ml} / \mathrm{min} .: t_{\mathrm{R} \text { major }}=18.7 \mathrm{~min}, t_{\mathrm{R} \text { minor }}=20.5 \mathrm{~min}$.

NOESY 2D-NMR of $N$-[(1S,3S,4S)-3-bromo-4-fluoro-1,2,3,4-tetrahidronaphthalen1-yl]acetamide (2a).
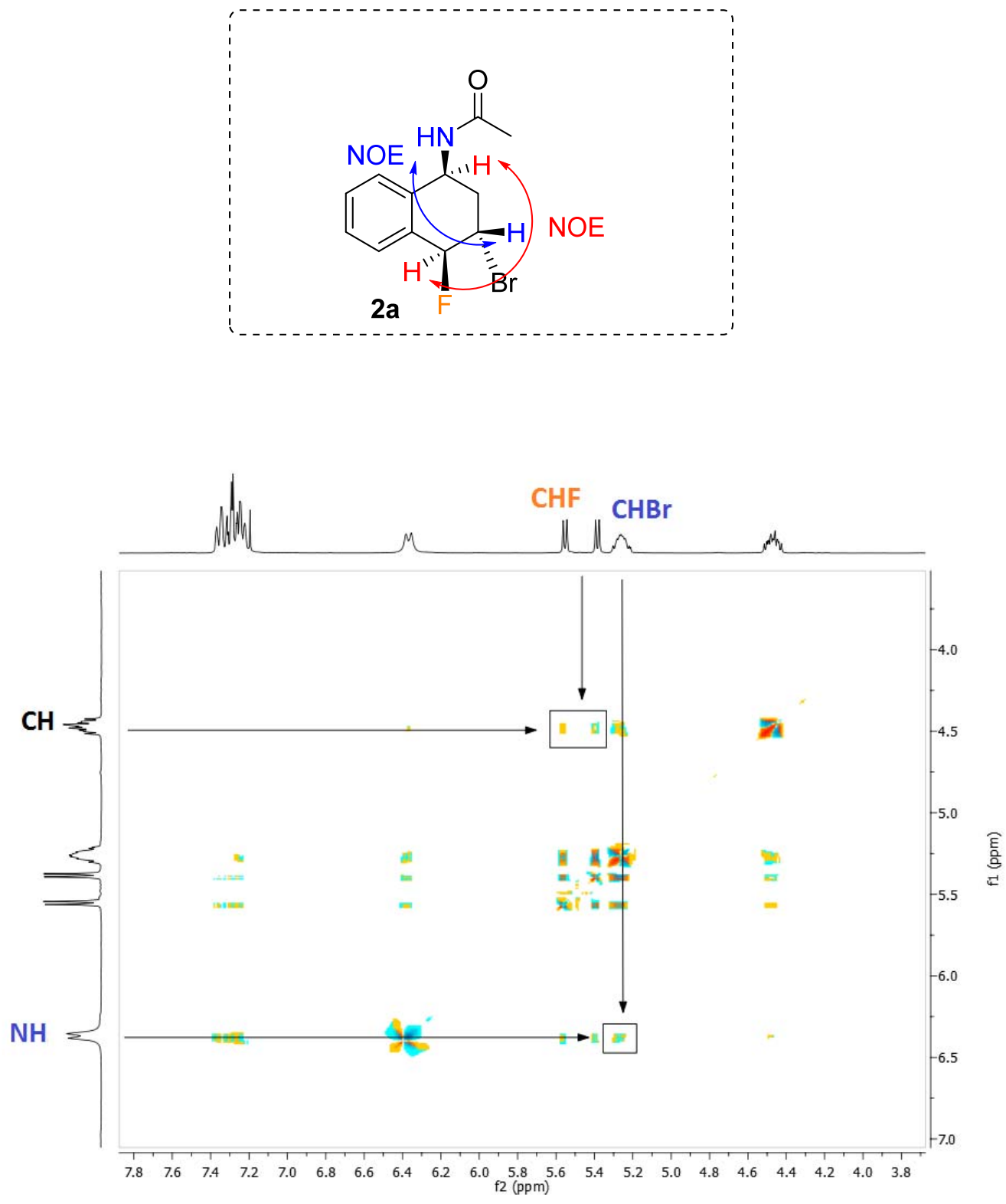


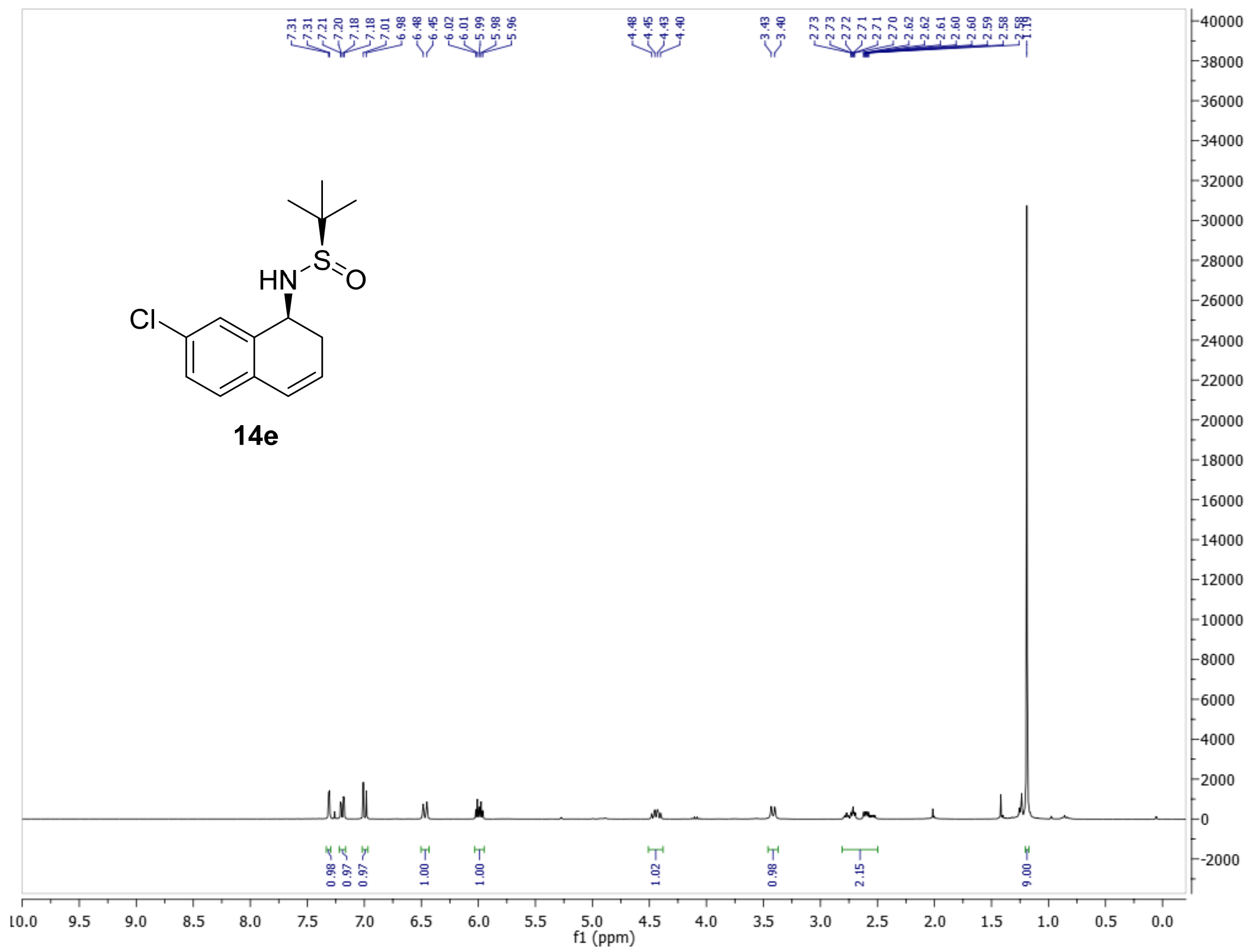




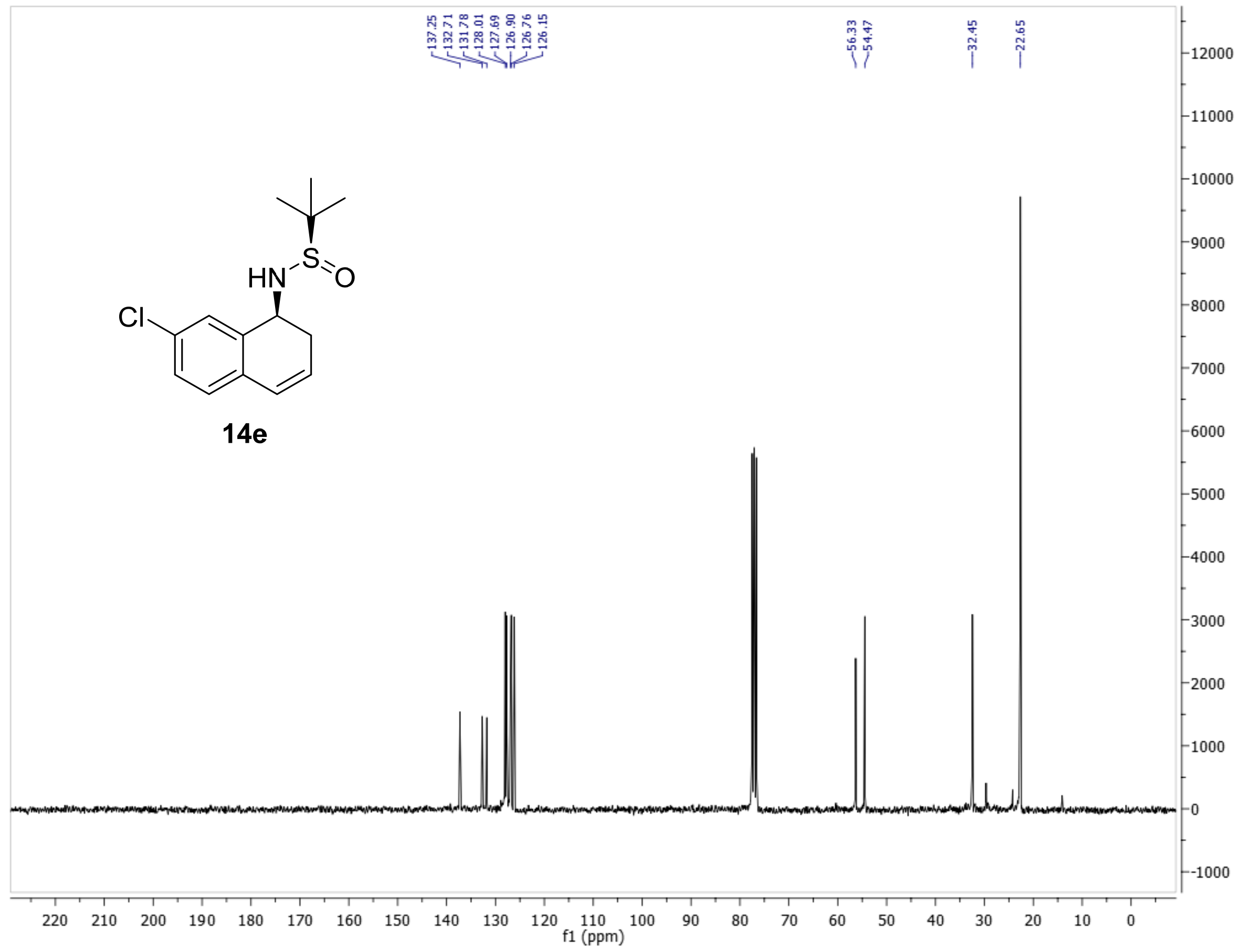




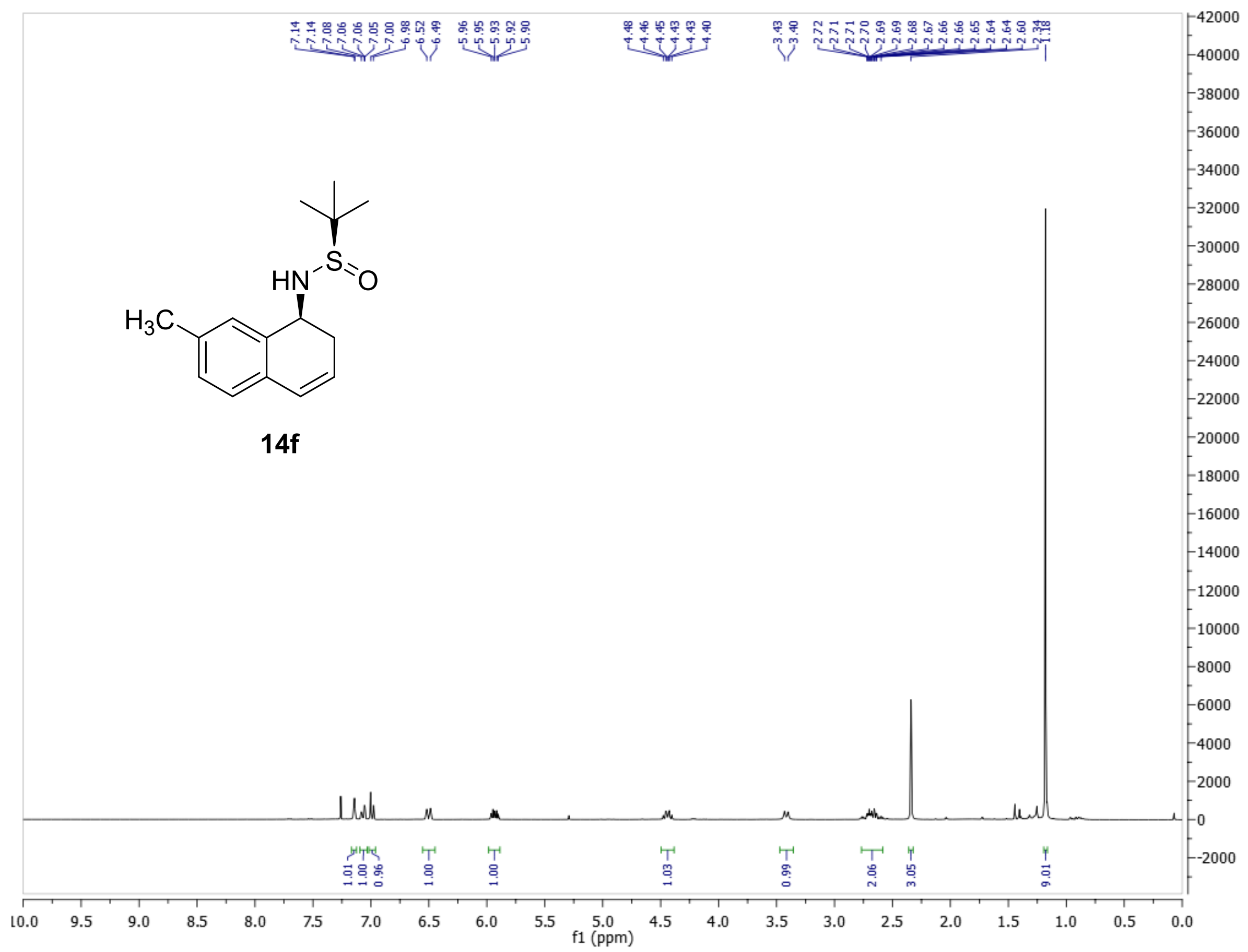




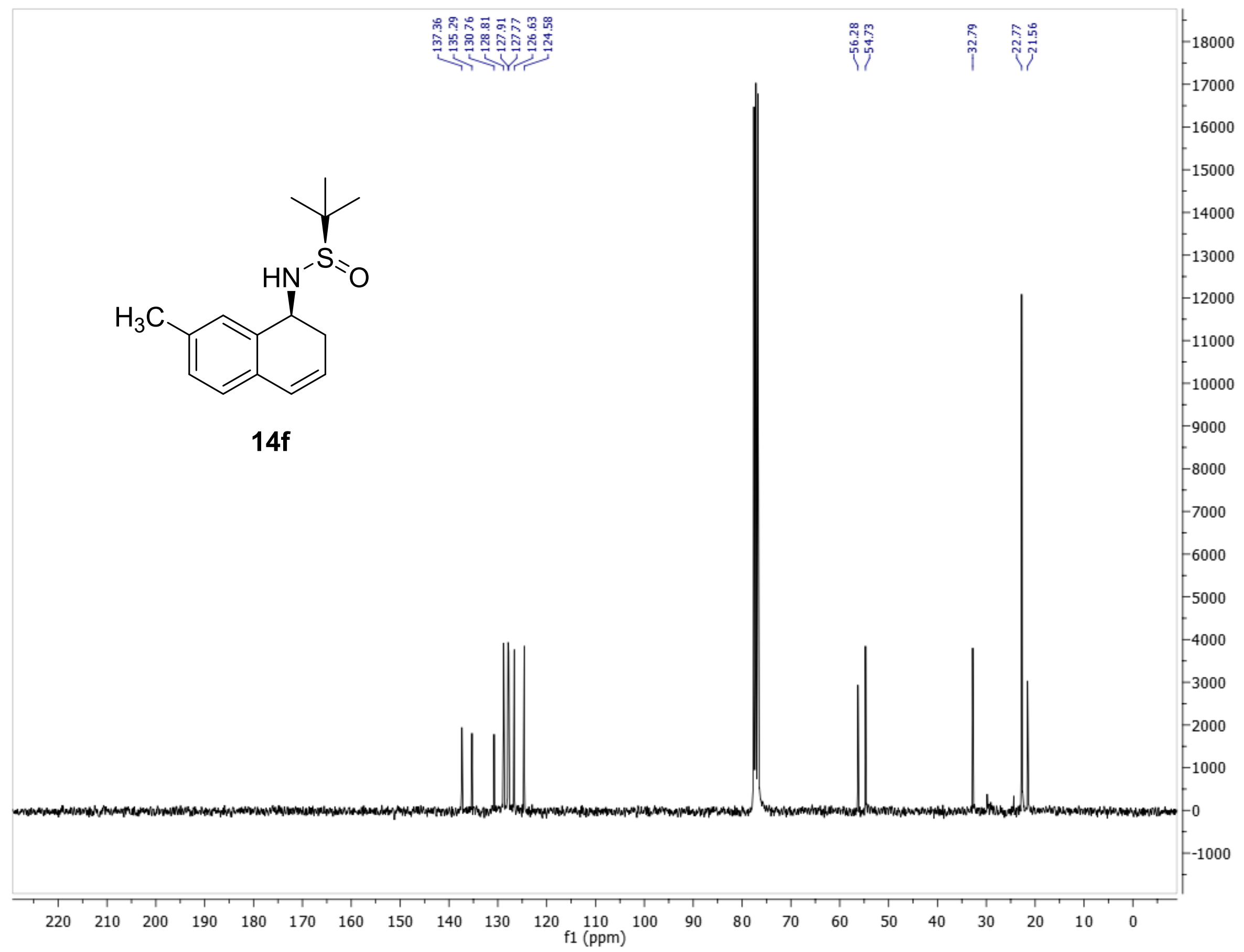




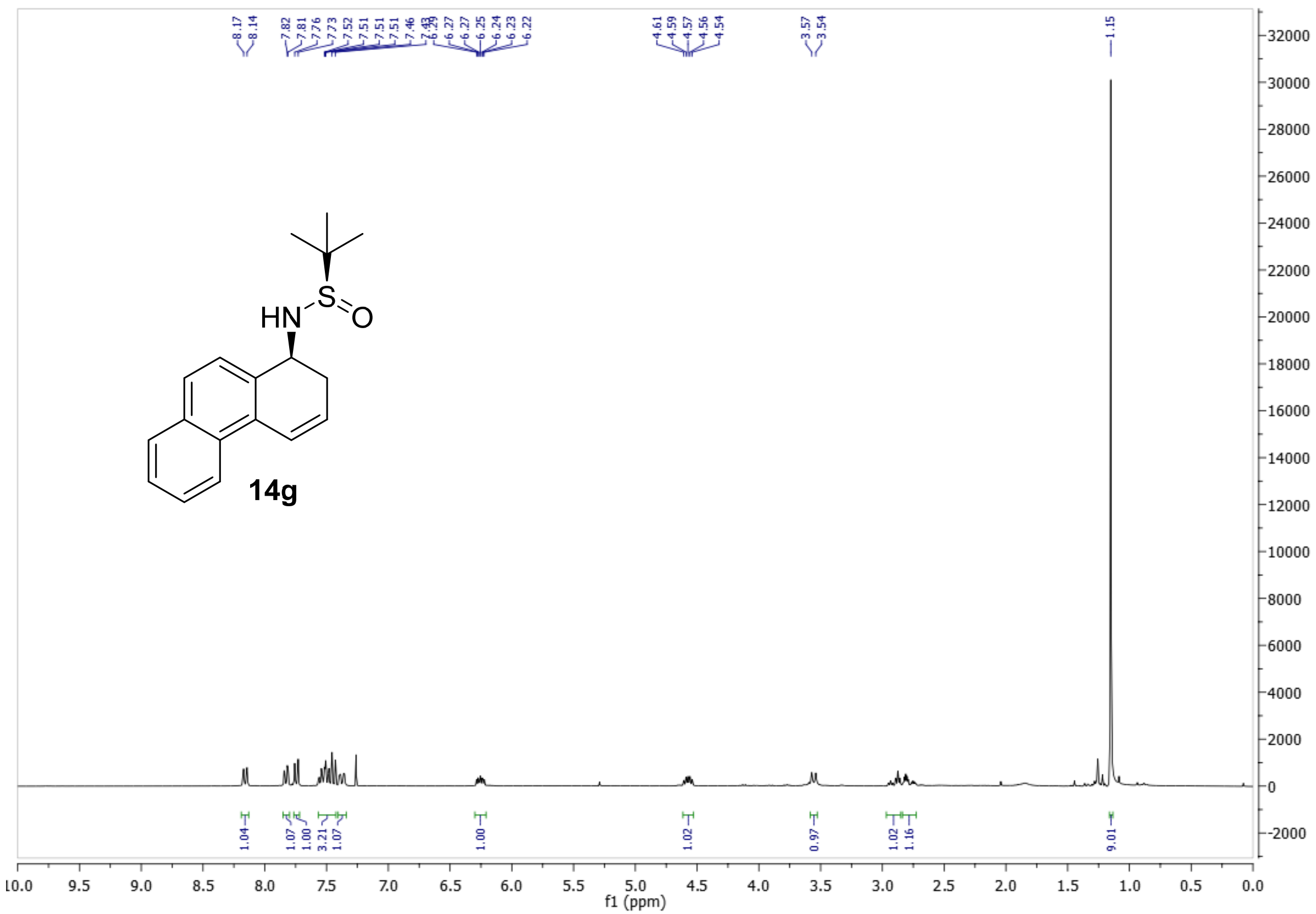




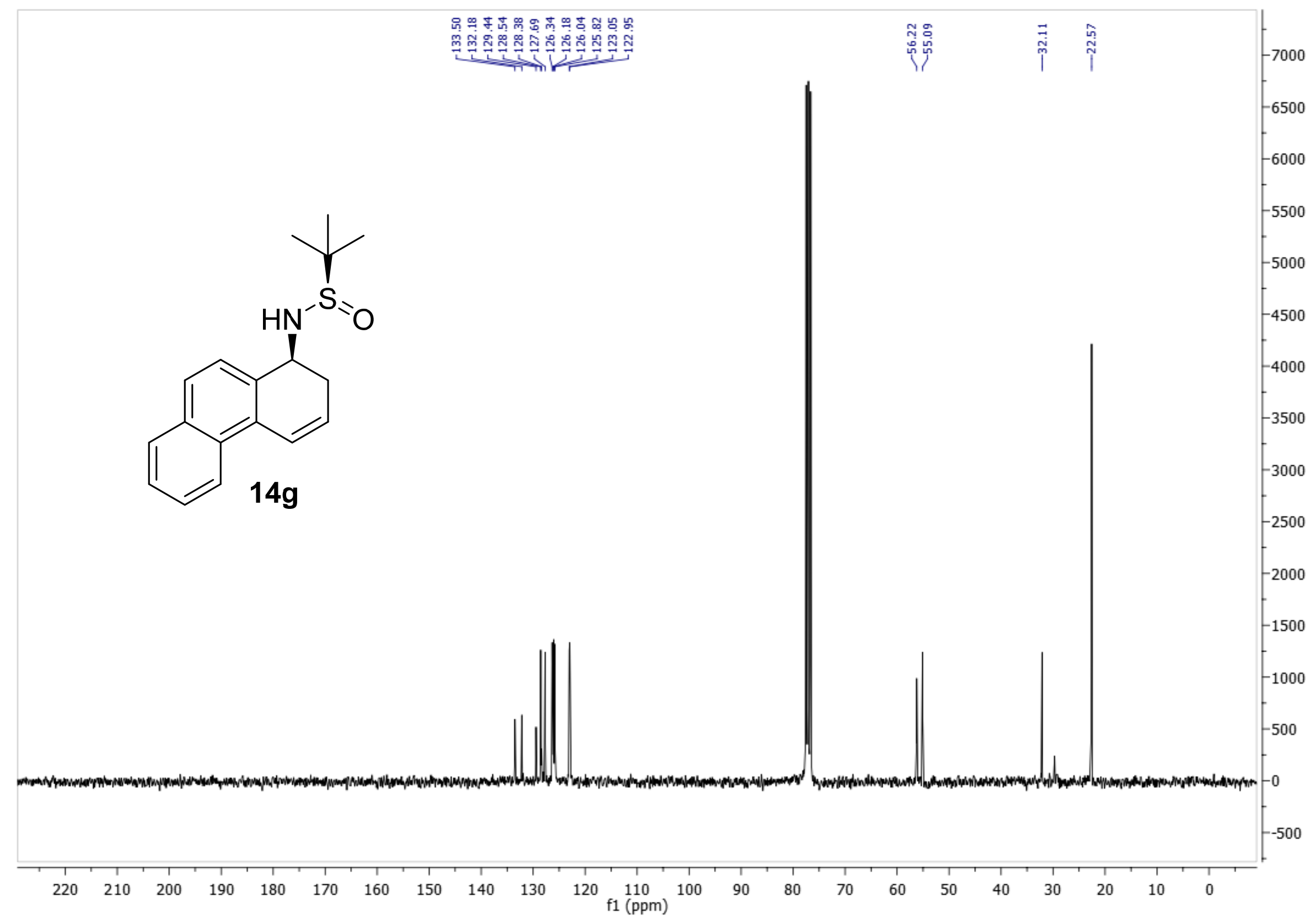




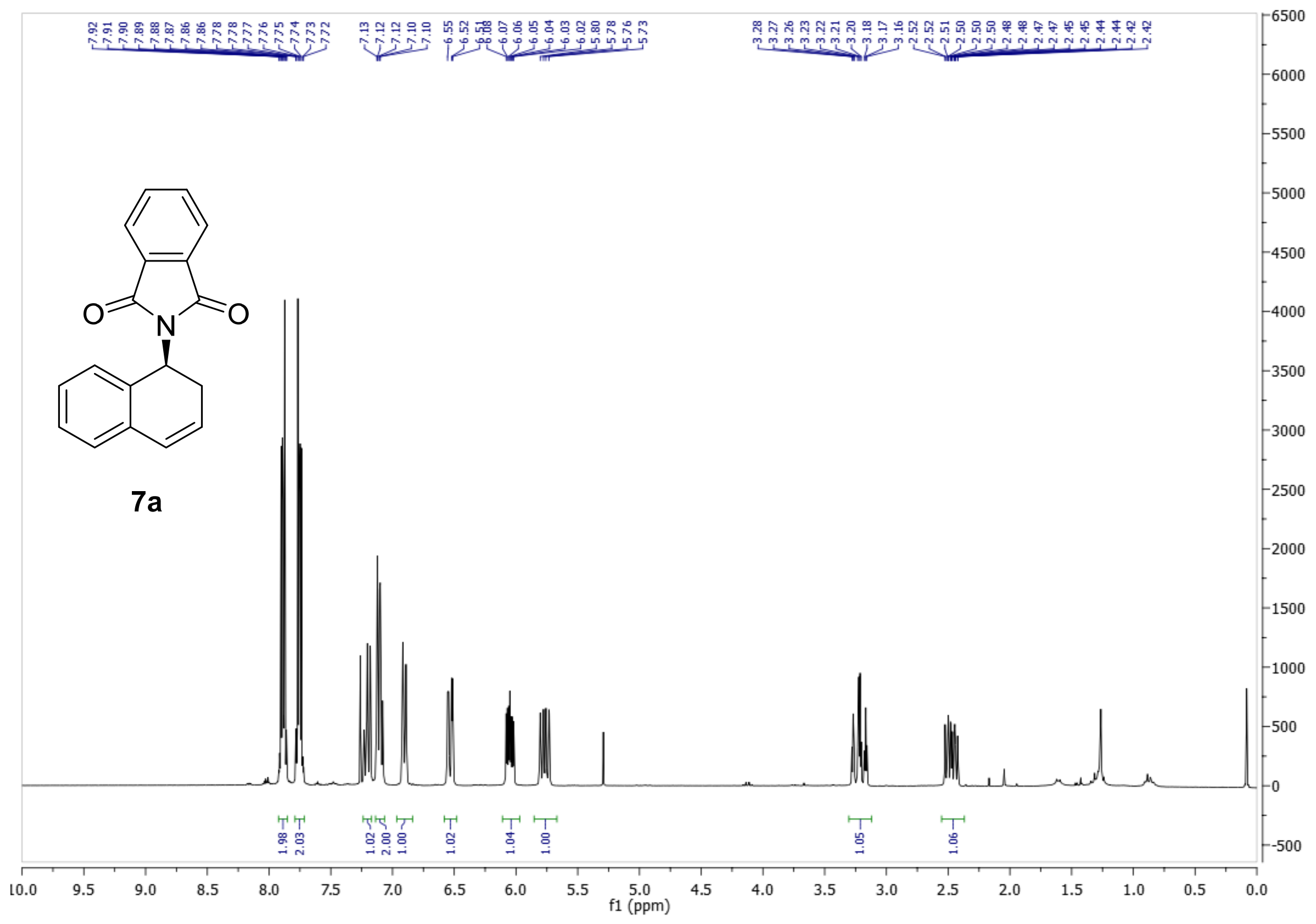




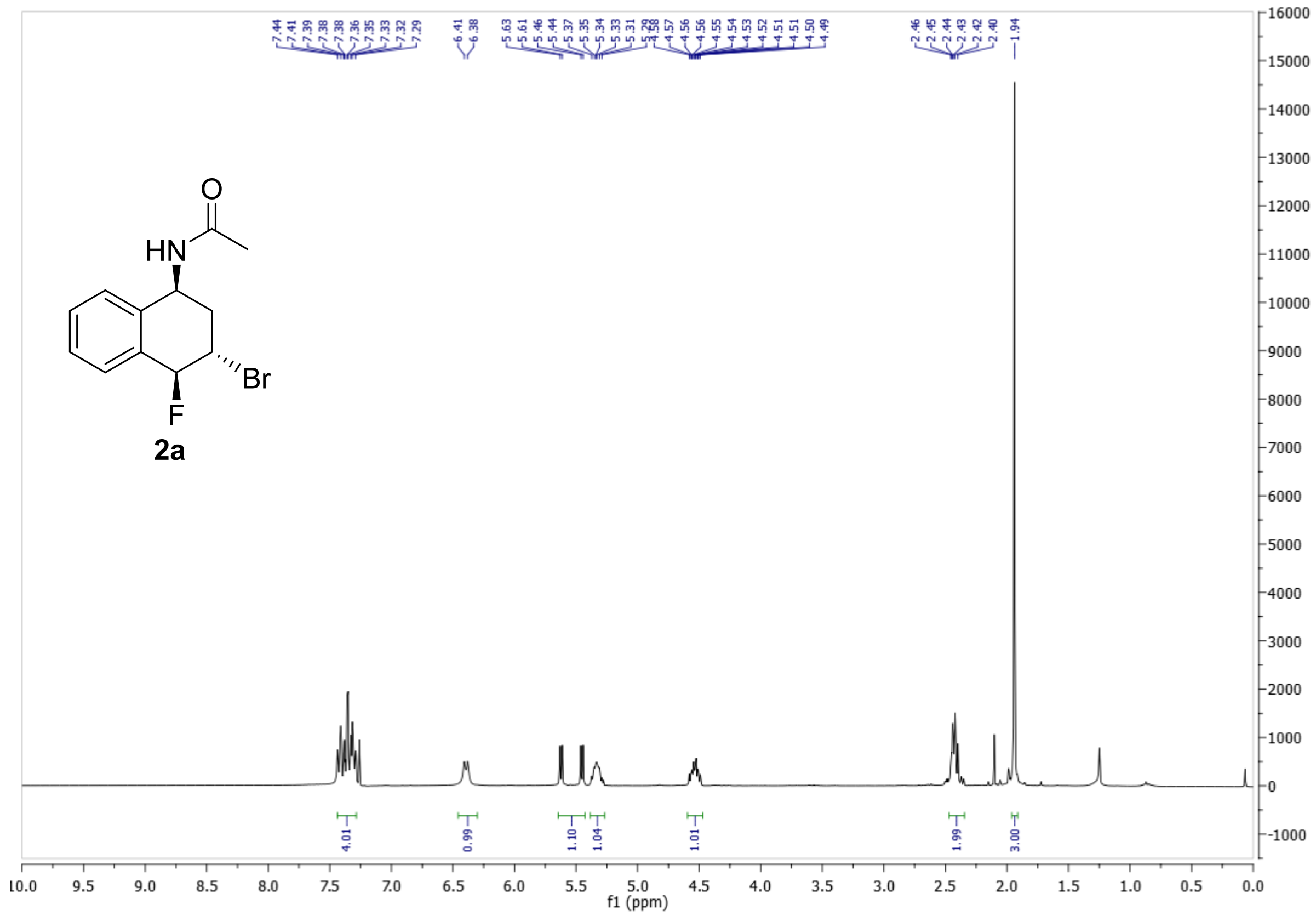




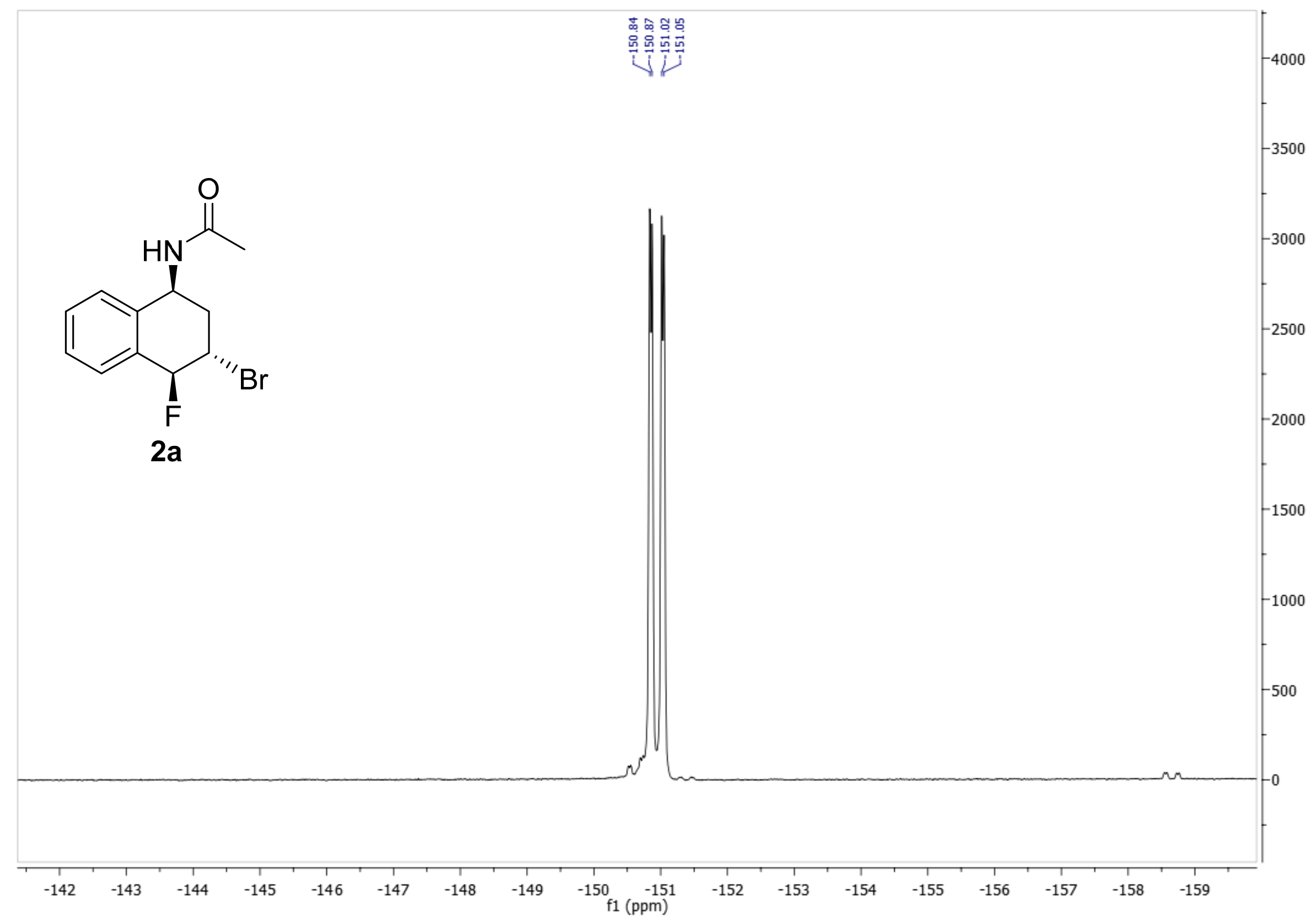




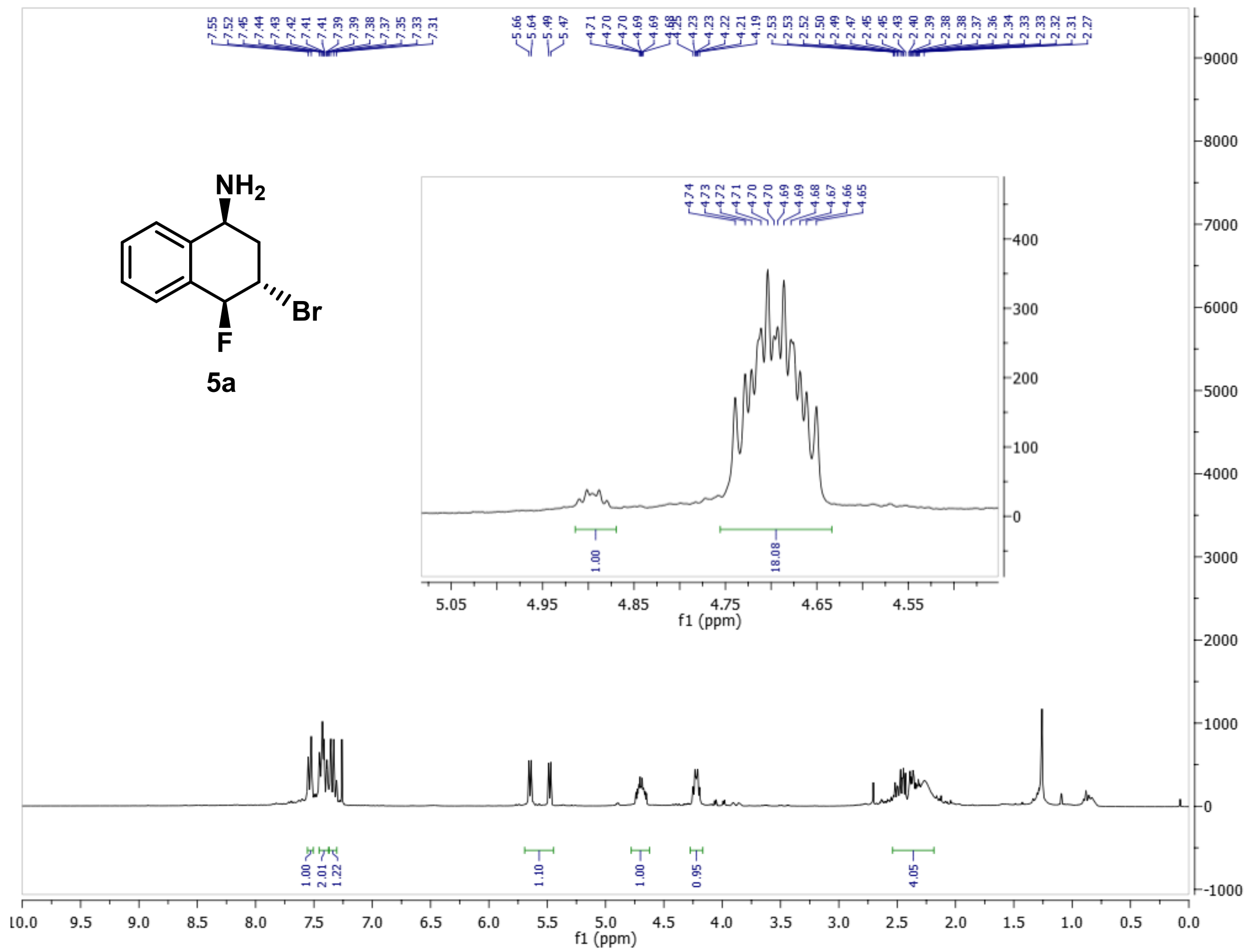




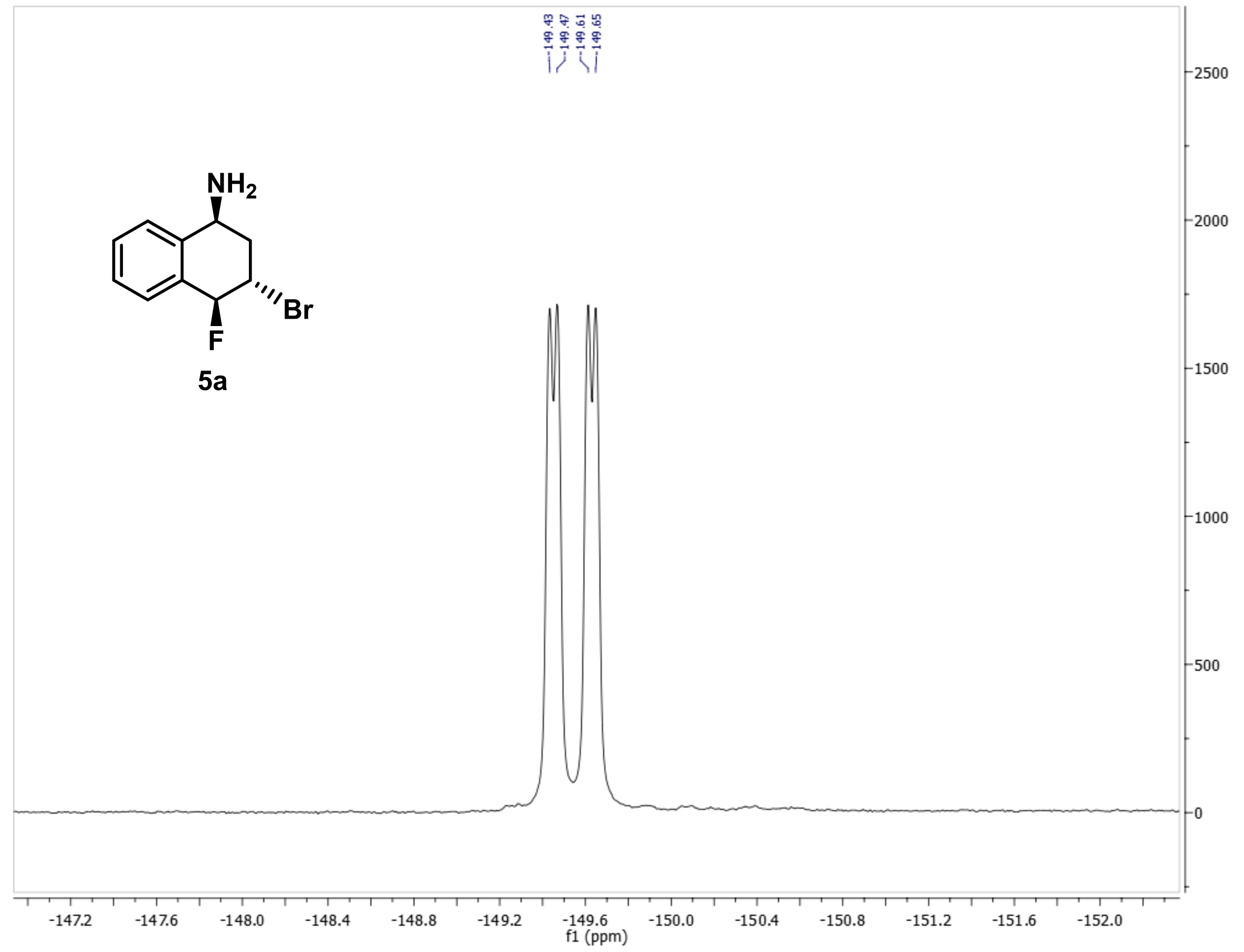




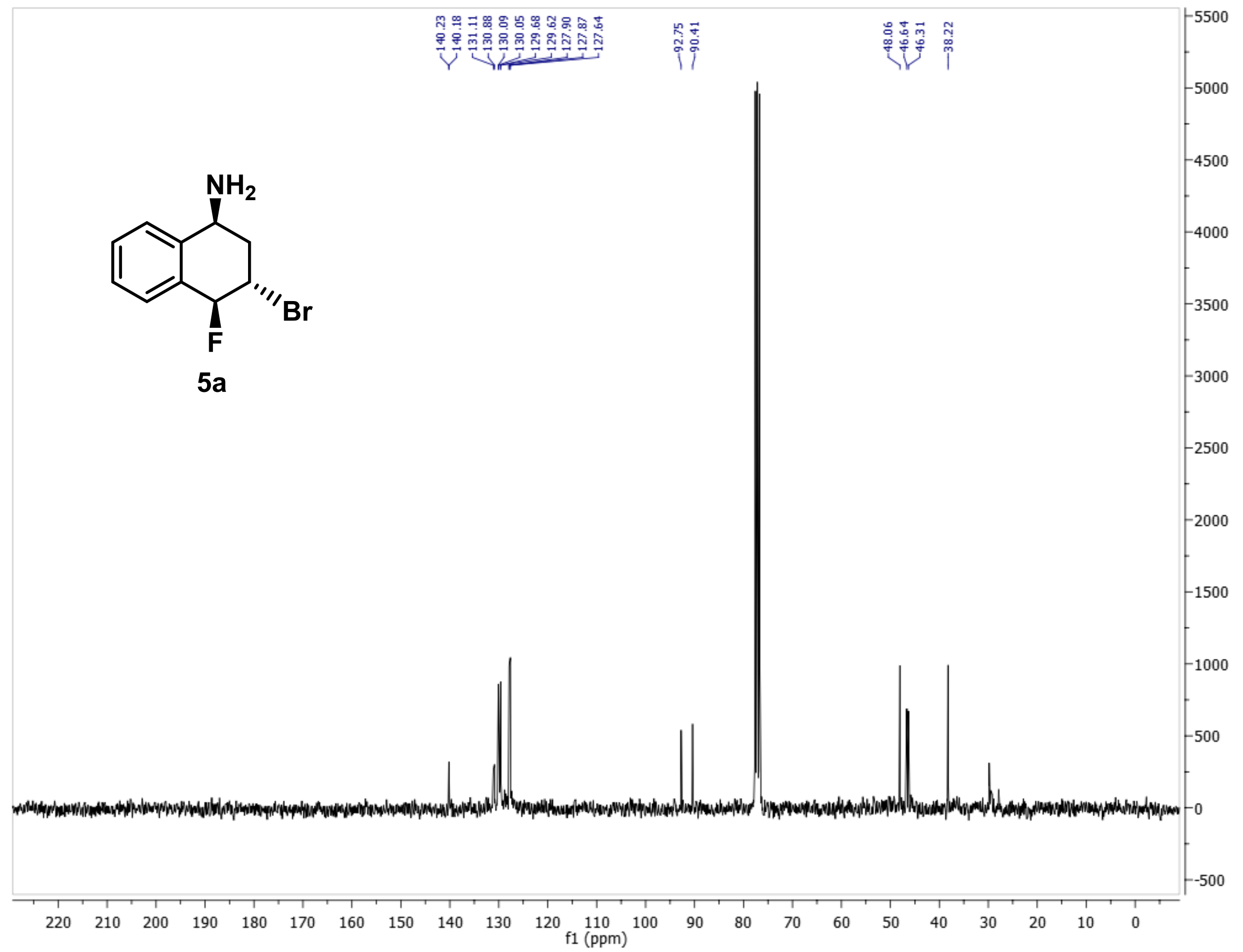




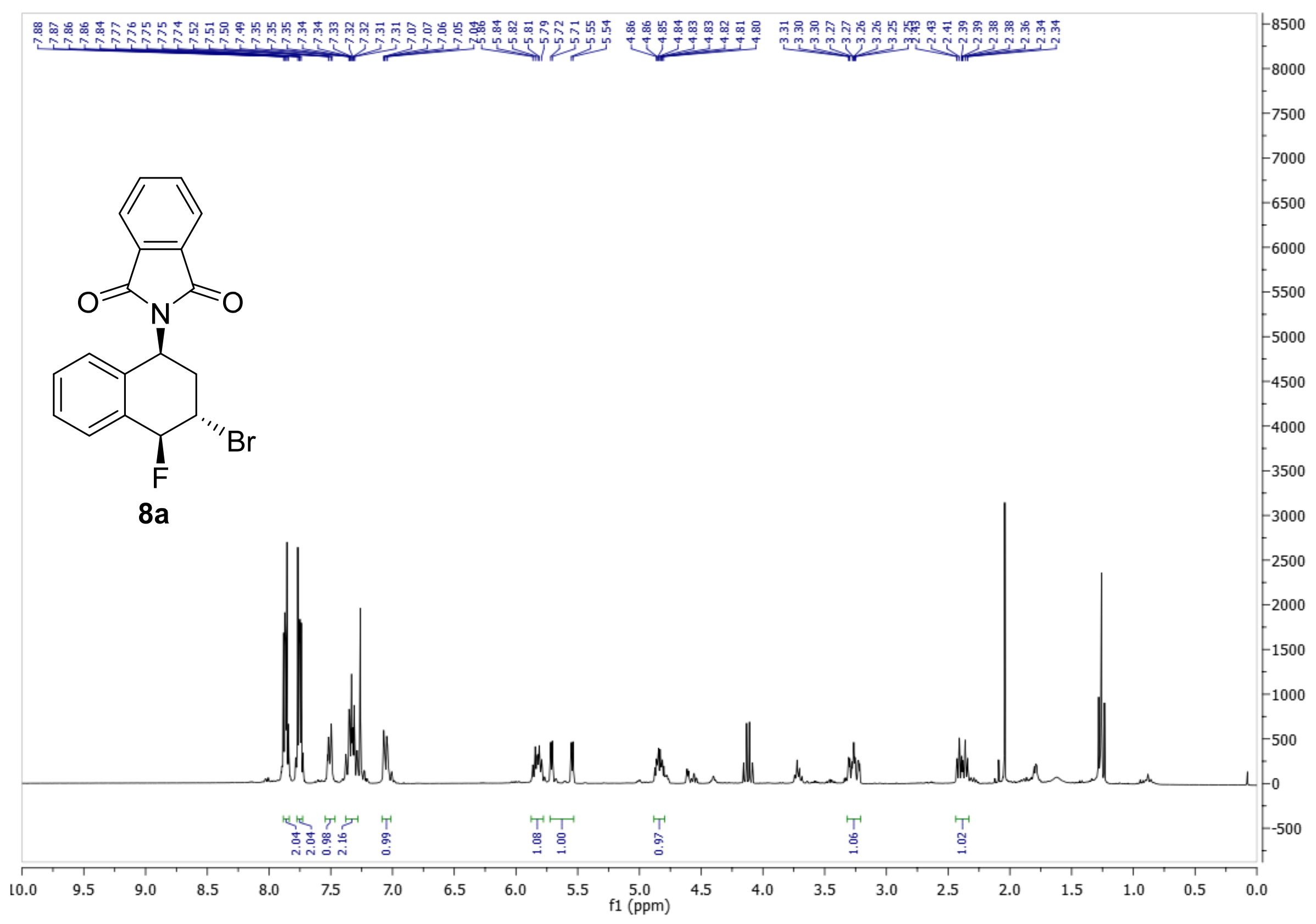




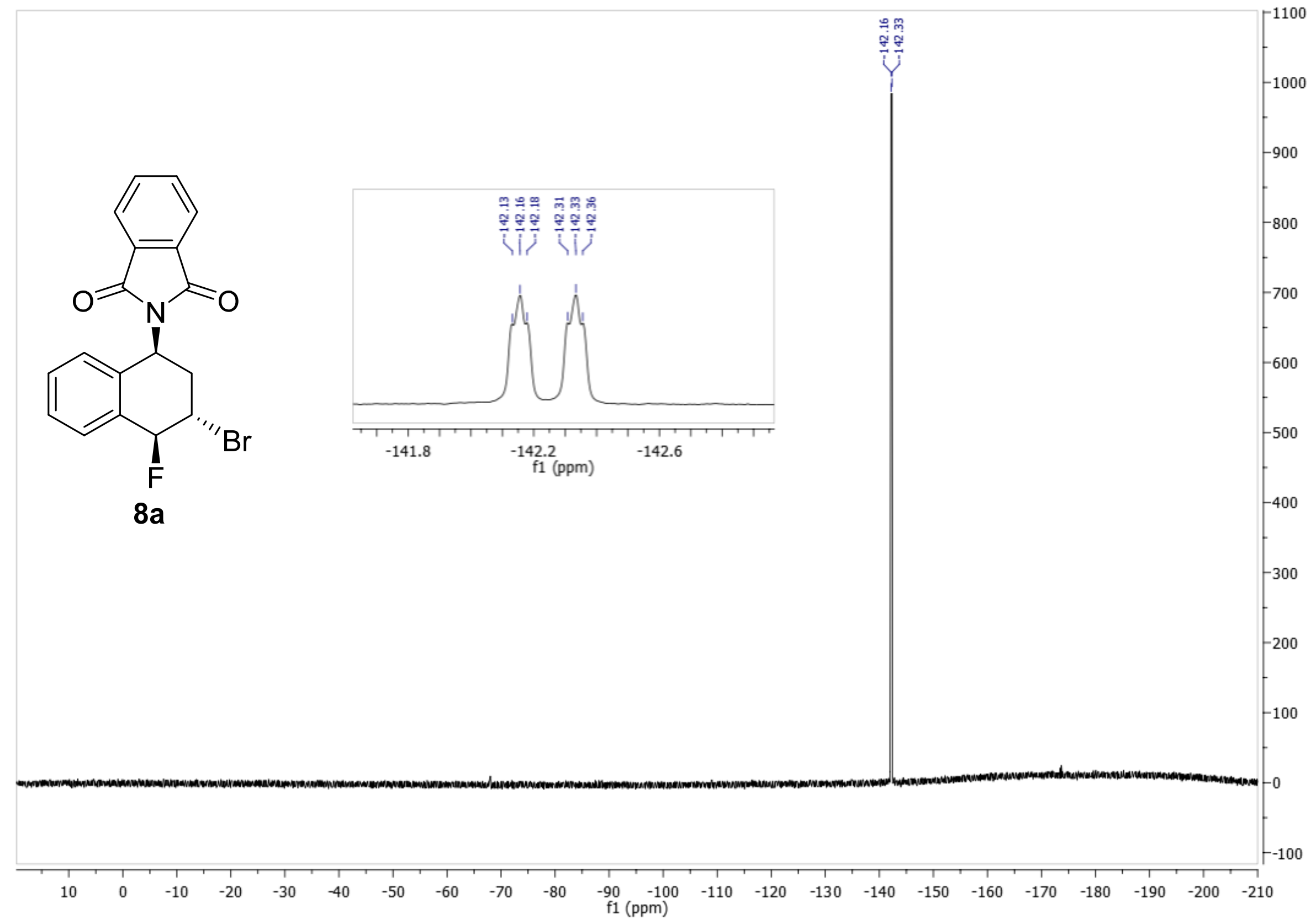




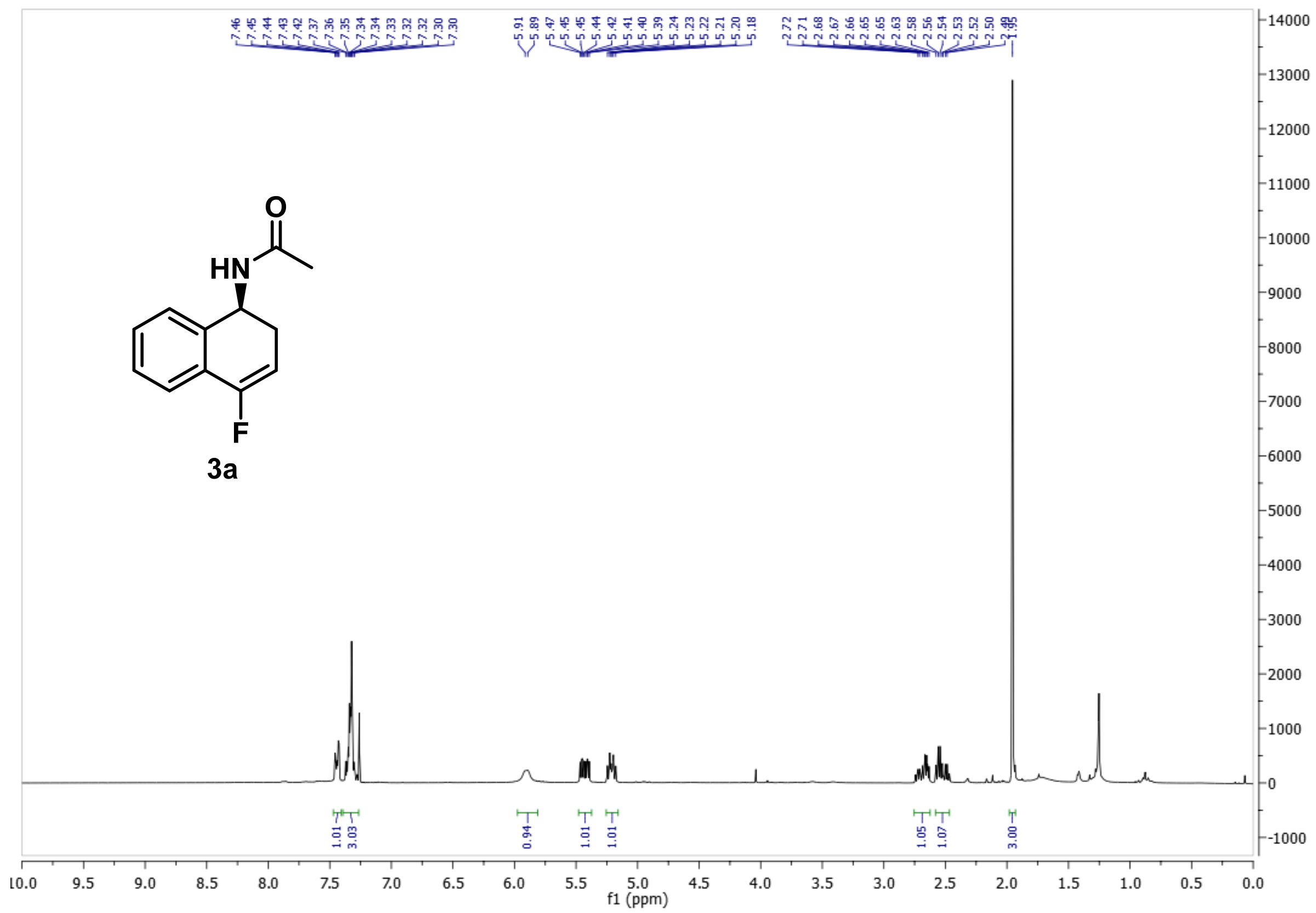




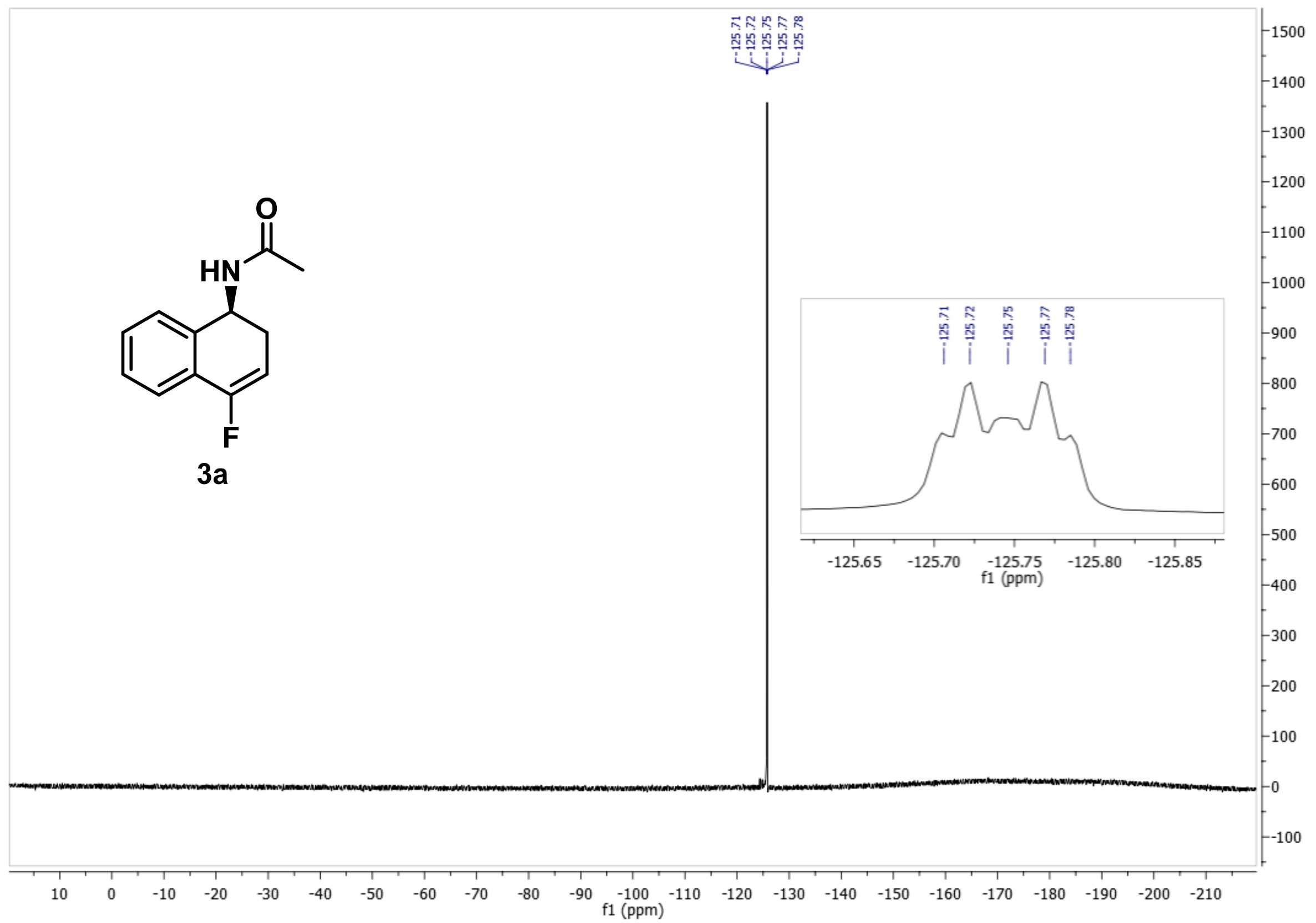




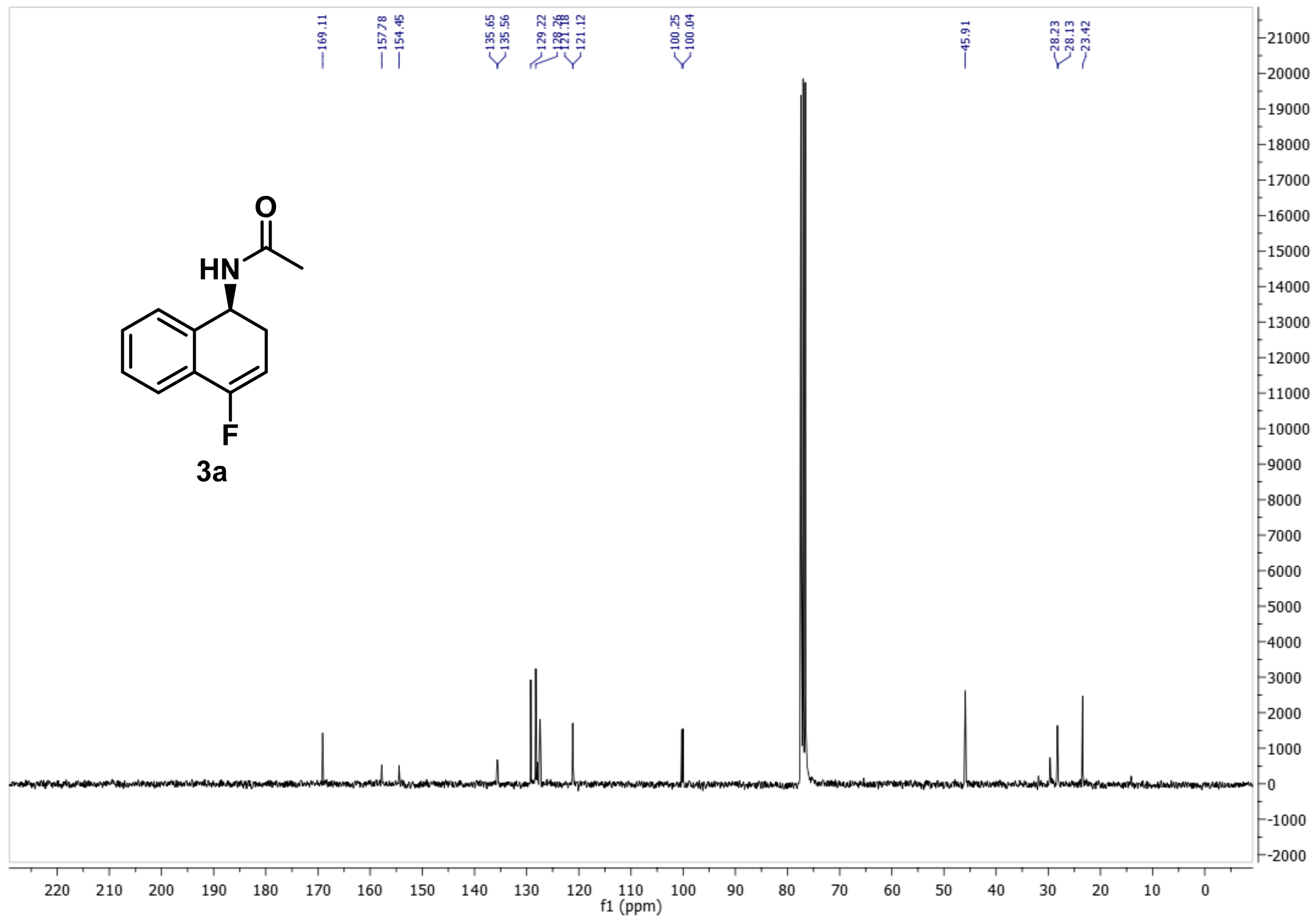




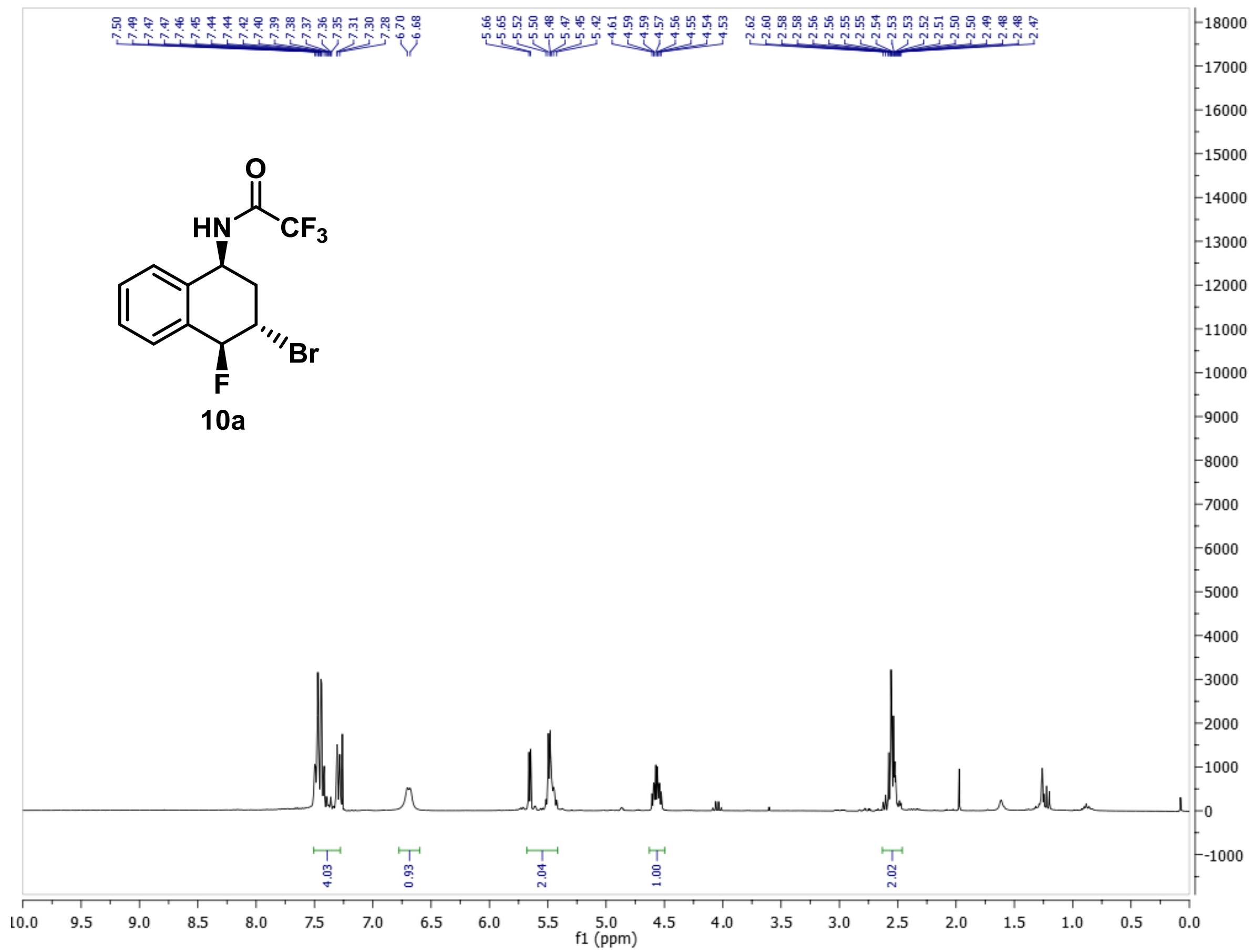




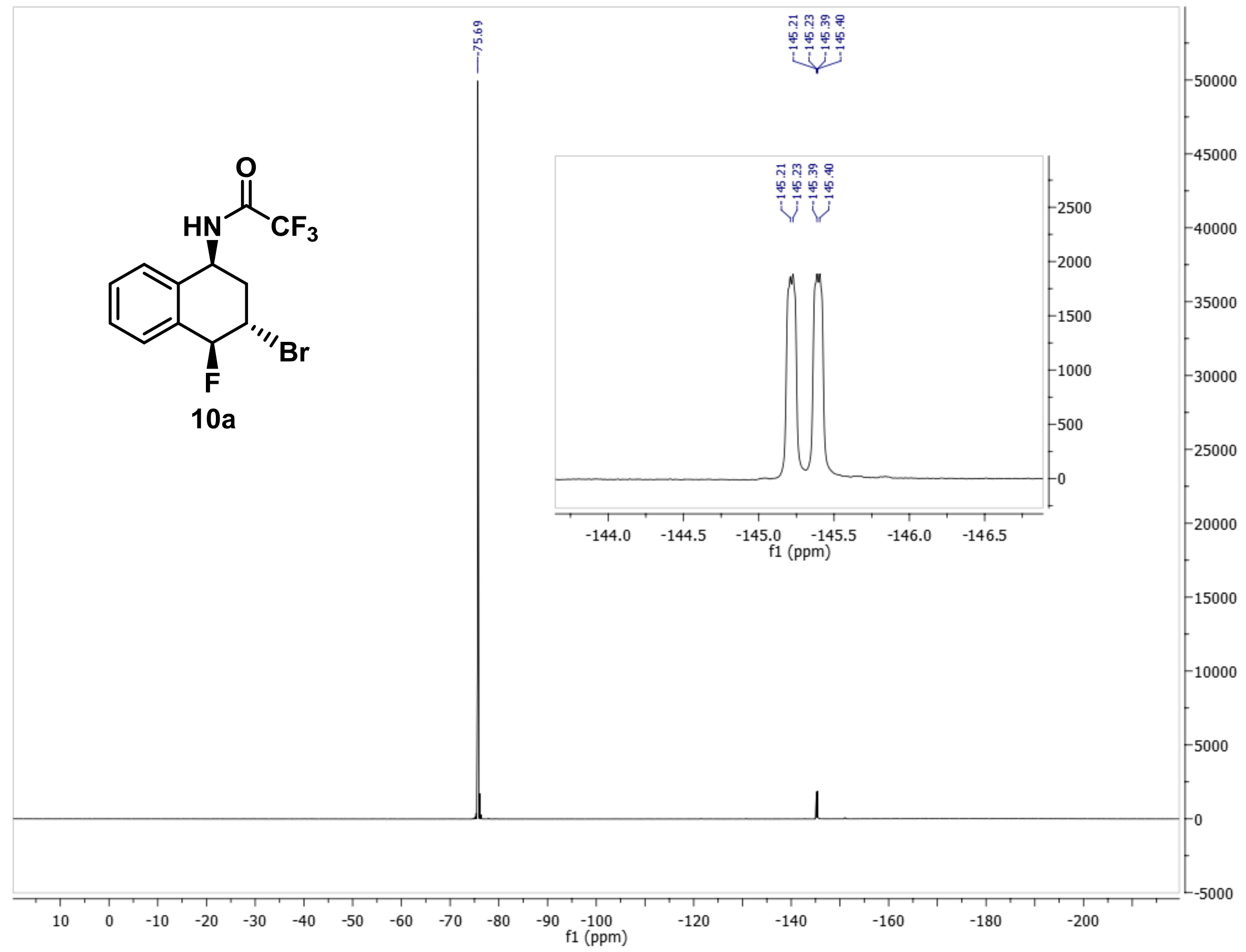




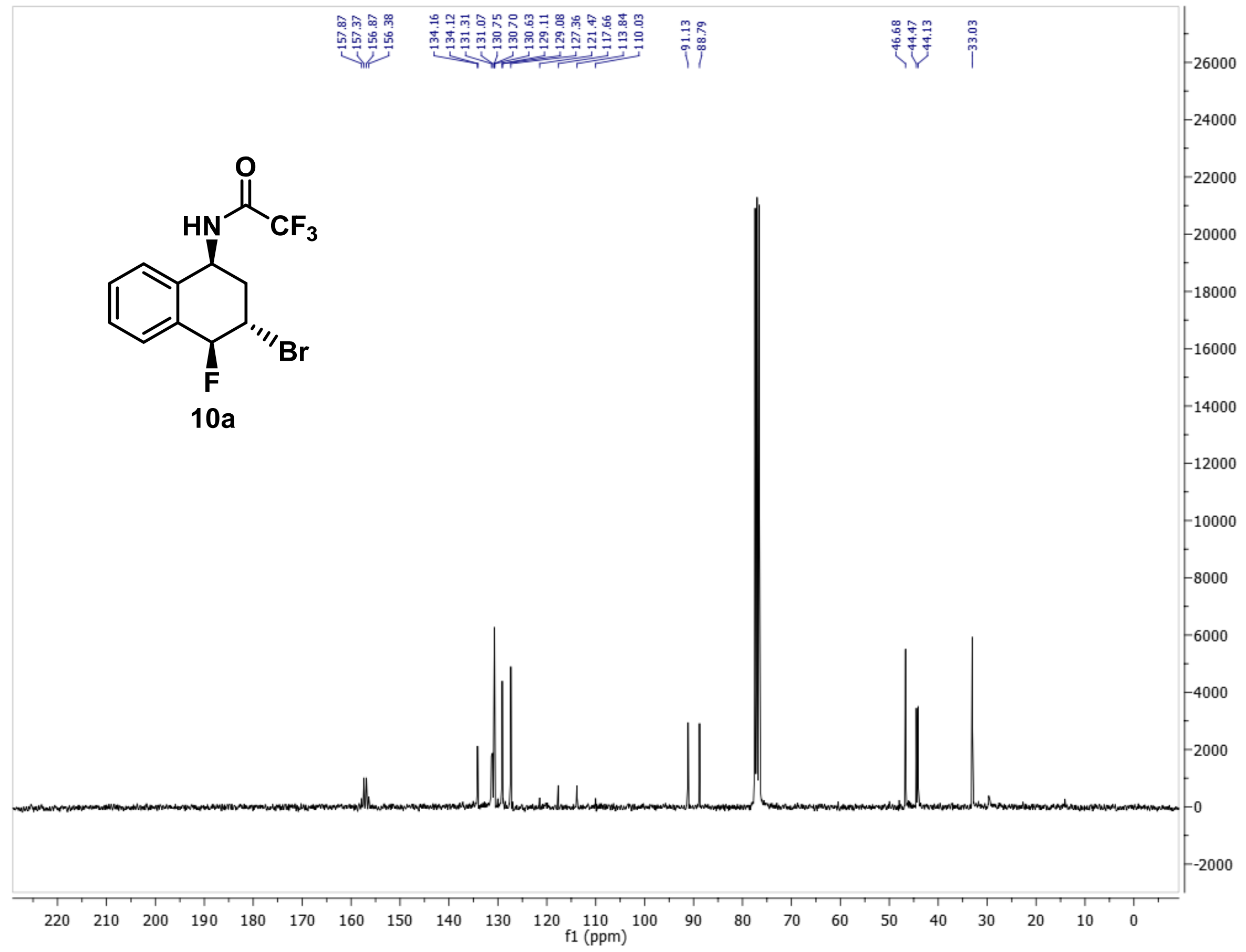




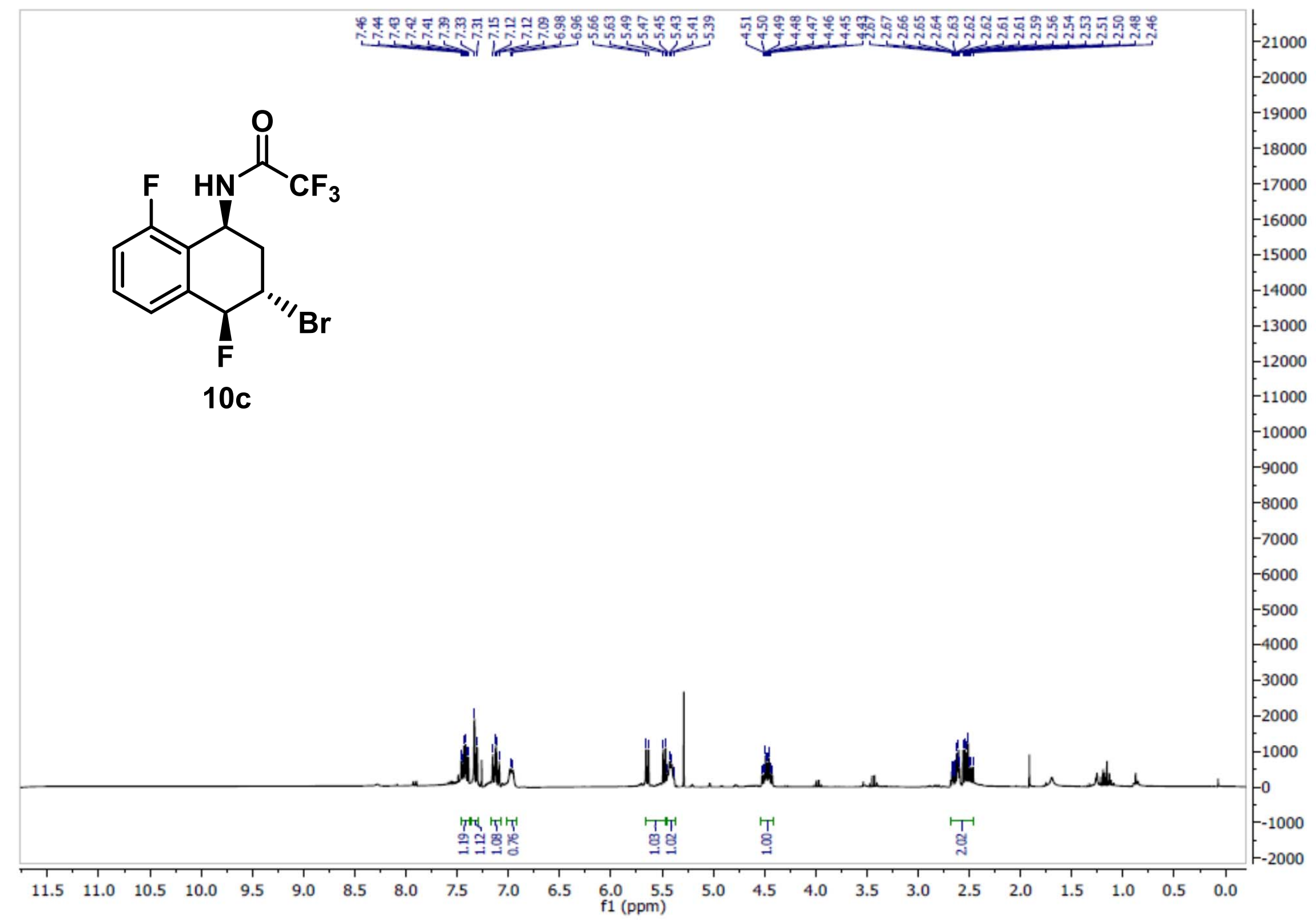




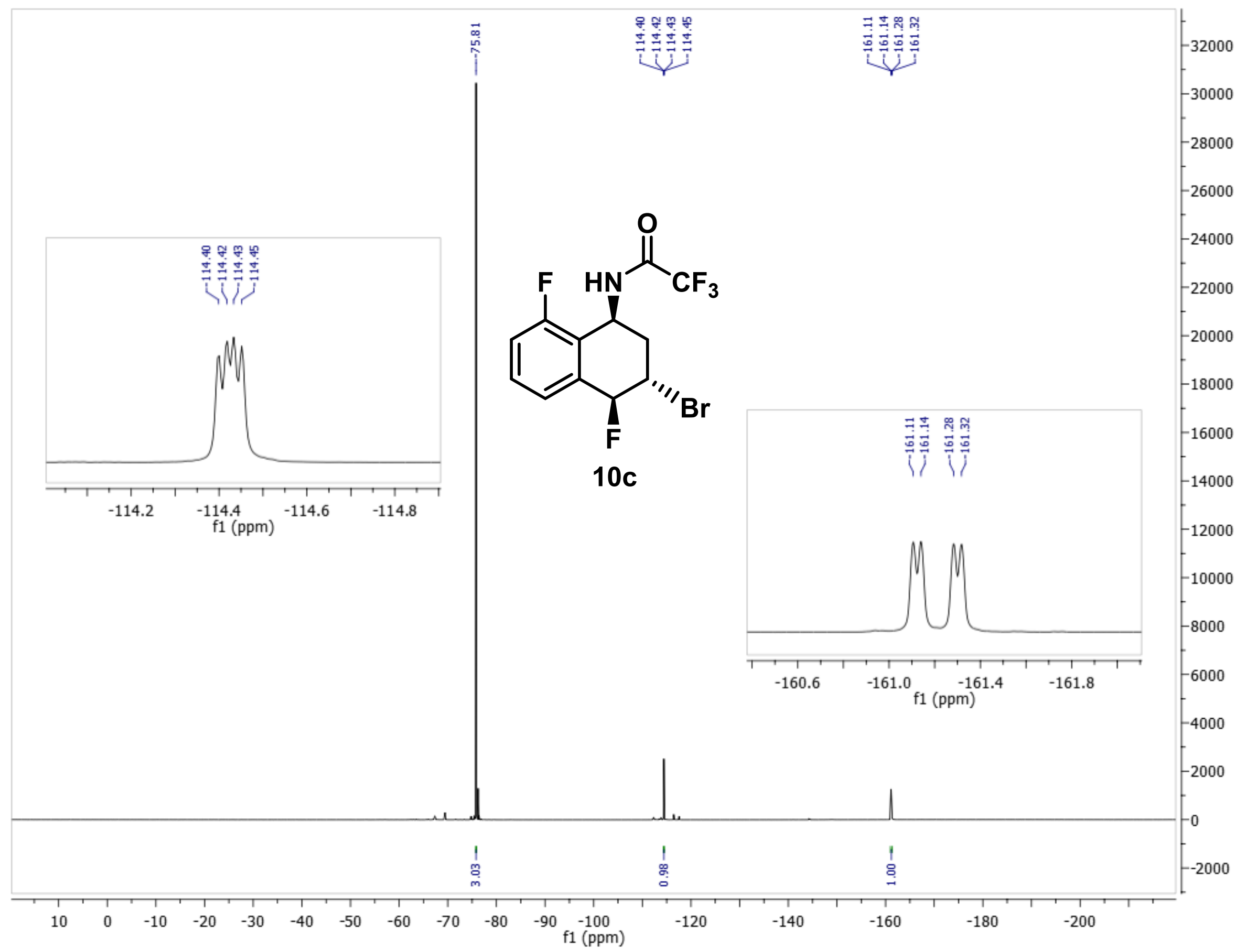




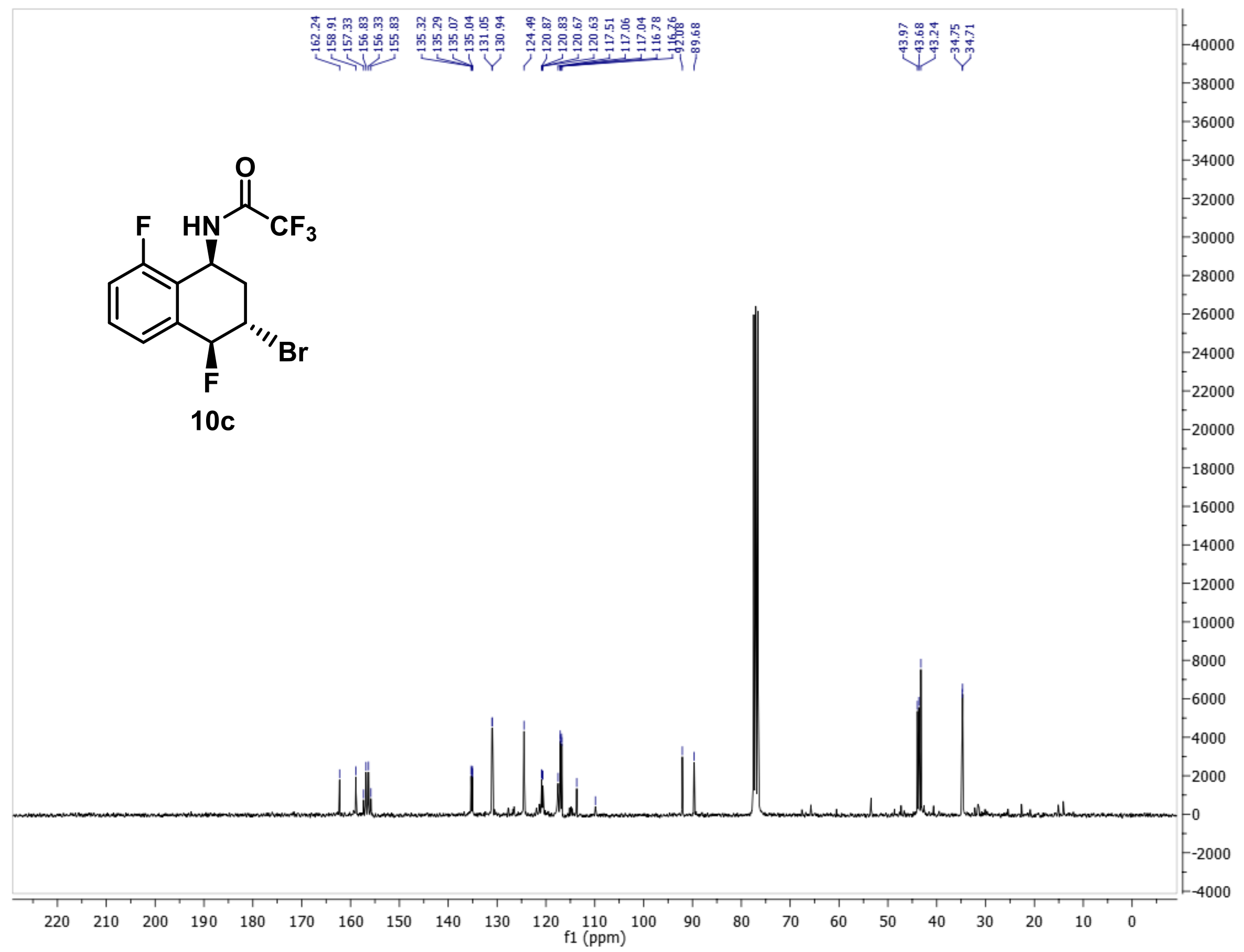




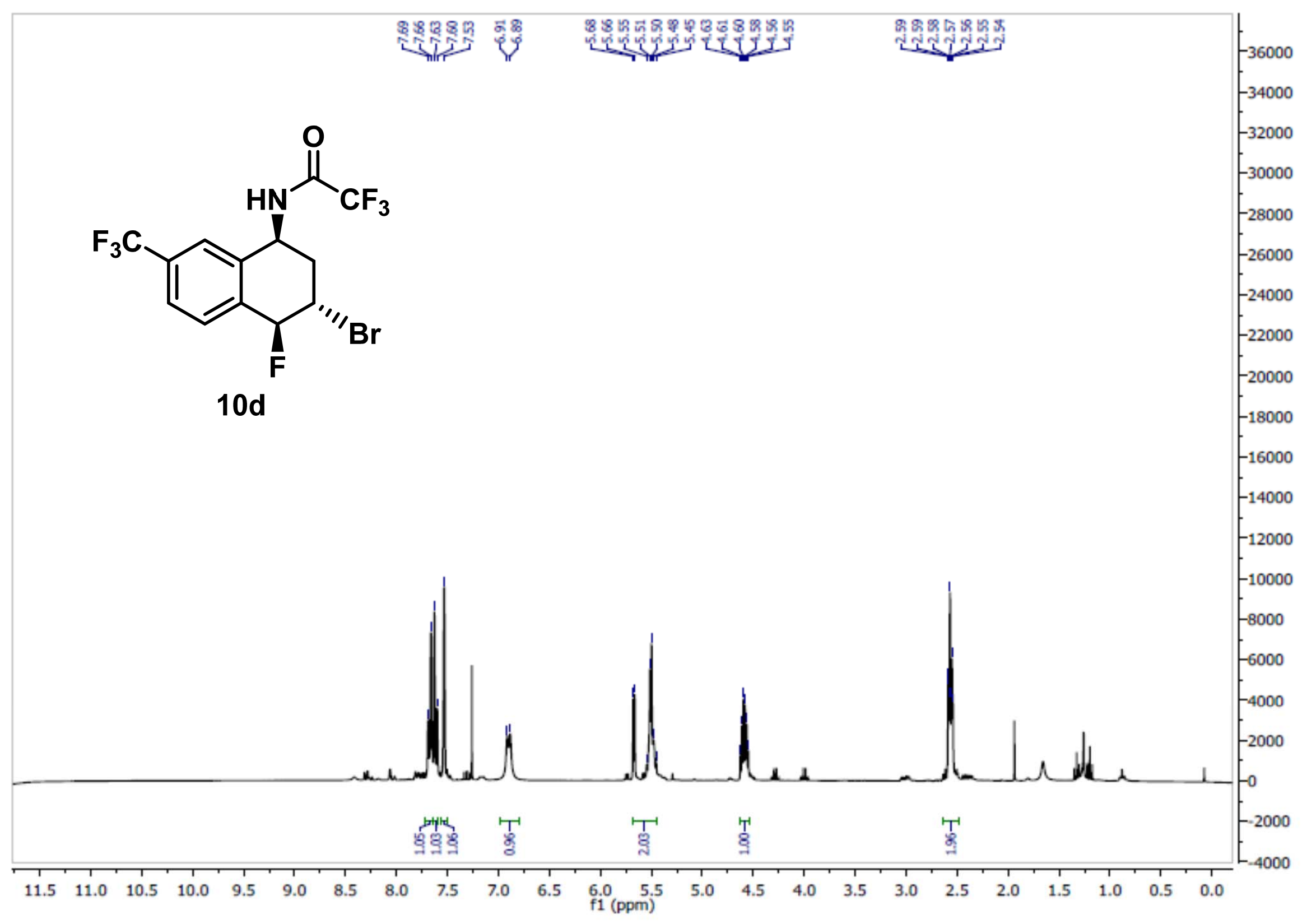




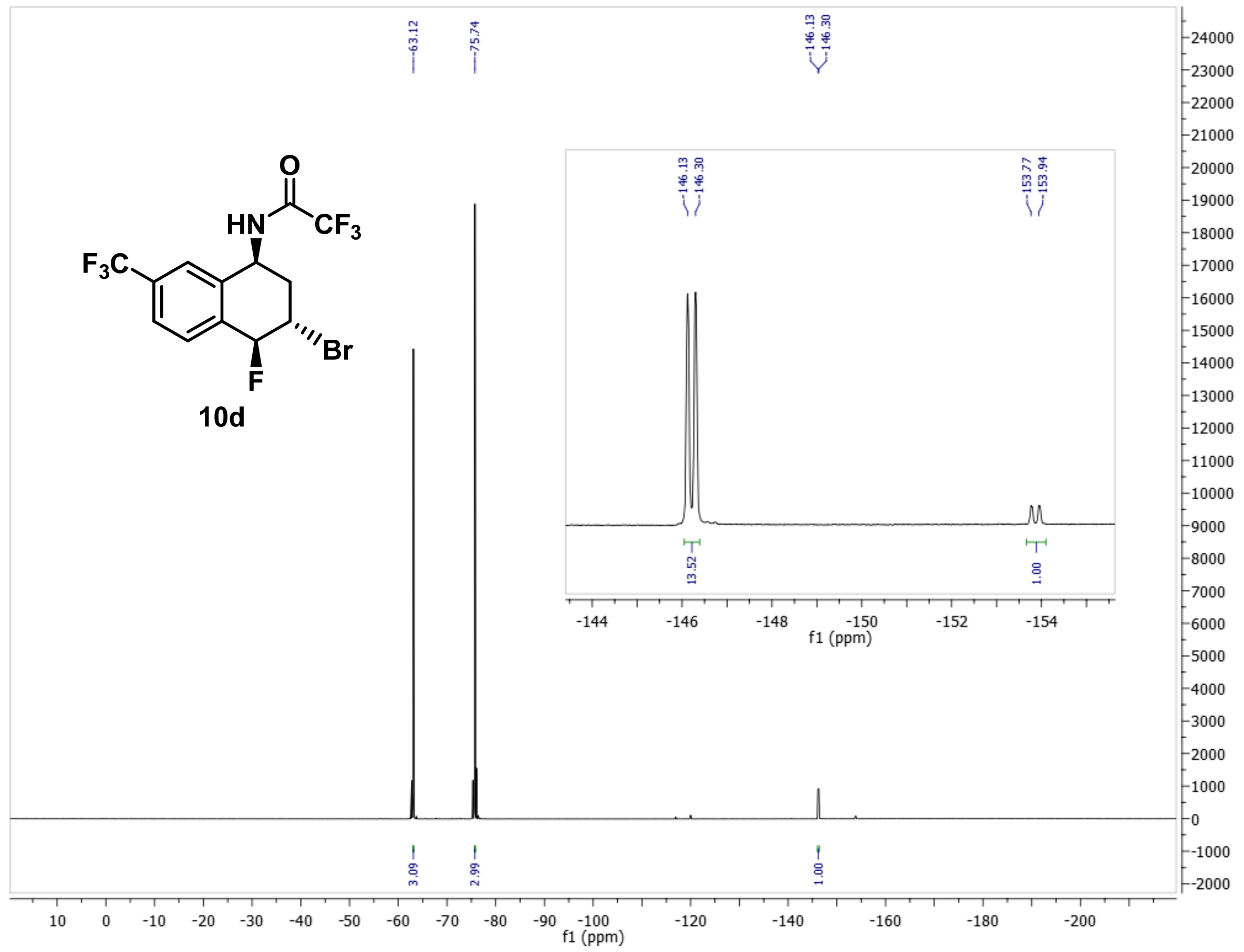




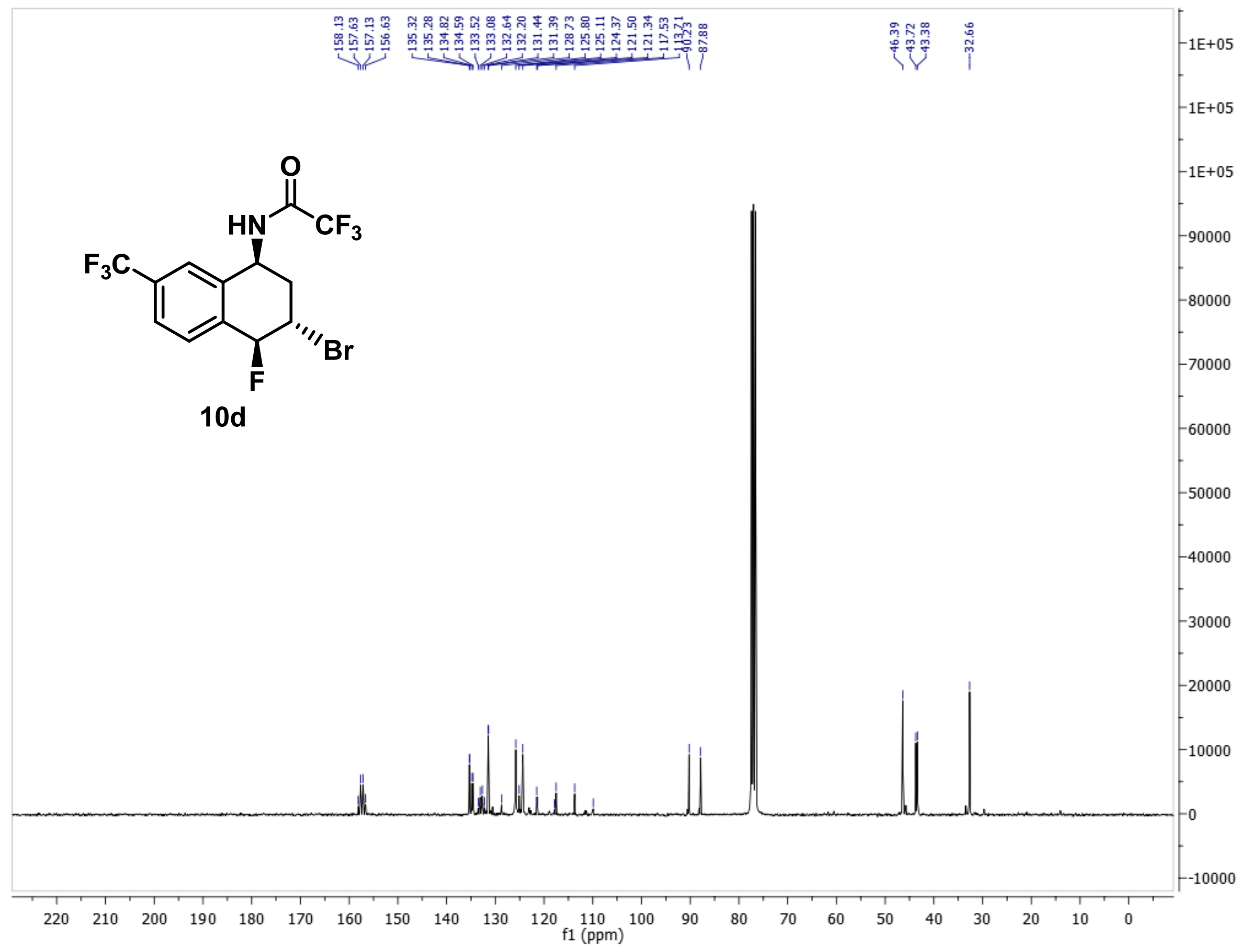




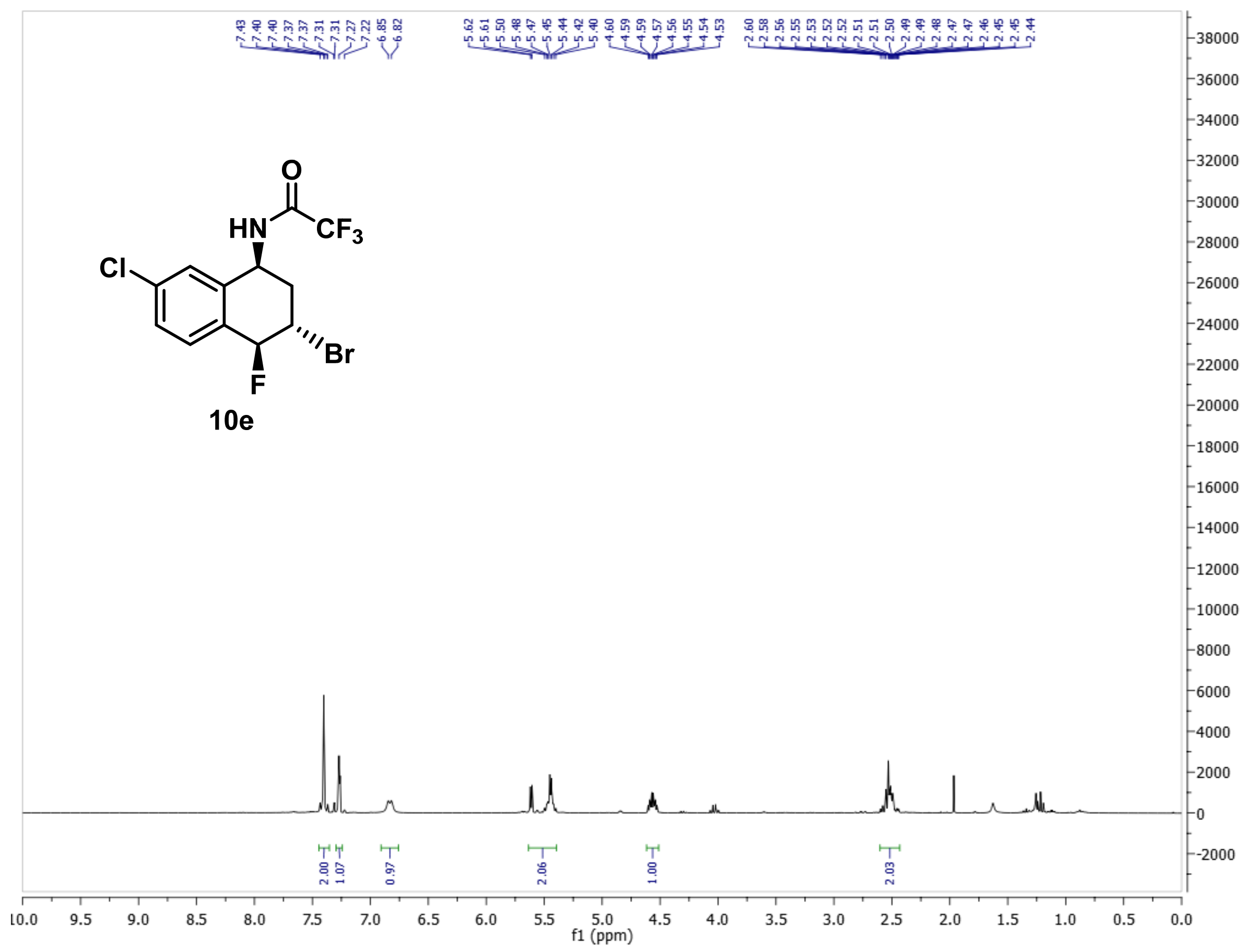




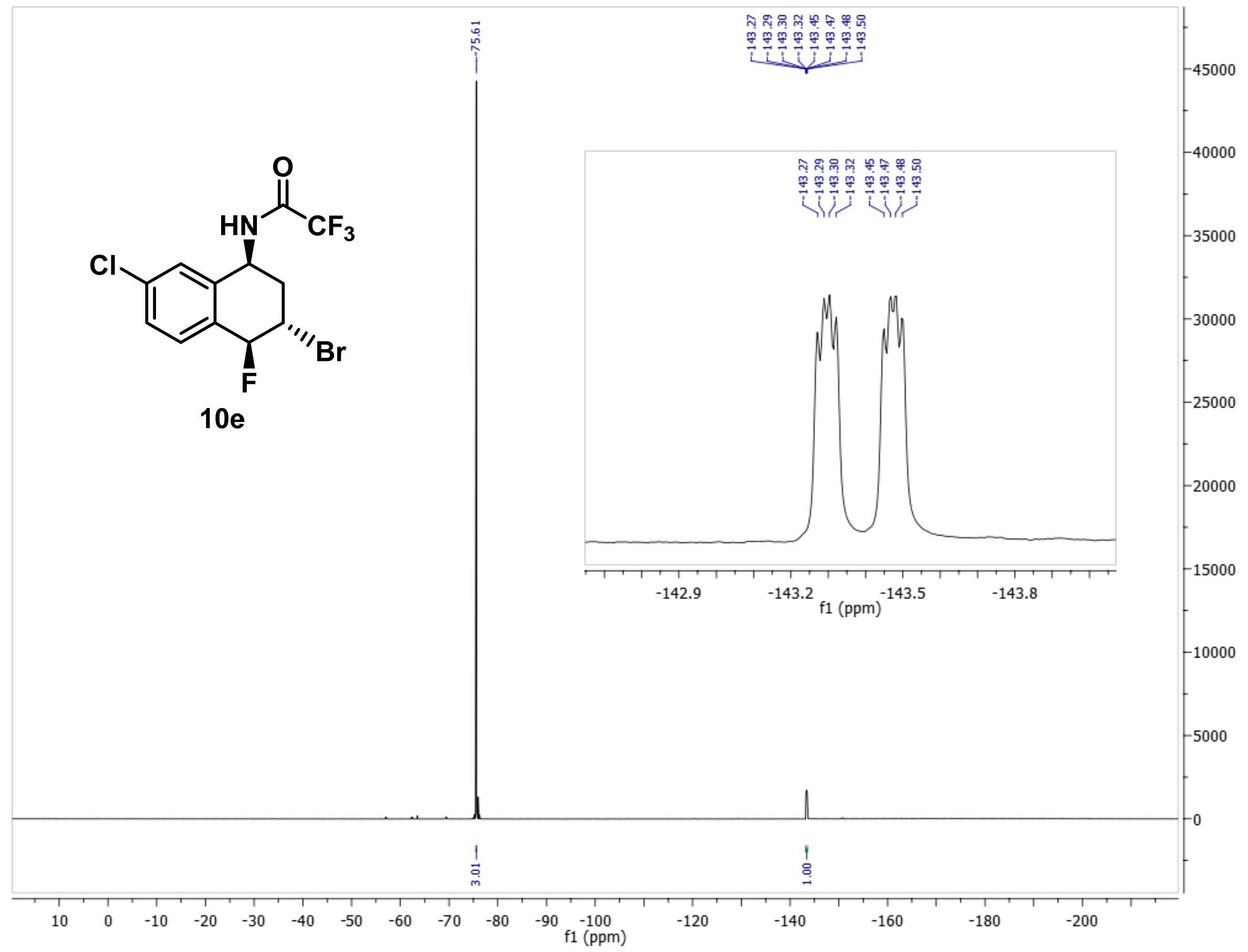




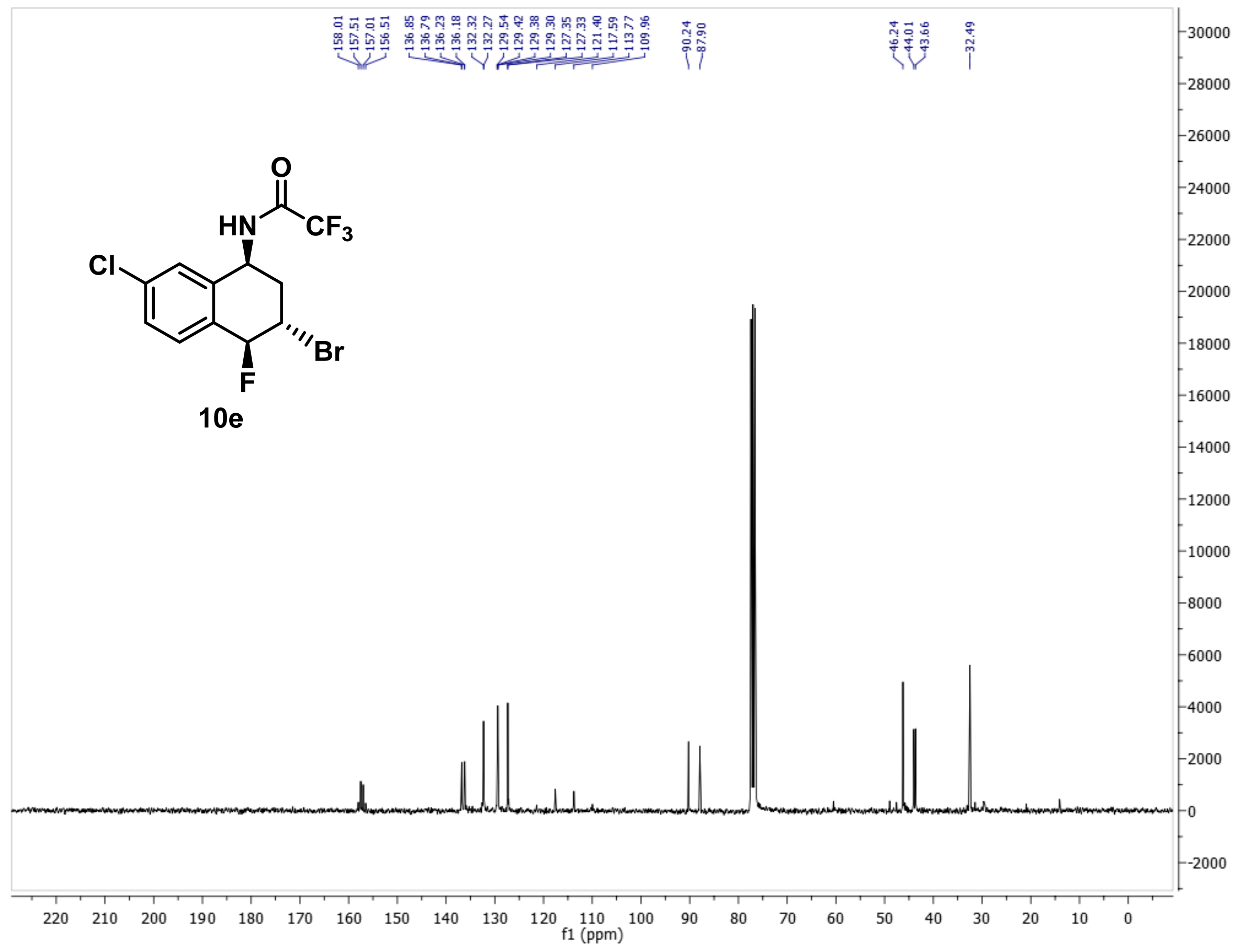




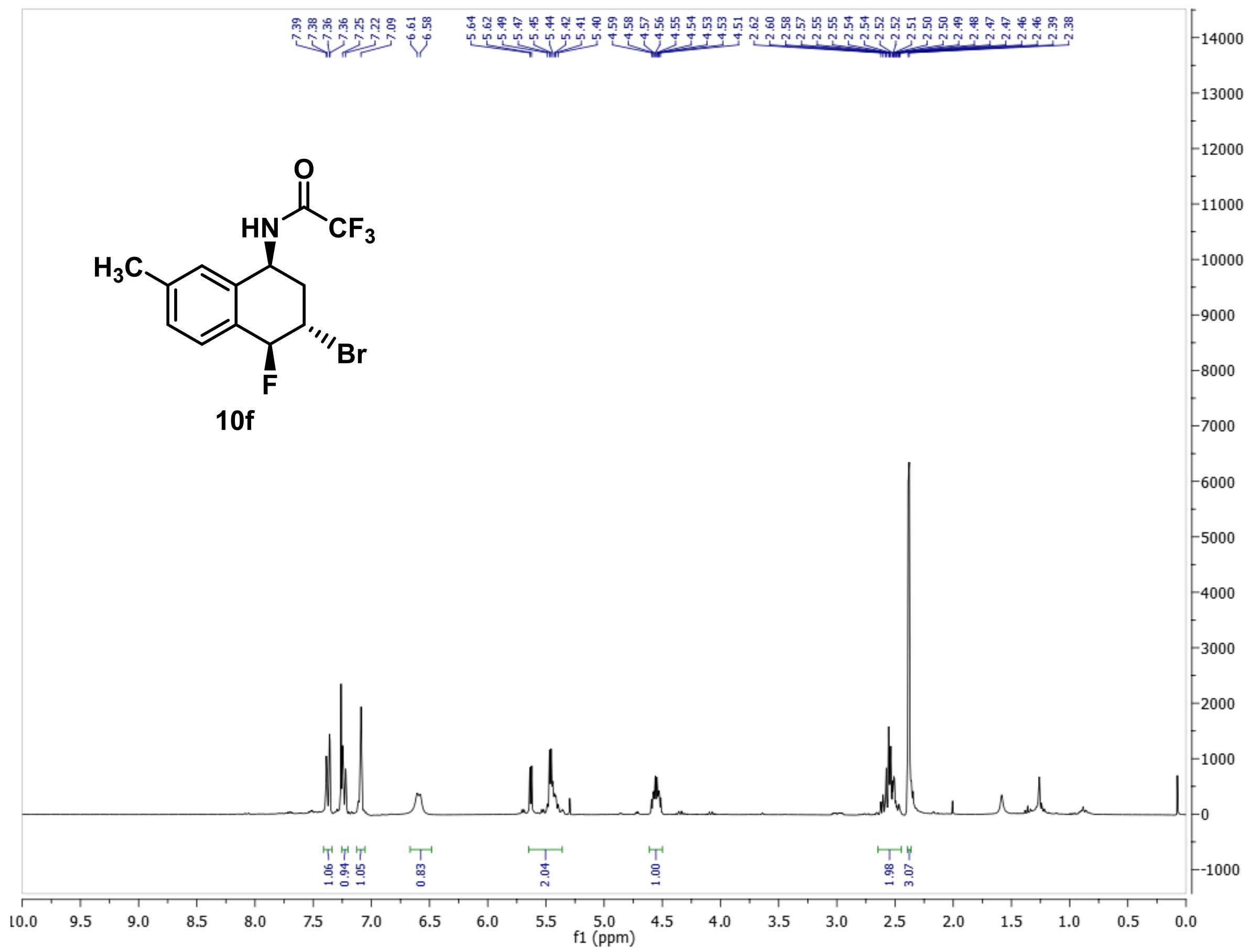




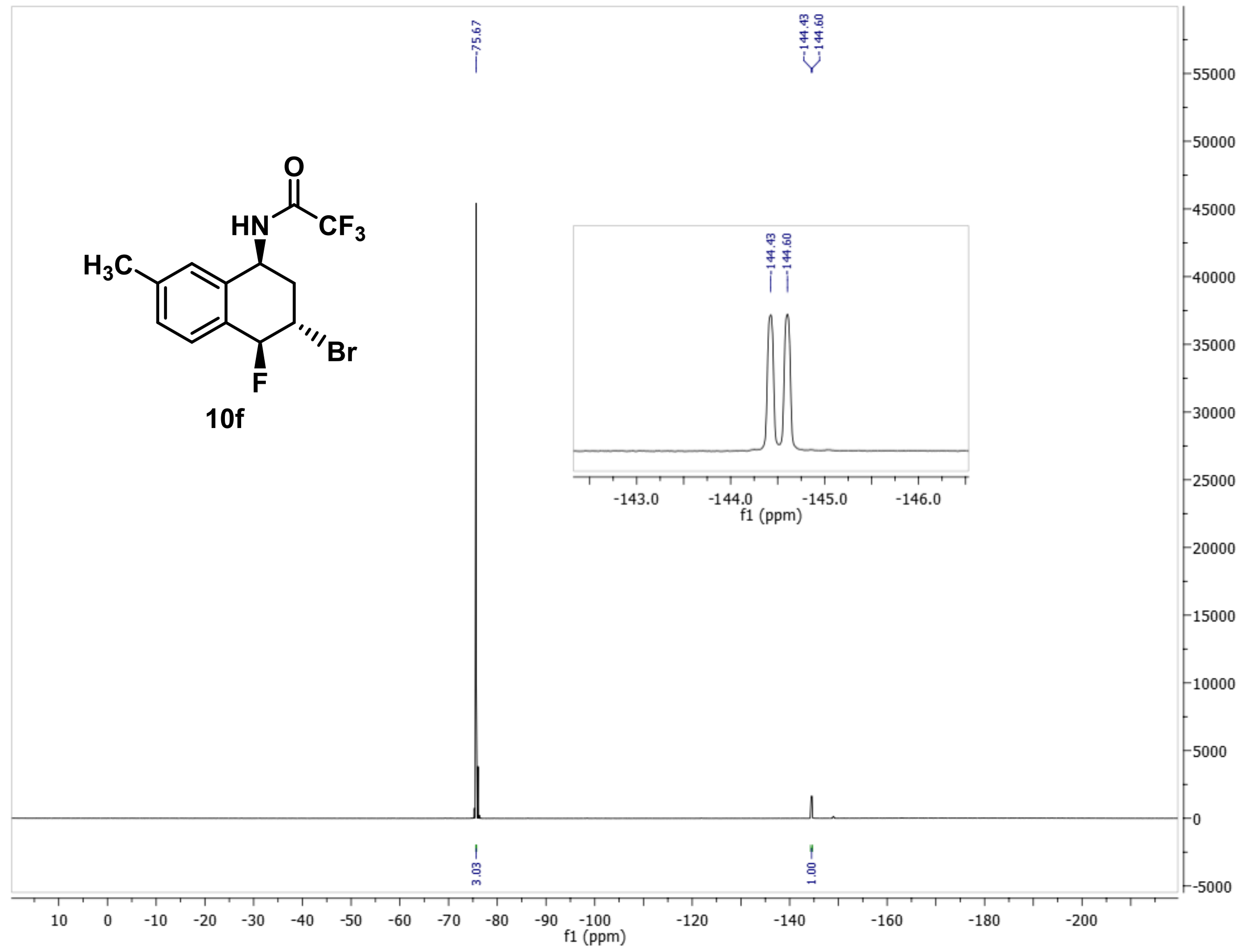




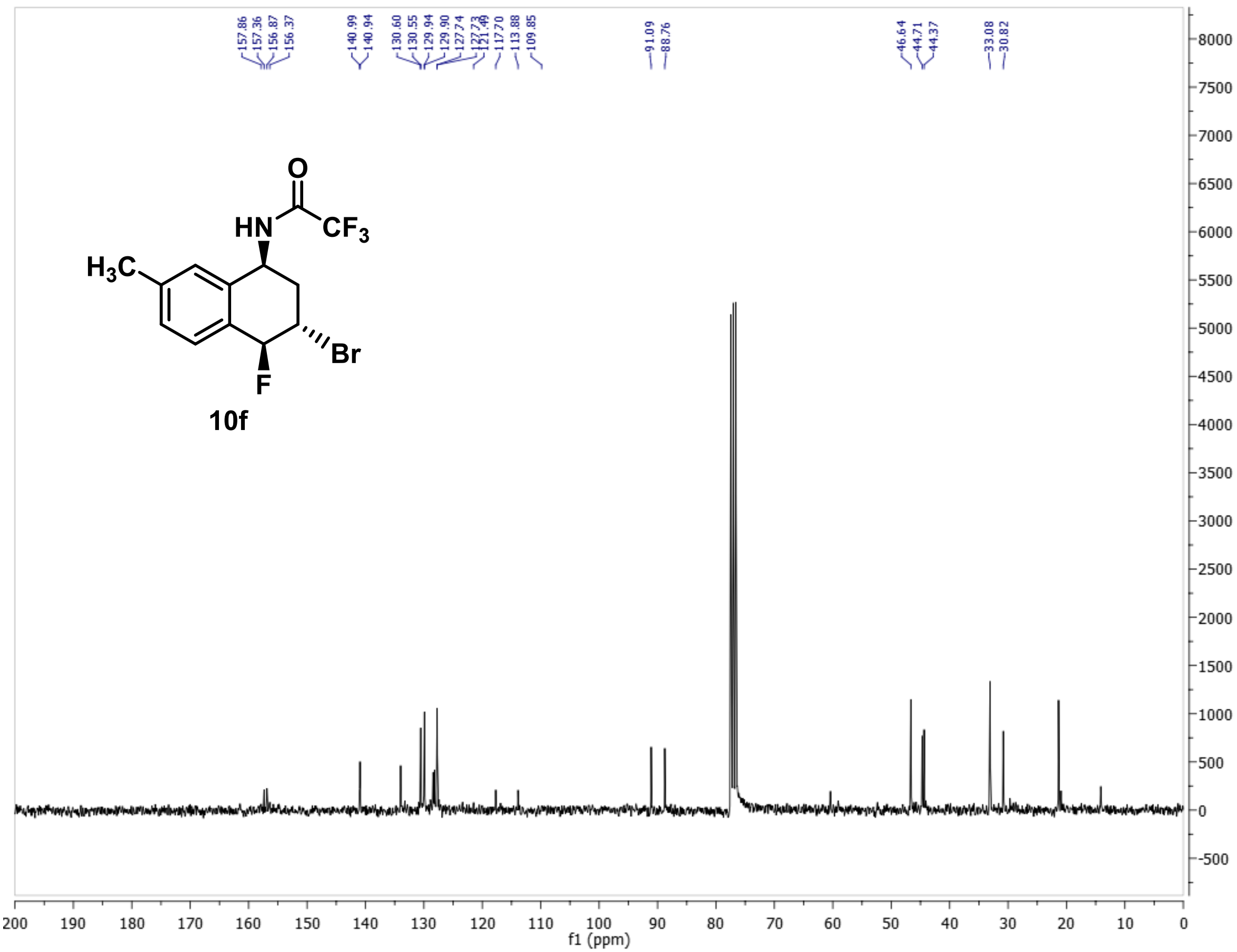




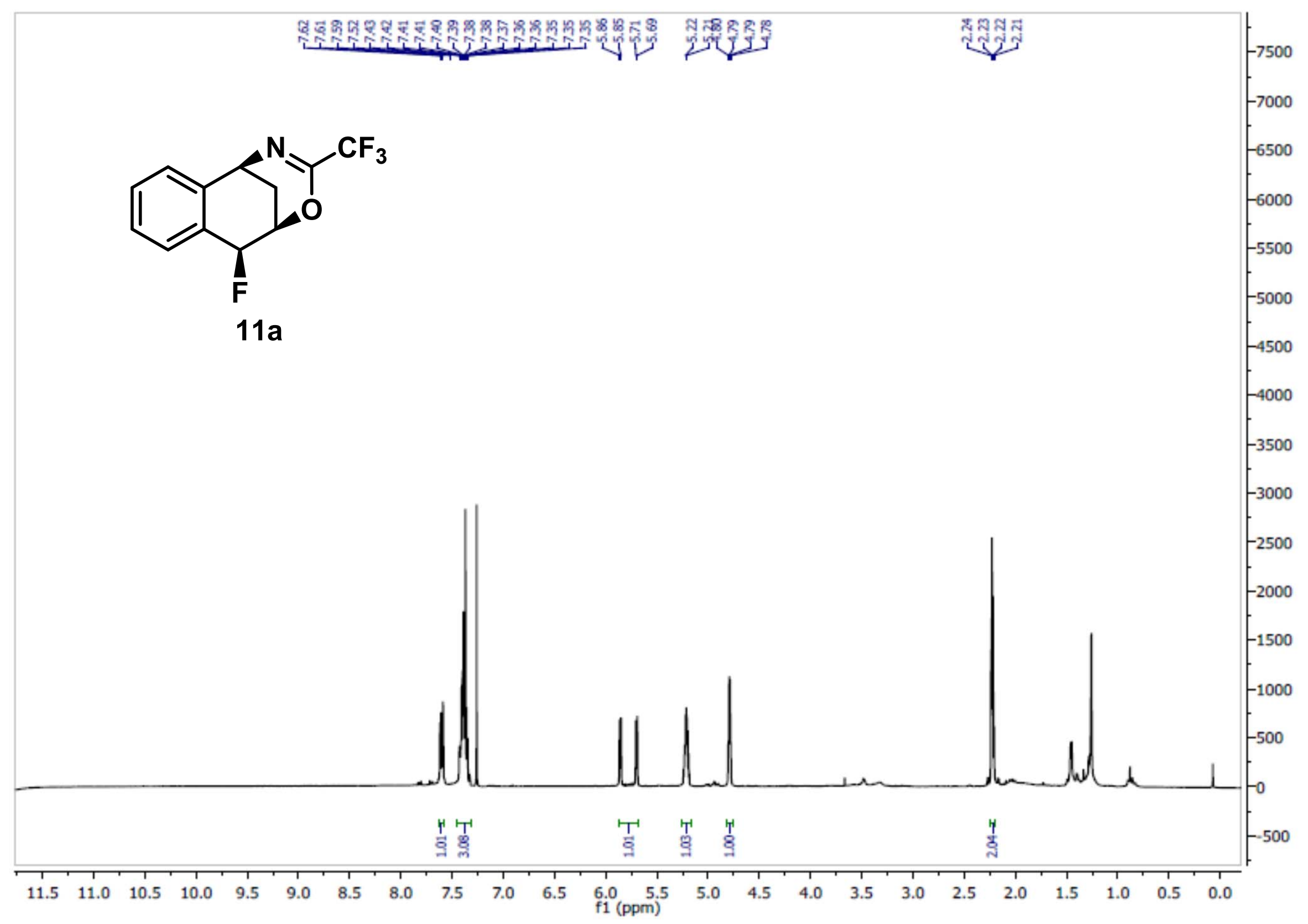




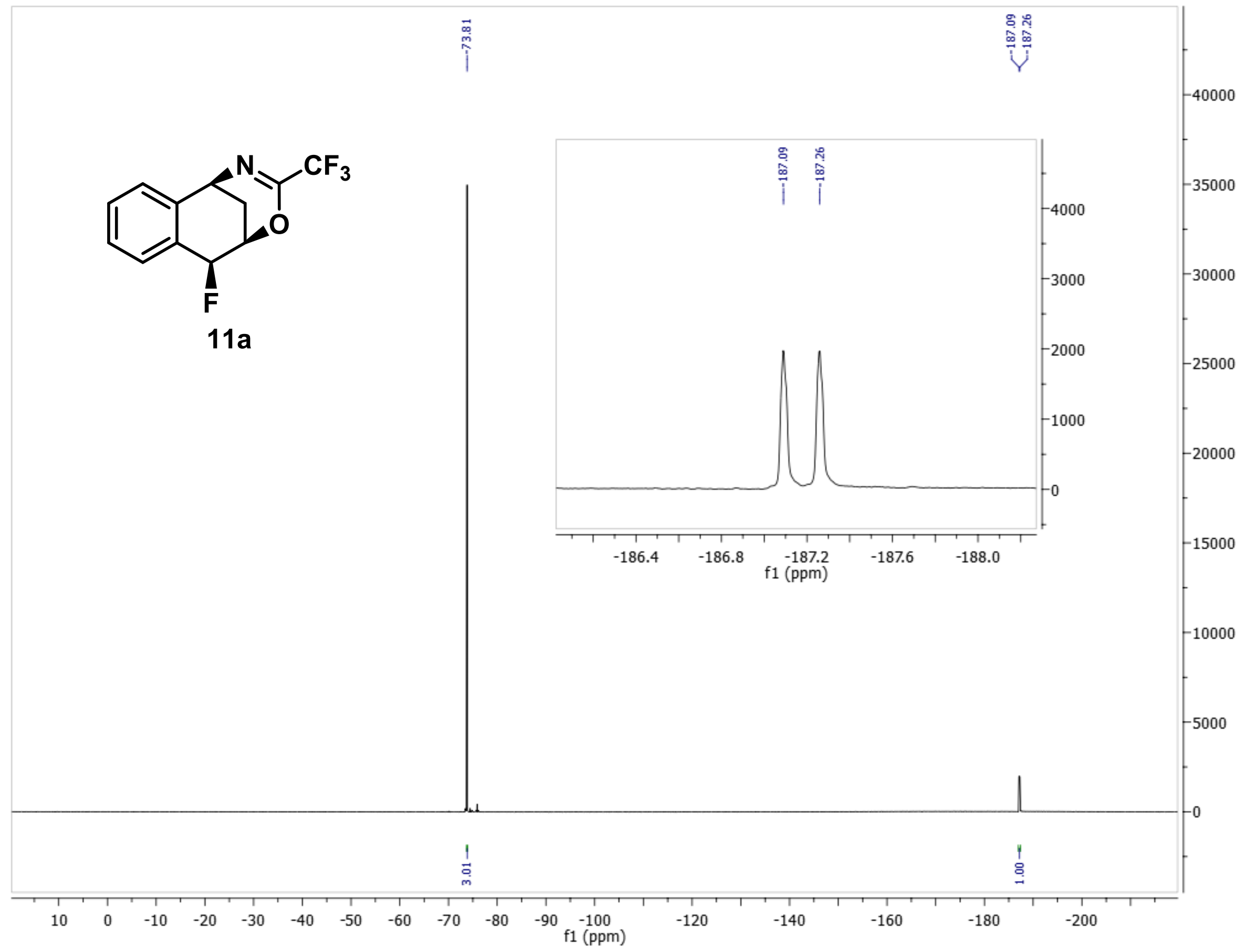




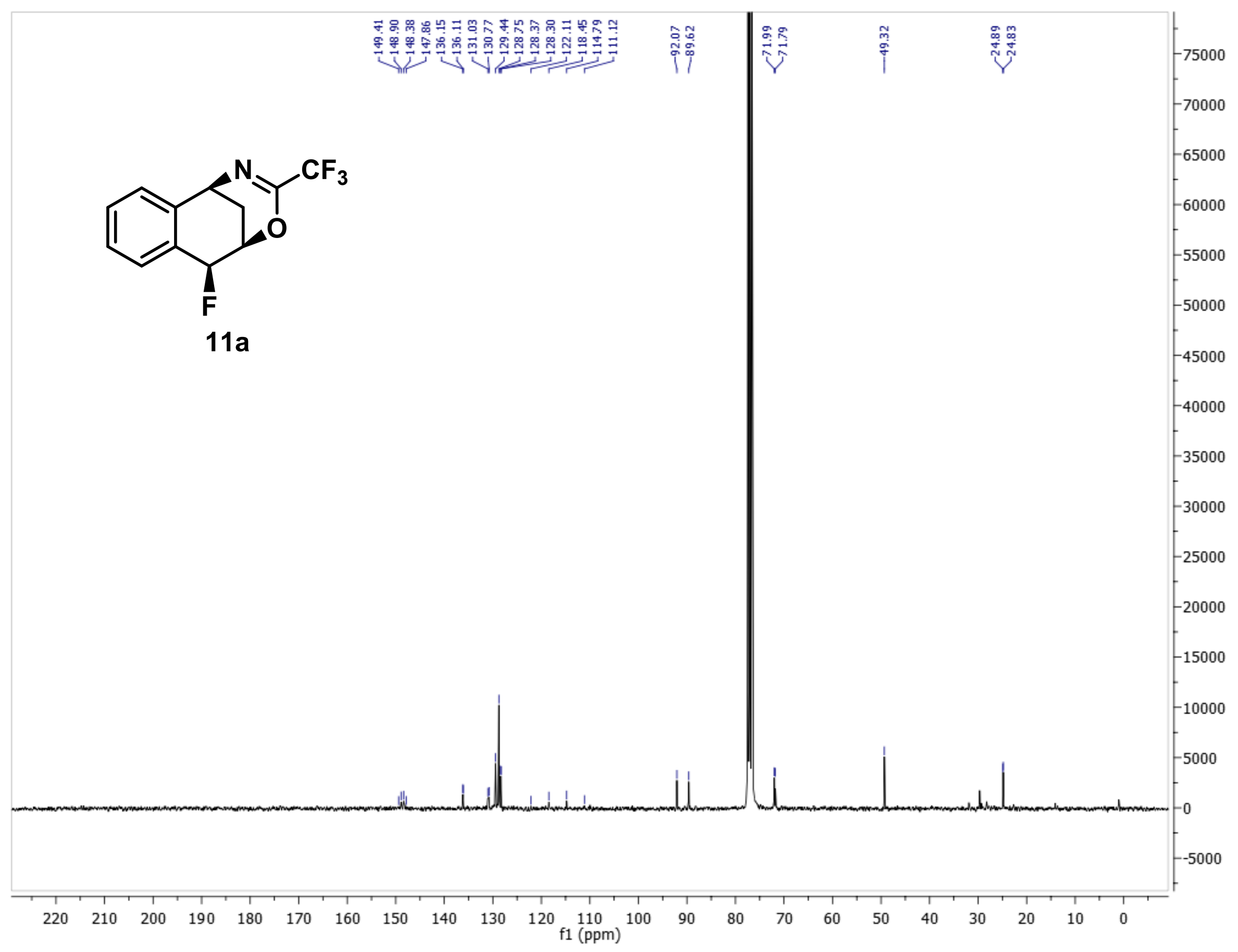




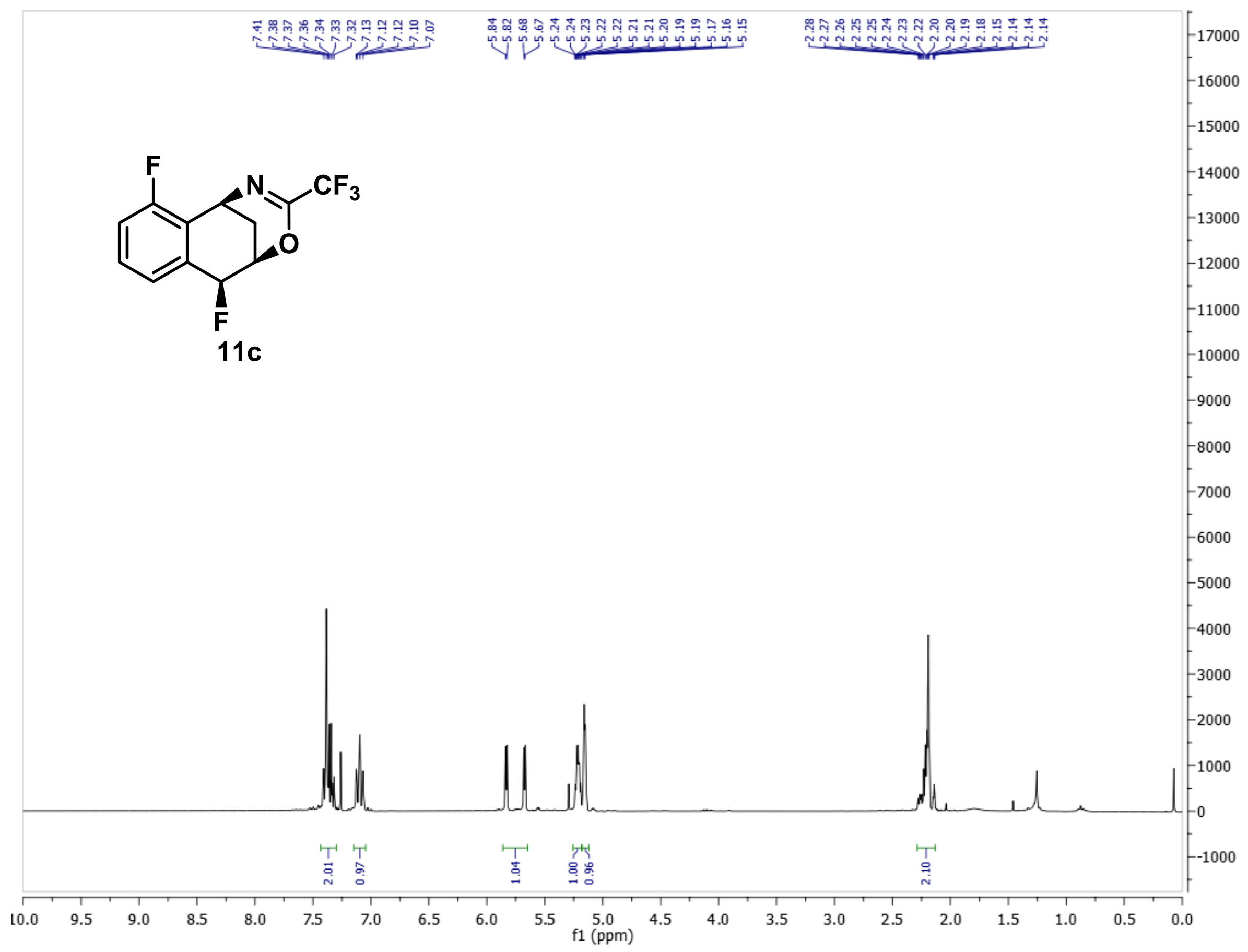




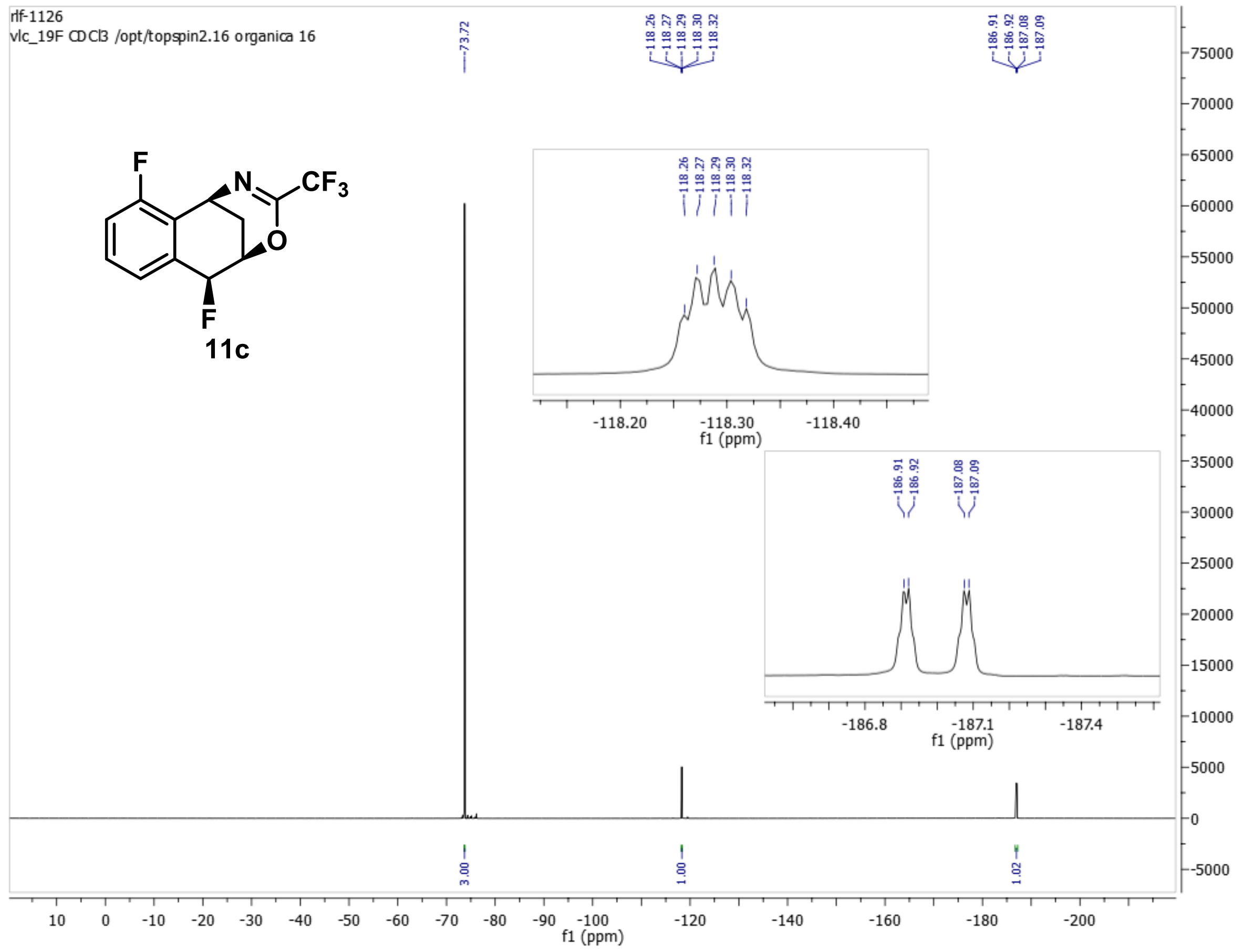




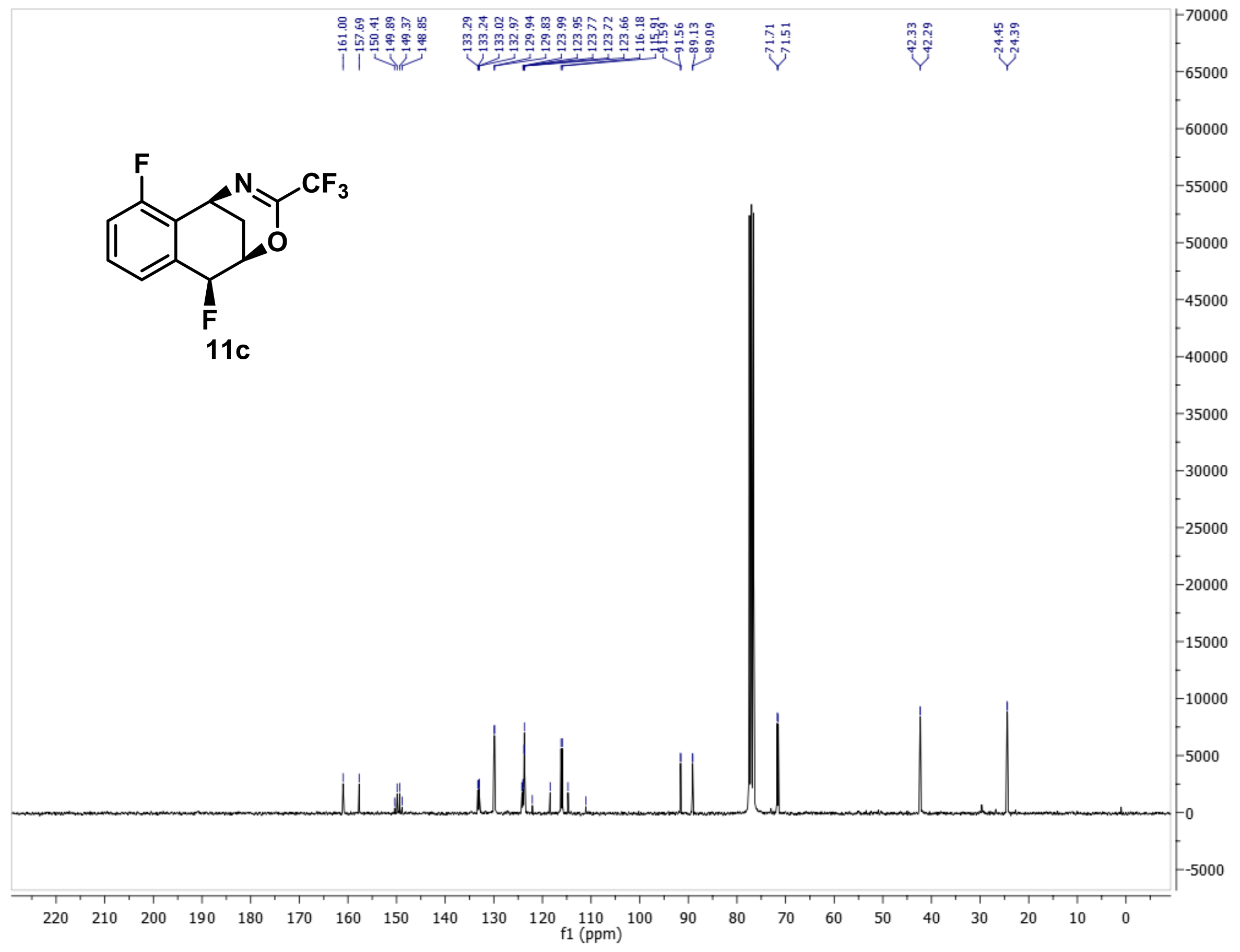




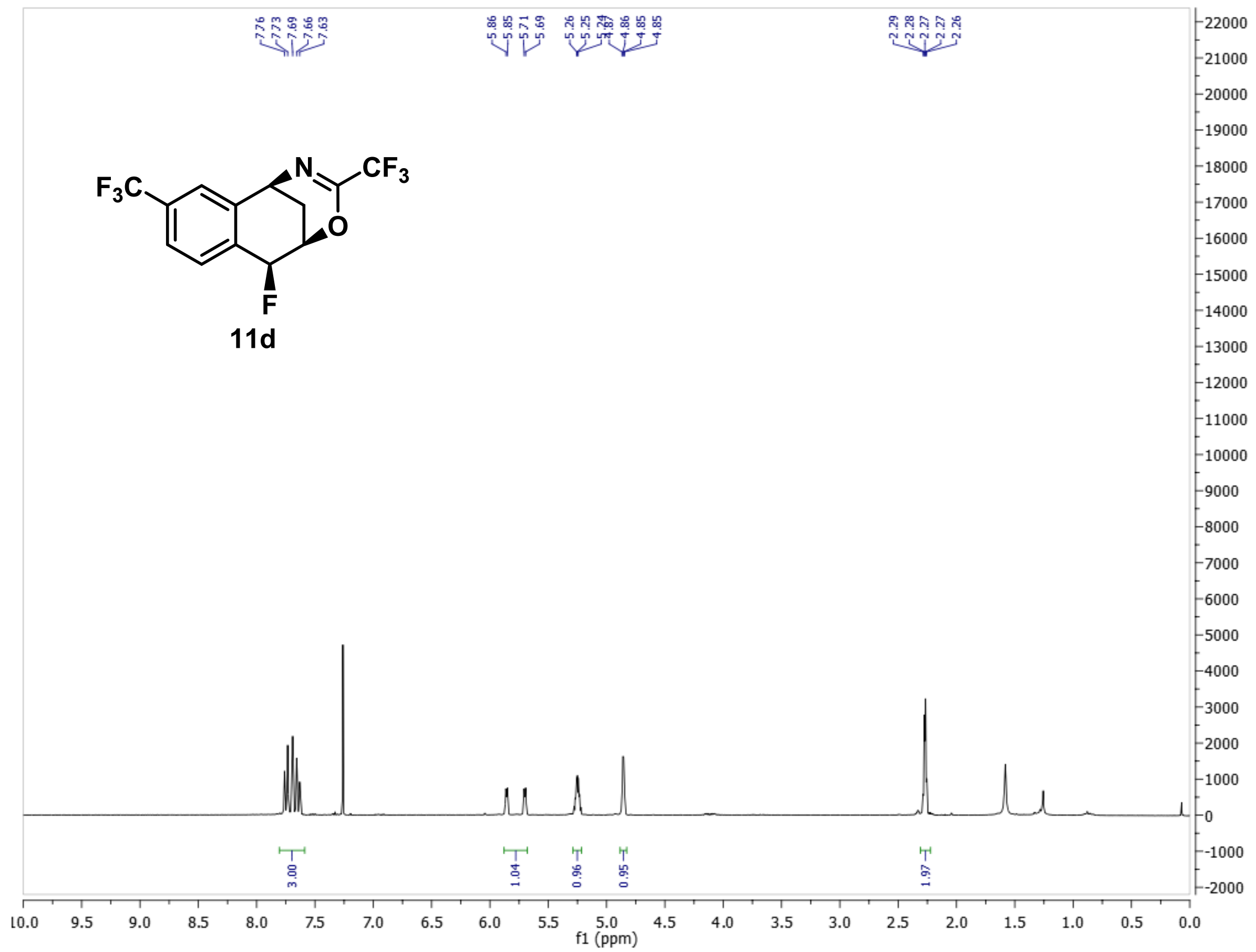




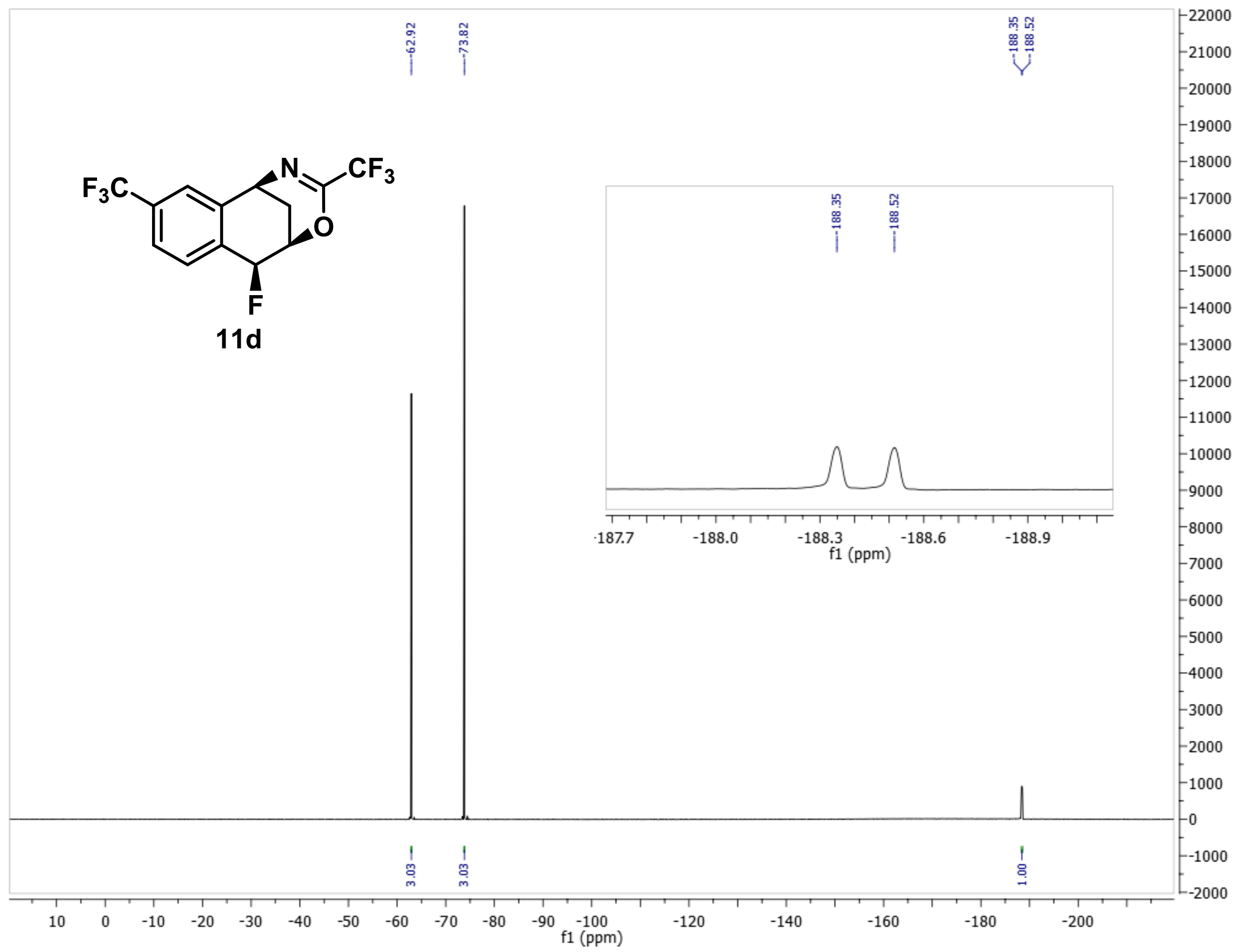




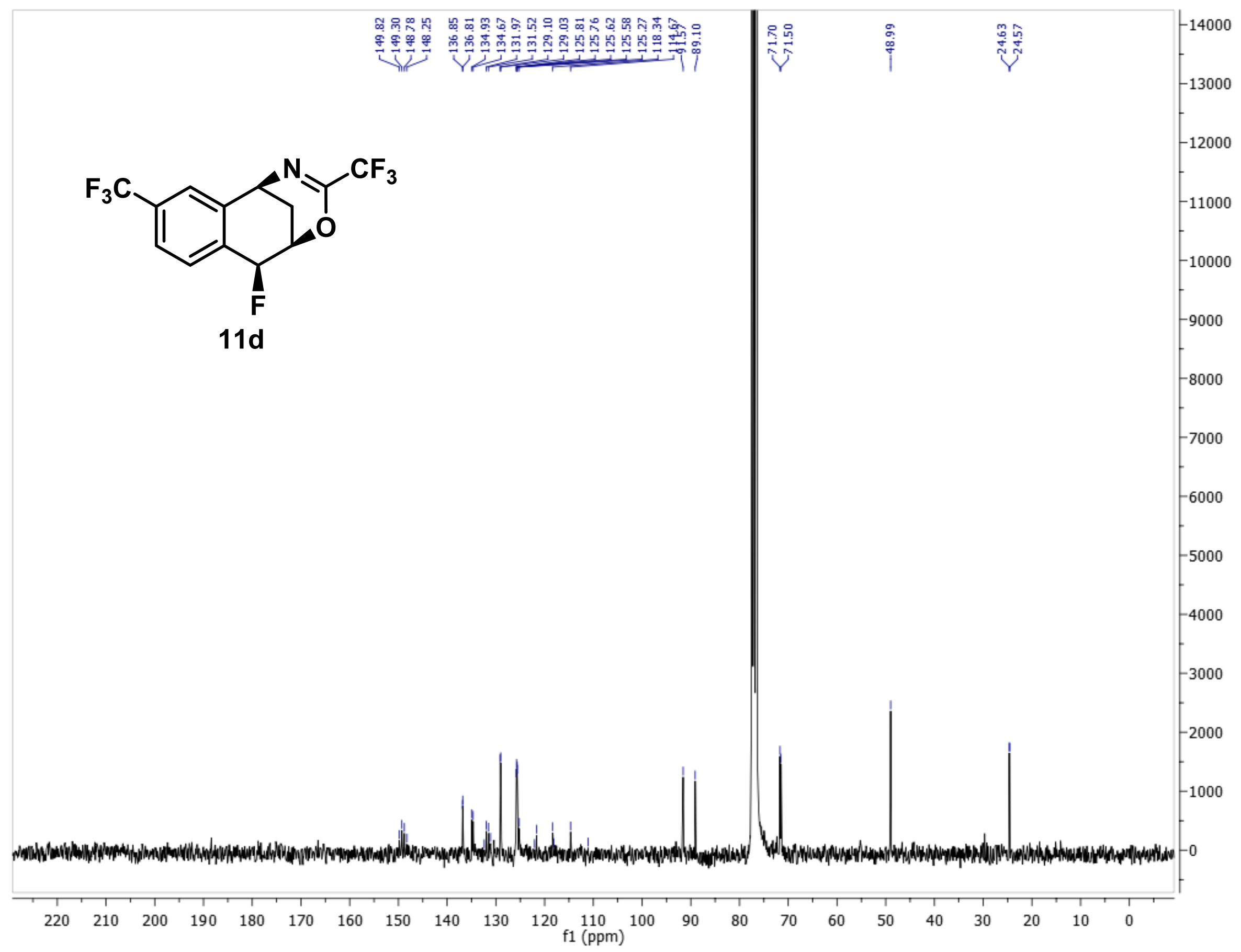




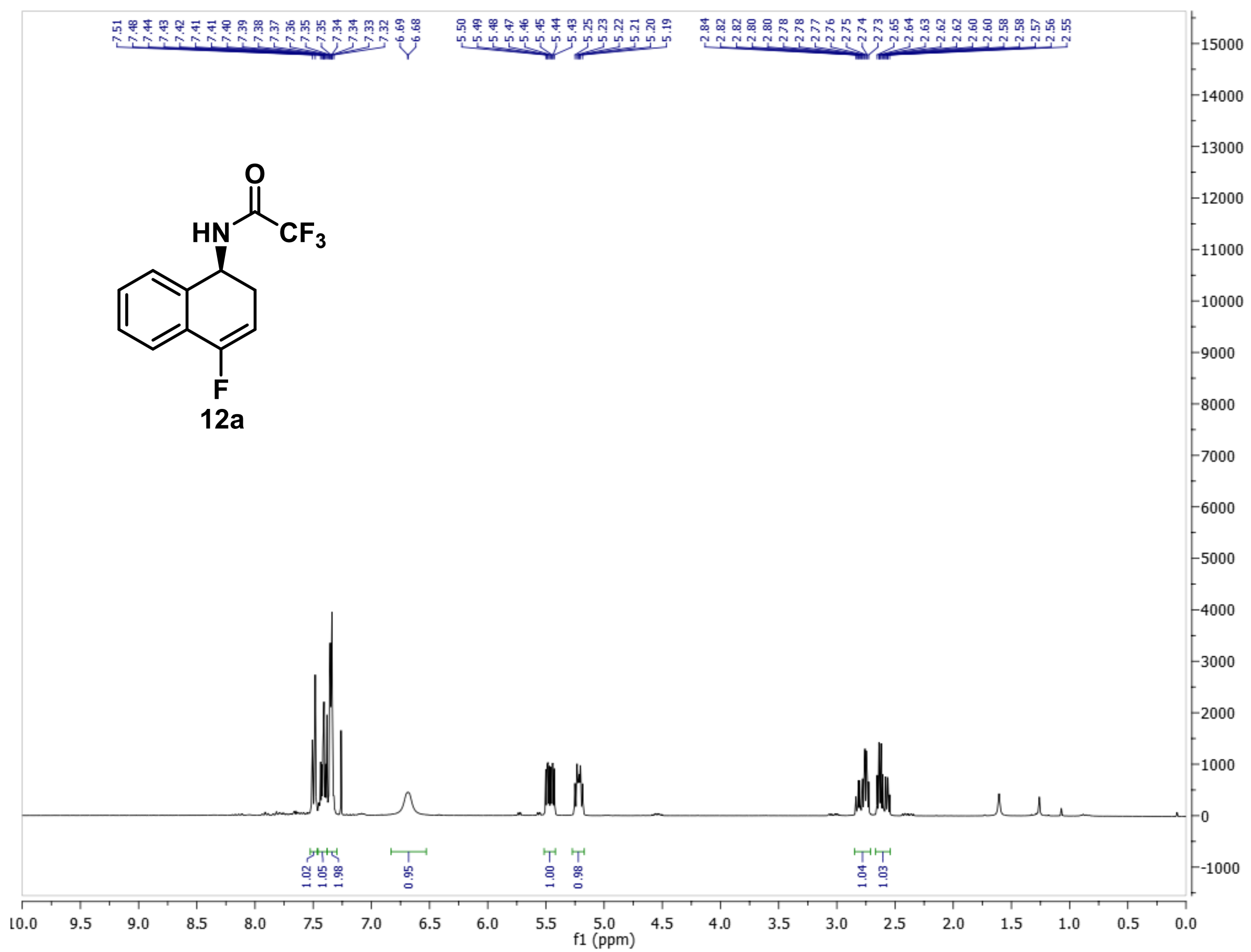




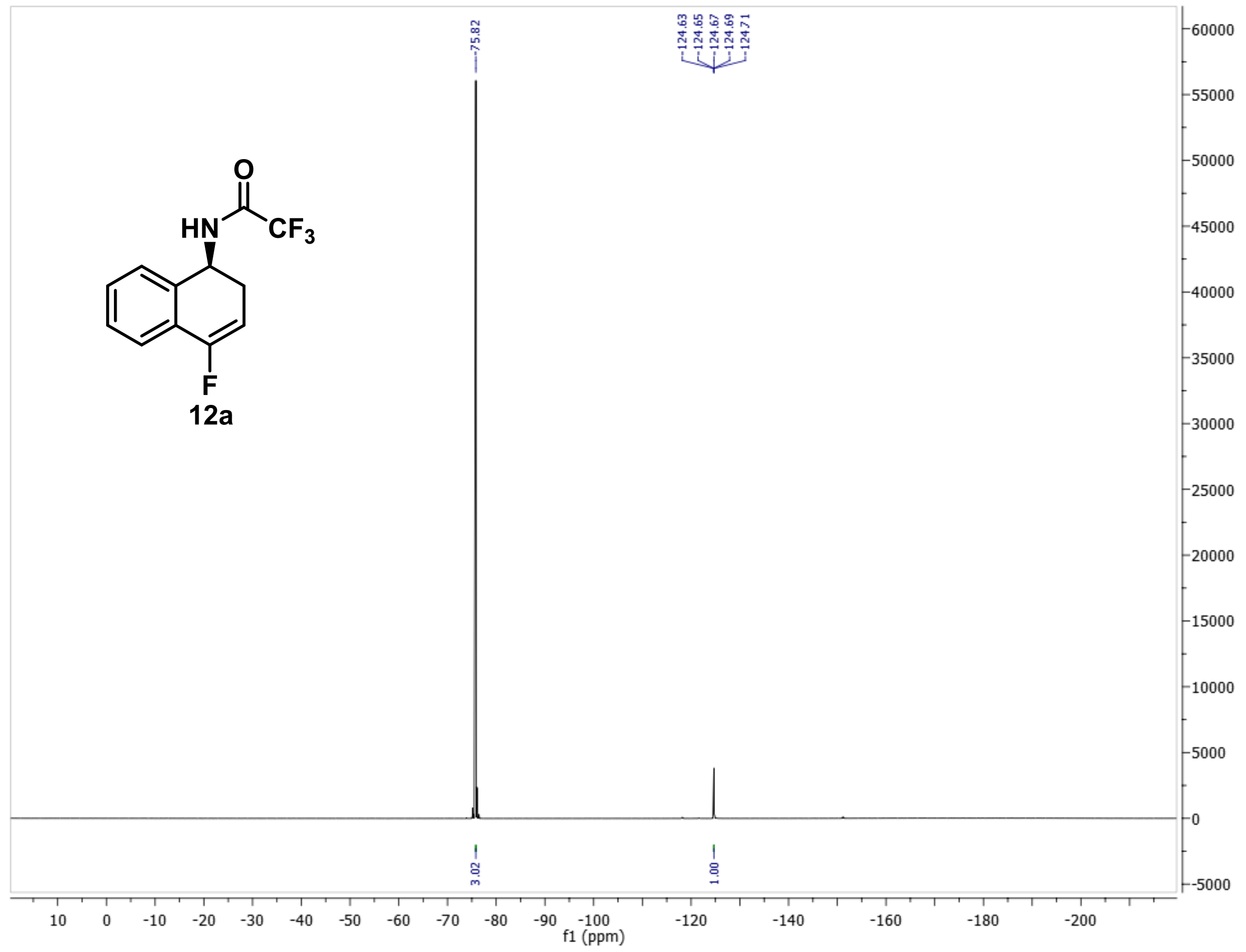




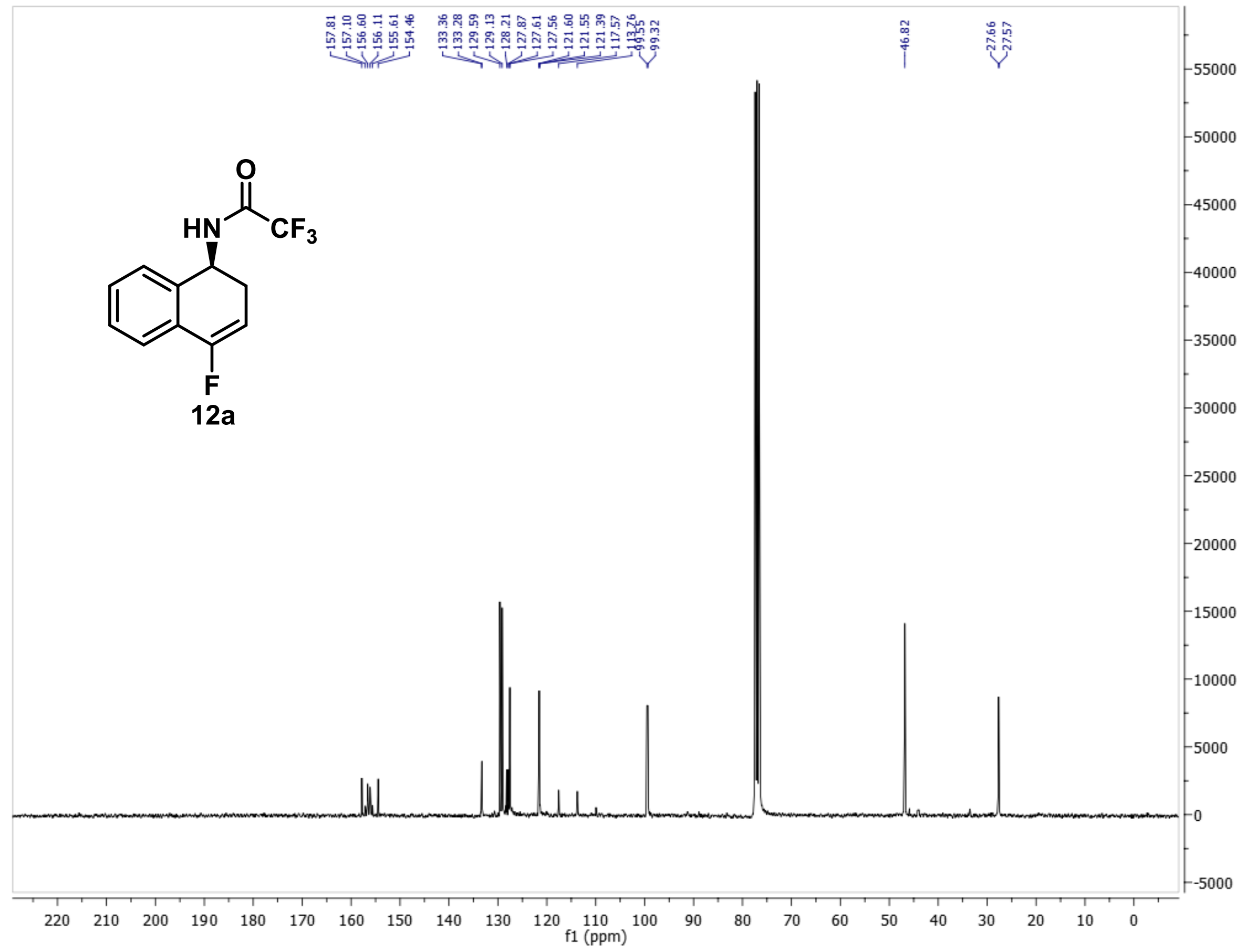




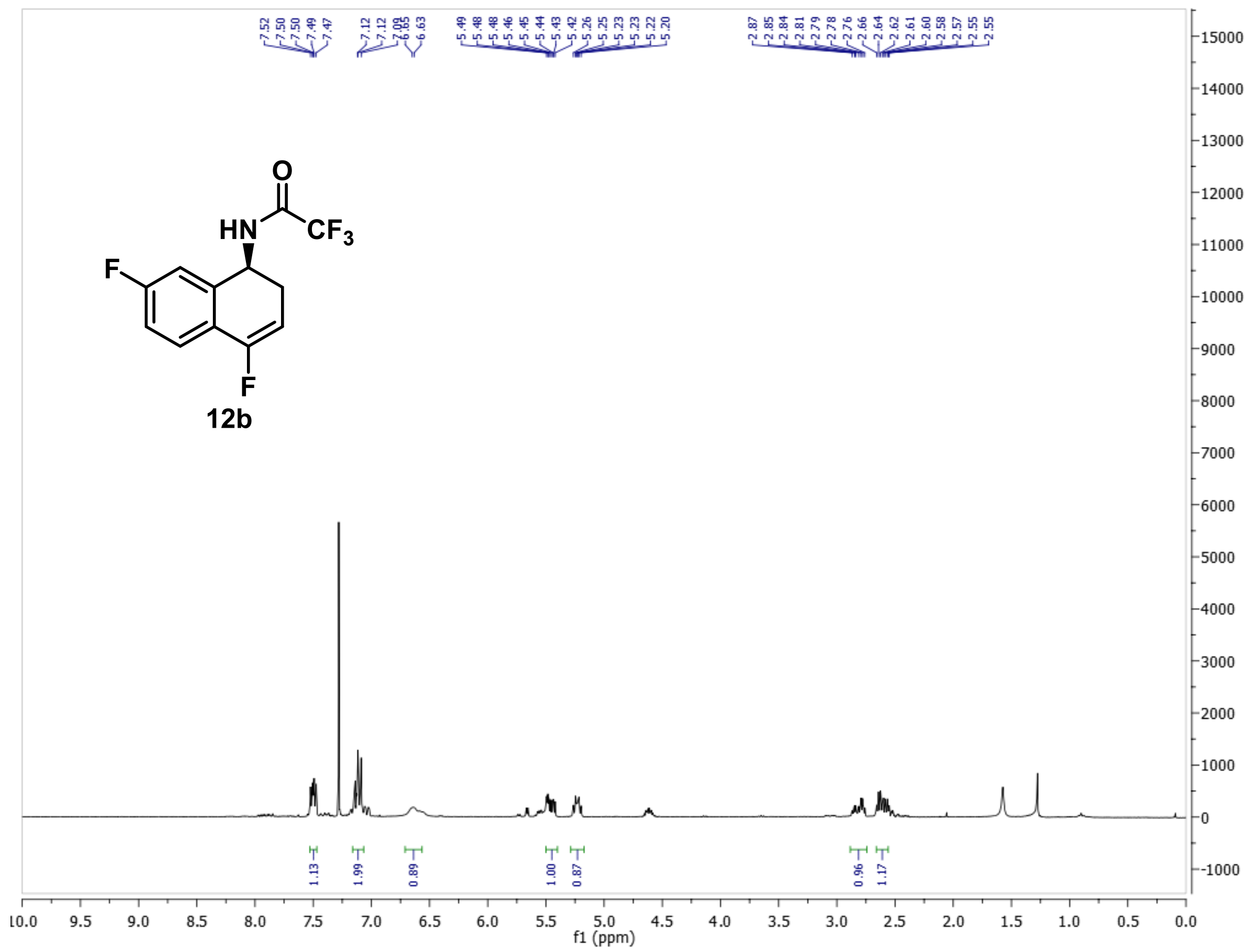




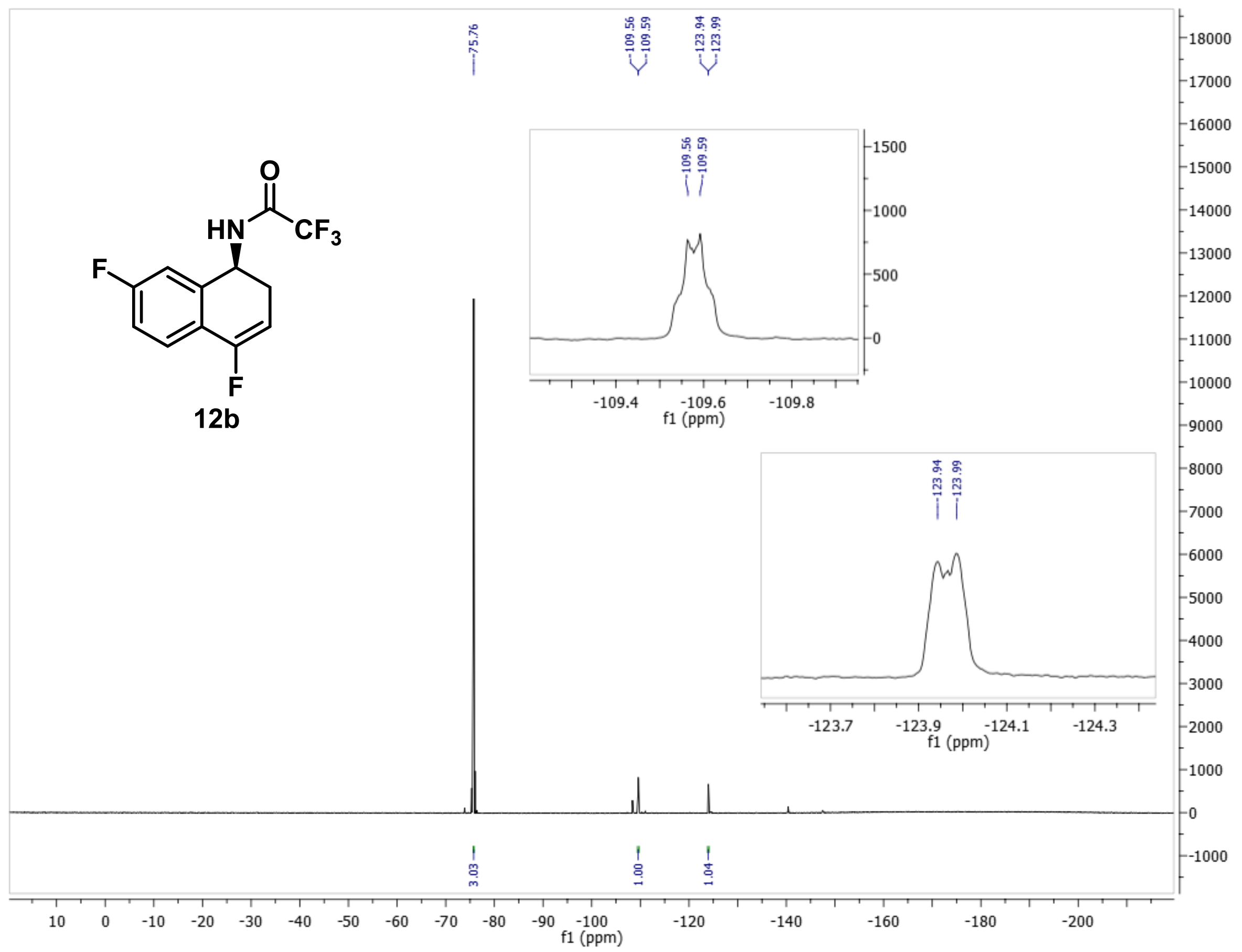




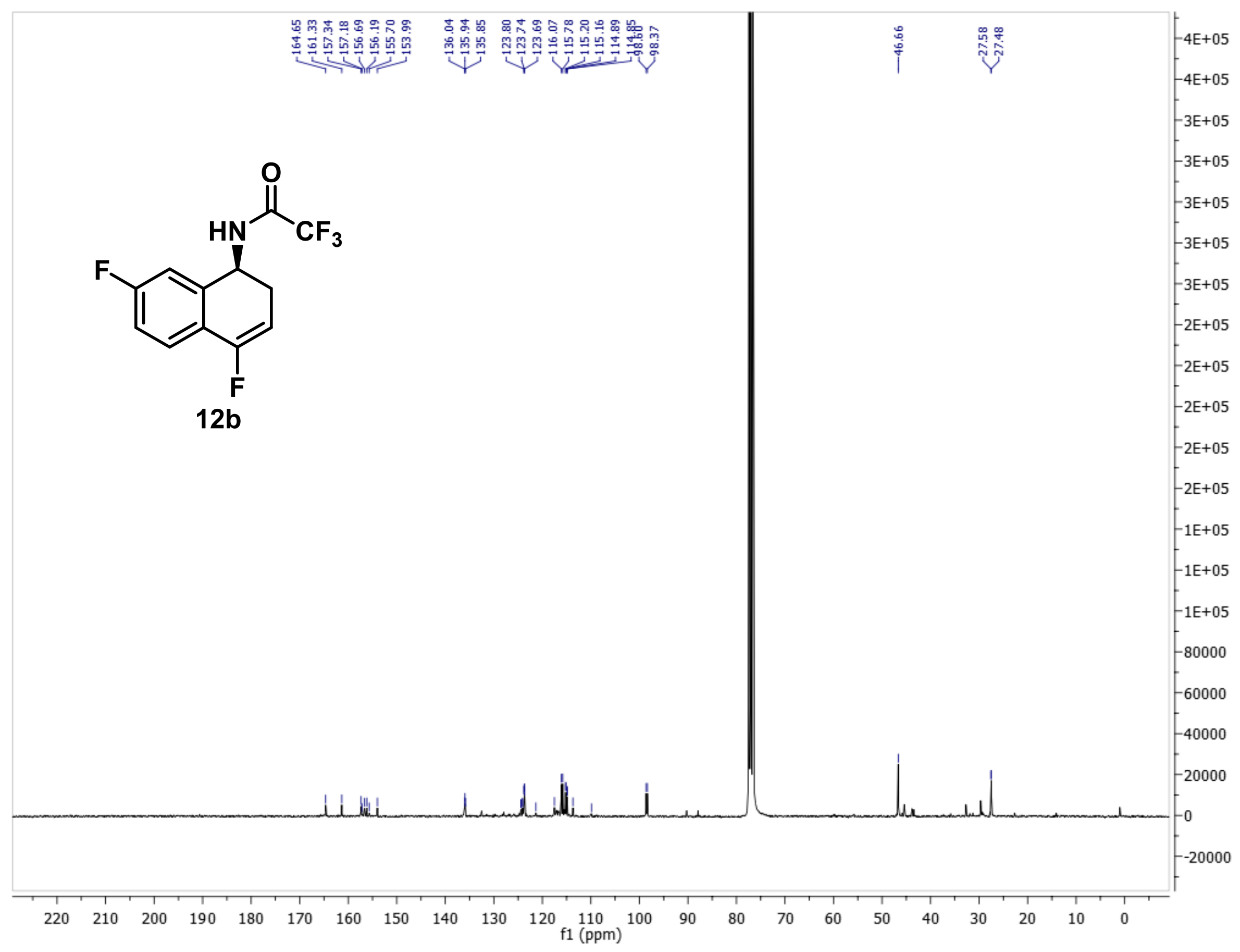




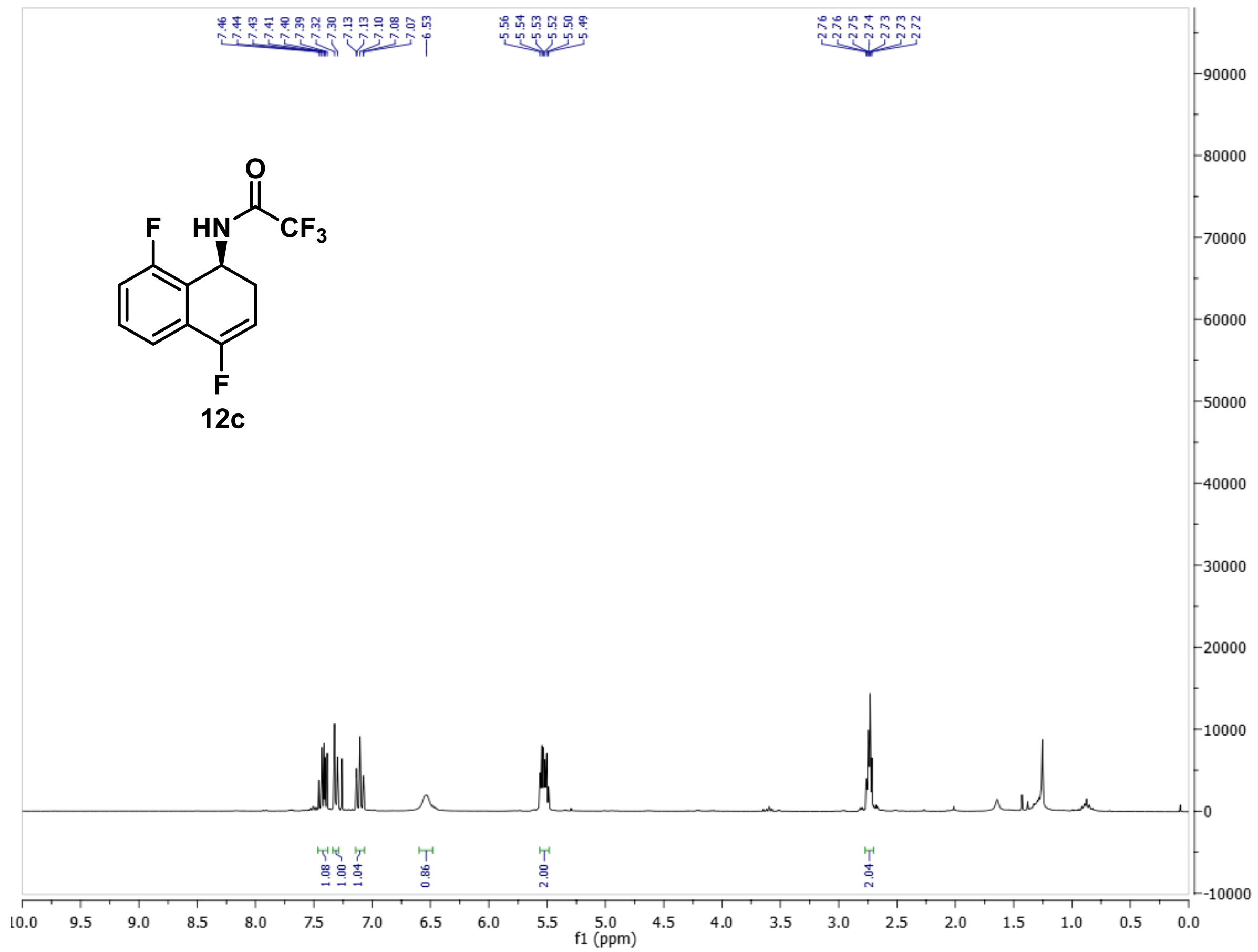




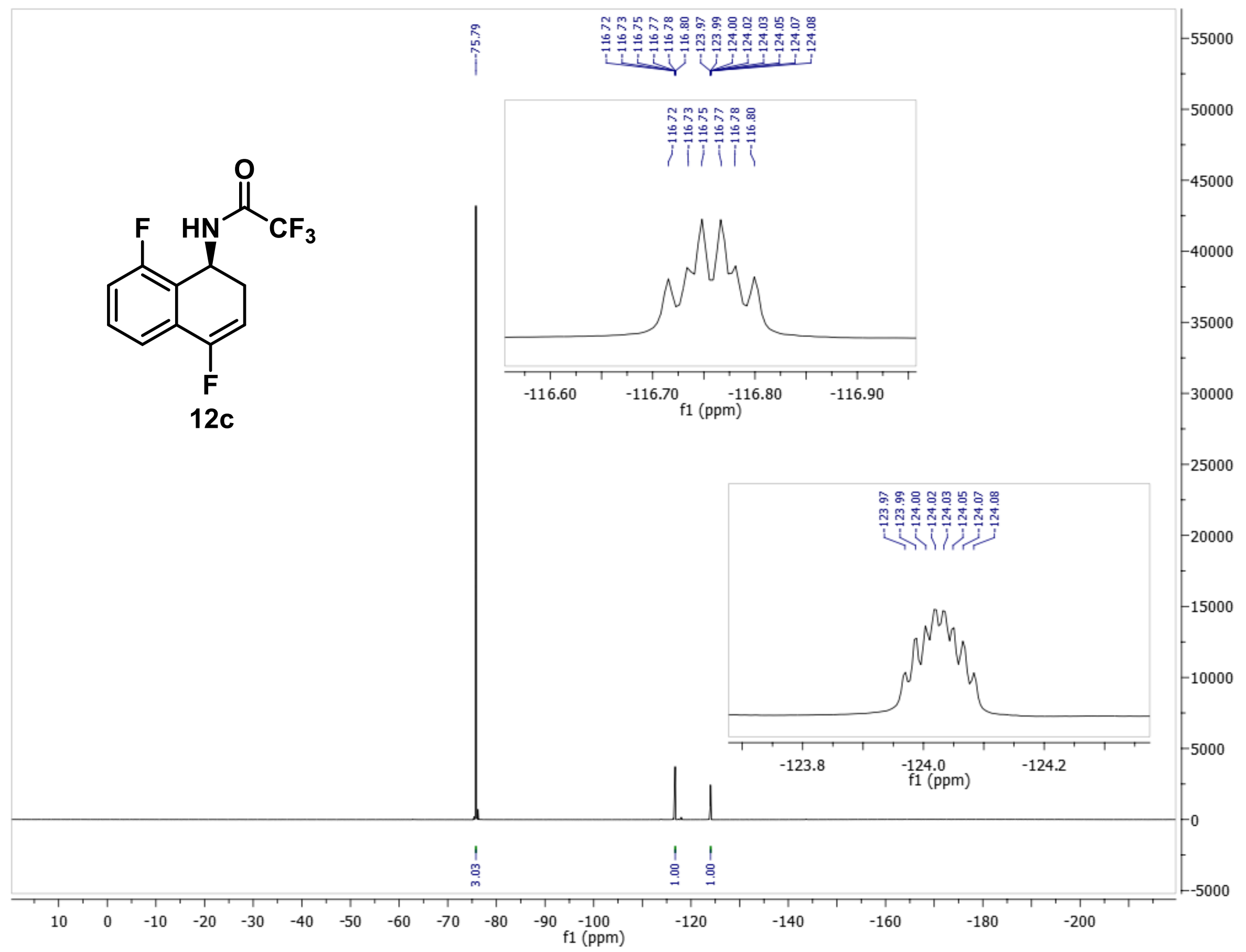




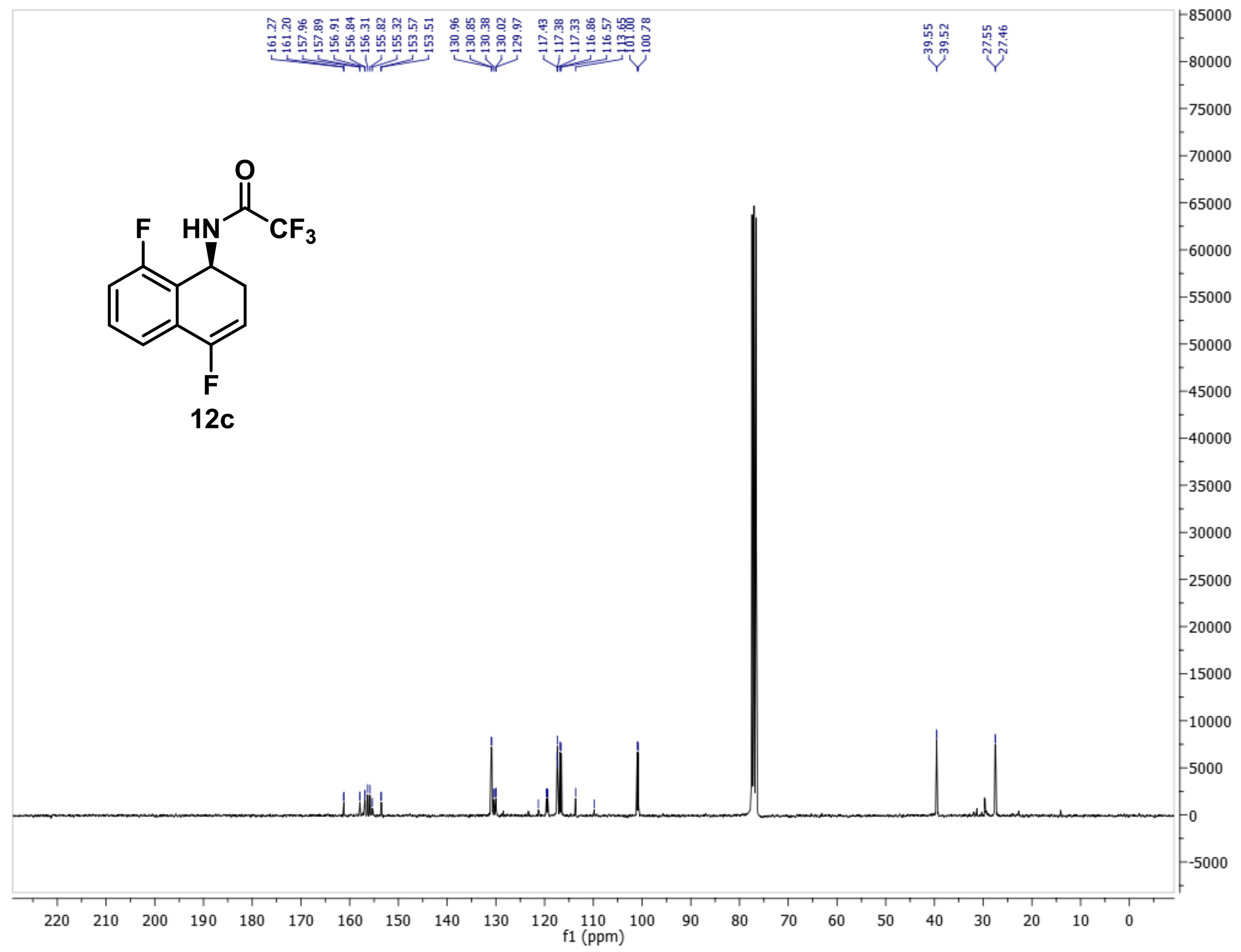




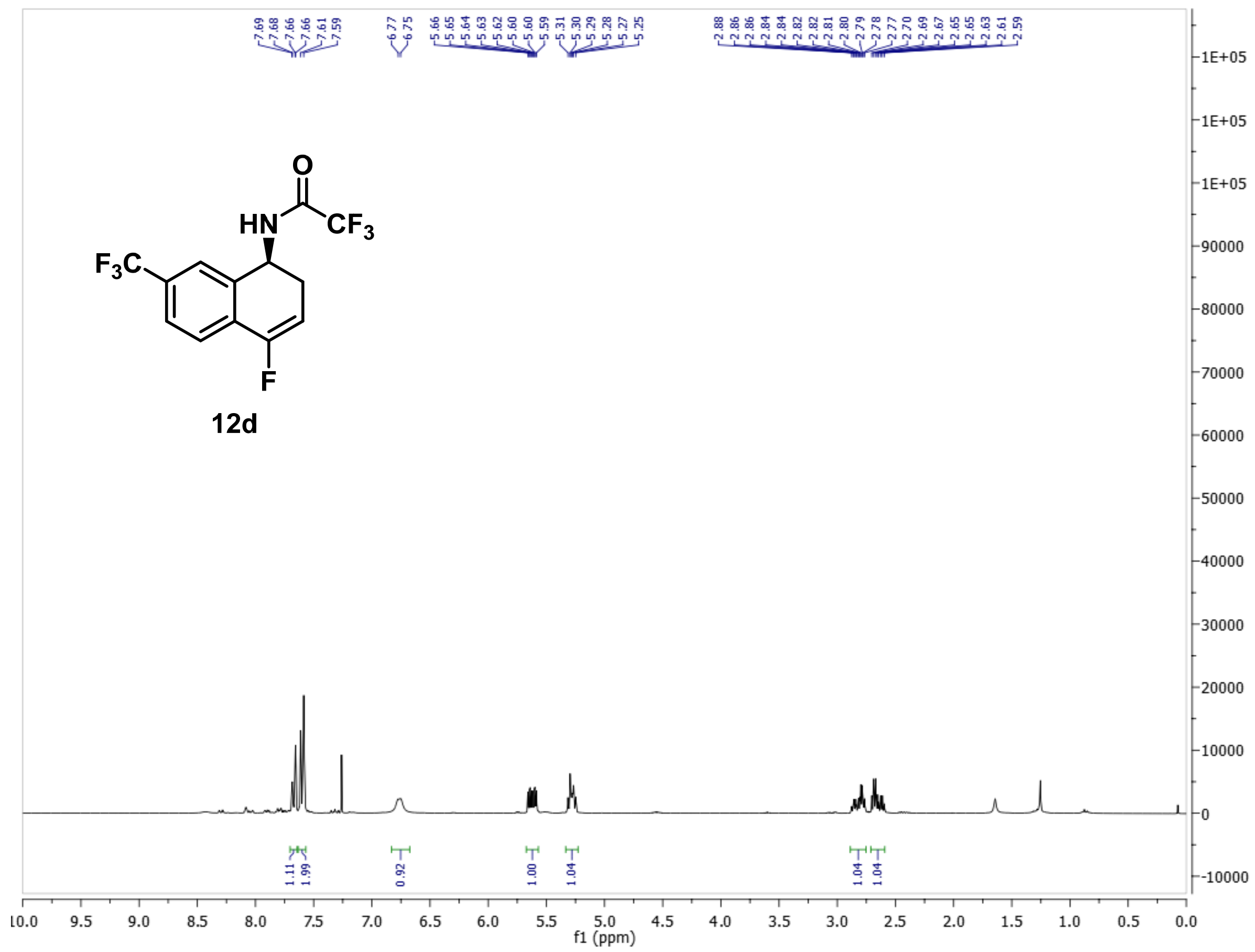




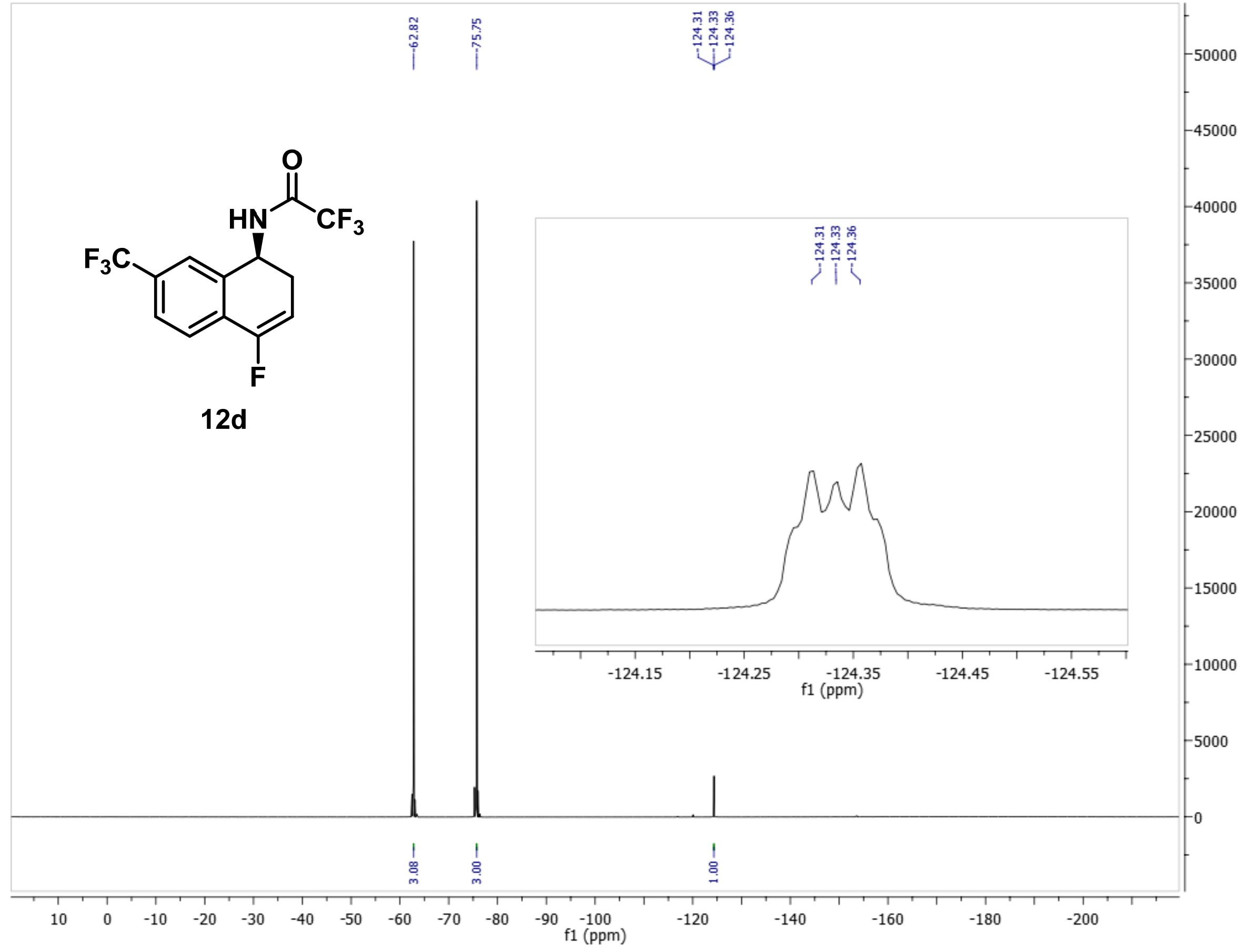




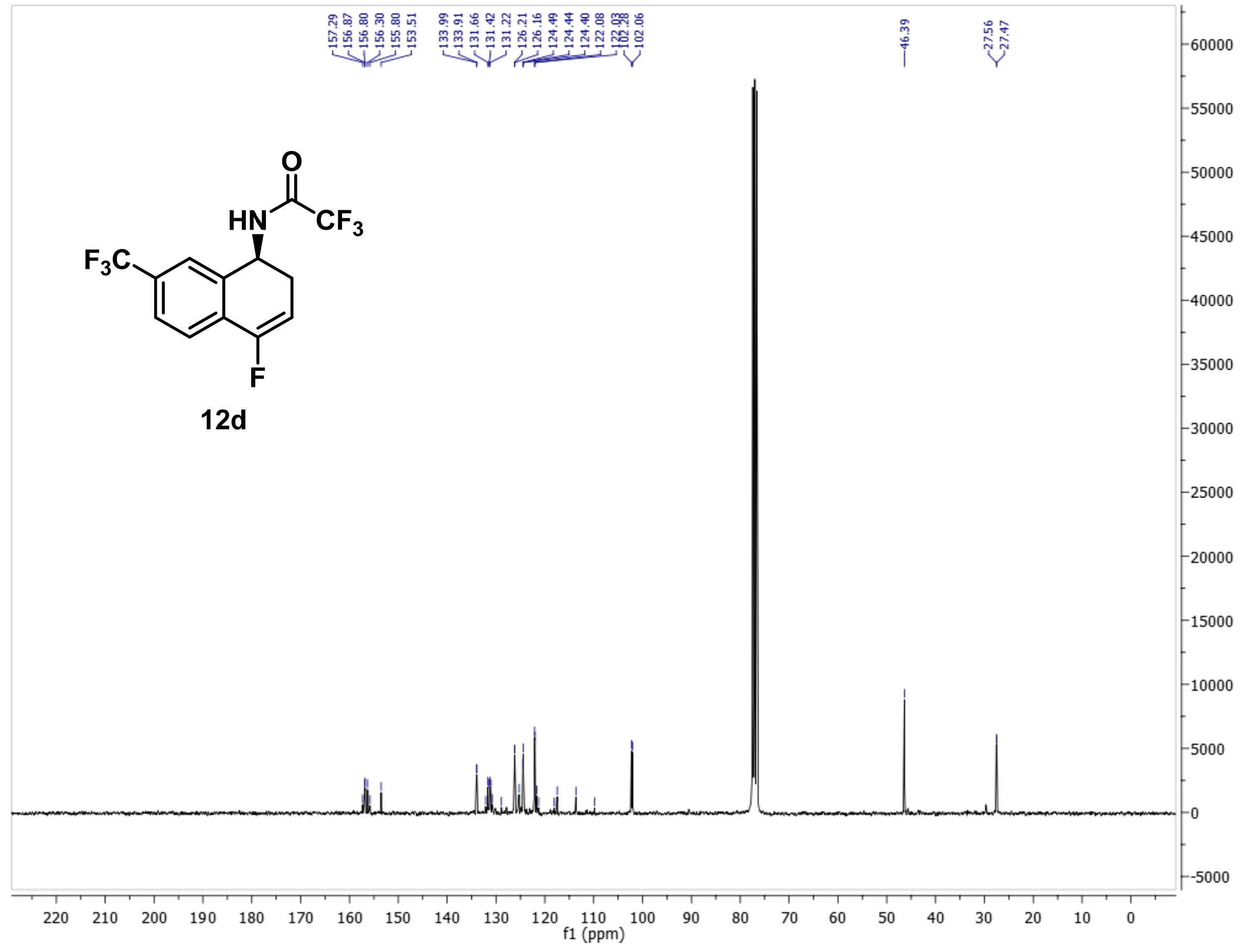




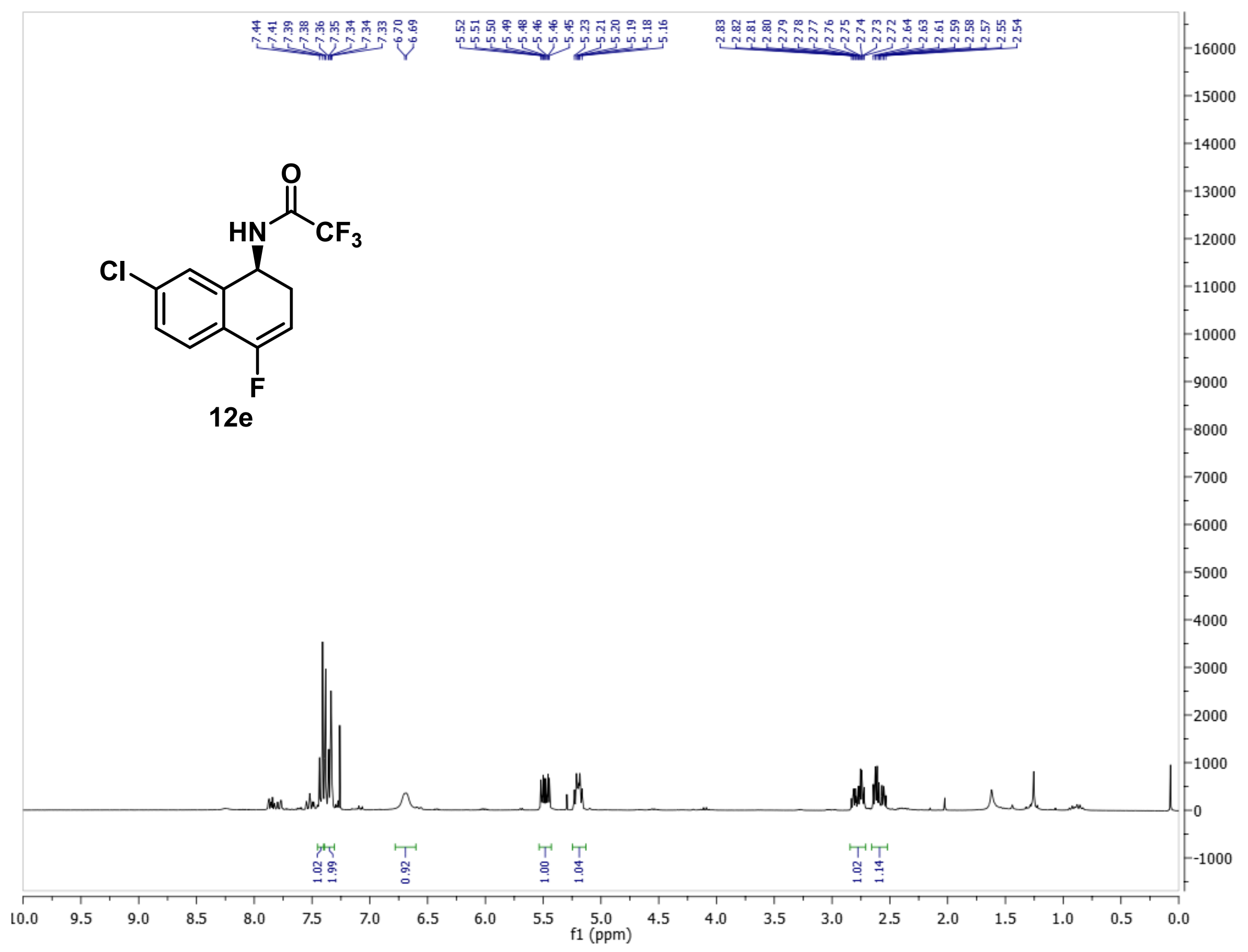




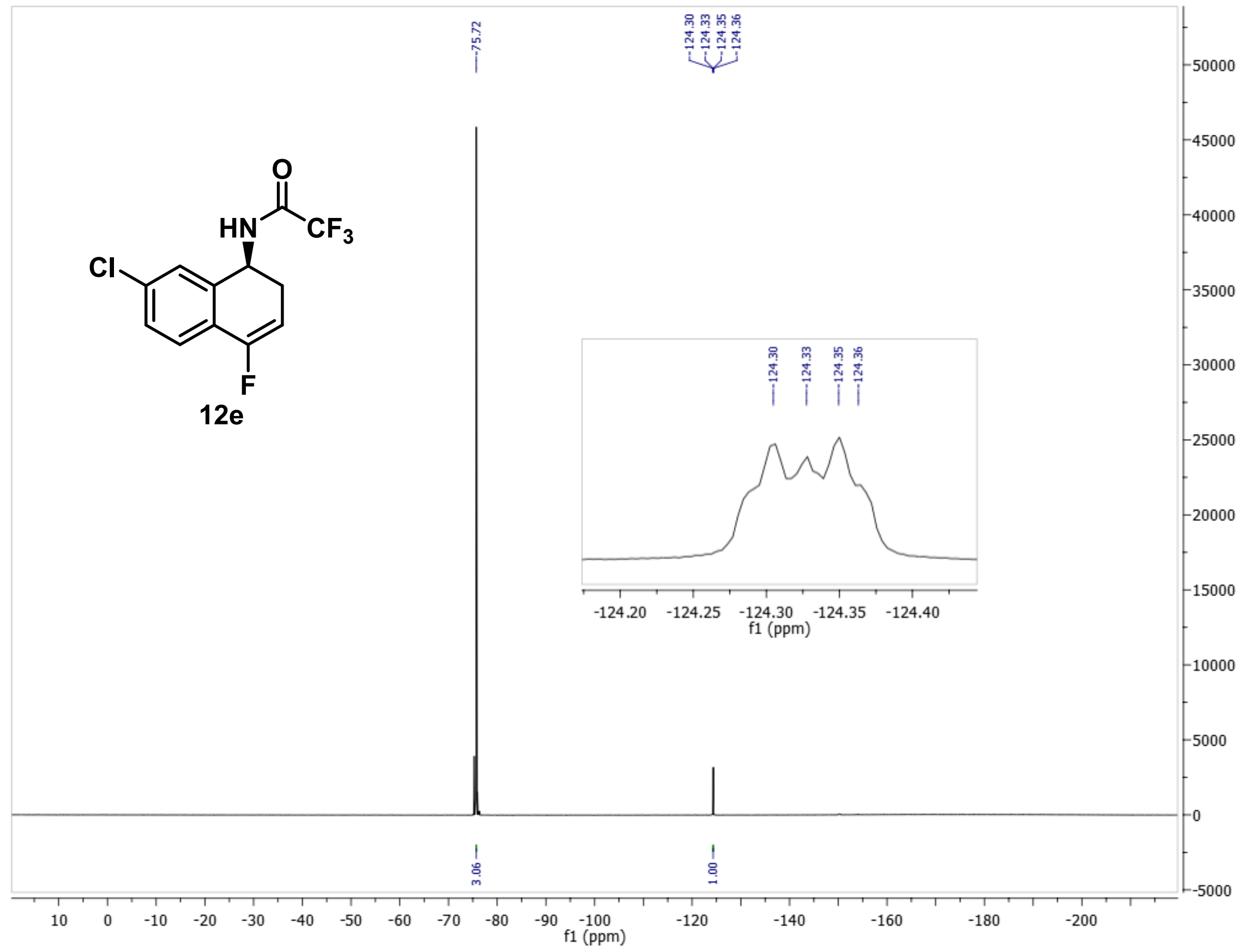




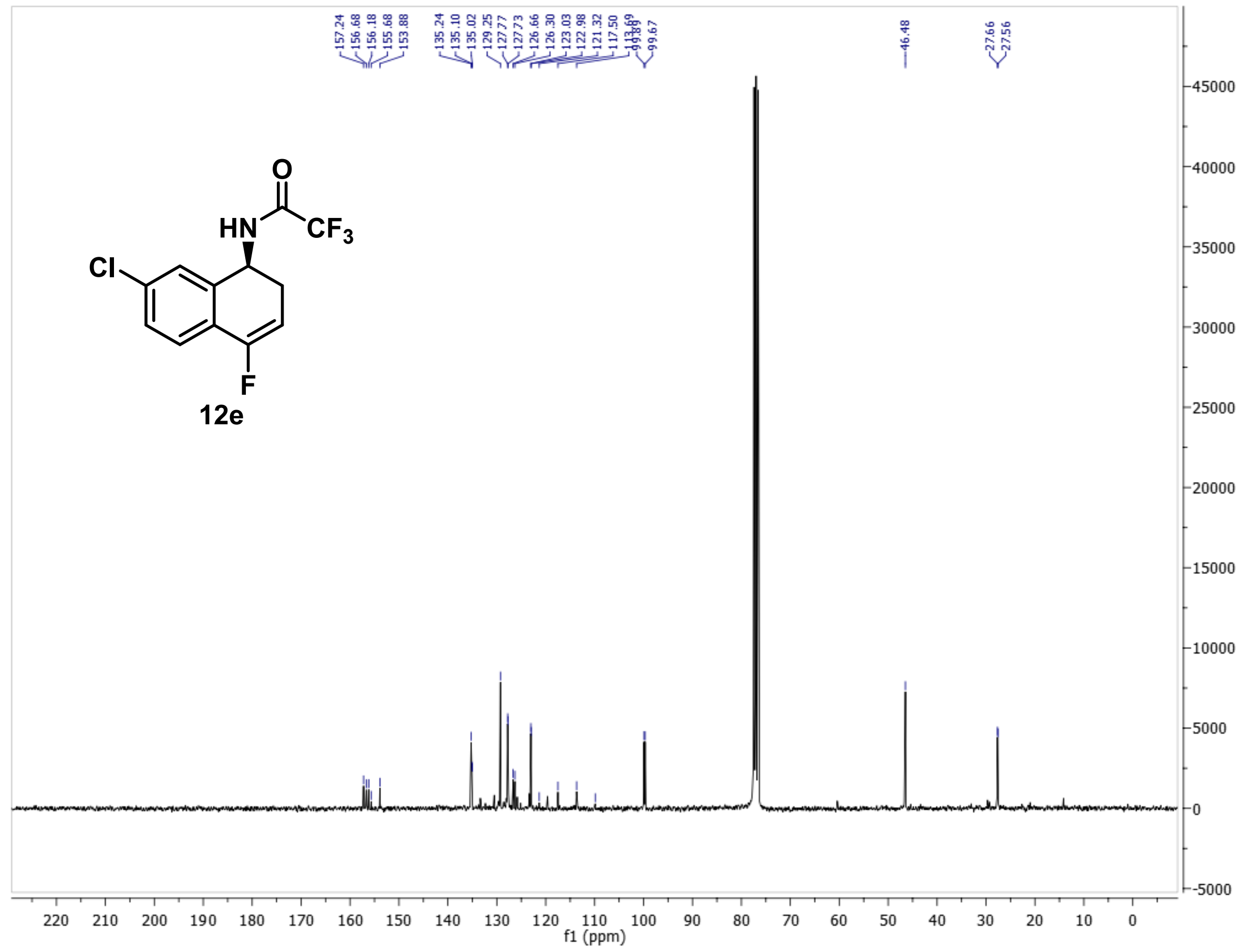




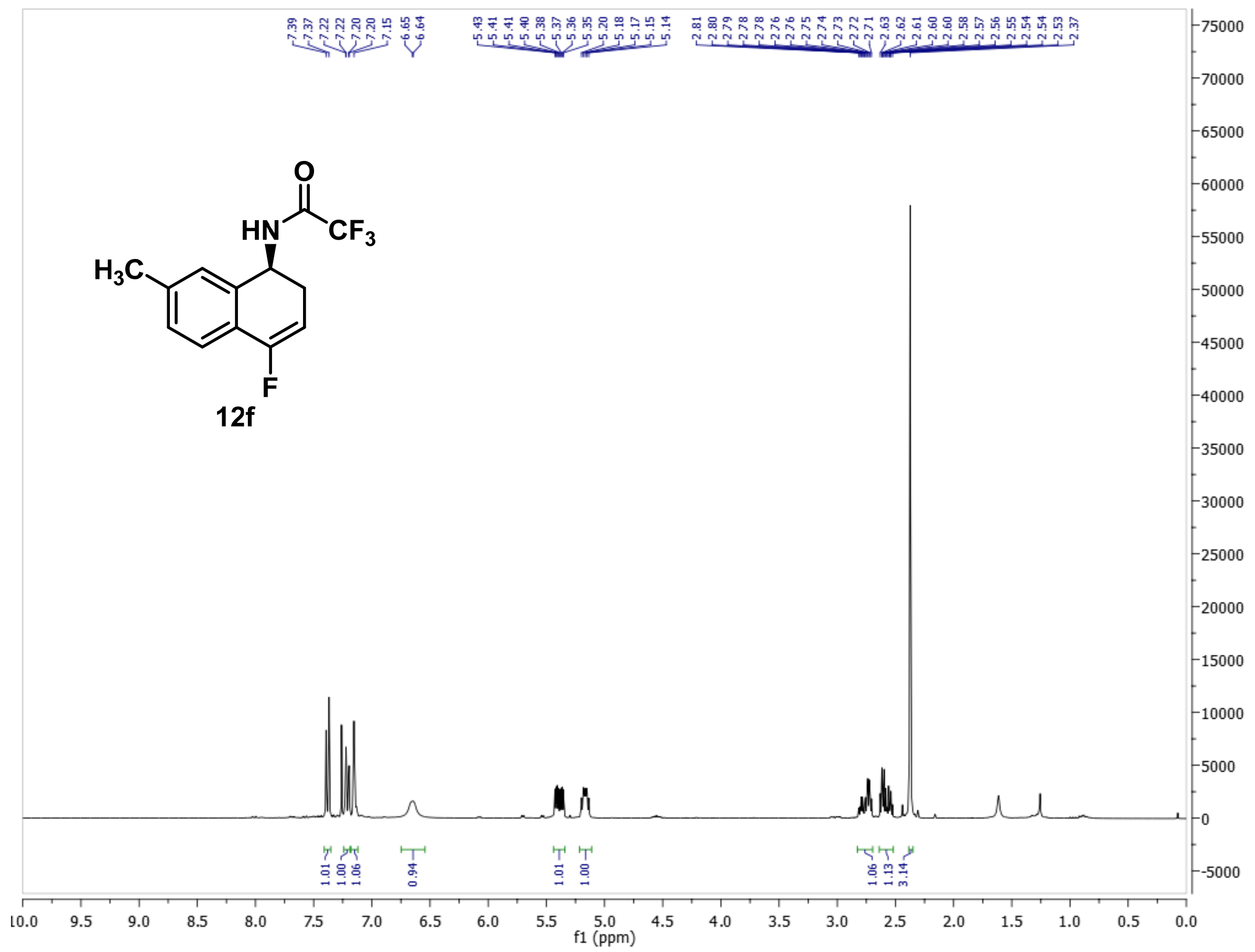




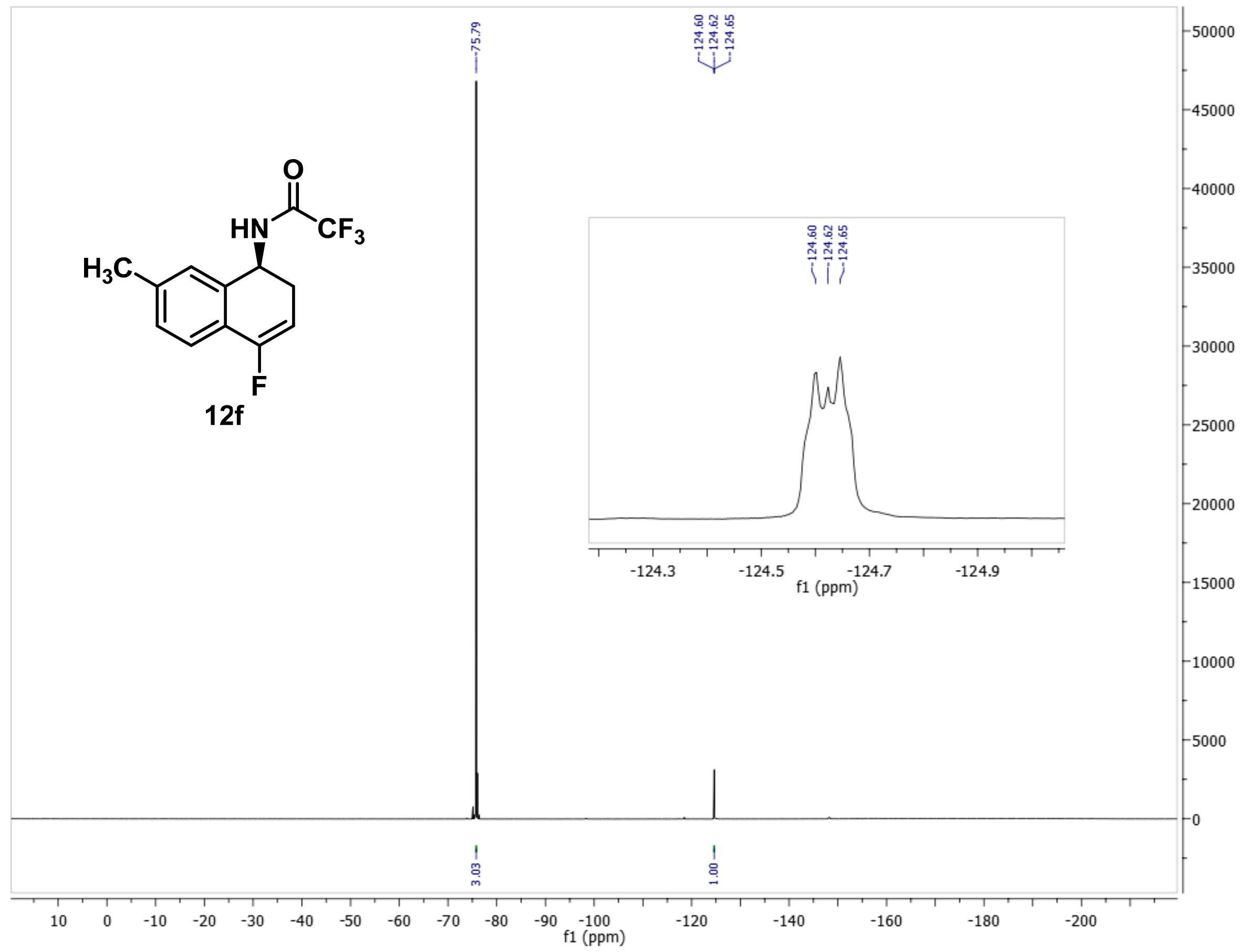




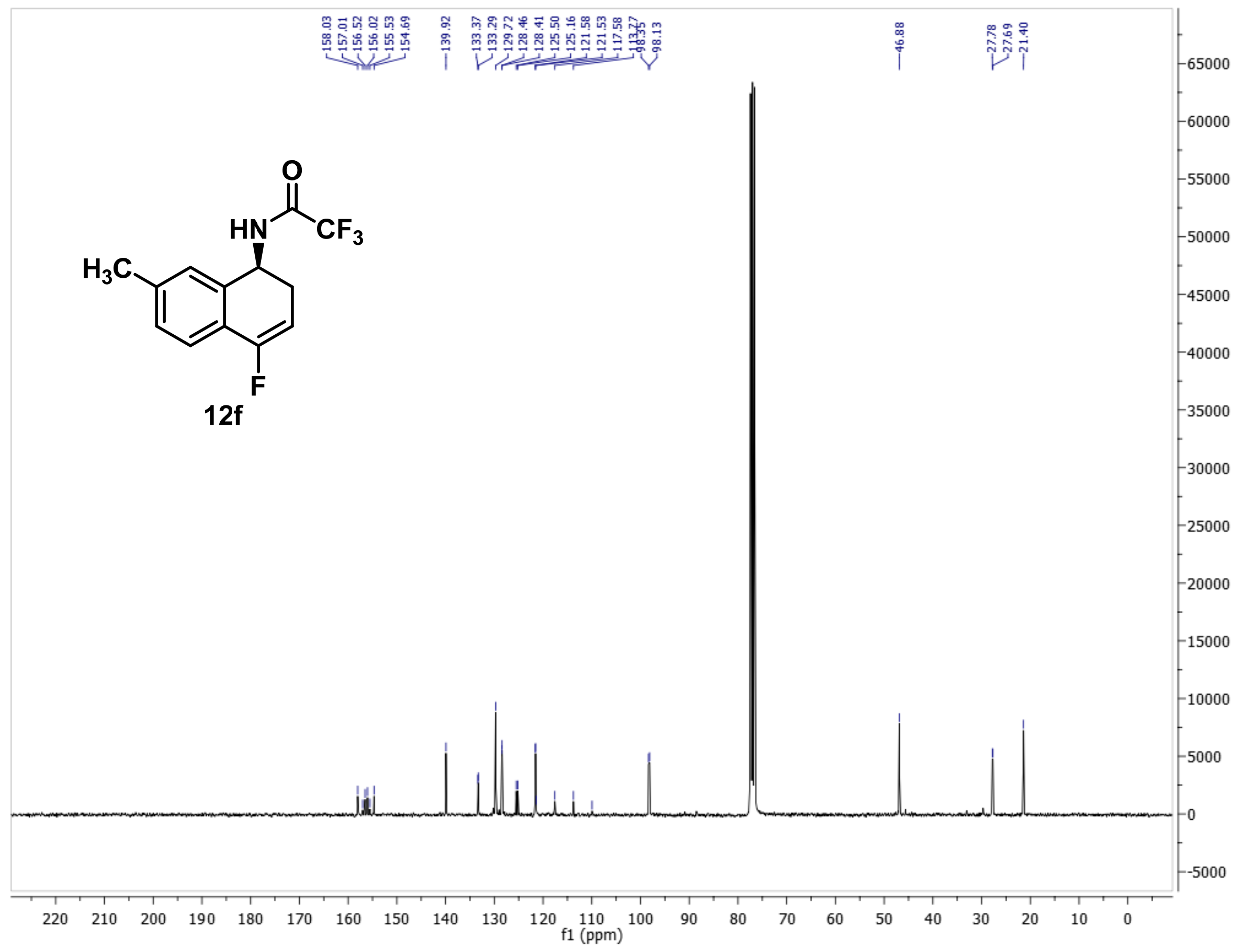




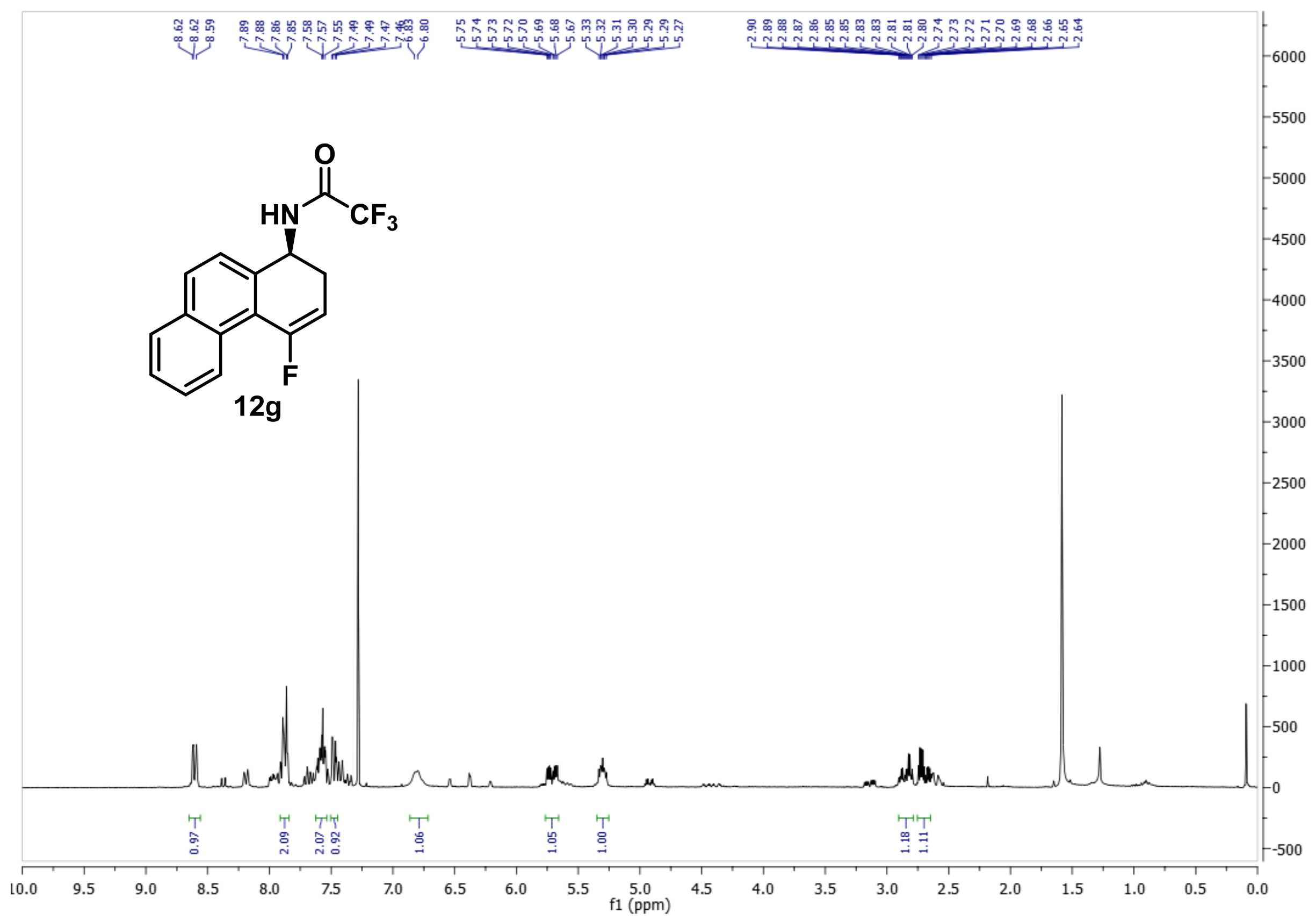




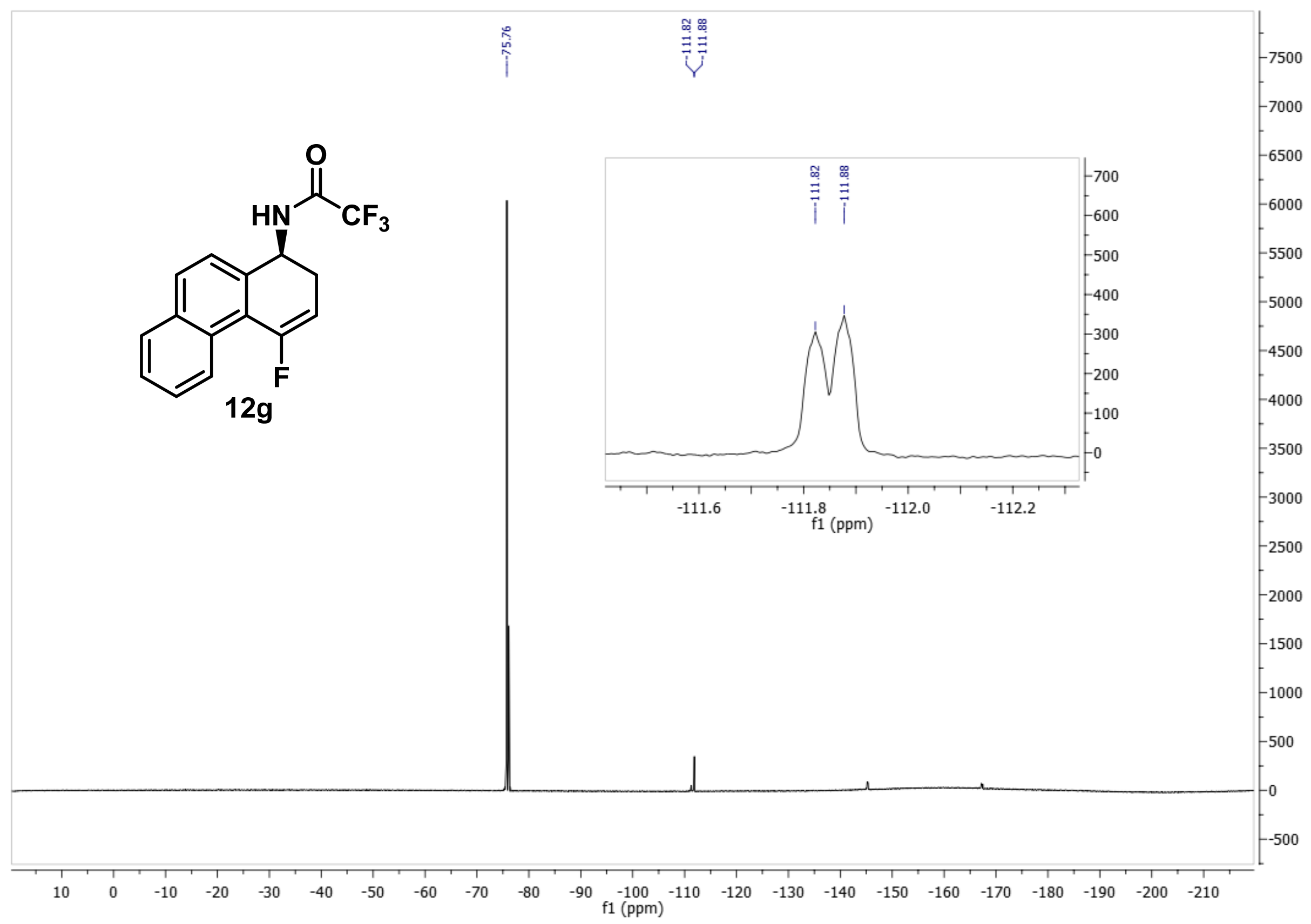




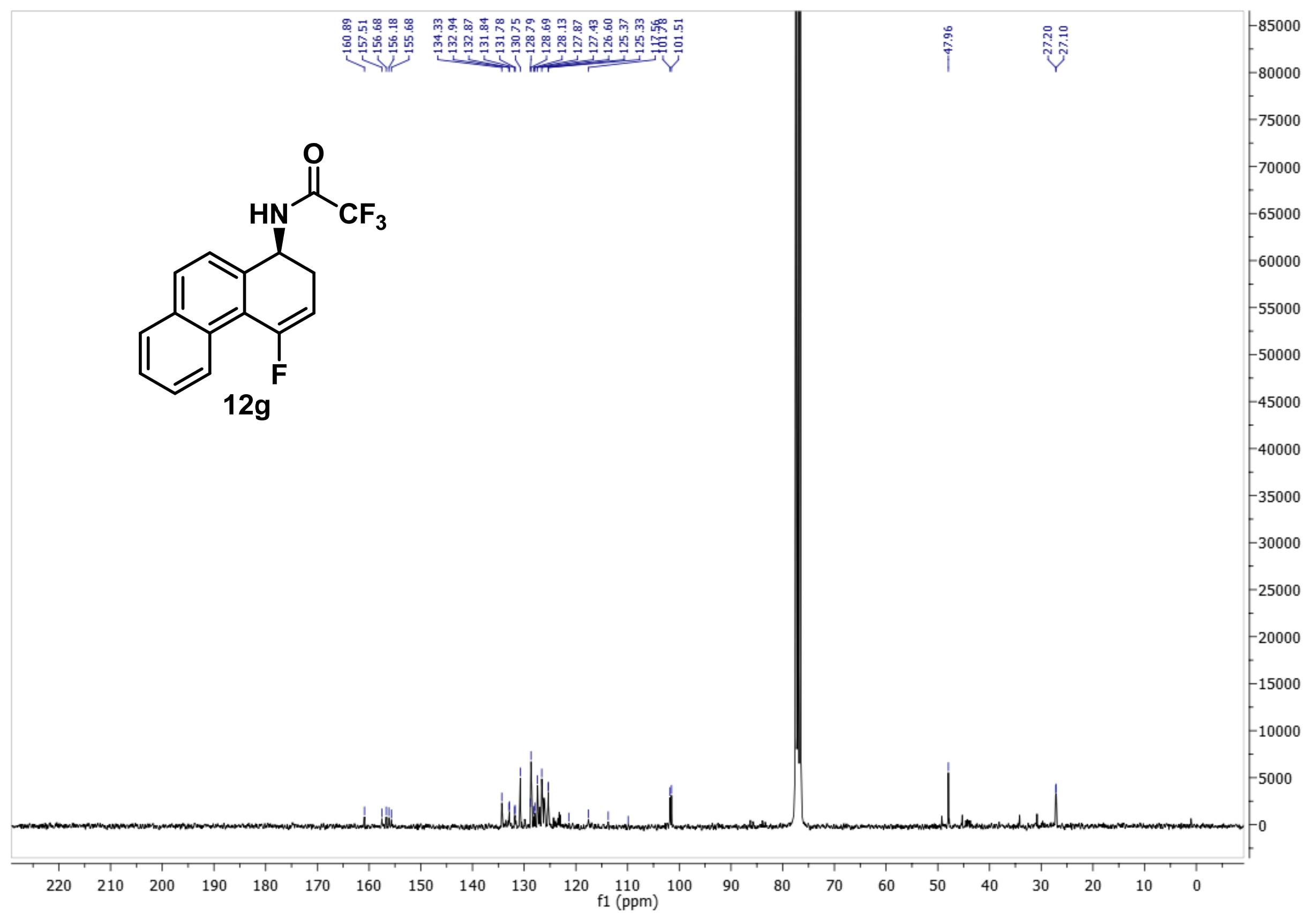




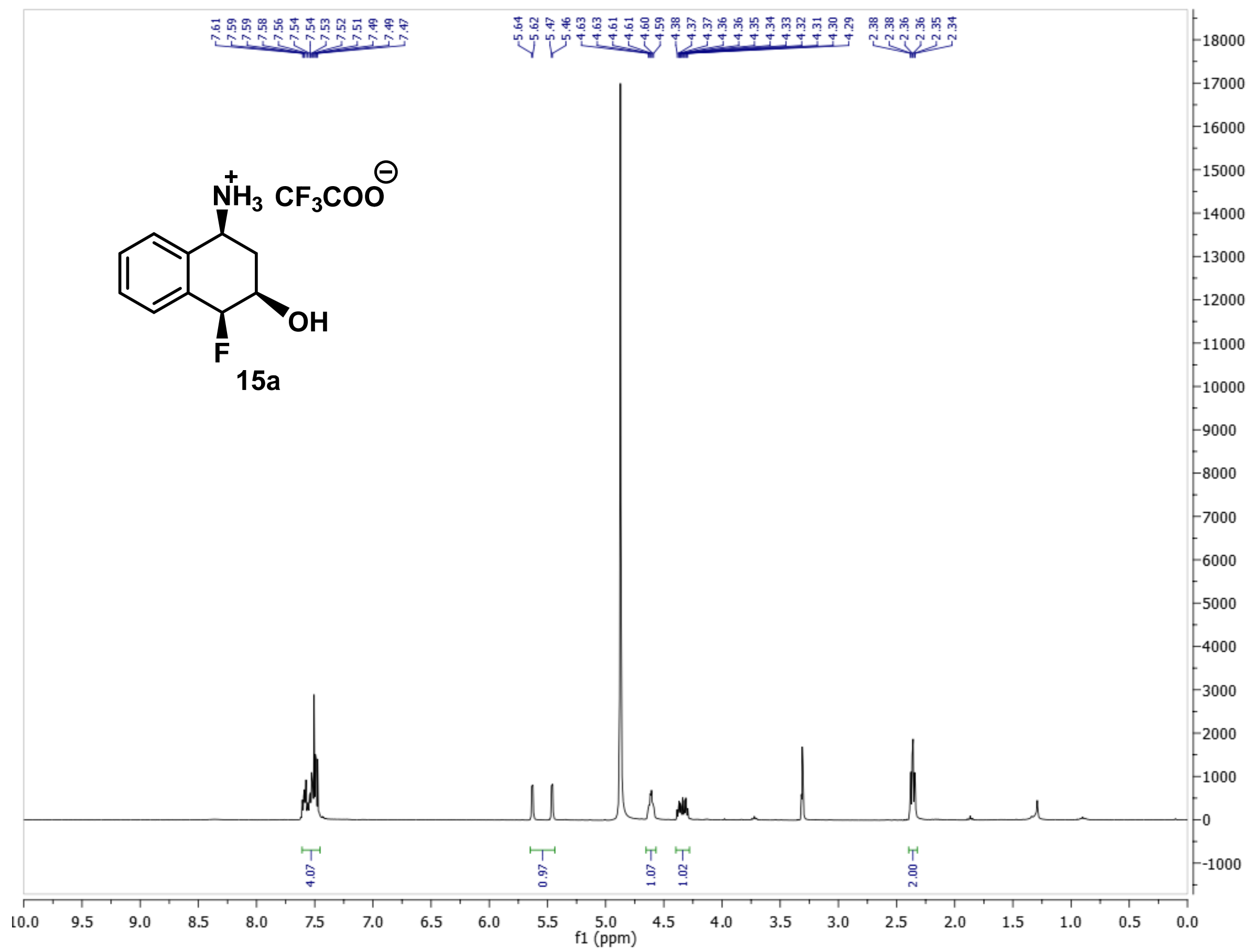




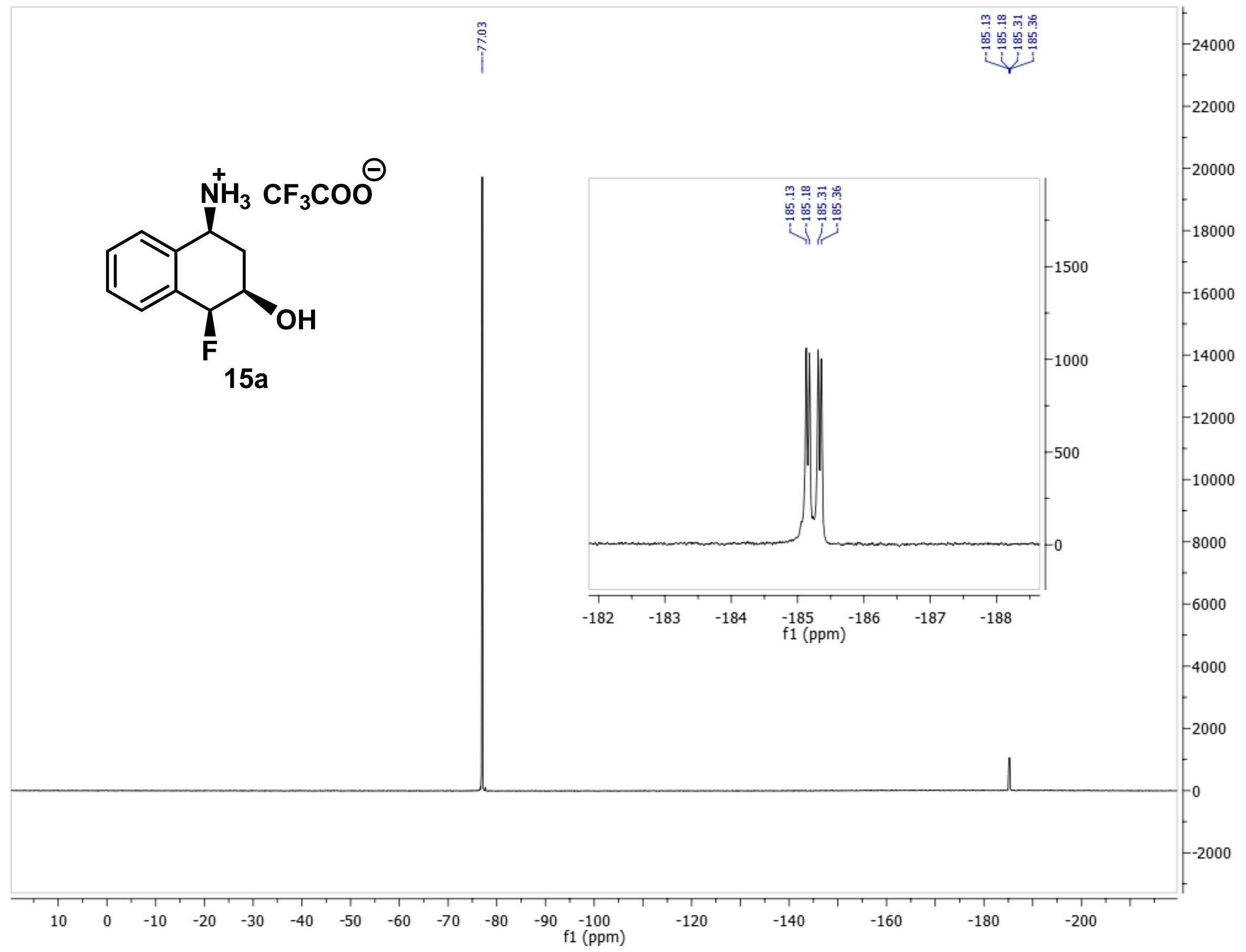




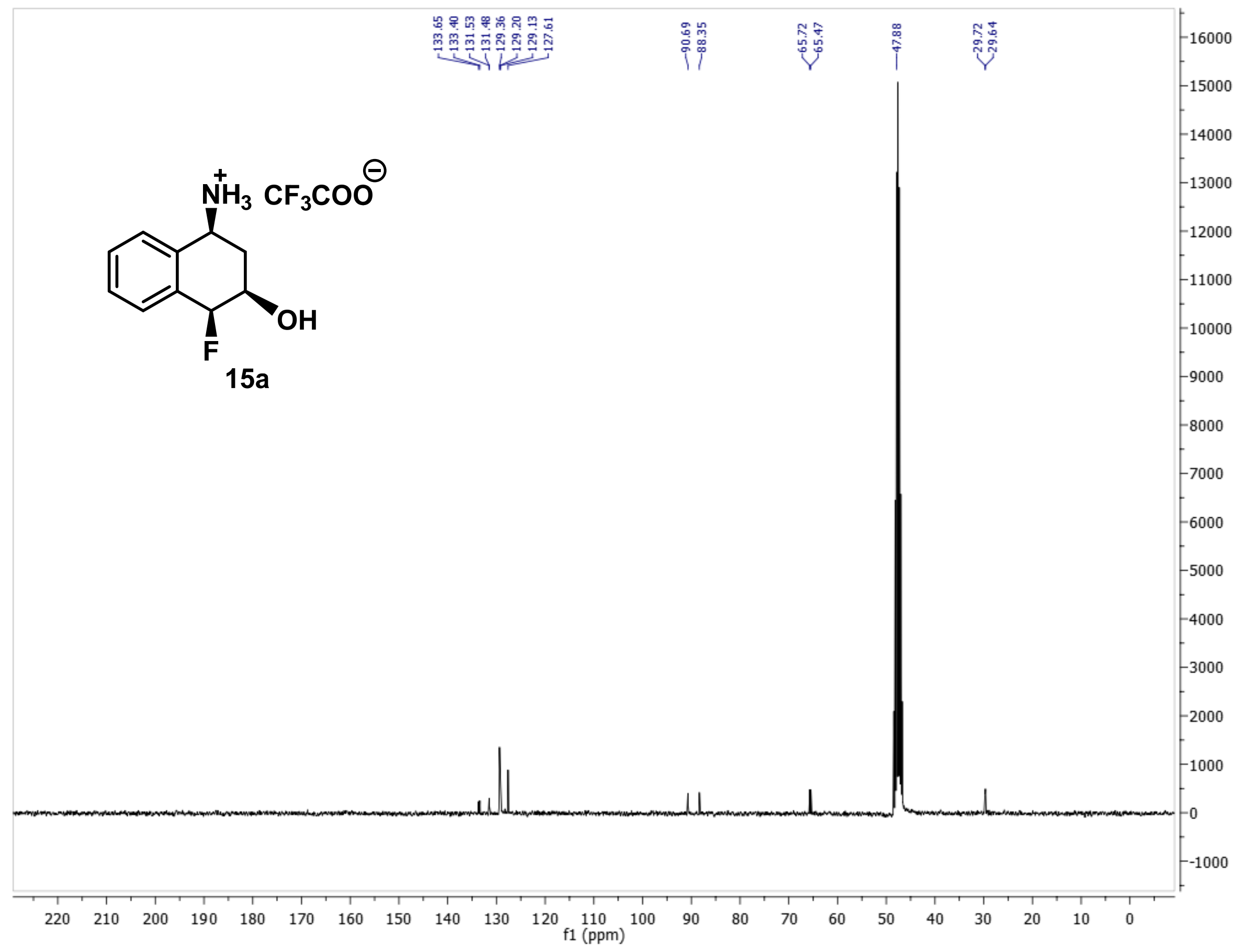




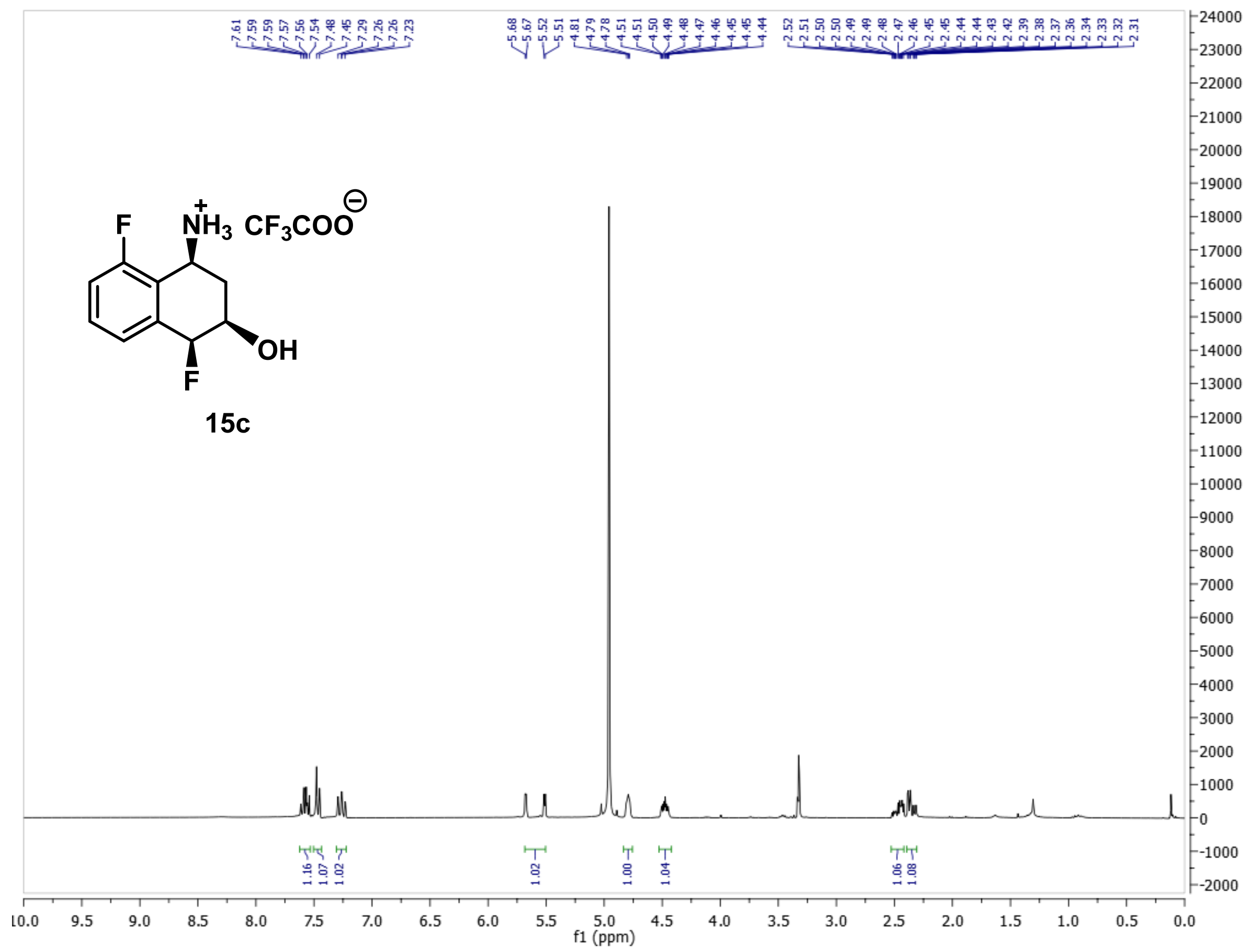




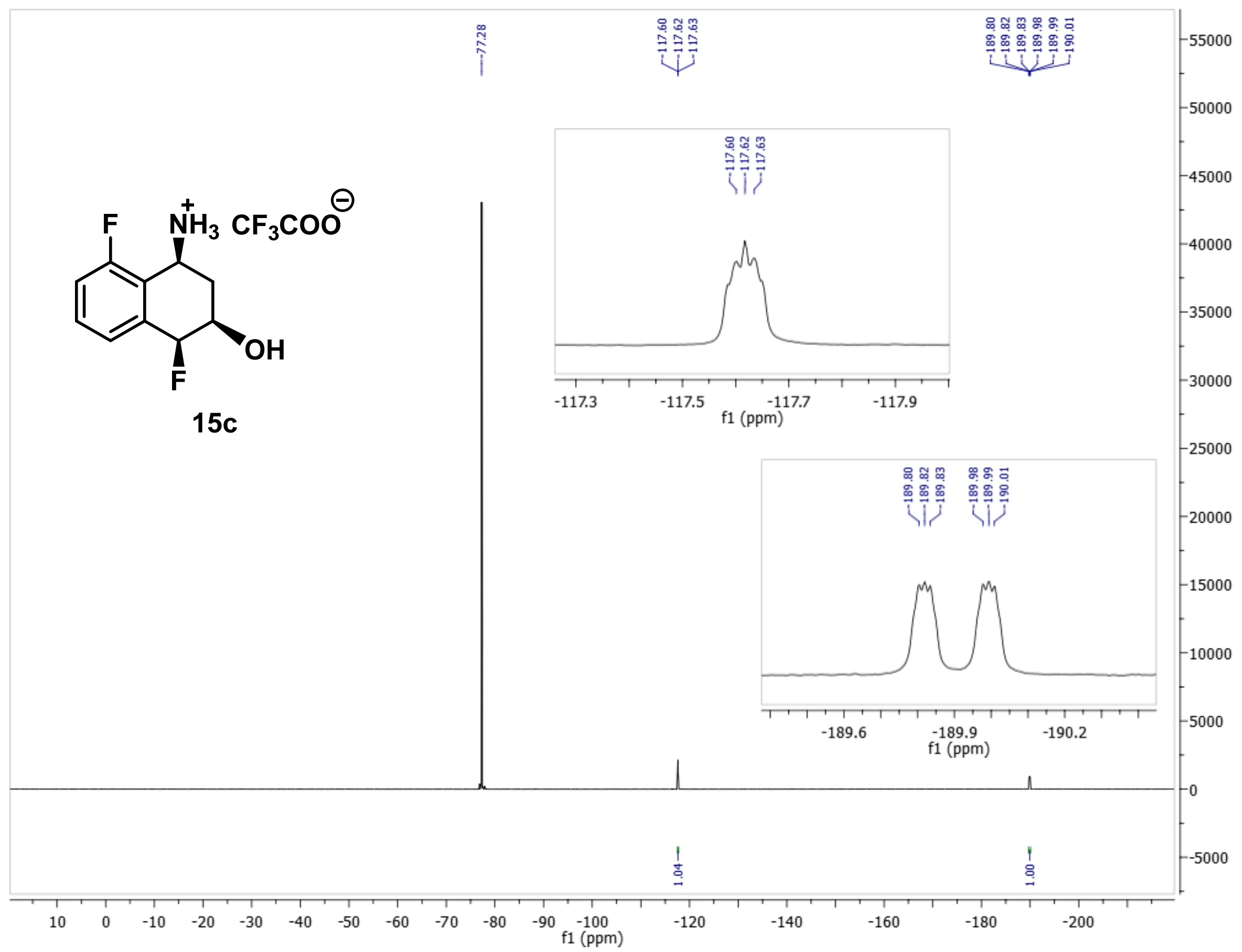




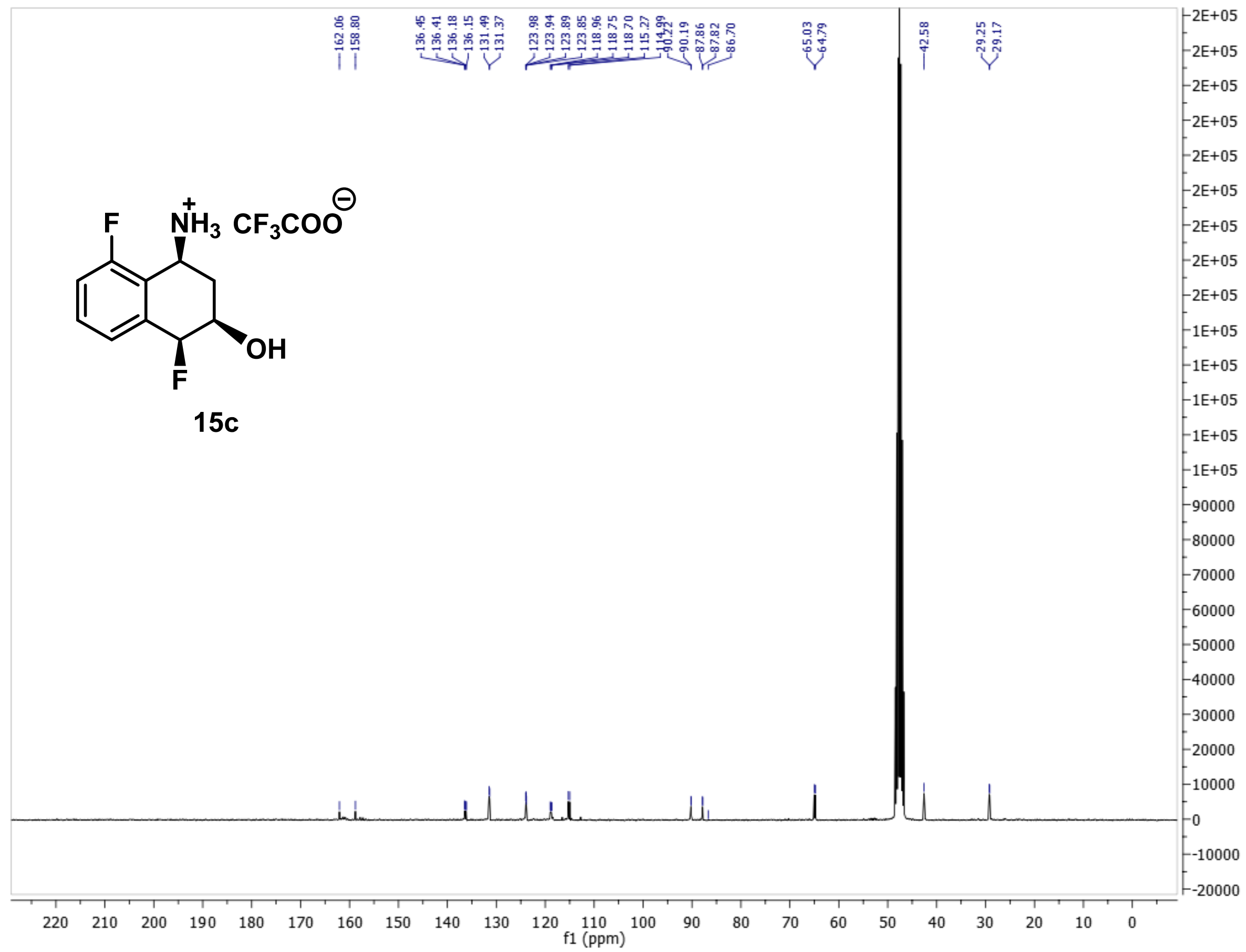




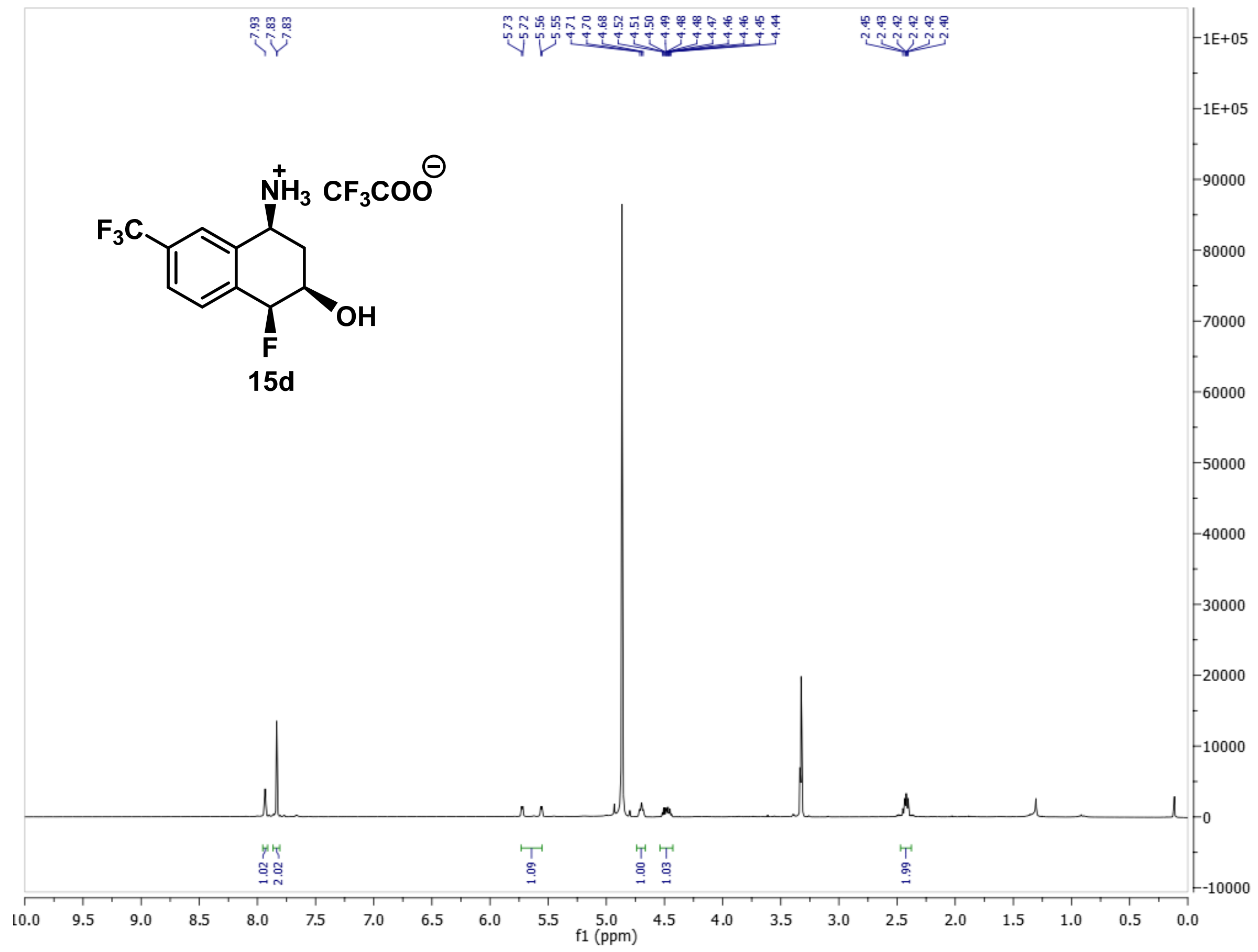




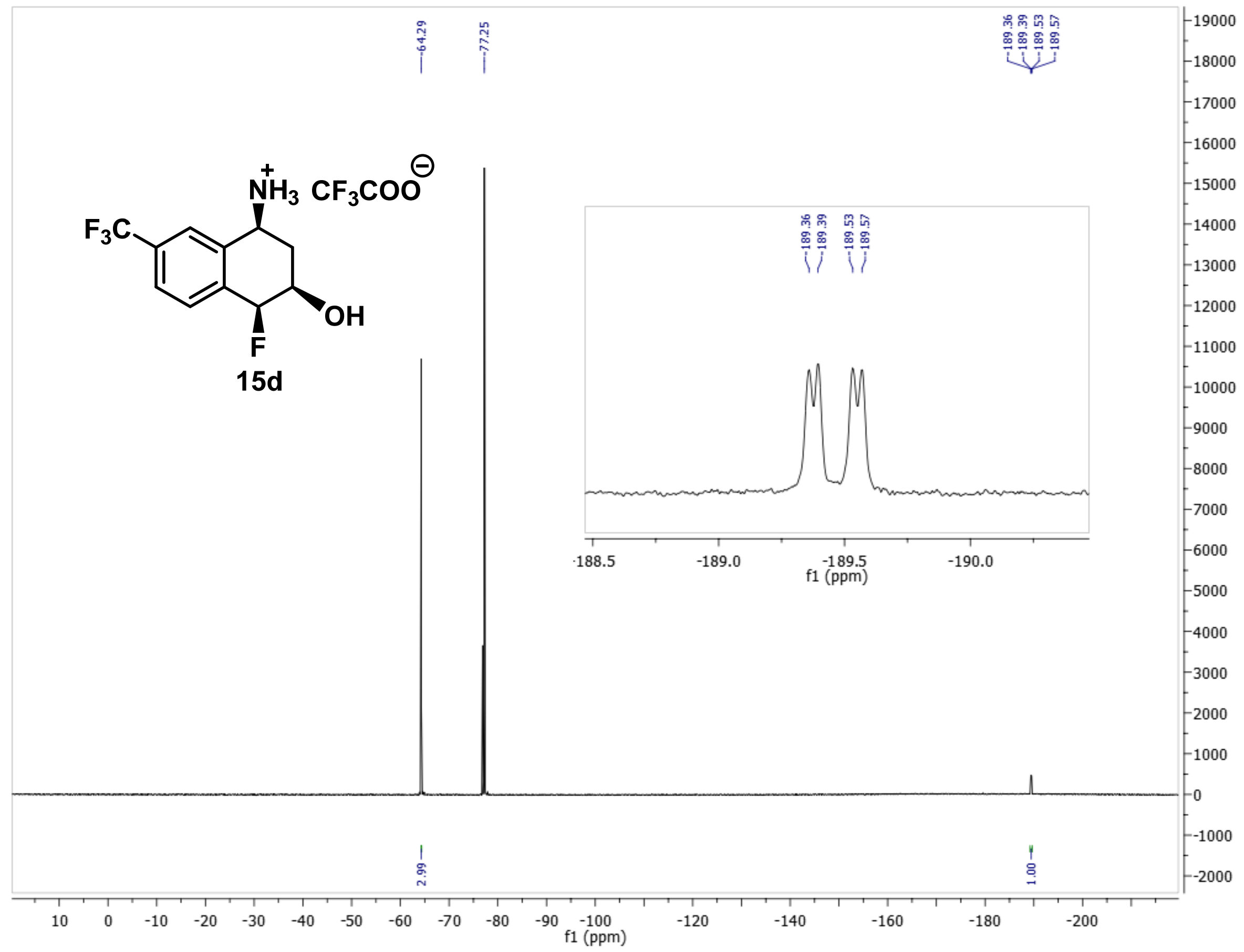




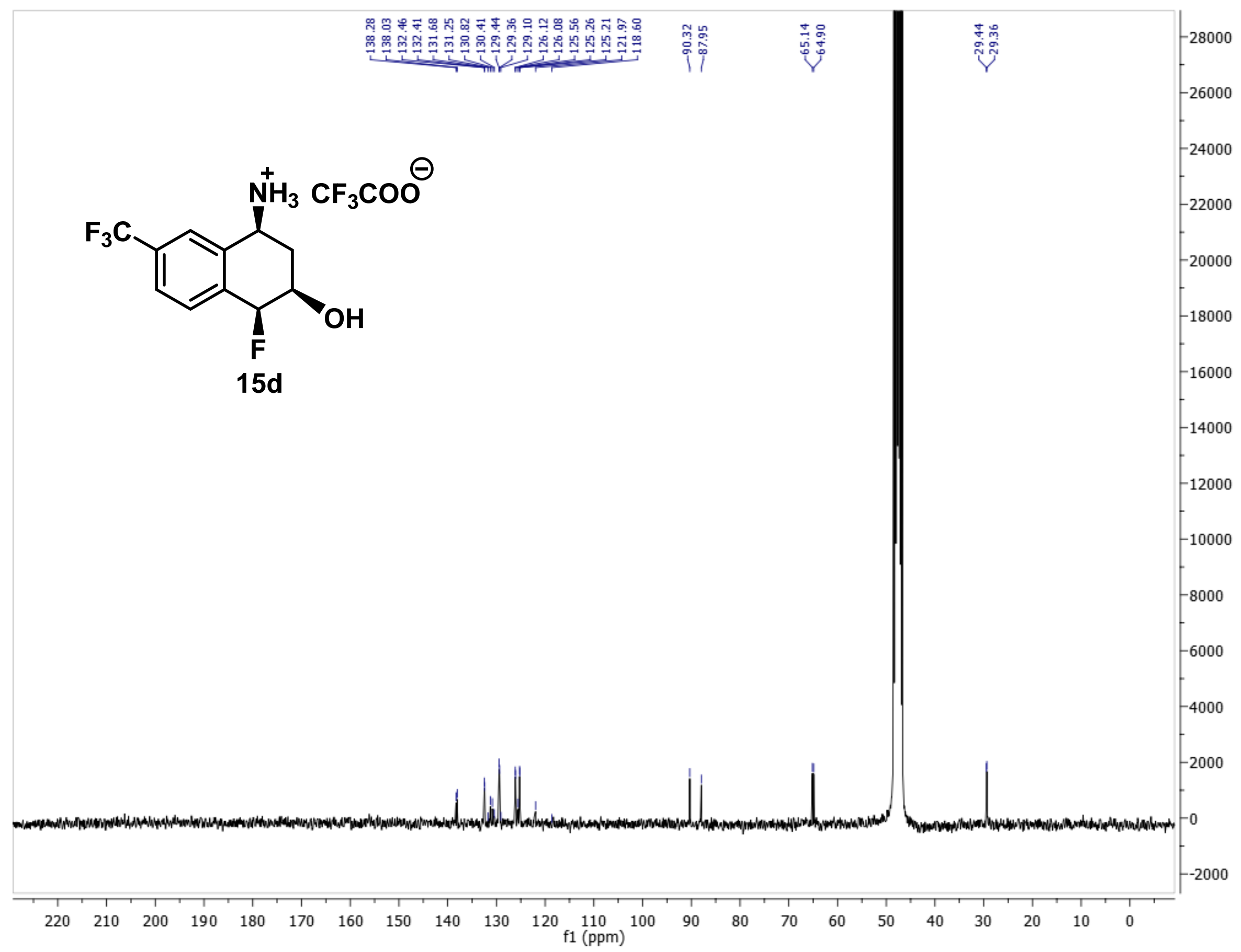




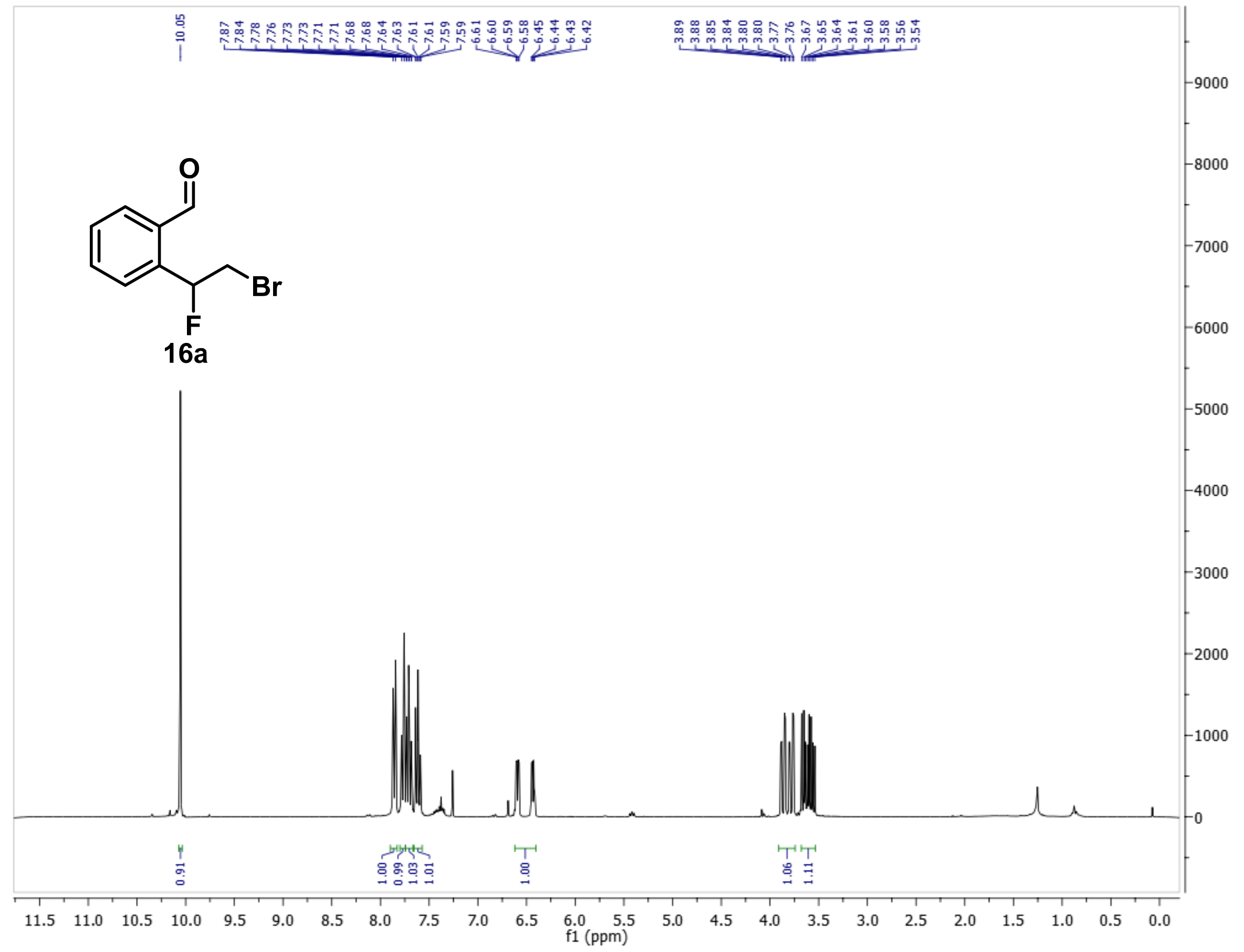




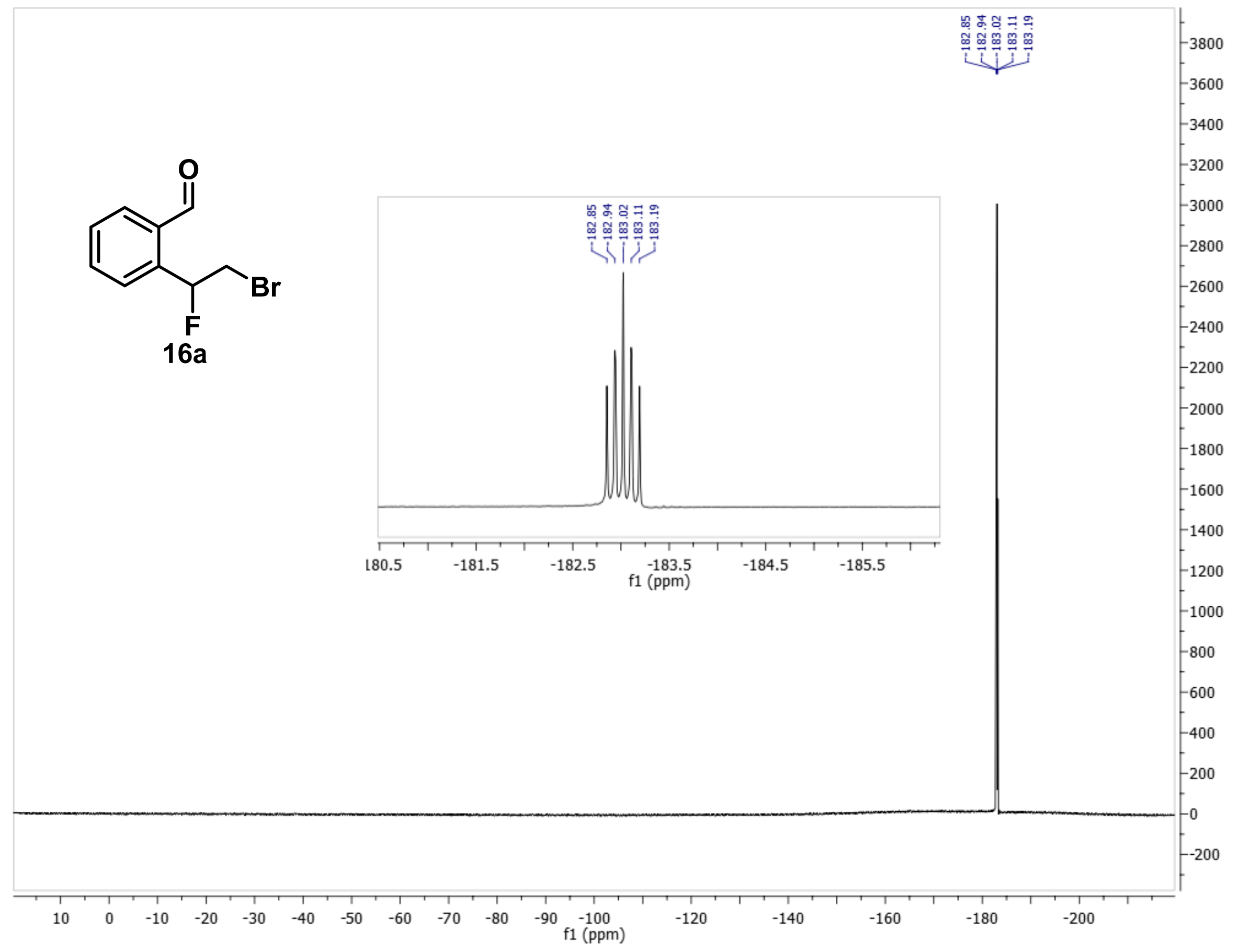




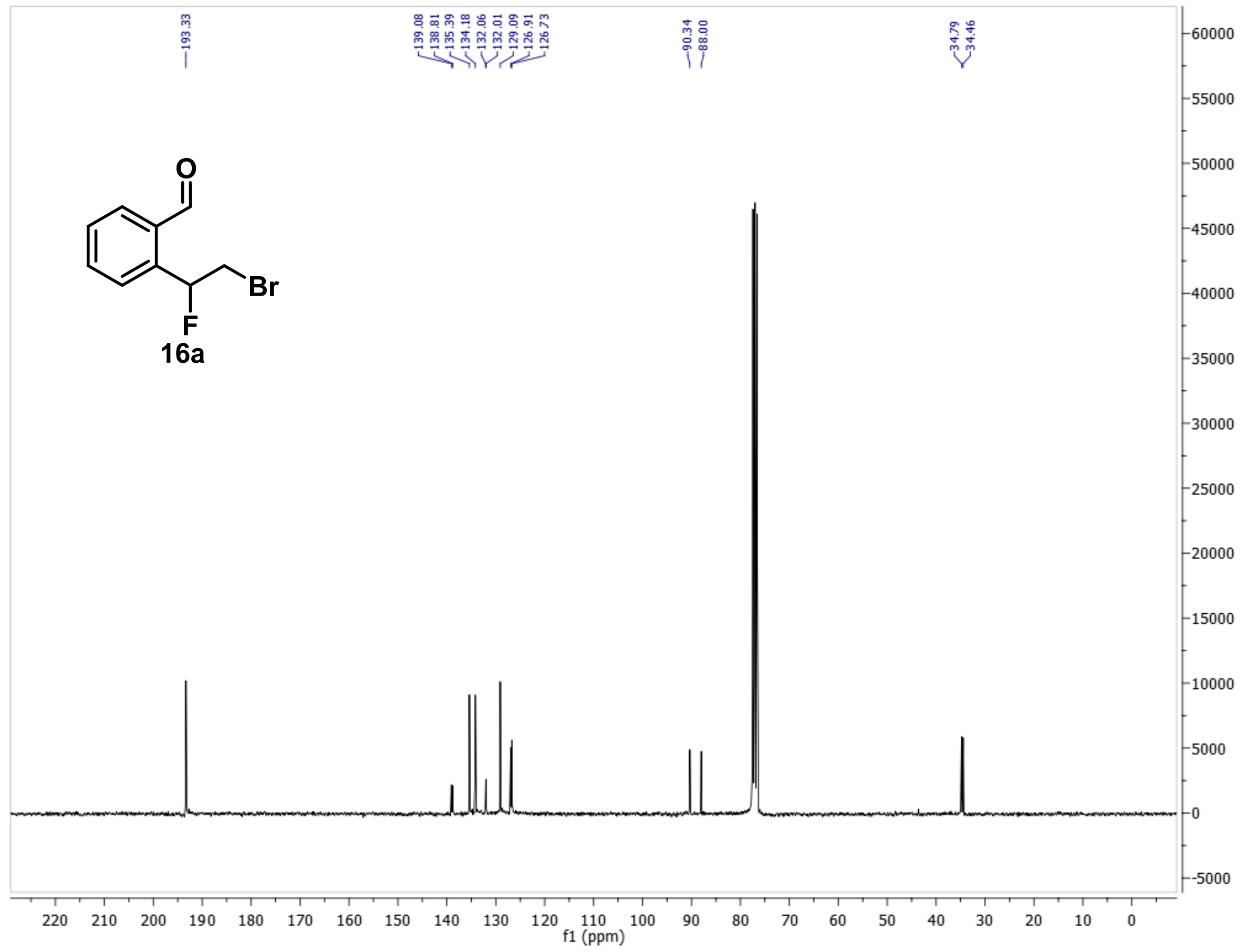




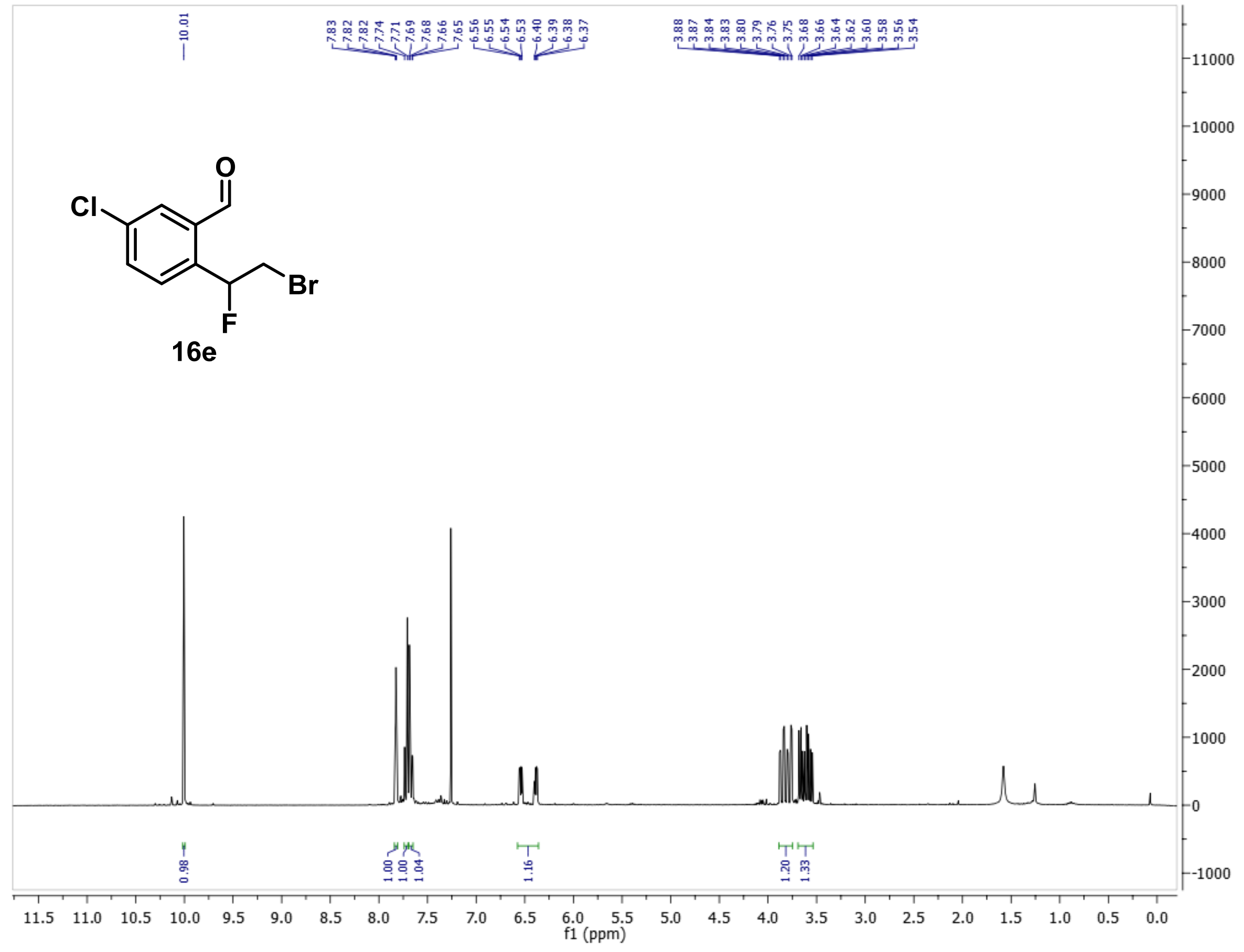




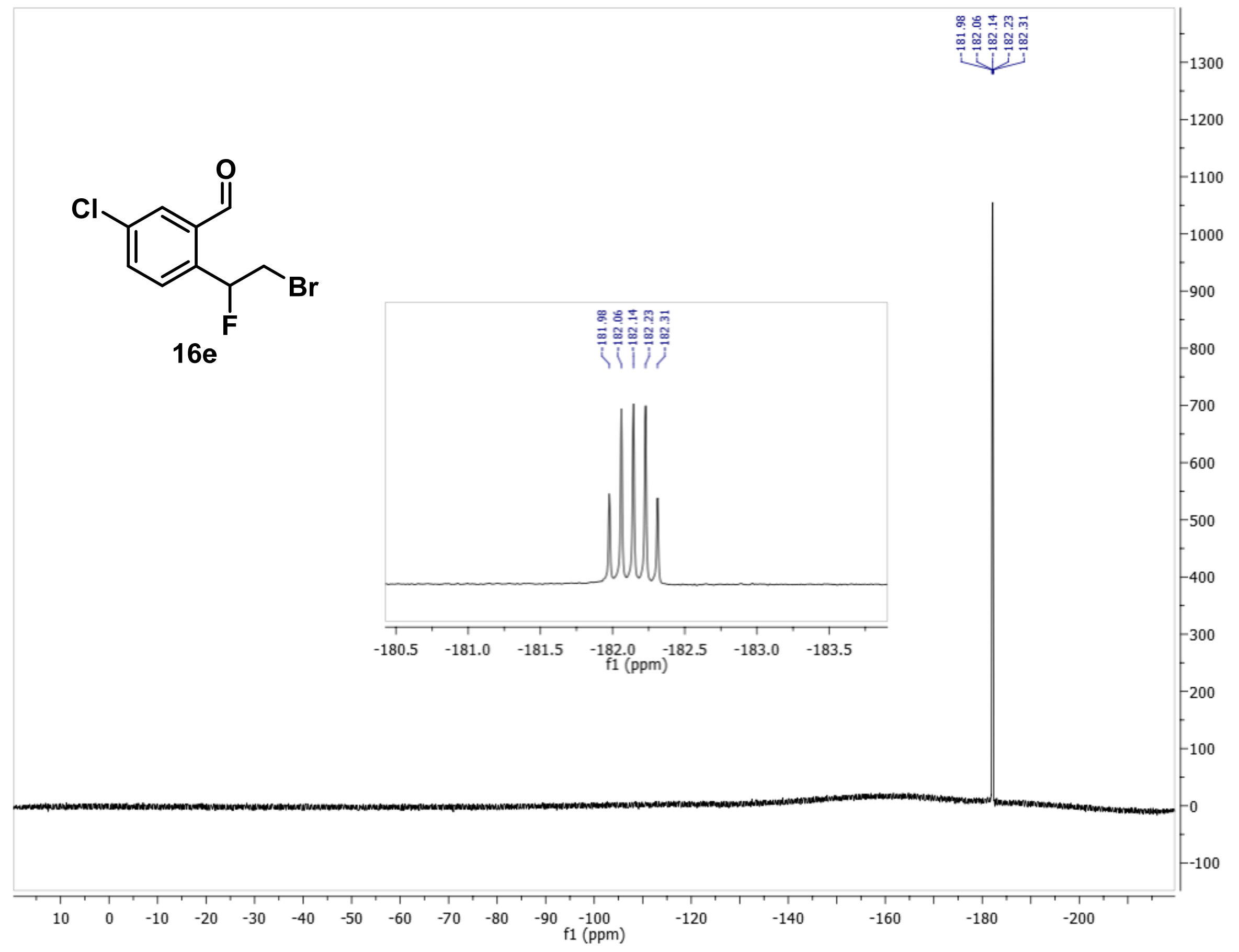




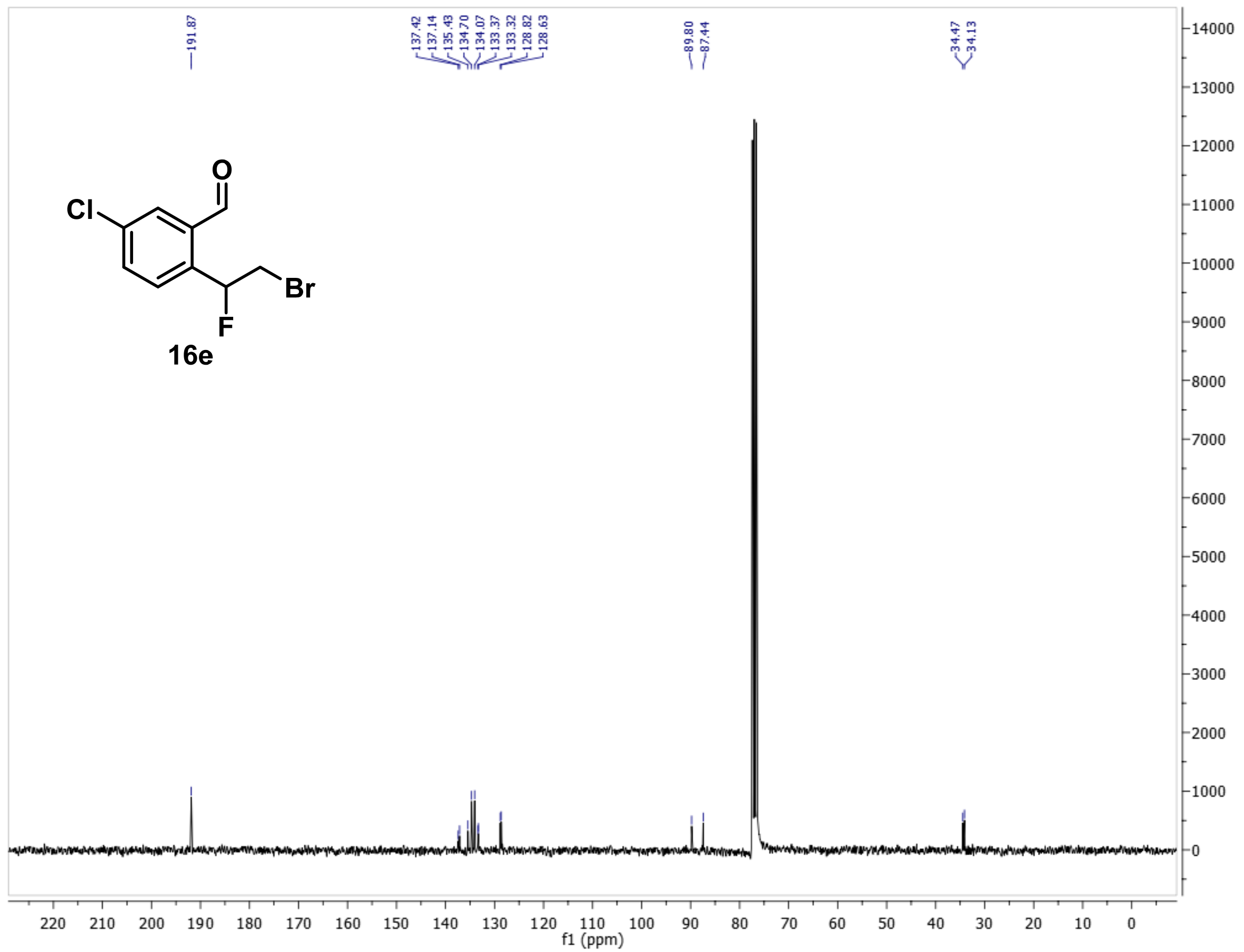




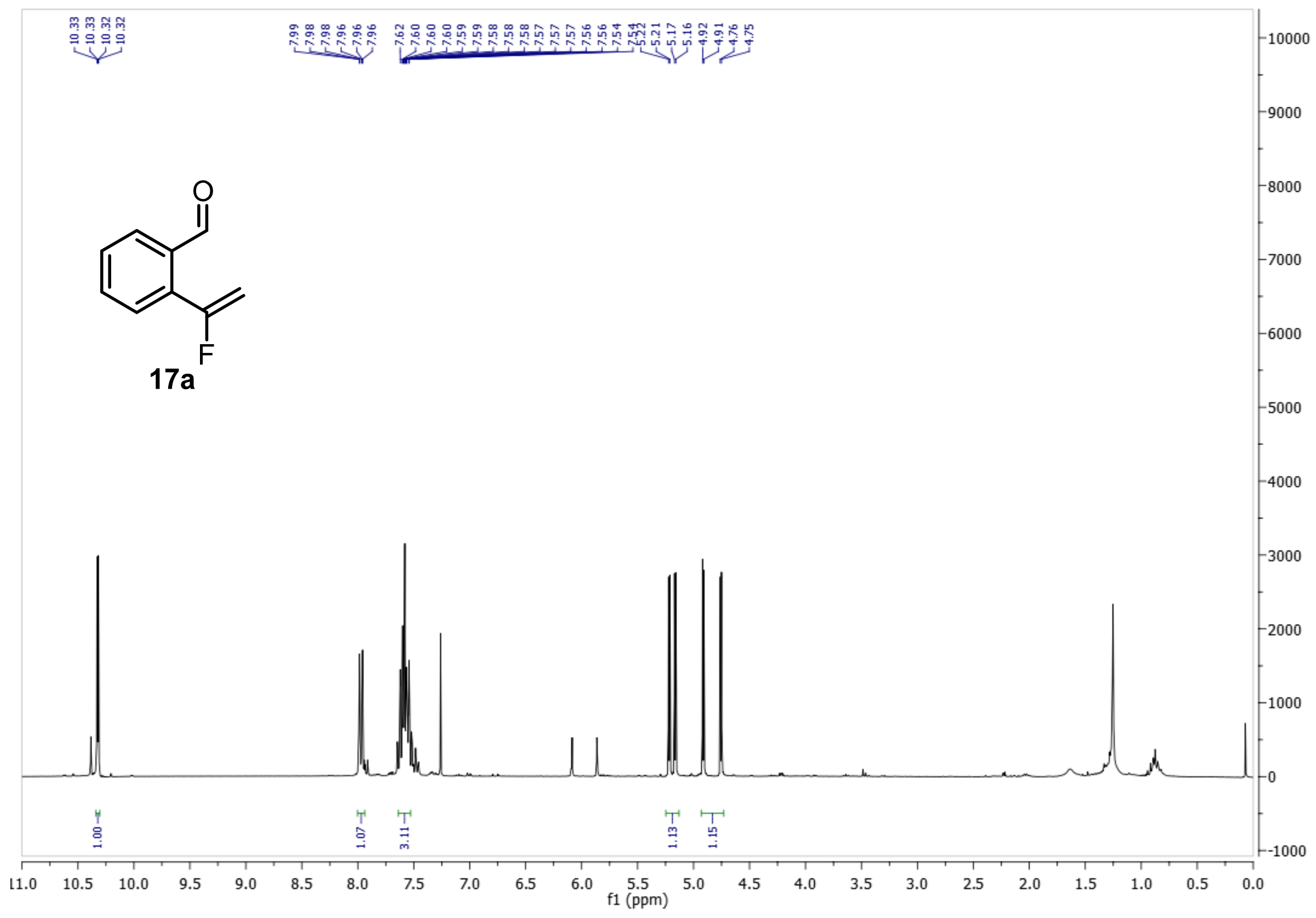




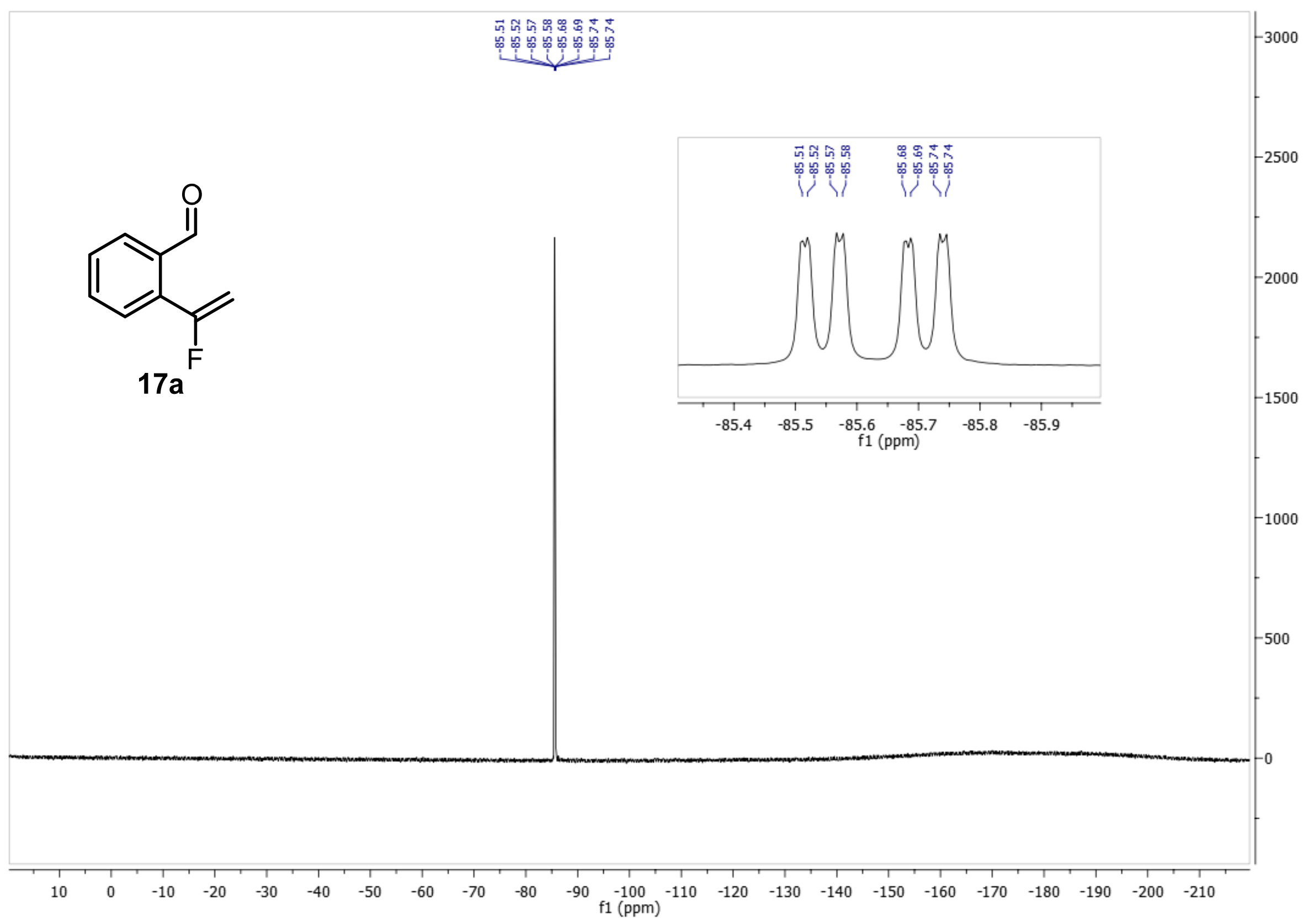




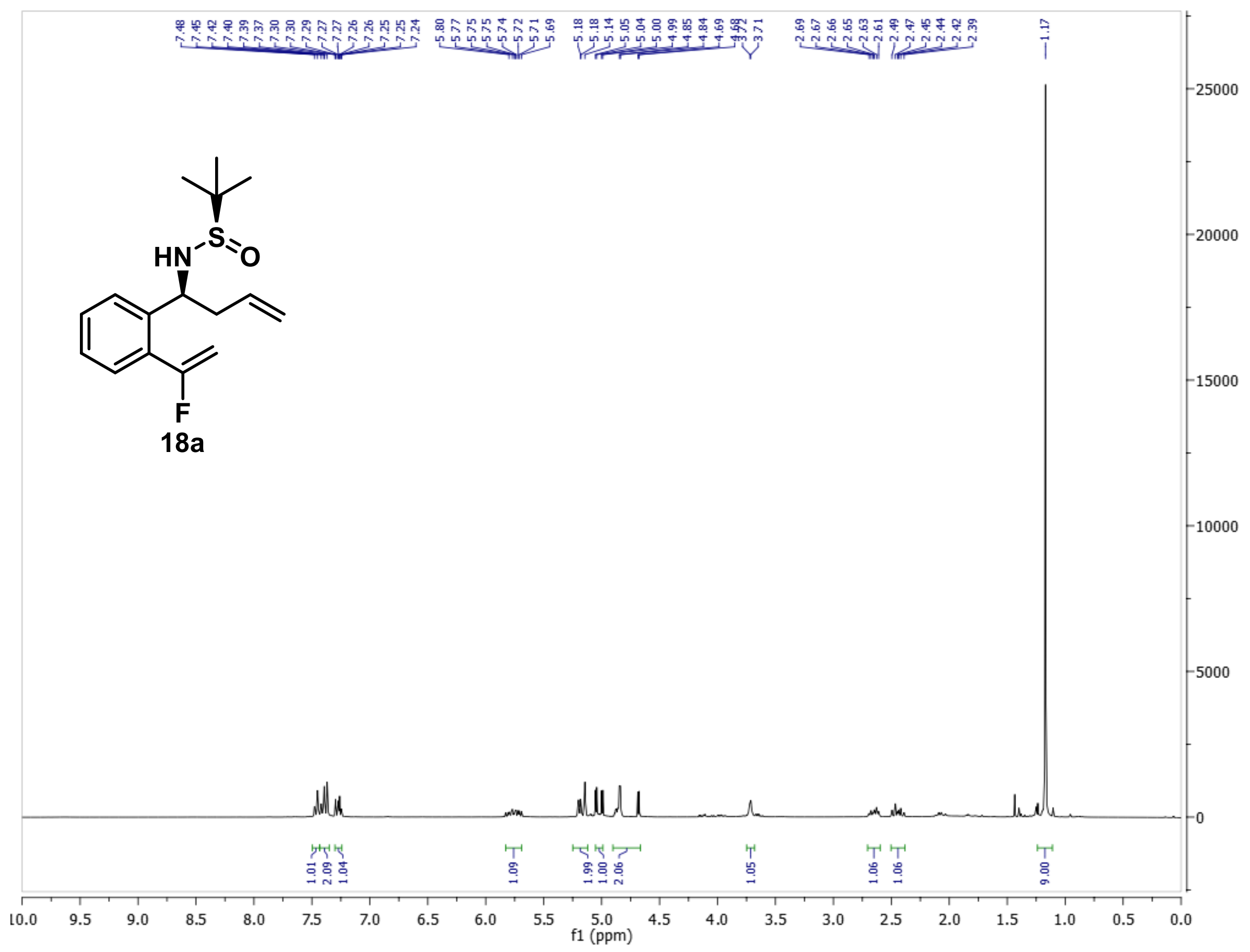




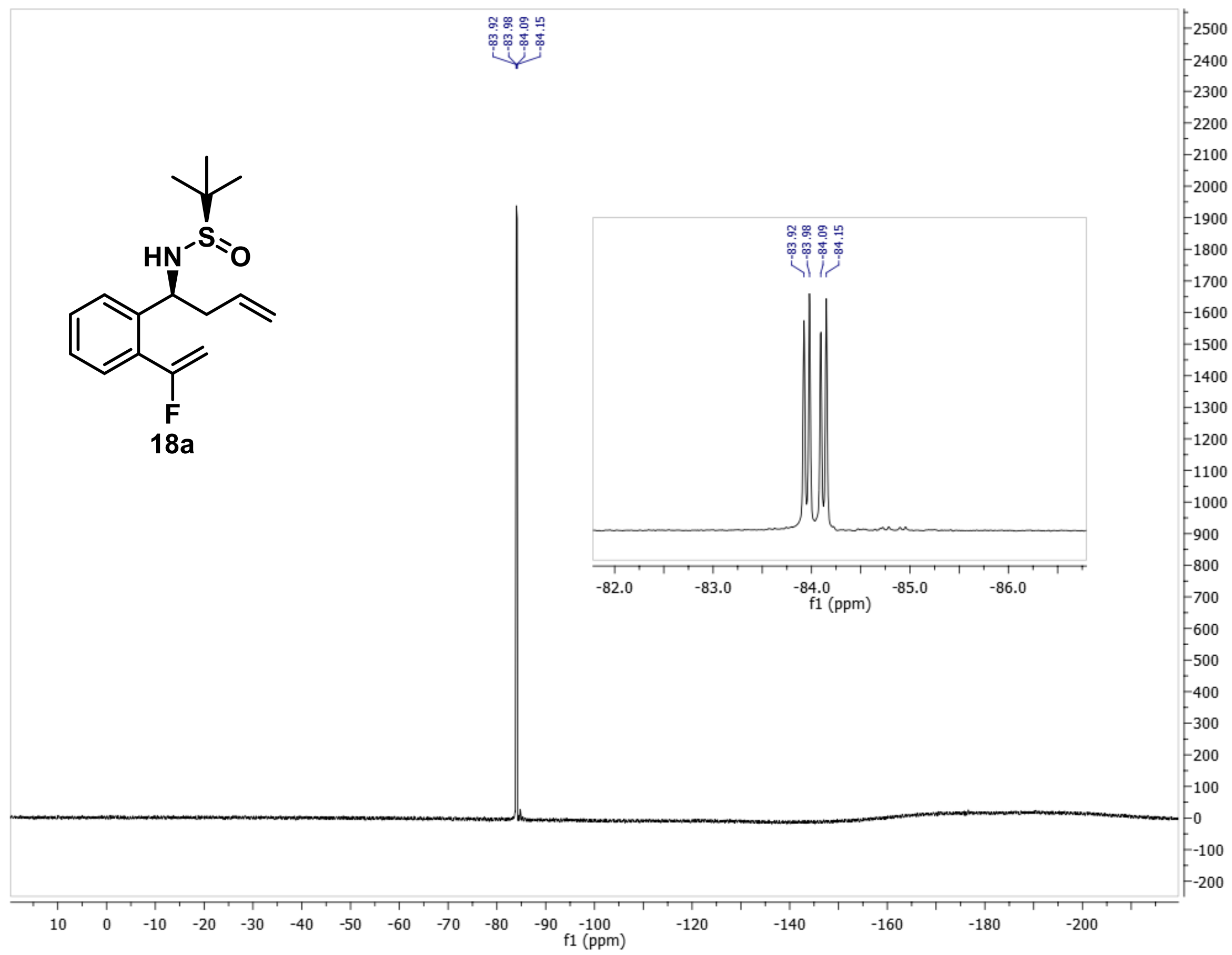




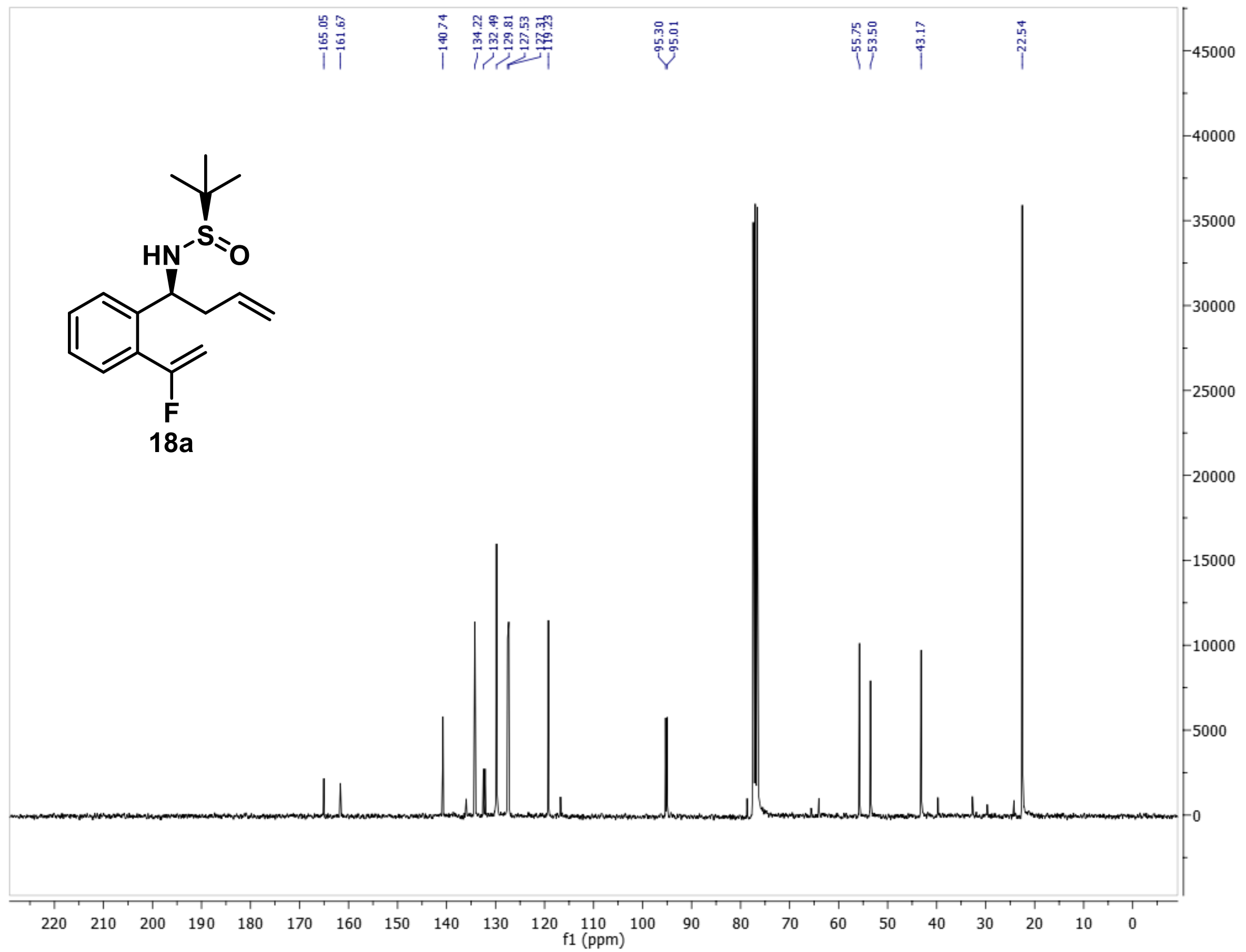




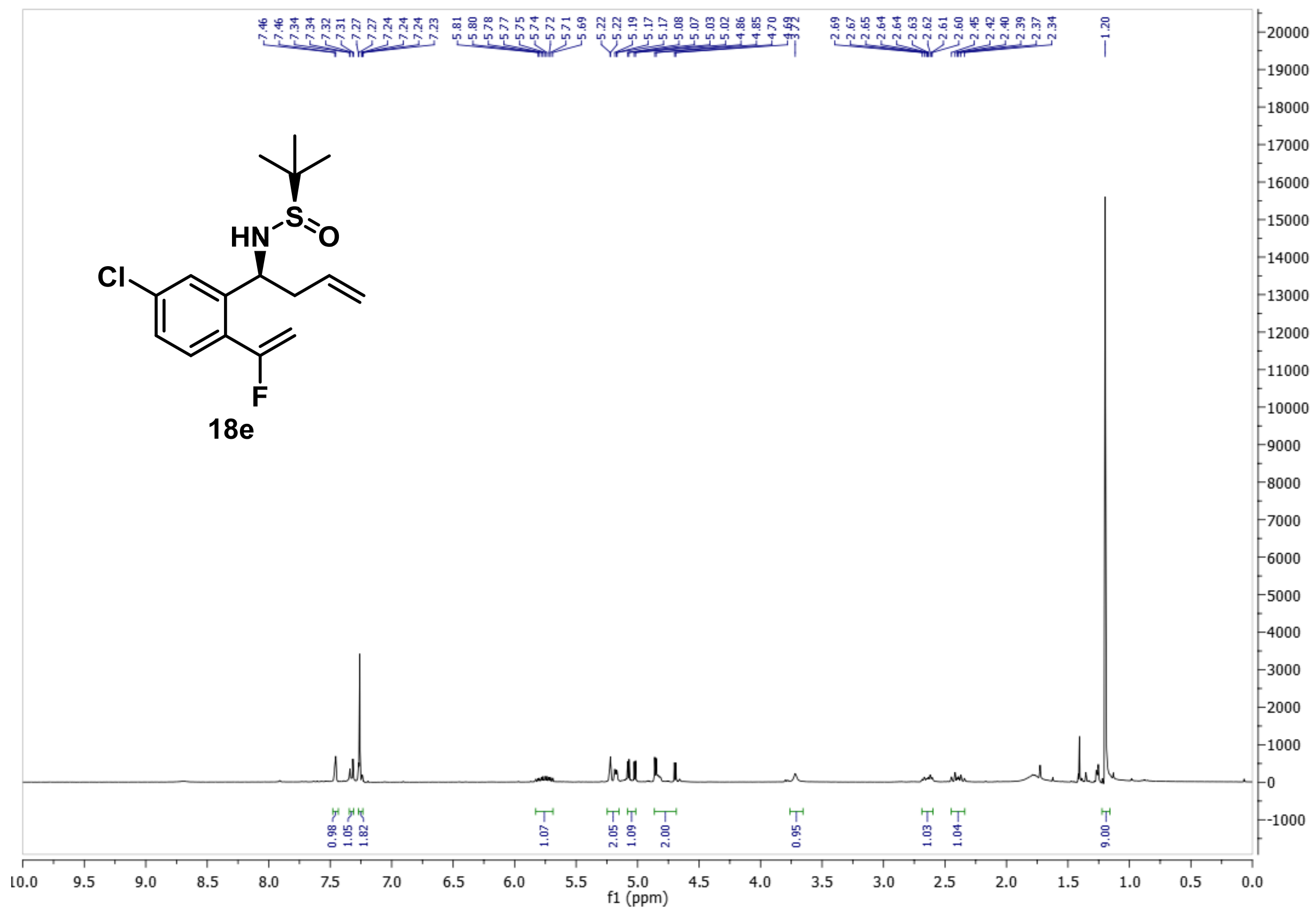




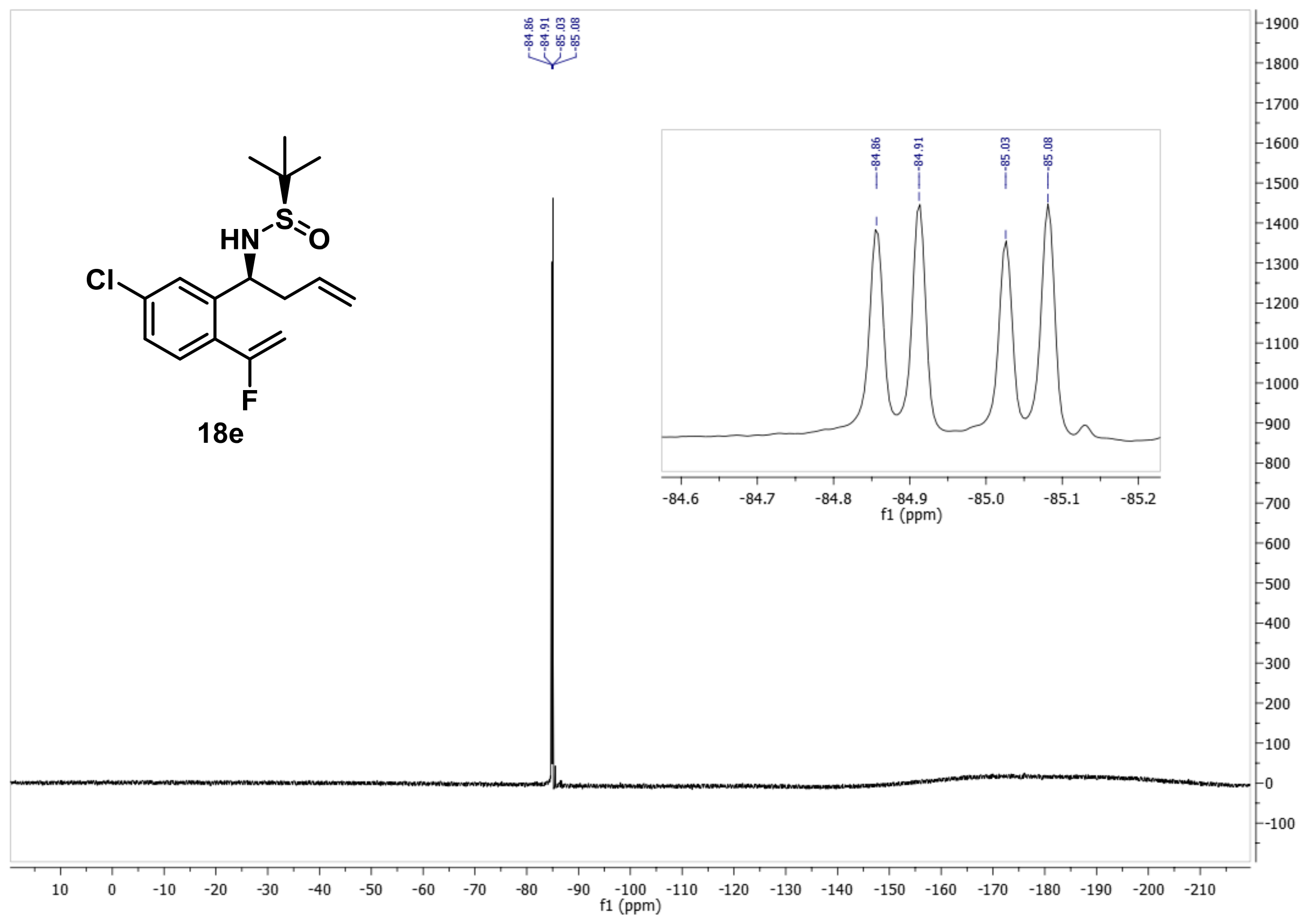




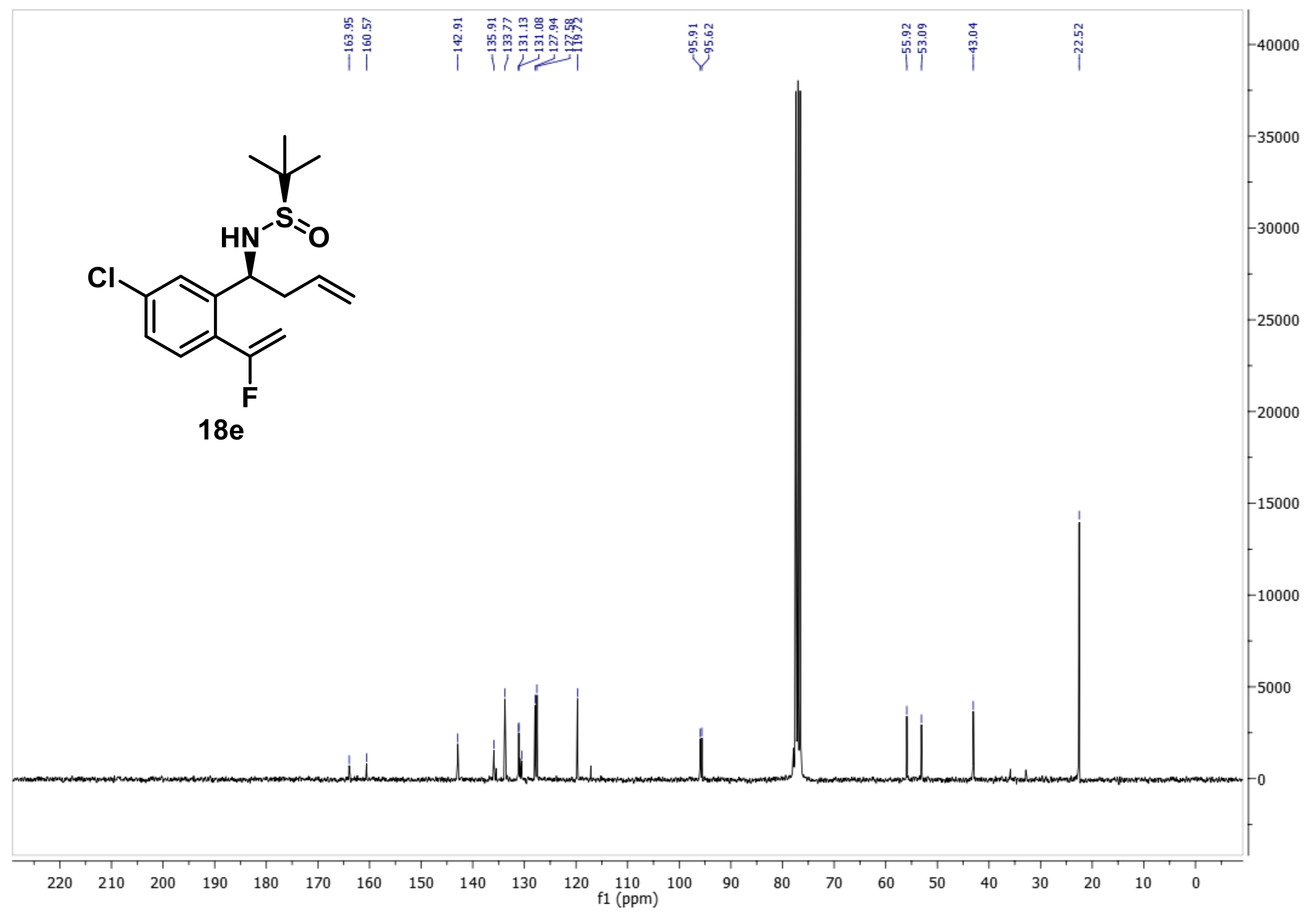




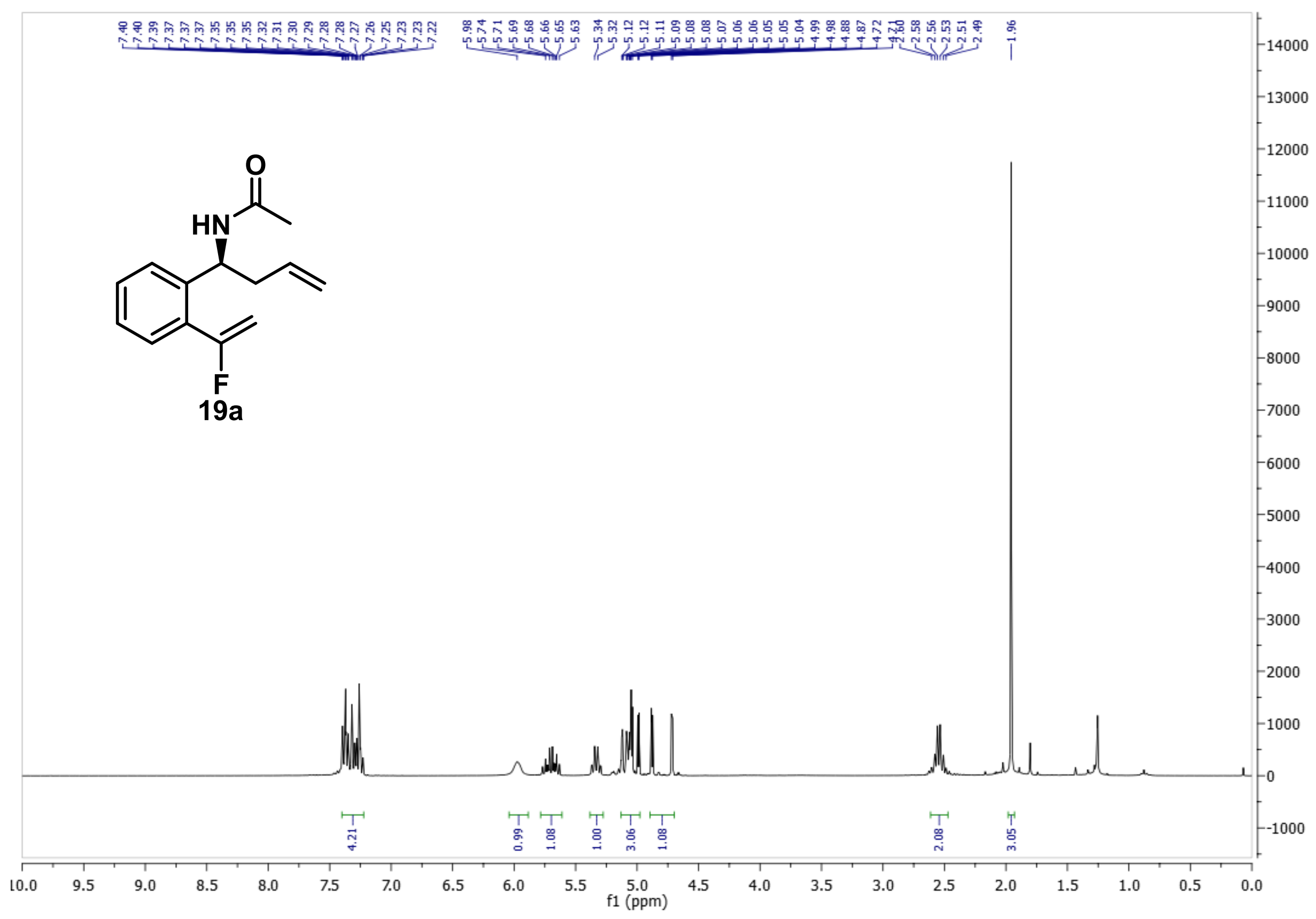




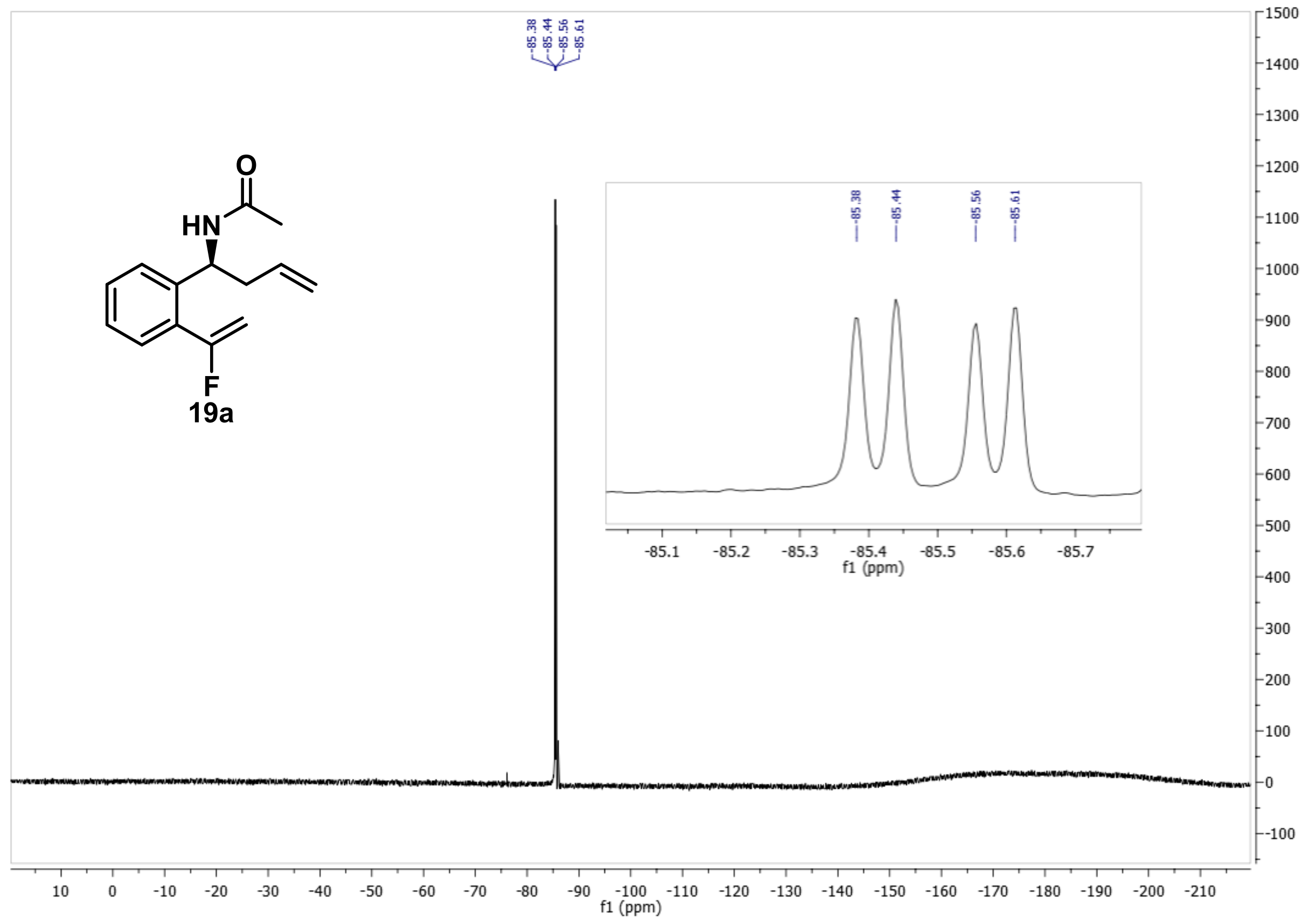




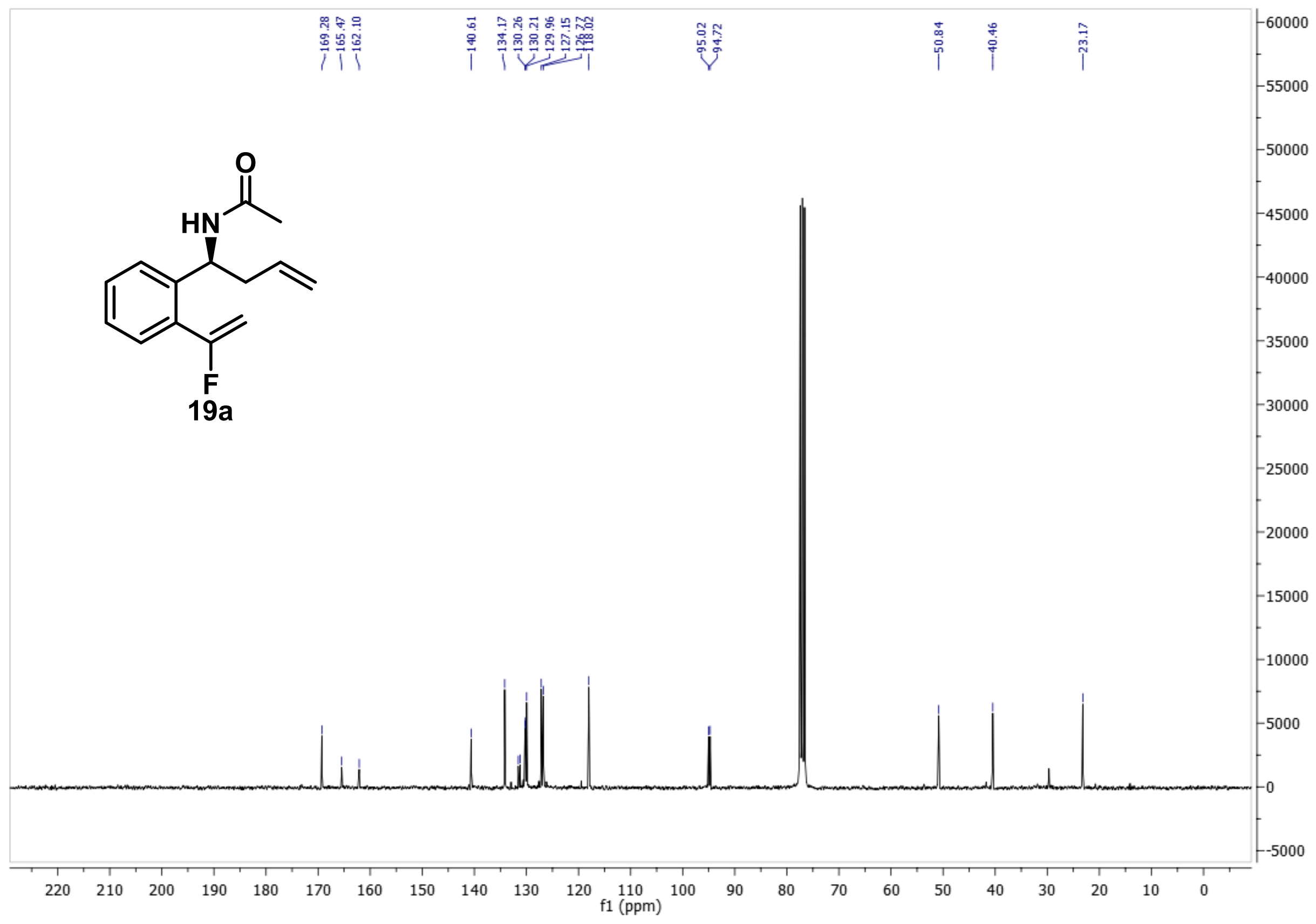




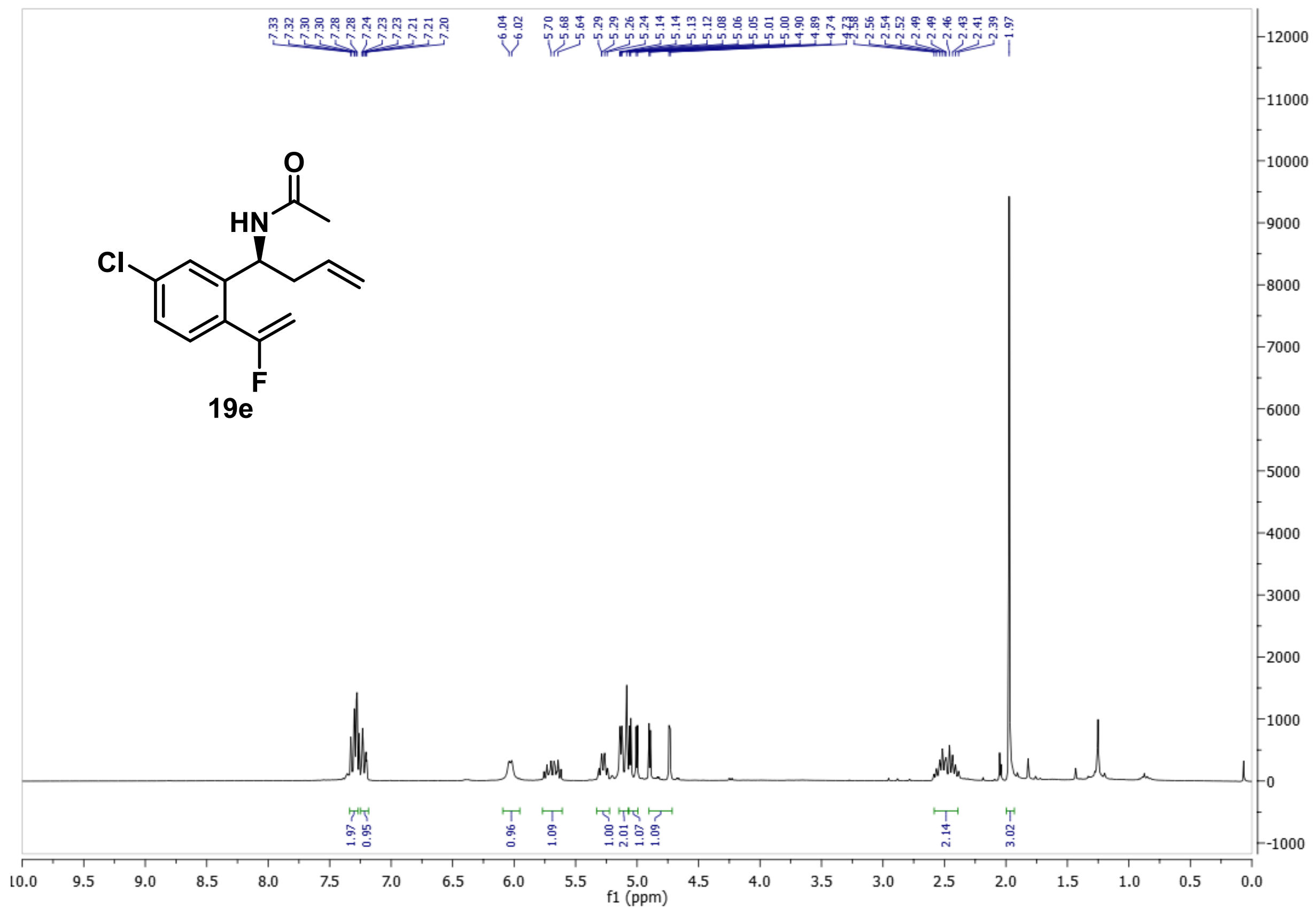




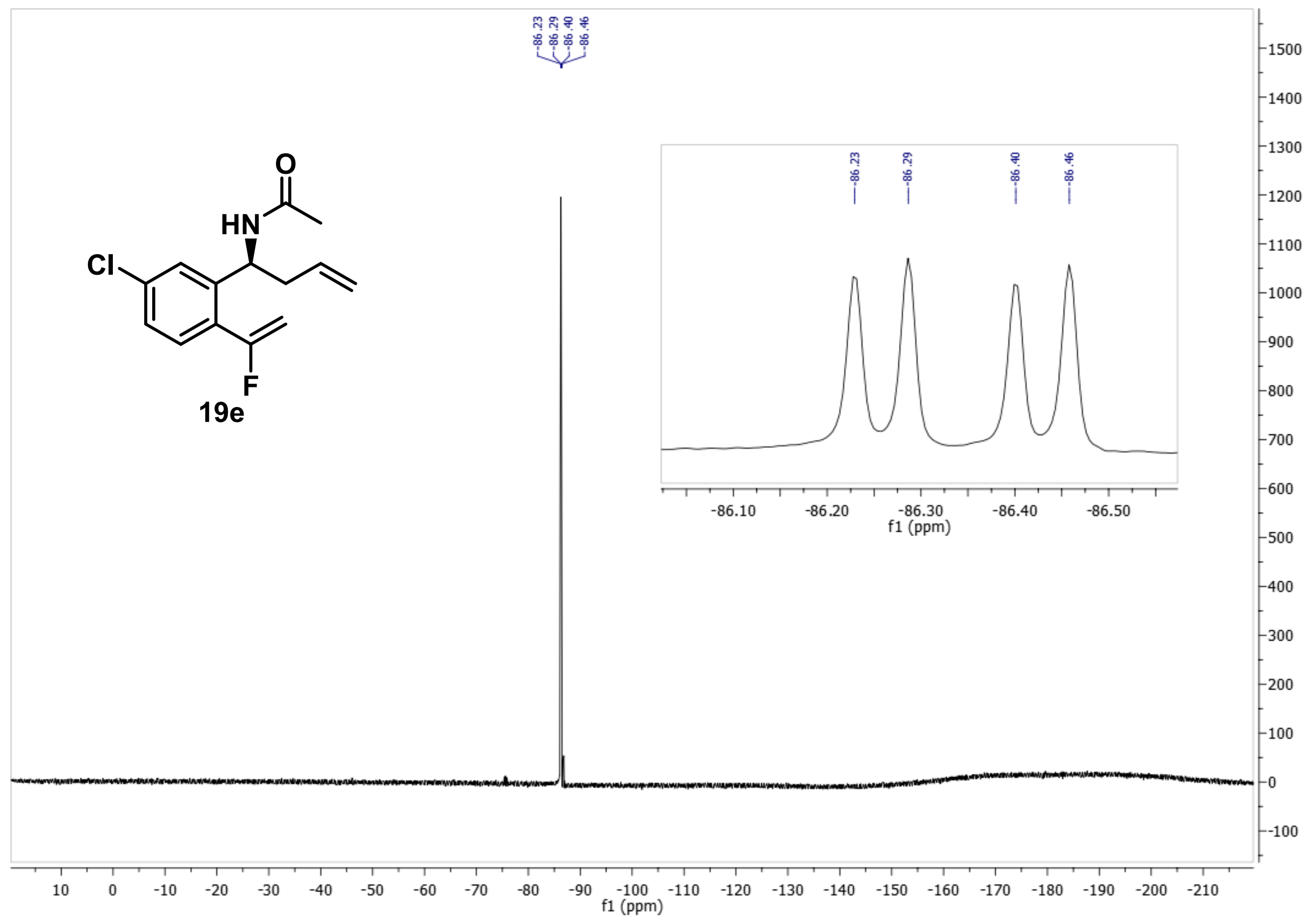




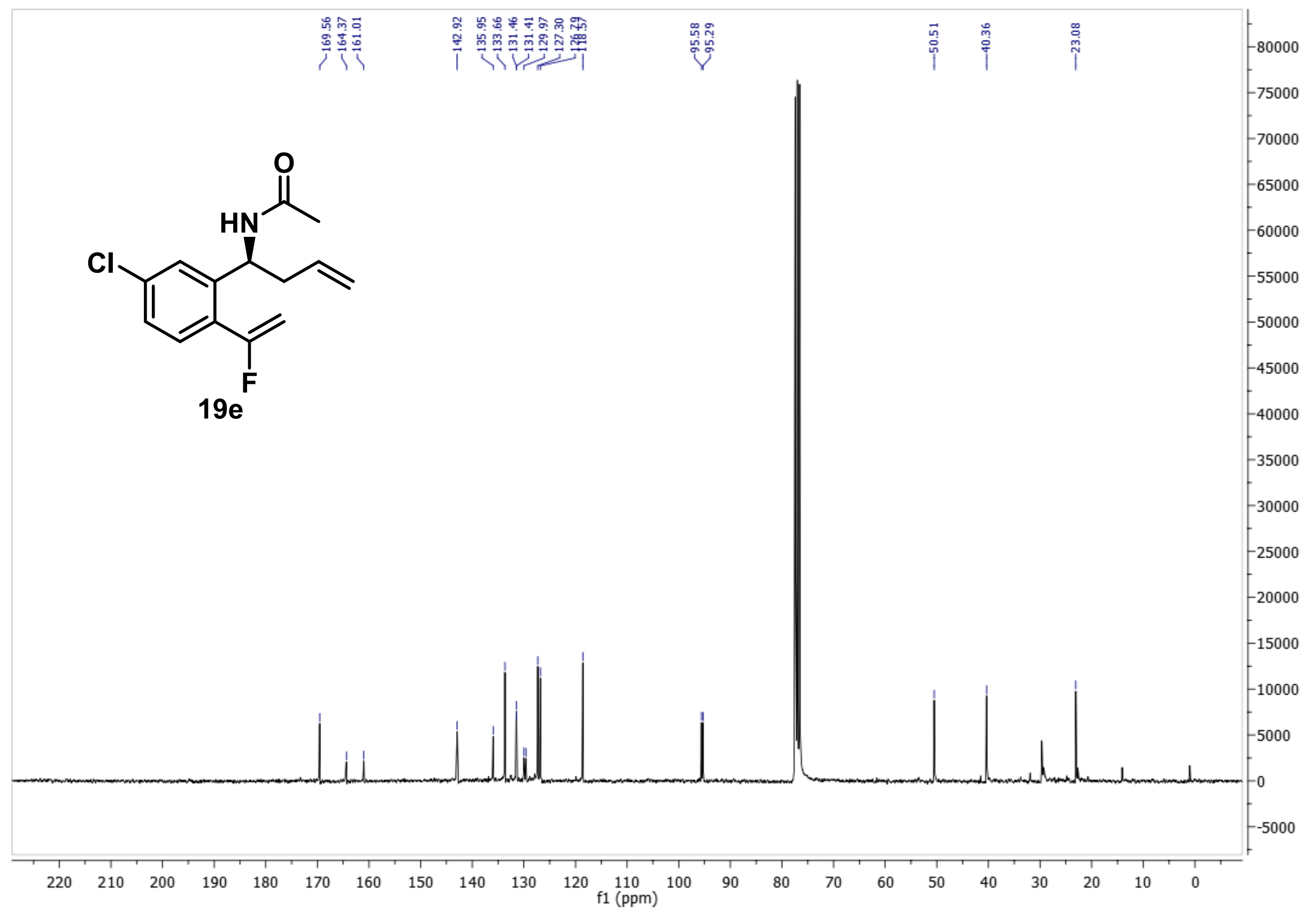




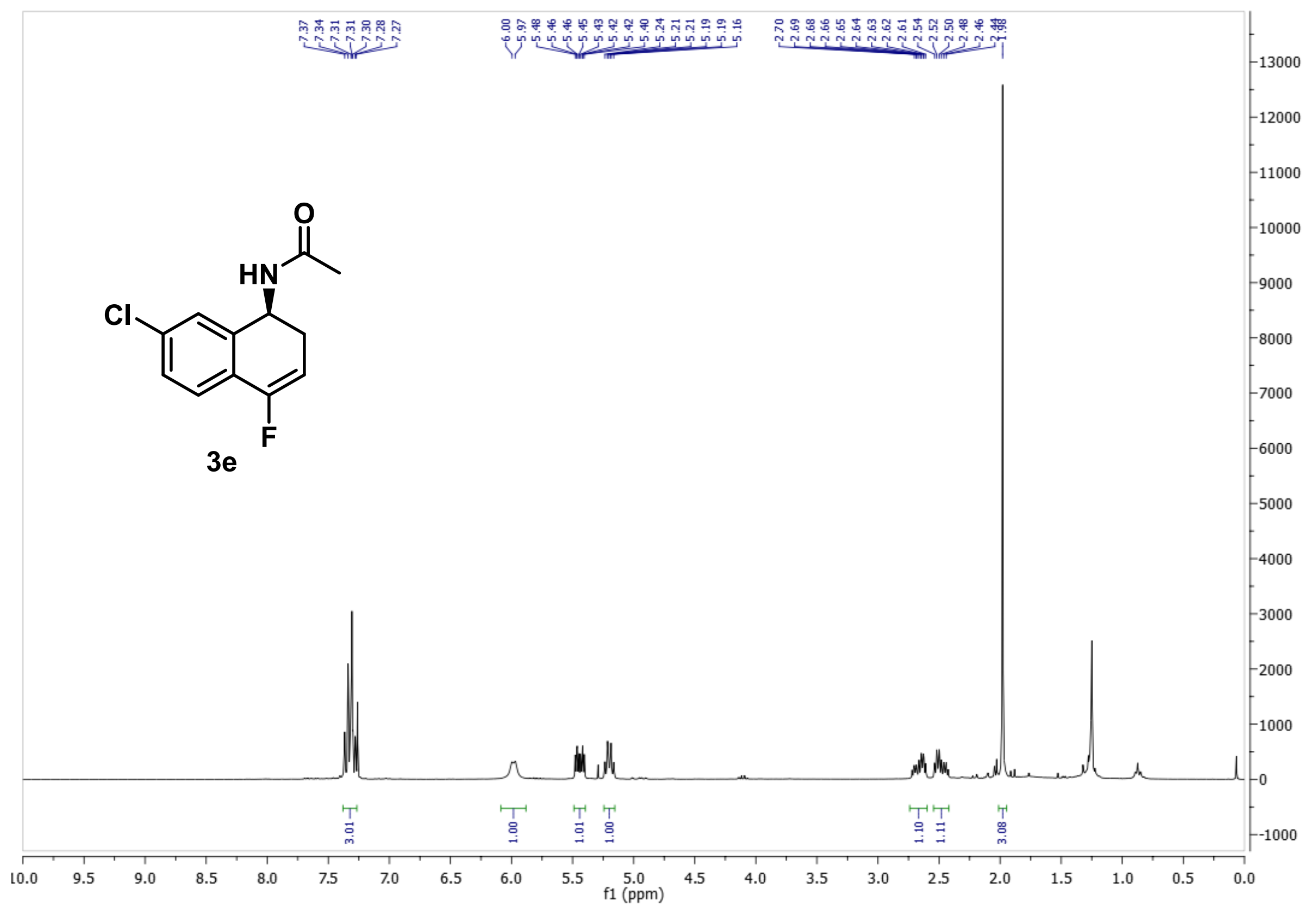




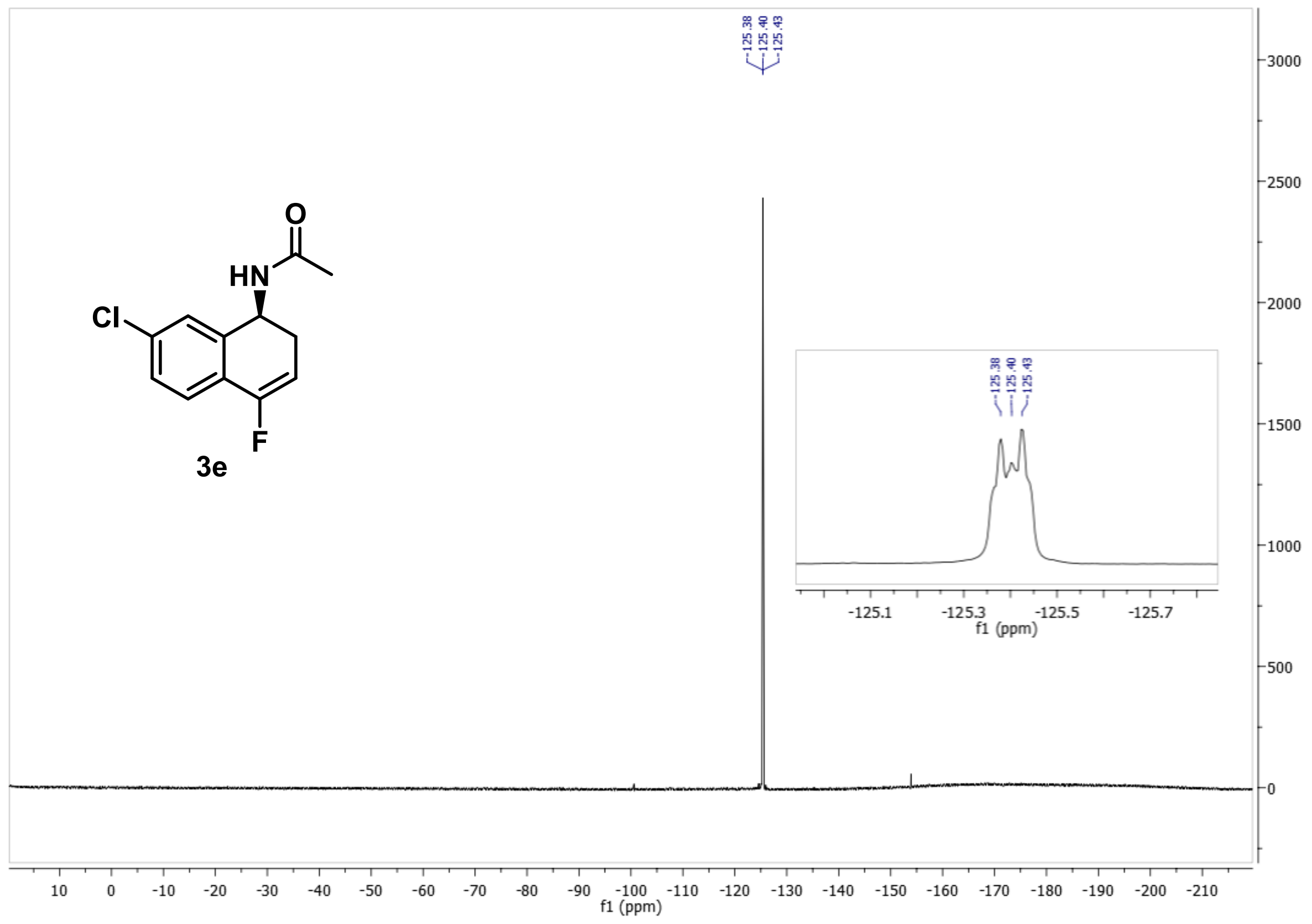




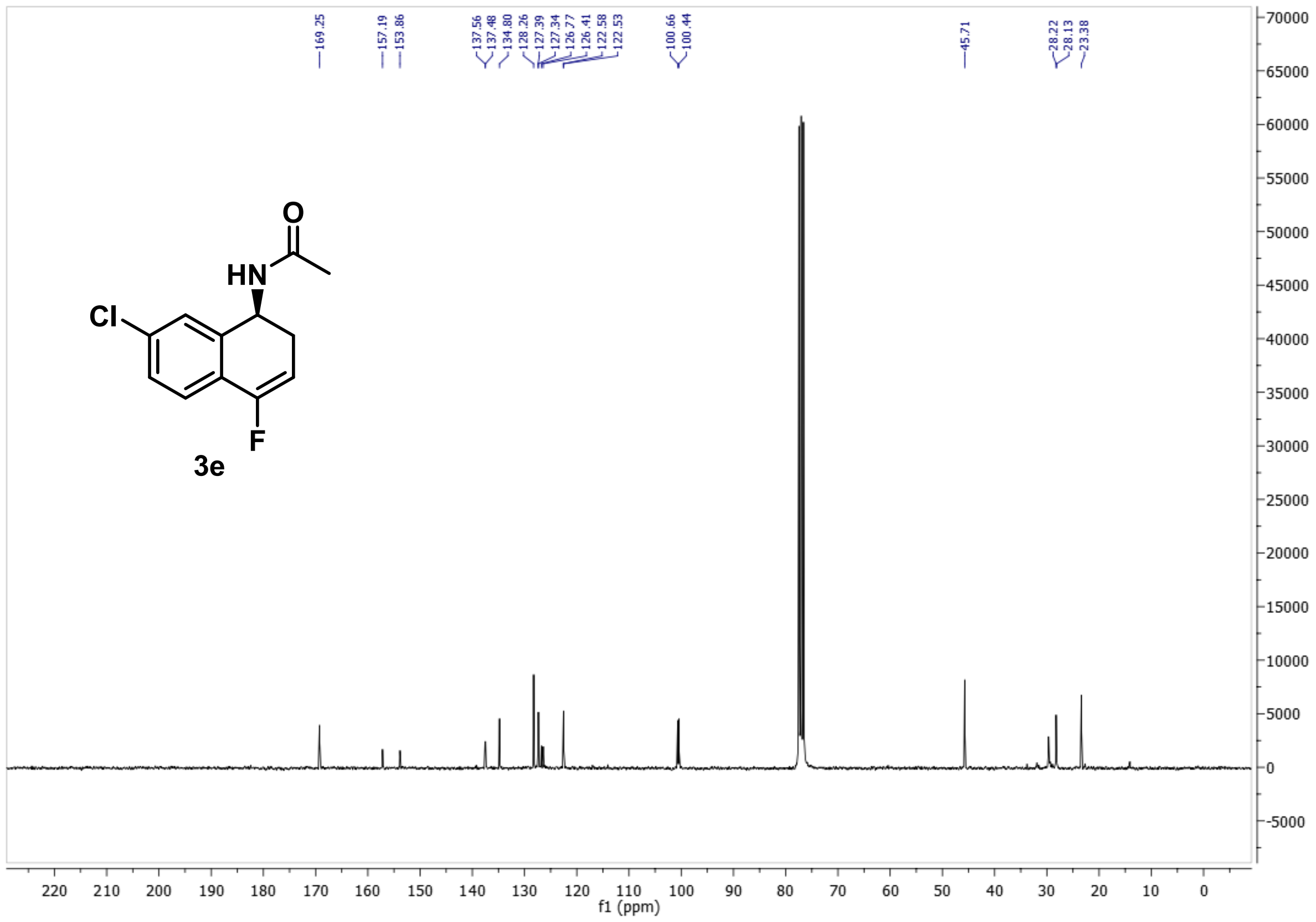




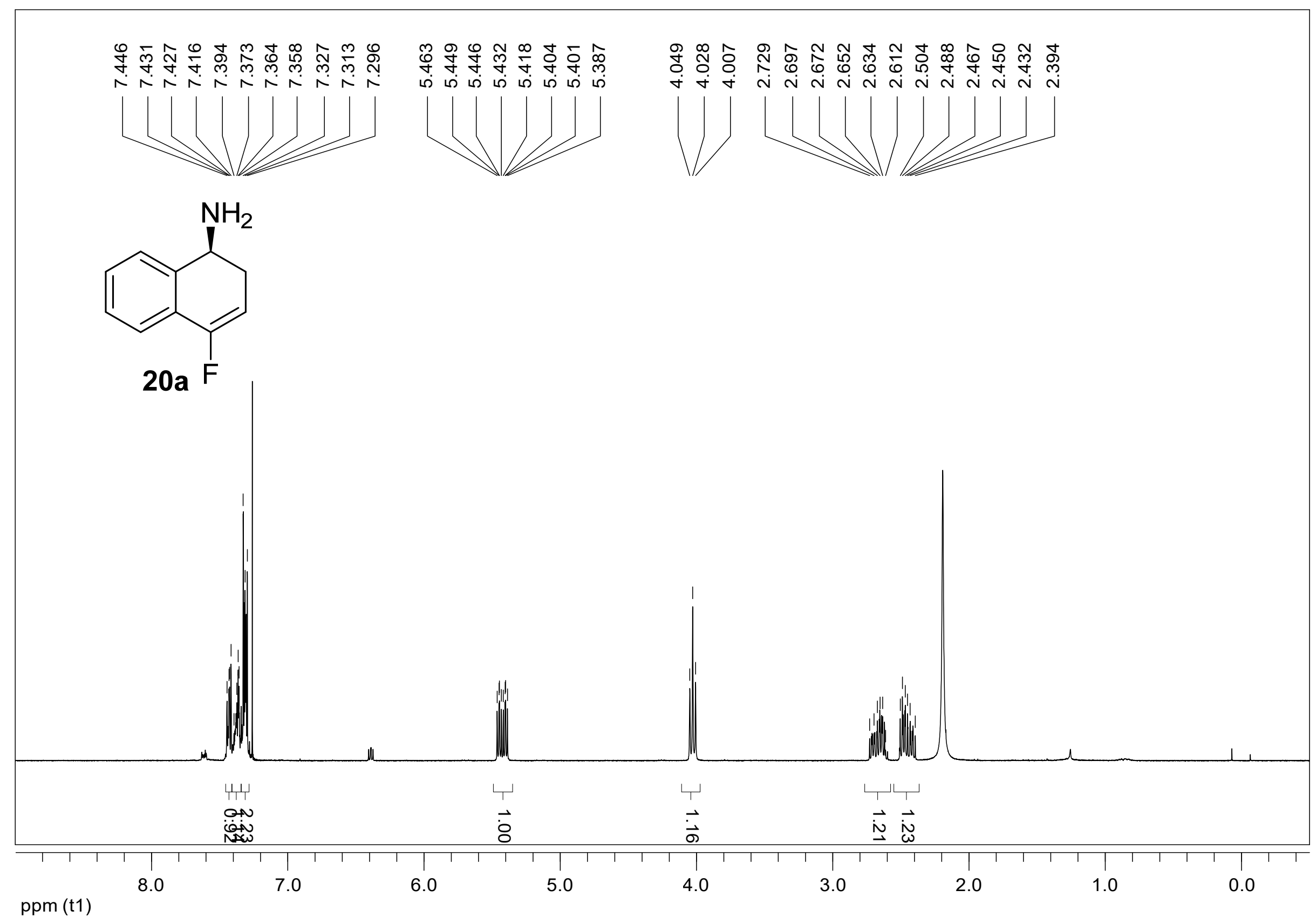




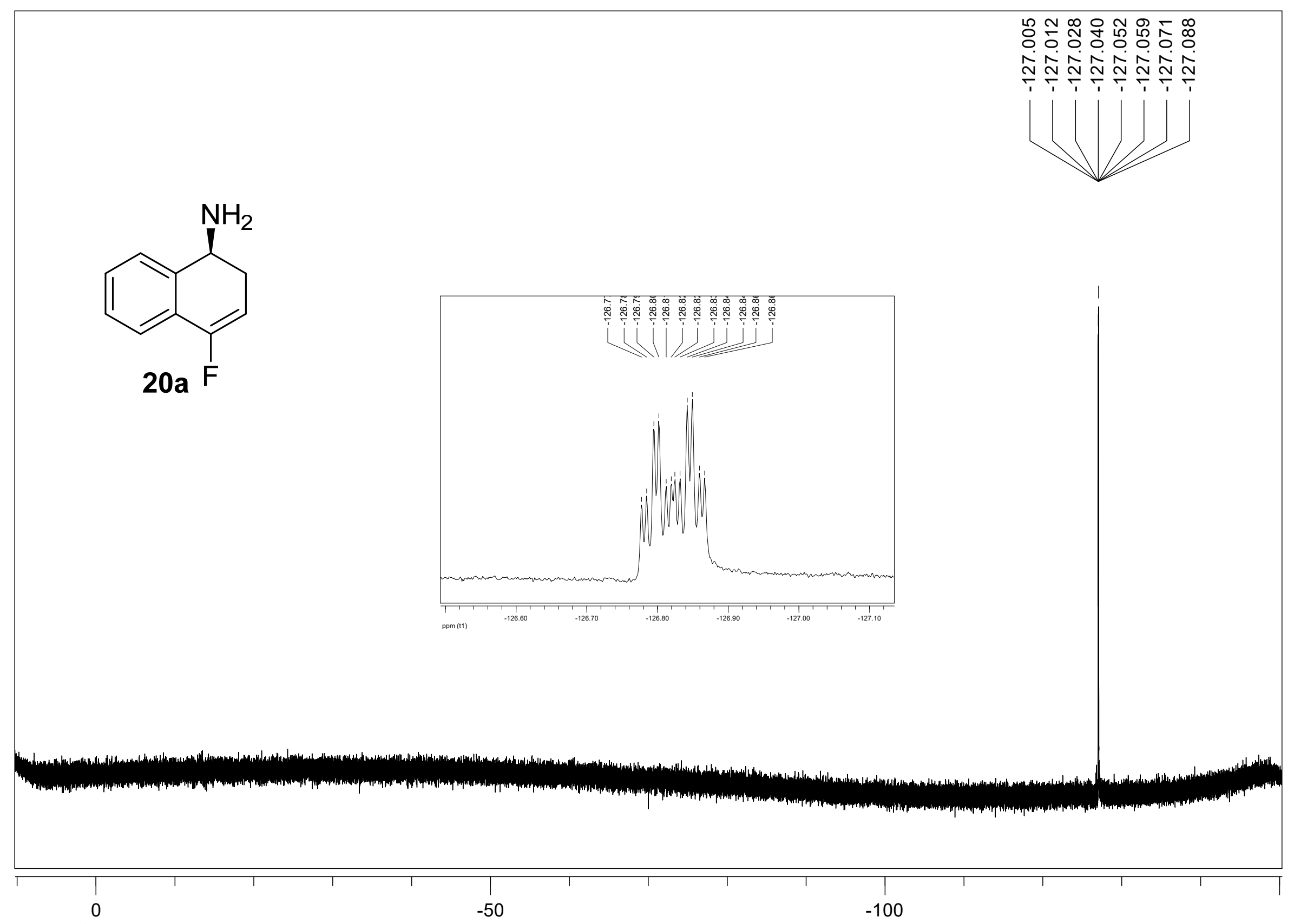

ppm (t1) 


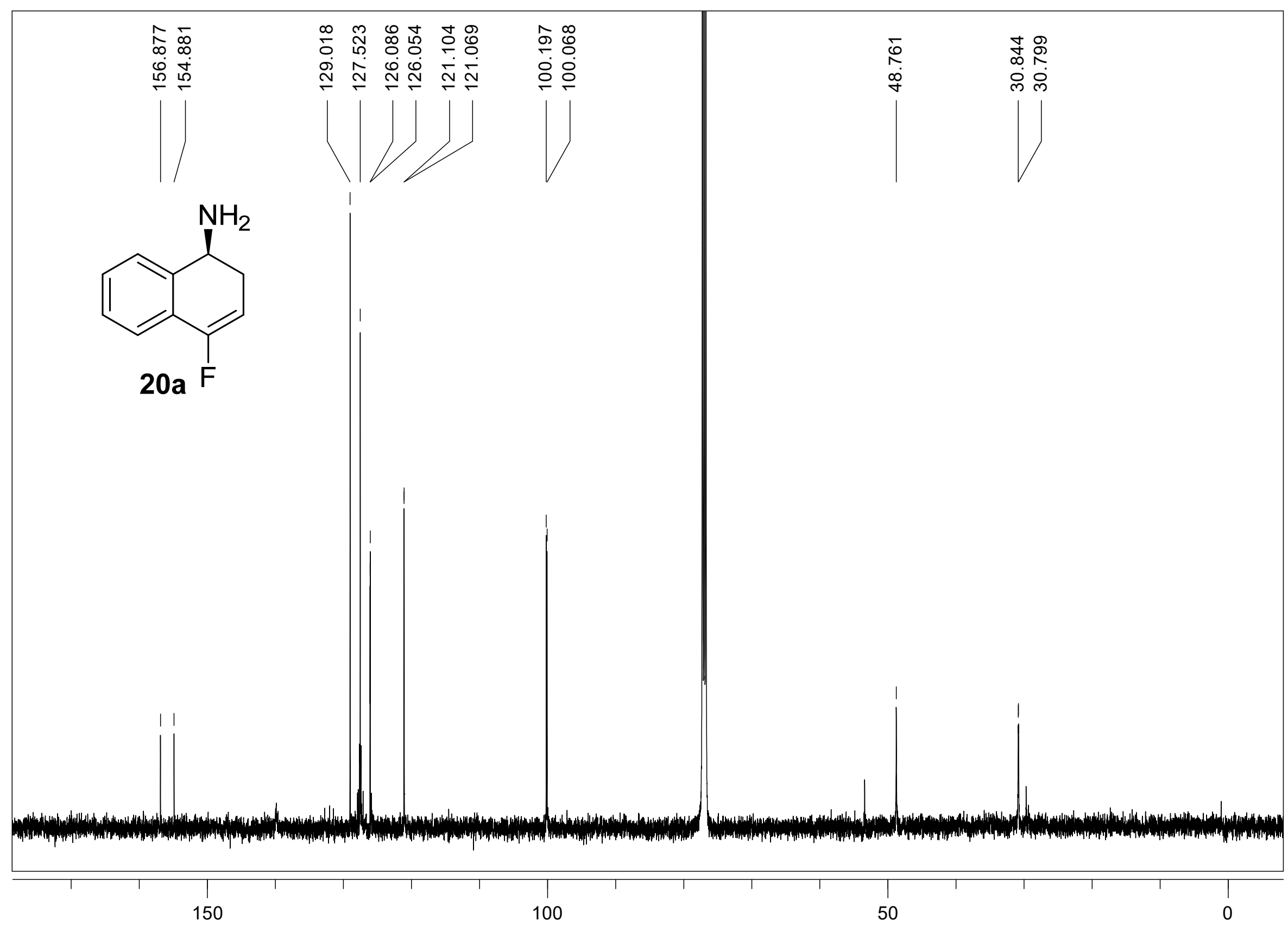

ppm (t1) 


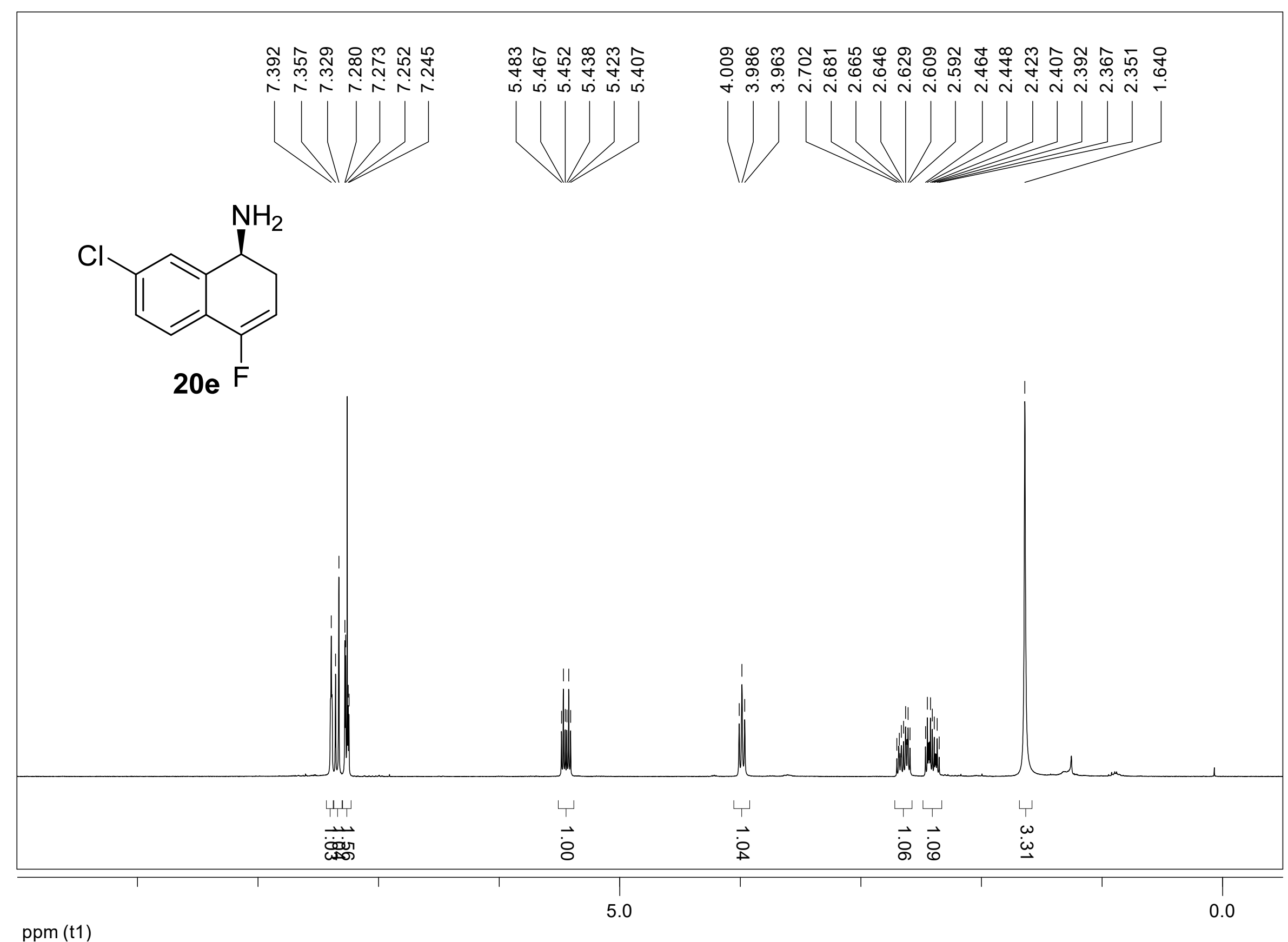

ppm (t1) 


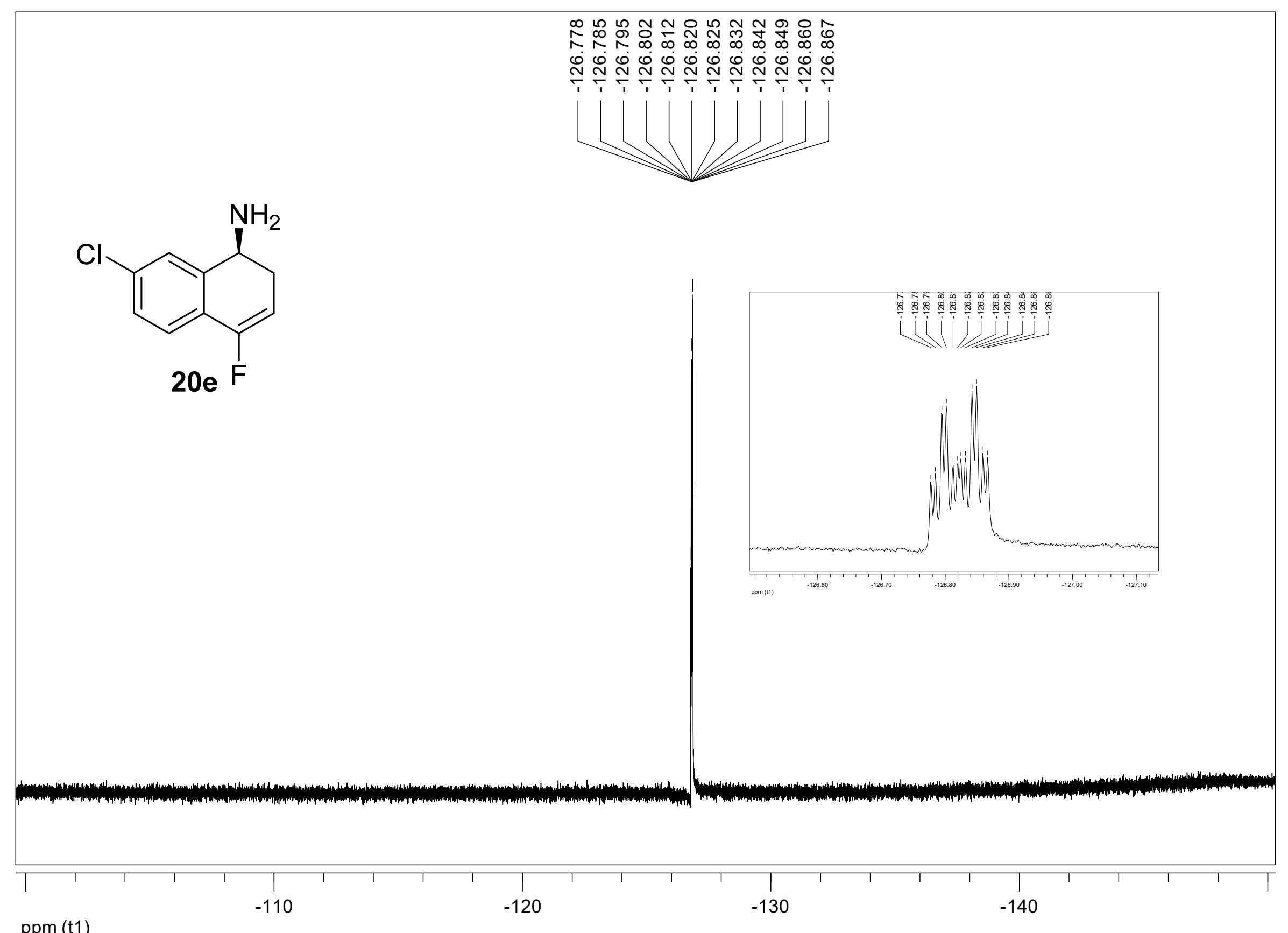

ppm (t1) 


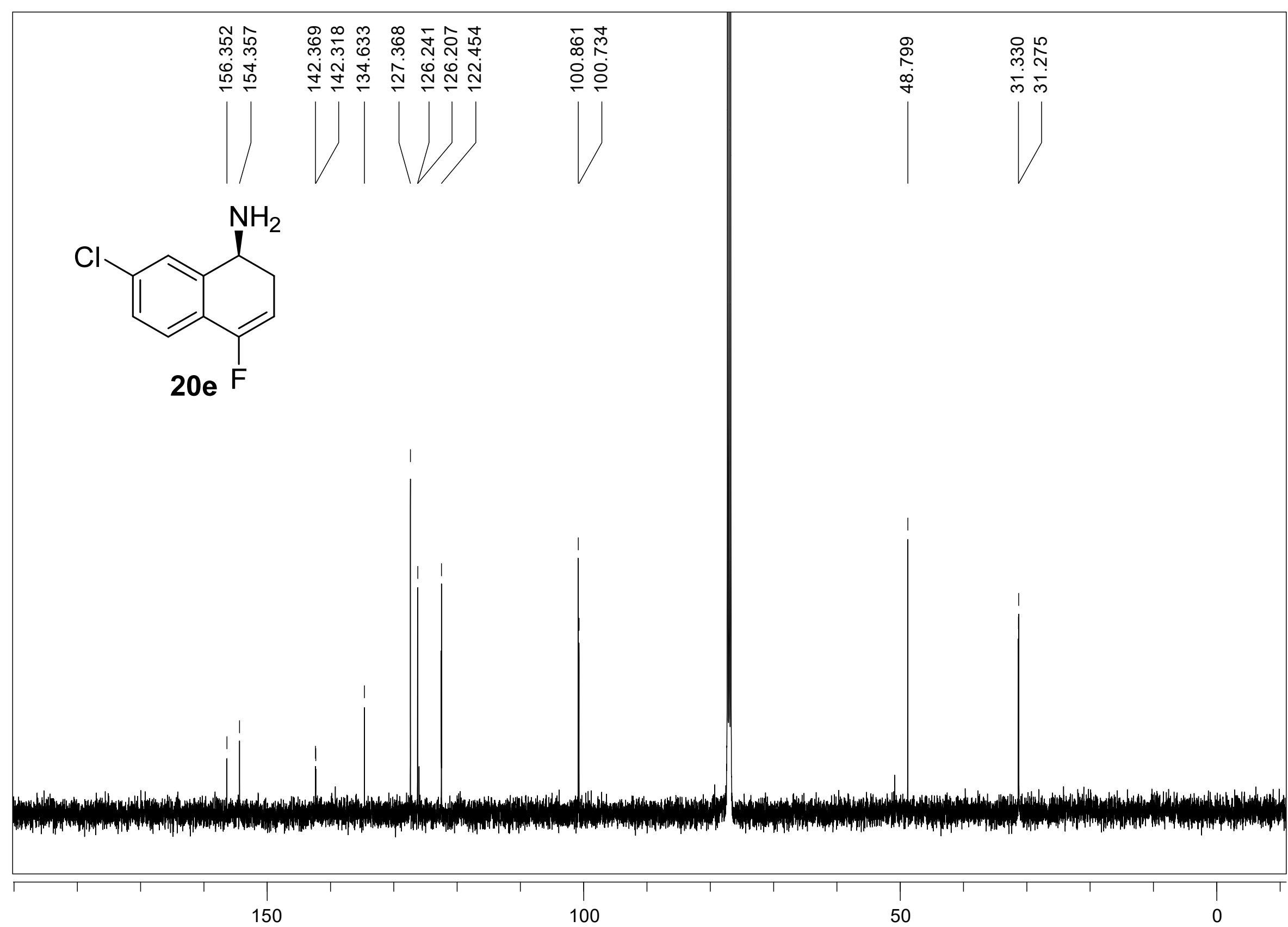

ppm (t1) 
Chiralcel OD-H, hexane:i-PrOH (95:5), $1 \mathrm{~mL} / \mathrm{min}$
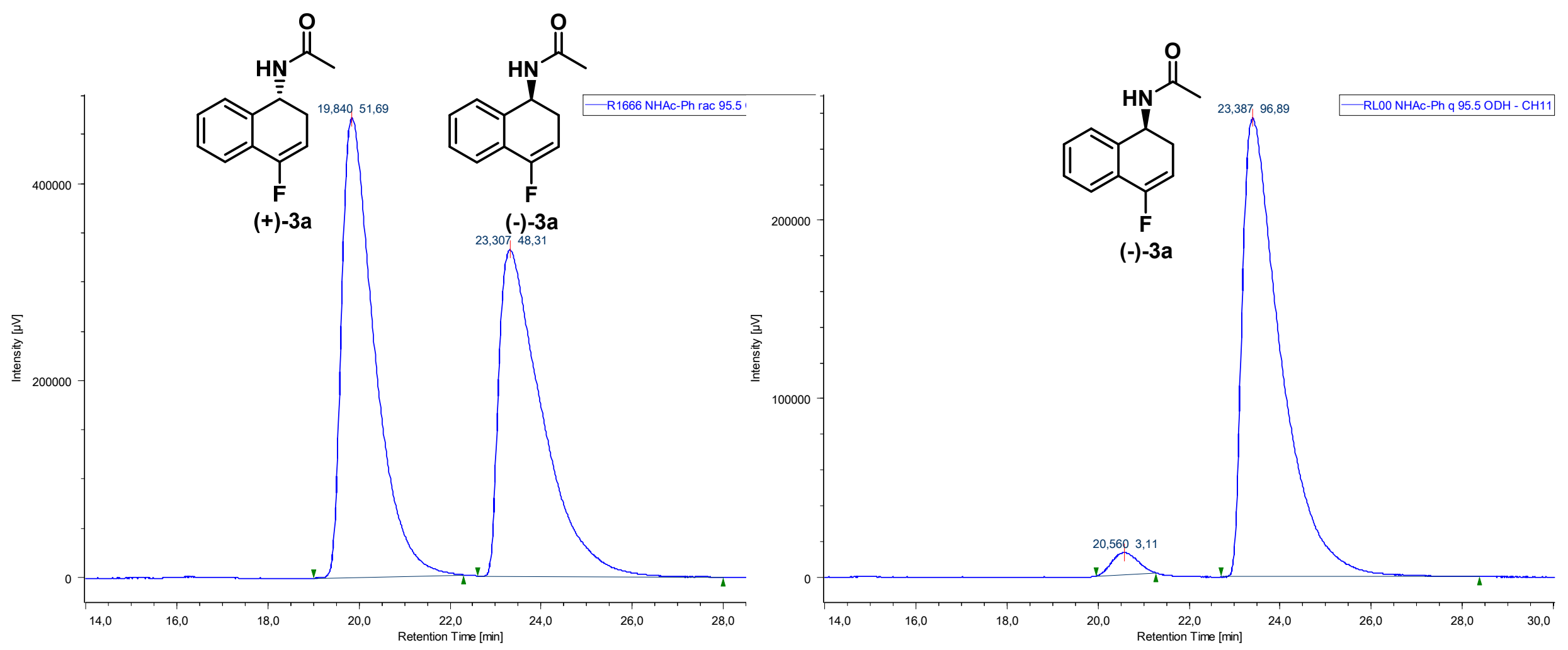


\section{Chiralcel OD-H, hexane:i-PrOH (95:5), $1 \mathrm{~mL} / \mathrm{min}$}
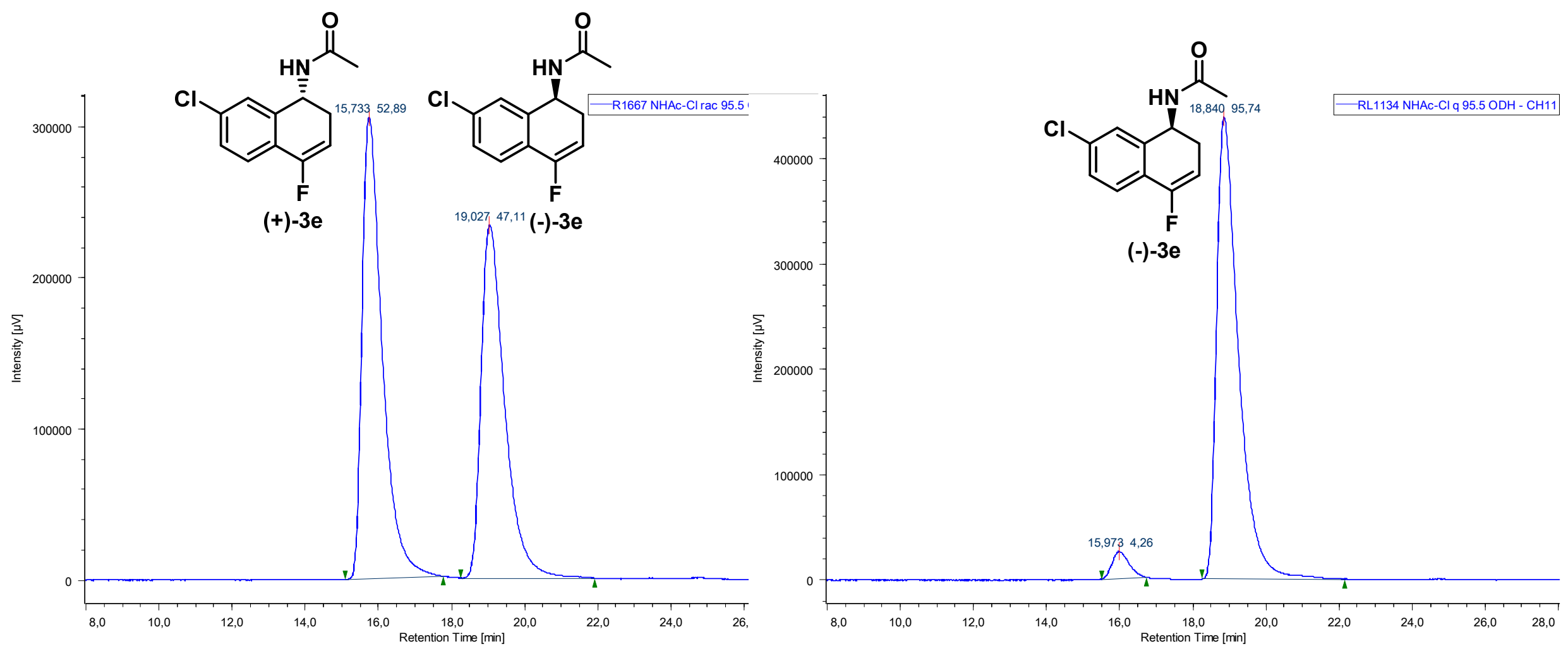
Chiralcel OD-H, hexane:i-PrOH (99:1), $1 \mathrm{~mL} / \mathrm{min}$
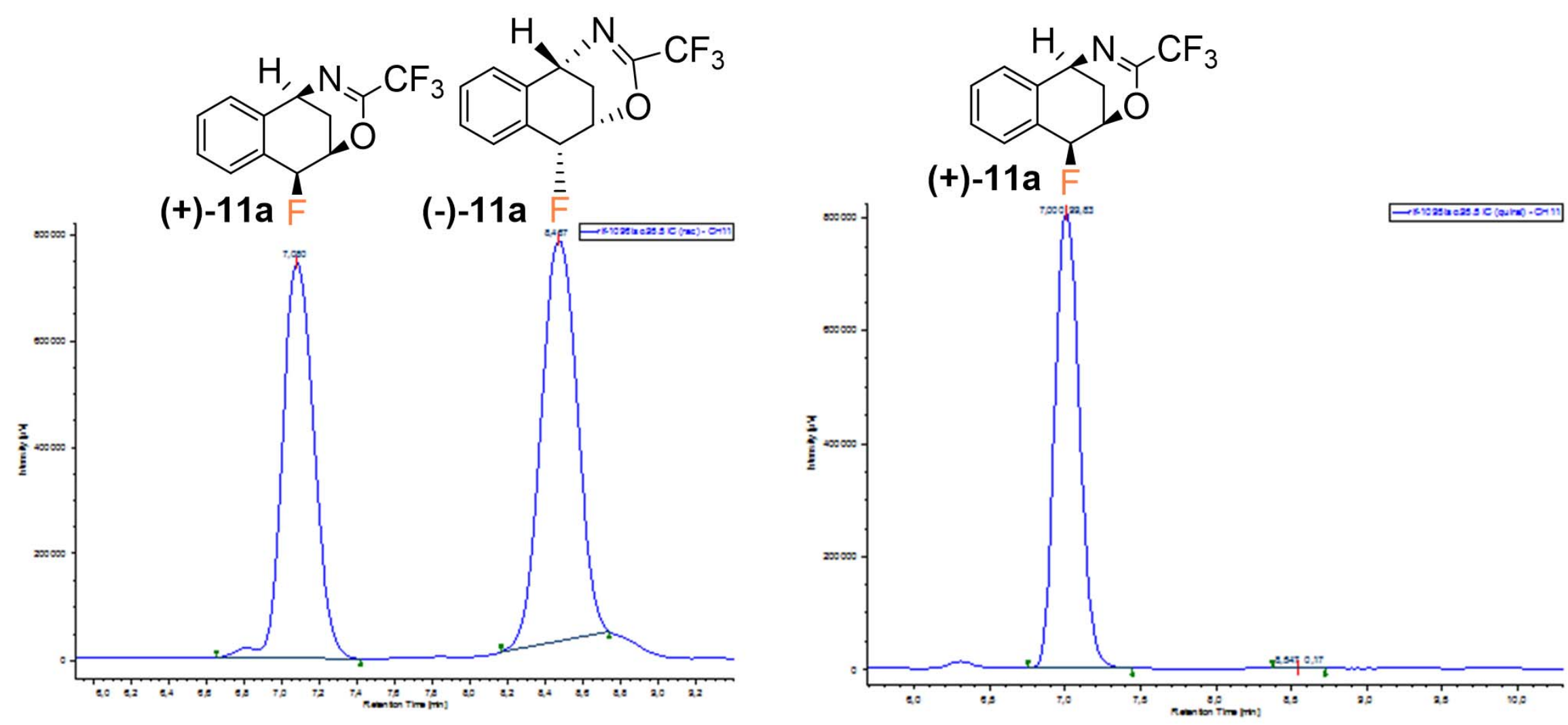
Chiralpak IC, hexane:i-PrOH (99.8:0.2), $1.5 \mathrm{~mL} / \mathrm{min}$
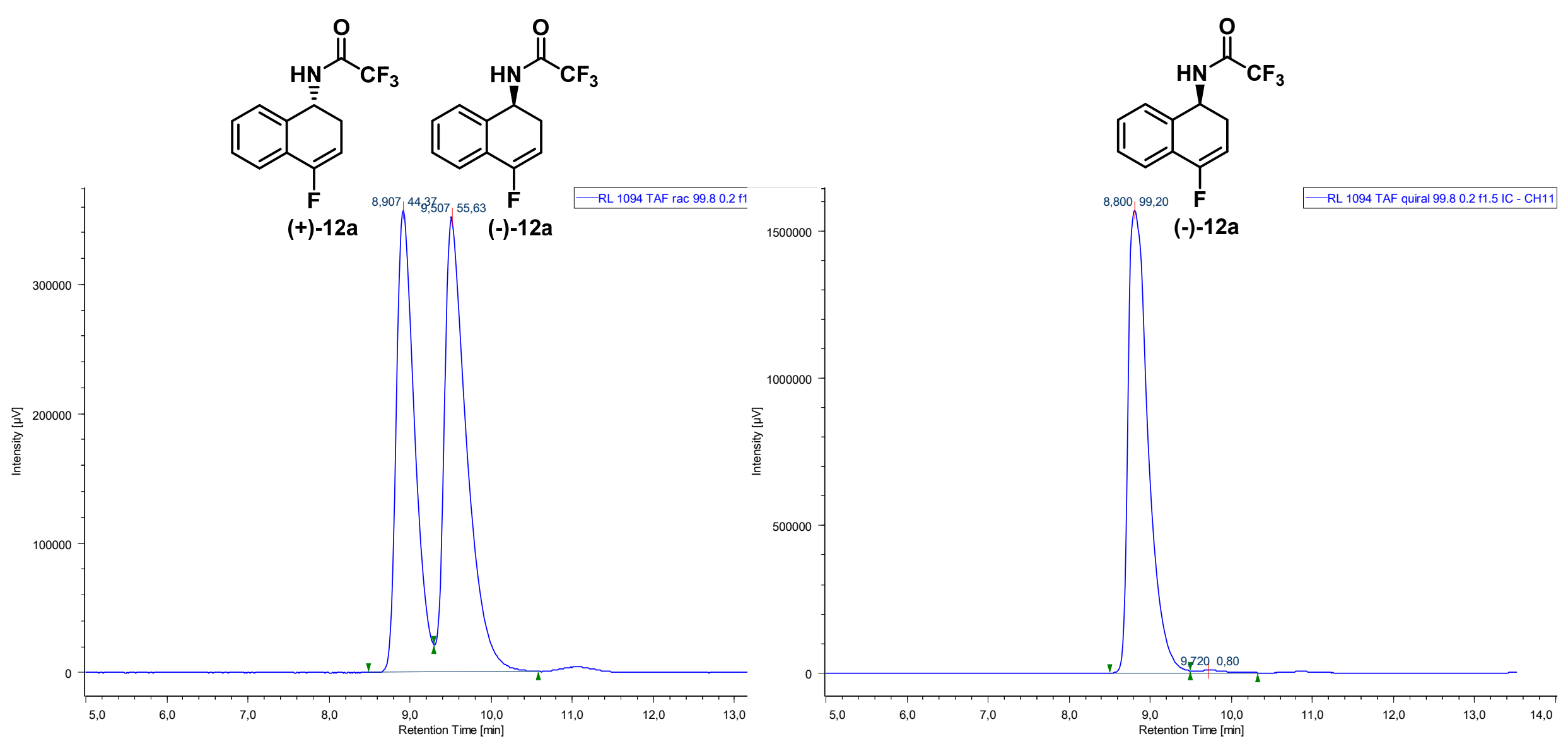
Chiralpak IC, hexane:i-PrOH (99.8:0.2), $1.5 \mathrm{~mL} / \mathrm{min}$

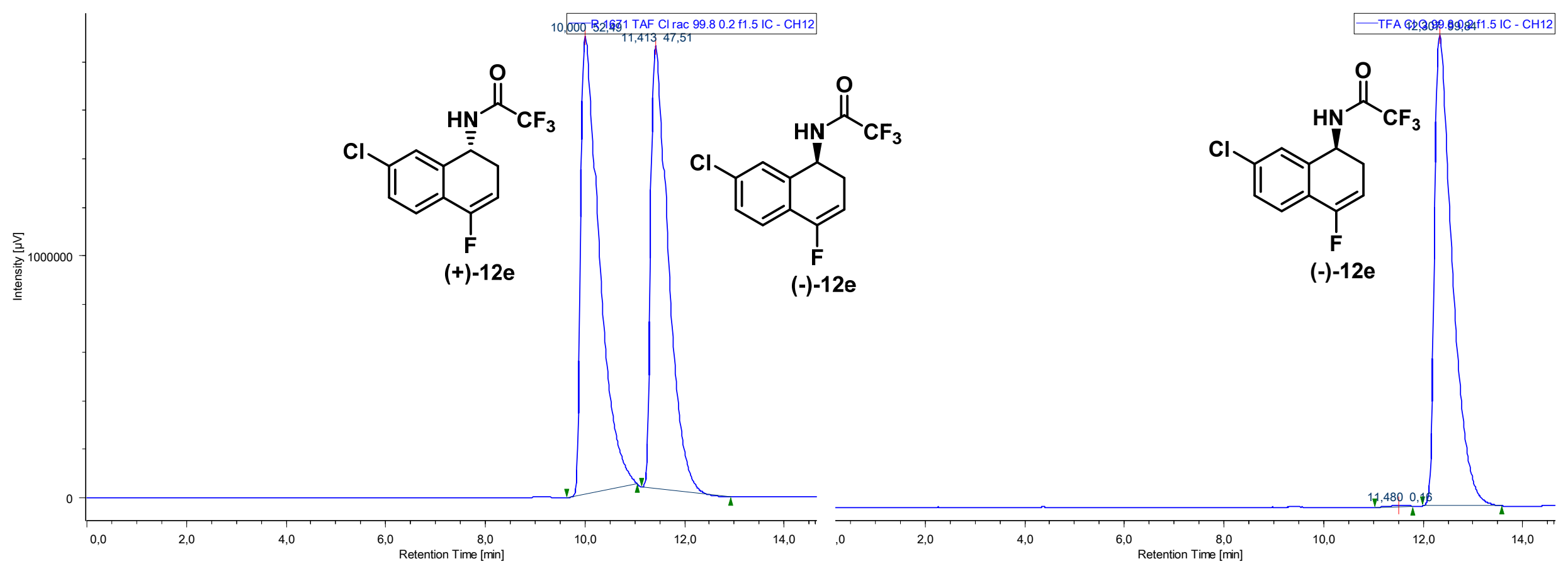




\section{Chiralpak IC, hexane:i-PrOH (99:1), $1 \mathrm{~mL} / \mathrm{min}$}
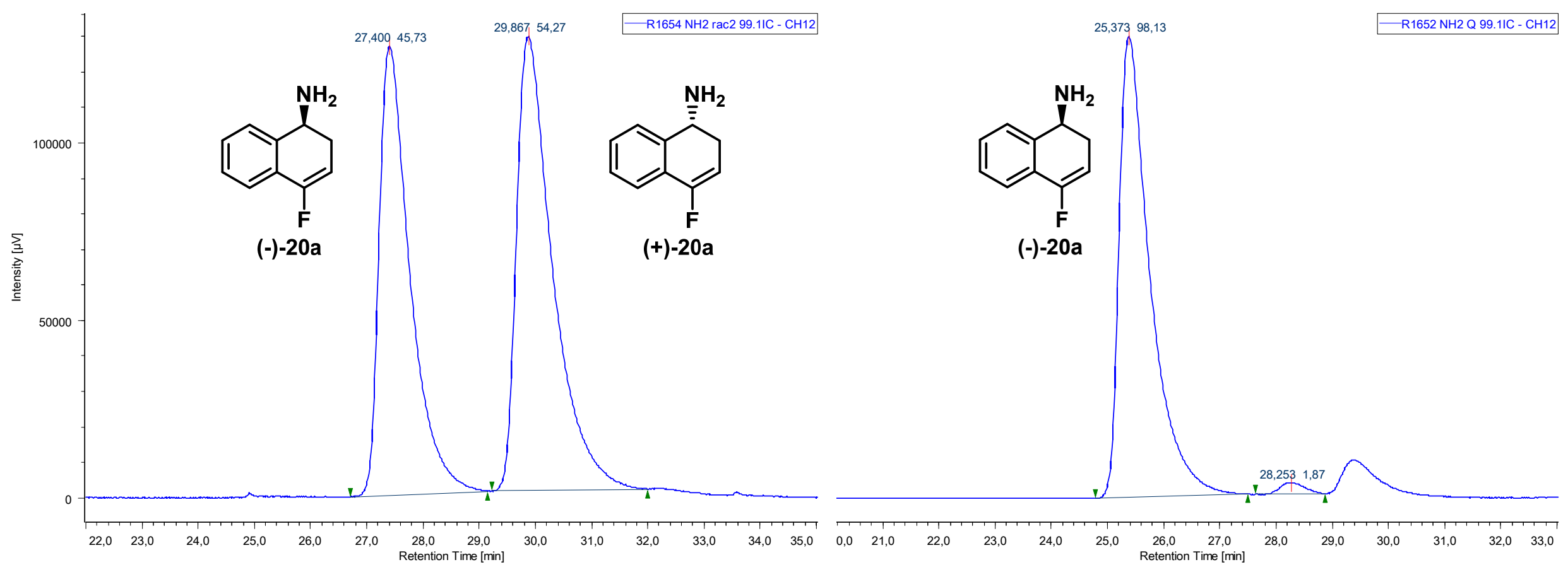


\section{Chiralpak IC, hexane:i-PrOH (99.8:0.2), $1.5 \mathrm{~mL} / \mathrm{min}$}
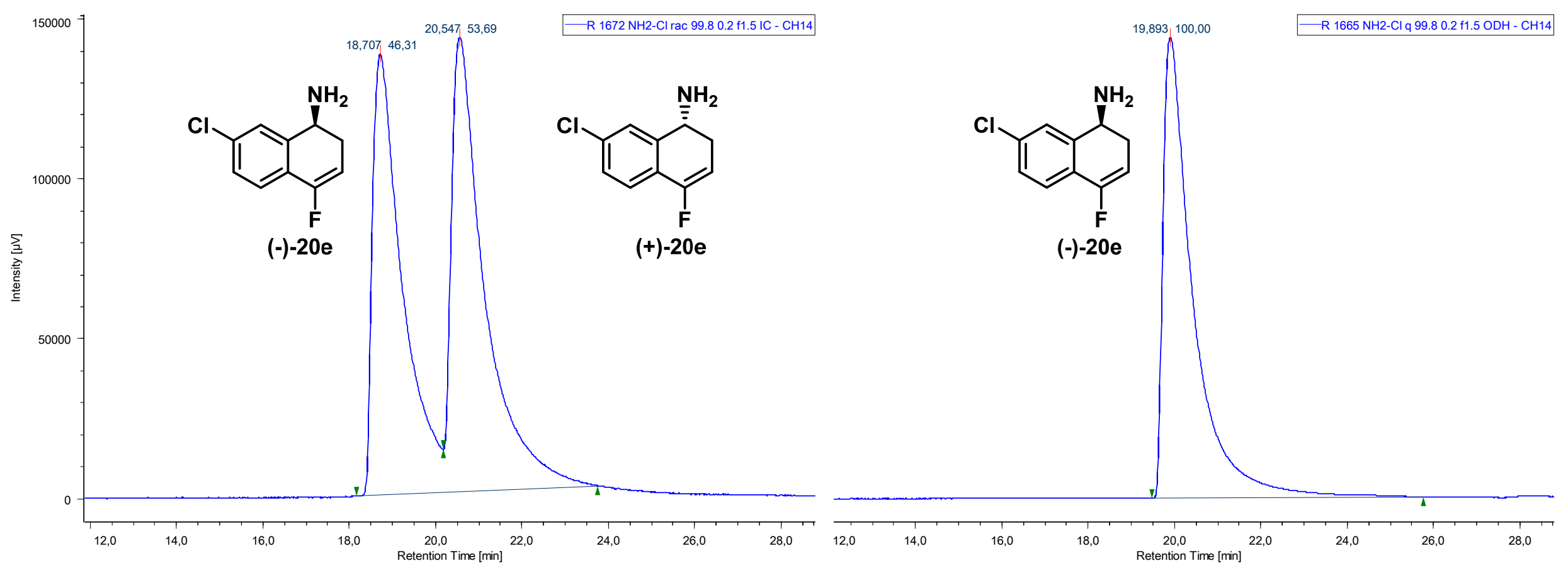